\title{
Annual Report
}

for the

\section{High Energy Physics Program at Texas A\&M University}

\section{DOE Grant DE-FG05-91ER40633}

For the period

November 1, 1991 to October 31, 1992

\author{
Department of Physics \\ Texas A\&M University \\ College Station, Texas 77843
}

November, 1992

\section{DISCLAIMER}

This report was prepared as an account of work sponsored by an agency of the United States Government. Neither the United States Government nor any agency thereof, nor any of their employees, makes any warranty, express or implied, or assumes any legal liability or responsibility for the accuracy, completeness, or usefulness of any information, apparatus, product, or process disclosed, or represents that its use would not infringe privately owned rights. Reference herein to any specific commercial product, process, or service by trade name, trademark, manufacturer, or otherwise does not necessarily constitute or imply its endorsement, recommendation, or favoring by the United States Government or any agency thereof. The views and opinions of authors expressed herein do not necessarily state or reflect those of the United States Government or any agency thereof. 
TABLE OF CONTENTS

Abstract

I. Collider Detector at Fermilab(CDF) Program 1

$\begin{array}{ll}\text { II. The TAMU MACRO Program } & 108\end{array}$

$\begin{array}{ll}\text { III. } & \text { SSC R\&D Program }\end{array}$

IV. Theoretical Physics Program 164 


\begin{abstract}
The Texas A\&M experimental high energy physics program has continued its ambitious experimental activities over the past year. We have continued our participation in two major operating experiments, CDF and MACRO; and have bcen preparing for the next generation of colliding beams experiments through our work with the SDC and GEM programs at the SSC.

In CDF, the Texas A\&M group has lead an effort to design an upgrade for the silicon vertex detector and is currently working with the rest of the collaboration on the our next major data taking run.

In MACRO, we have assisted in the development of the final version of the wave form digitizing system being implemented for the entire scintillator system. This work is nearing completion and we expect to have the system up and running on the detector by summer 1993.

In the area of SSC physics, we have worked within the SDC group to develop gas microstrip chambers for use in precision tracking at the SSC, and in the GEM group our attentions have been directed toward the development of a suitable forward calorimeter design.

The theoretical high energy physics program has also continued to develop during the past funding cycle. D. Nanopoulos and colleagues have continued the study of their very successful string-derived model that unifies all known interactions; flipped SU(5), which is the leading candidate for a TOE. The impact of this work on string phenomenology certainly has far reaching consequences. C. Pope has continued his work on some generalizations of the symmetries of string theory, known as $\mathrm{W}$ algebras. These are expected to have applications in two-dimensional conformal field theory, two-dimensional extensions of gravity and topological gravity and W-string theory.

The following report presents details of the accomplishments of the Texas A\&M program over the past year and the proposed plan of research for the period April 1, 1993 - March 31, 1996.
\end{abstract}




\title{
I. The Fermilab CDF Program
}

The 1992 collider run started in August, three years after the 1988-89 run. Our group focussed its effort on several important areas of programs in the Collider Detector at Fermilab (CDF) experiment. First, we continued contributing to the high level of activity in the physics analyses (QCD, $b$ quark, top quark). In addition, we finished the analysis of the 1991 test beam data of the gas calorimeters for the 1992-93 collider run. We are also actively involved in the SVX-II project for the future collider runs (1995 and beyond).

In the following sections, we will report the impact of these activities and describe our CDF plans for the coming funding cycle.

\section{A. Current Physics Analyses in Progress}

Our group has been heavily involved in several key analysis projects being carried out by the collaboration. During the past year Lou Keeble finished his analysis on the study of four-jet events and the search for double parton (DP) collisions, and submitted this work as his Ph.D. thesis [1]. Teruki Kamon, Peter McIntyre and John Wahl have been developing a new analysis technique to reconstruct $B^{0}$ meson for top quark hunting $[2,3]$. We (Mike Kokkoris, Jeff Wolinski and Teruki Kamon) also began the analysis for $\Lambda_{b}(u d b)$ search using the $d E / d x$ method by the central tracking chamber (CTC) for 1992 data [4]. In the sections which follow, we will briefly cutline the progress being made in each of these areas.

\section{A.1. Study of Four-jet Events}

\author{
Introduction
}

Within the framework of perturbative quantum chromodynamics (QCD), an event having four distinct jets may be produced from a two-body parton interaction through the emission of two high transverse momentum $\left(p_{T}\right)$ gluons. This process is known as double gluon bremsstrahlung; a few of the relevant Feynman diagrams are shown in Figure 1. 
Recently, the full leading order QCD $2 \rightarrow 4$ parton matrix element has been evaluated [5]. This information, combined with proton and anti-proton structure functions, yields predictions for the kinematics and topology of the four-jet double bremsstrahlung process. Since double gluon bremsstrahlung is expected to be the dominant production mechanism for events containing four high $p_{T}$ jets at the Tevatron Collider, we are able to test these predictions at the highest center-of-mass energy currently available.

The kinematics of the four-jet double bremsstrahlung process coupled with a (necessary) minimum jet separation requirement results in the frequent production of jets at small angle with respect to the beam line. We observe that approximately half of the four-jet events collected during the 1988-89 run contain at least one jet in the pseudorapidity range $|\eta|>2.0$. Jet coverage at the CDF is complete in azimuth, and covers the pseudorapidity range $|\eta|<3.5$. This means large acceptance, and an almost complete range of topological freedom for partons in the event sample. A comparison of the kinematic properties of jets between theory and data therefore represents a particularly stringent test of QCD.

In recent years, there has been considerable interest in an alternative method of fourjet production, namely that of the simultaneous scattering of two pairs of partons contained in one $p \bar{p}$ pair [6]. To leading order in the strong coupling parameter $\alpha_{s}$, the double parton scattering process results in the production of two dijet pairs. The interest in this type of event has been partly motivated by the desire to know more about parton correlations within the hadron [7]. Additionally, the production of dijet pairs by double parton scattering is a potential background to interesting Higgs sector physics (i.e. $H^{0} \rightarrow W^{+} W^{-} \rightarrow 4$ jets). Such a background may be severe at future planned hadron colliders such as the SSC or LHC, especially in light of the fact that the occurrence of double parton scattering is expected to increase dramatically with increasing center-of-mass energy $\left(E_{C M}\right)$ [8].

To date, the results of two experimental searches have been published in the literature. The Axial Field Spectrometer (AFS) collaboration finds a significant double parton signal in their data at $\sqrt{s}=63 \mathrm{GeV}$ [9]. However, the more recent analysis of the UA2 group fails to find strong evidence for the process at $\sqrt{s}=630 \mathrm{GeV}[10]$. The center-of-mass energies available to the two experiments were widely different, as were the transverse momenta $\left(p_{T}\right)$ of the jets analysed in each case. This may, in part, be the reason for the different results. The available $E_{C M}$ for $p \bar{p}$ interactions at the CDF is even higher than that available to the UA2 collaboration. This work will therefore complement the work that has already been performed.

In this study, we have found it necessary to examine closely the effects of fifth jets 
in the data, since such effects are not explicitly modelled in the leading order QCD calculations. This has led to a novel method of extracting the double parton content of our data set, involving a cut on the $p_{T}$ of such jets. In addition, we have made particular use of the following two facts: (a) Double parton events exhibit pairwise back-to-back $p_{T}$ balancing; (b) Gluons have a preference for small emission angles.

\section{Detector}

The CDF detector has been described in detail elsewhere [11] and hence only a description of those components relevant to this analysis will be given. In the CDF coordinate system, the $\mathrm{Z}$ axis is taken to lie along the beam line; the polar angle $\theta$ and azimuthal angle $\phi$ are measured with respect to this axis. Additionally, we use pseudorapidity $\eta=-\ln \left(\tan \frac{\theta}{2}\right)$ and we define detector pseudorapidity $\eta_{d}$ as the pseudorapidity measured using the angle $\theta$ subtended at the center of the detector rather than at the interaction point.

Electromagnetic and hadronic calorimeters cover the full range of azirnuthal angle $(\phi)$ in the detector pseudorapidity range $\left|\eta_{d}\right|<4.2$. They are segmented into projective towers pointing towards the center of the detector. The calorimeters occupying the region $|\eta|<1.1$ are scintillator based, and their tower segmentation is $\Delta \eta_{d}=0.1$ and $\Delta \phi=15^{\circ}$. In the region $1.1<\left|\eta_{d}\right|<4.2$ gas calorimeters are used, with $\mathrm{Ar} /$ Ethane (50:50 ratio) as the ionizing medium. Etched copper planes continue the tower geometry with a finer azimuthal segmentation, $\Delta \phi=5^{\circ}$.

Tracking is performed inside an axial 1.4 Tesla magnetic field by the central tracking chamber (CTC) [12]. The event vertex is reconstructed using a vertex time projection chamber (VTPC) that surrounds the beam pipe [13]. 'The vertex position in the $\mathrm{Z}$ direction $\left(\mathrm{Z}_{\text {vert }}\right)$ is observed to be distributed in a Gaussian shape of width $30 \mathrm{~cm}$, centered at $\mathrm{Z}=0 \mathrm{~cm}$. The VTPC is also used to reject events containing second interactions.

\section{Trigger}

The events used in this analysis were collected using a dedicated multijet trigger. The first level (level 0 ) of triggering required a coincidence between the $+Z$ and $-Z$ beam-beam counters, consistent with the timing of a $p \bar{p}$ collision. The next level (level 1) required that the total $E_{T}$ for all trigger towers having $E_{T}>1 \mathrm{GeV}$ be greater than $18 \mathrm{GeV}$. Trigger towers are merged calorimeter towers with the segmentation $\Delta \phi=15^{\circ}, \Delta \eta_{d}=0.2$. The 
level 2 trigger was more sophisticated and performed a crude jet clustering taking trigger towers with $E_{T}>3 \mathrm{GeV}$ and merging them with contiguous towers having $E_{T}>1 \mathrm{GeV}$. The next requirements were then (a) at least 2 clusters $\left(E_{T_{1}}, E_{T_{2}} \geq 3 \mathrm{GeV}\right)$; (b) $\sum E_{T}>80 \mathrm{GeV}$ over the entire calorimeter, including only towers with $E_{T} \geq 1 \mathrm{GeV}$ in either electromagnetic or hadronic components; (c) $\sum E_{T}-E_{T_{1}}-E_{T_{2}}>40 \mathrm{GeV}$ (' $\xi$-cut').

The last requirement functioned as an effective dijet veto, and is labelled as the $\xi$ cut for later reference.

The final level of triggering (level 3) used fully reconstructed calorimeter information. Jets were clustered with the standard CDF algorithm and four jets with $p_{T}>15 \mathrm{GeV} / \mathrm{c}$ were required in the pseudorapidity interval $\left|\eta_{d}\right|<4.2$. At this stage, no correction for jet mismeasurement was performed and the event $Z$ vertex position was assumed to be $Z_{\text {vert }}=0 \mathrm{~cm}$.

\section{Jet Clustering Algorithm}

Jet clustering at CDF is performed using a fixed cone algorithm and has been described in detail elsewhere [14]. The jet cone size $R$ is defined as $R=\sqrt{(\Delta \eta)^{2}+(\Delta \phi)^{2}}$. We used $R=0.7$ in this analysis. Studies have shown that a cone size in the interval between 0.4 and 1.0 includes a major fraction of jet energy and hence is suitable for jet clustering. Note that the level 3 trigger clustering was also performed with a cone size $R=0.7$.

The properties of clustered jets are obtained from electromagnetic $(\mathrm{em})$ and hadronic (had) calorimeters by forming vector sums in the following way,

$$
\begin{aligned}
P_{x} & =\sum_{i}\left(E_{e m}^{i} \sin \theta_{e m}^{i}+E_{h a d}^{i} \sin \theta_{h a d}^{i}\right) \cos \phi^{i} \\
P_{y} & =\sum_{i}\left(E_{e m}^{i} \sin \theta_{e m}^{i}+E_{h a d}^{i} \sin \theta_{h a d}^{i}\right) \sin \phi^{i} \\
P_{z} & =\sum_{i}\left(E_{e m}^{i} \cos \theta_{e m}^{i}+E_{h a d}^{i} \cos \theta_{h a d}^{i}\right), \\
p_{T} & =\sqrt{p_{x}^{2}+p_{y}^{2}} \\
E & =\sum_{i}\left(E_{e m}^{i}+E_{h a d}^{i}\right) \\
E_{T} & =E\left(\frac{p_{T}}{|\vec{P}|}\right) .
\end{aligned}
$$

where the sums are performed over all calorimeter towers passing a $100 \mathrm{MeV}$ minimum $E_{T}$ requirement. Note that this procedure generally produces jets with a non-zero mass. The 
jet position in $\eta$ - $\phi$ space is taken to be that of the cluster $E_{T}$-weighted center-of-mass.

\section{Jet Energy Corrections}

A jet energy correction is performed in order to obtain the most likely parton $p_{T}$ to produce a given jet $p_{T}$ in the calorimeters. The correction is a function of jet $p_{T}$ and $\eta_{d}$ and is performed in two stages.

Relative Correction. There is significant variation in the response of the CDF calorimeters as a function of $\eta_{d}$. A correction for this is applied in the form of a multiplicative factor, dependent on both jet $p_{T}$ and $\eta_{d}$. This factor corrects the $p_{T}$ of a jet anywhere in the calorim $m_{\perp}$ ters to the equivalent $p_{T}$ that would be measured in the region $0.2<\left|\eta_{d}\right|<0.7$ (henceforth referred to as the "central" region). In the central region, jets are primarily measured by the two scintillator based calorimeters, which have the best resolution [15] and whrse energy scales are well determined.

The correction function was constructed using dijet events collected with three single jet triggers. These triggers had level 3 cluster thresholds of $E_{T}>20,40$ and $60 \mathrm{GeV}$. At least one jet was required to be in the central region. For ease of reference, we refer to the jet in the central region as the trigger jet, and the remaining jet as the probe jet. Jets in this sample should, on average, balance back-to-back in $p_{T}$. We interpret any systematic $E_{T}$ imbalance in the calorimeter as indicating an energy scale difference between the central region and the probe jet region. We then form a correction based on the average missing $E_{T}$ $\left(E_{T}\right)$ in these events. We define missing $E_{T}$ as

$$
\vec{E}_{T}=-\sum_{i} E_{T}^{i} \hat{n}_{i}
$$

where $\hat{n}_{i}$ is a unit vector perpendicular to the beam axis and pointing at the $i^{\text {th }}$ calorimeter tower with $|\eta|<3.6$. We then form the missing $E_{T}$ projection fraction $(f)$ as follows,

$$
f=\frac{2 \cdot \overrightarrow{\mathscr{E}}_{T} \cdot{\widehat{p_{T}}}^{\text {probe }}}{p_{T}^{\text {trigger }}+p_{T}^{\text {probe }}}
$$

where $p_{T}^{\text {trig }}$ and $p_{T}^{\text {probe }}$ are the transverse momenta of the trigger and probe jets respectively, and $\widehat{p}_{T}^{\text {probe }}$ is a unit 2 -vector in the transverse plane defined by the direction of the probe jet. The factor of 2 is a result of dividing by the average $p_{T}$ of probe and trigger jets. The missing 
$E_{T}$ is projected along the probe jet axis in order to minimize the effect of any transverse boost due to soft gluon emission. On average then, we have

$$
\vec{E}_{T} \cdot \widehat{p}_{T}^{\text {probe }}=p_{T}^{\text {trigger }}-p_{T}^{\text {probe }}
$$

giving

$$
f=\frac{2 \cdot\left(p_{T}^{\text {trigger }}-p_{T}^{\text {probe }}\right)}{p_{T}^{\text {trigger }}+p_{T}^{\text {probe }}}
$$

Now we can define the relative jet scale as

$$
\beta=\frac{p_{T}^{\text {trigger }}}{p_{T}^{\text {probe }}}
$$

and obtain

$$
\beta=\frac{2+f}{2-f} .
$$

We are thus able to determine the relative jet energy scale $\beta$ as a function of the measured quantity $f$.

In practice, $f$ was determined as a function of $\eta_{d}$ for the $\sum p_{T}$ ranges 50-100, 100-130, $130-170,170-200$ and $>200 \mathrm{GeV} / \mathrm{c}$. A cubic spline fit was then performed to $f$ versus $\eta_{d}$ at 37 positions in $\eta_{d}$, and the $p_{T}$ dependence parameterized linearly. The relative jet energy scale was observed to vary by as much as $30 \%$ before correction, the variation being most pronounced in the cracks between major calorimeter components. As a consistency check, the relative jet correction was applied to the dijet data, and the $f$ variable formed again. The corrected distributions of $f$ were flat as a function of $\eta_{d}$ at the level of a few percent for each of the five ranges of $\sum p_{T}$ (see Figure 2).

Absolute Correction. The absolute jet energy correction is derived using Monte Carlo simulations of the fragmentation process and the CDF detector. The goal is to correct for the effects of detector non-linearity and particle mismeasurement in the central region. Jets falling elsewhere are corrected back to this reference region with the relative jet correction.

Pairs of partons are generated with a flat $p_{T}$ spectrum in the range between 2 and $700 \mathrm{GeV} / \mathrm{c}$, and with a flat $\eta-\phi$ distribution. Fragmentation is performed using a tuned Feynman-Field parameterization $[16,17]$, and the resulting particles are passed to a detailed detector simulation. The response of the central hadron calorimeters has been measured 
both in a test beam and in situ [14]. An underlying event is generated, and the effect of the magnetic field is included in the detector simulation.

Jets are clustered in an identical manner to the data. A matching condition is then applied in order to pair partons with their corresponding cluster. We define $\Delta R_{\text {match }}=$ $\sqrt{\left(\eta_{\text {parton }}-\eta_{\text {cluster }}\right)^{2}+\left(\phi_{\text {parton }}-\phi_{\text {cluster }}\right)^{2}}$ and require it to be less than 0.5 . Cluster $p_{T}$ is then compared to the $\left|\sum \overrightarrow{p_{T}}\right|$ of all particles whose initial direction is contained within the corresponding jet cone. A parameterized fit to the results is shown in Figure 3, where the term corrected $p_{T}$ refers to the particle vector sum $p_{T}\left(\equiv\left|\sum \overrightarrow{p_{T}}\right|\right)$ and clustered jet $p_{T}$ refers to the $p_{T}$ measured with calorimeter information using a cone size of 0.7 . This function defines the absolute jet $p_{T}$ correction. We correct back to the particle level rather than to partons to avoid the theoretical problems involved with the generation of jet mass.

Underlying Event and Cluste-ing Corrections. The term underlying event refers to the radiation of relatively low $p_{T}$ particles due to the fragmentation of spectator partons. These particles often lead to a small upward shift in the measured energy of a jet cluster. In addition, a cluster with significant $p_{T}(>10 \mathrm{GeV} / \mathrm{c})$ will occasionally be produced solely from the underlying event.

Underlying event energy deposition has been studied with data collected with a relatively unbiased trigger, equivalent to just the level 0 requirement of the multijet trigger. For a cone size of 0.7 , an average $E_{T}$ (at the particle level) of approximately $1 \mathrm{GeV}$ is contributed. We make no attempt to correct for this in the four-jet kinematic analysis, since the phenomenon is likely to be process-dependent. We do however use this result to estimate systematic uncertainties due to jet energy scale uncertainty in the double parton analysis.

The nature of the fragmentation process generally results in a certain amount of energy from a parton being lost outside the clustering cone. Using the same Monte Carlo results used in the determination of the absolute jet scale, the particle $\left|\sum \overrightarrow{p_{T}}\right|$ falling outside a cone of 0.7 has been determined. This quantity will be referred to as out-of-cone $p_{T}$ (or $\left.p_{T}^{\text {ooc }}\right)$. The particles are identified as having originated from a parton matching the cluster cone in $\eta$ - $\phi$ space using the criterion of $\Delta R_{\text {match }}<0.5$. The initial particle $\overrightarrow{p_{T}}$ direction is used to decide whether the particle should be classified as inside or outside the cone. Using our fragmentation model, we observe that the out-of-cone $p_{T}$ increases slowly as a function of clustered jet $p_{T}$. We have parameterized the behaviour using the form

$$
p_{T}^{\text {ooc }}=\alpha\left(1-e^{\beta p_{T}}\right)
$$


Using this parameterization, the ratio of $p_{T}^{\text {ooc }}$ to clustered $p_{T}$ as a function of clustered $p_{T}$ has been determined, and is shown in Figure 4. No explicit correction for this effect is made in the four-jet kinematic analysis. Again, the effect is treated as a source of systematic uncertainty on the jet energy scale in the double parton analysis.

\section{Analysis}

Events are selected from the multijet-triggered data sample, and the following data quality cuts are applied: (a) $\left|\mathrm{Z}_{\text {vert }}\right|<60 \mathrm{~cm}$; (b) four (and only four) jets with $p_{T}>$ $25 \mathrm{GeV} / \mathrm{c}$ after correction; (c) jet axis separation $>1.0$ in $\eta$ - $\phi$ plane; (d) $\Sigma p_{T^{\prime}}>140 \mathrm{GeV} / \mathrm{c}$, where the scalar sum of the leading four jets is taken; (e) no second interaction in the VTPC. These cuts will henceforth be referred to as the standard data quality cuts. The cut on the vertex position along the $\mathrm{Z}$ axis, $\left|\mathrm{Z}_{\text {vert }}\right|>60 \mathrm{~cm}$, is necessary to avoid distortion of the projective calorimeter tower geometry. The single jet cut $p_{T}>25 \mathrm{GeV} / \mathrm{c}$ is imposed in order to remove bias introduced by the level 3 trigger. Recall that this trigger passed clusters having $p_{T}>15 \mathrm{GeV} / \mathrm{c}$ uncorrected, assuming a vertex located at $\mathrm{Z}=0 \mathrm{~cm}$. With the subsequent jet correction, and corrections for the true event vertex position, a cluster of $p_{T}=15 \mathrm{GeV} / \mathrm{c}$ may transform into a cluster of $p_{T}=20 \mathrm{GeV} / \mathrm{c}$. This effect was studied in detail using a simulation of the level 3 trigger. The conclusion reached was that $98 \%$ of jets passing a cut of $p_{T}>25 \mathrm{GeV} / \mathrm{c}$ (corrected) would have passed the trigger requirement of $p_{T}>15 \mathrm{GeV} / \mathrm{c}$ (uncorrected). Therefore jets in the sample with $p_{T}>25 \mathrm{GeV} / \mathrm{c}$ are considered unbiased by the level 3 trigger.

The cut on the scalar sum $p_{T}$ of the four jets, $\sum p_{T}>140 \mathrm{GeV} / \mathrm{c}$, removes trigger bias introduced by the level $2 \Sigma E_{T}$ trigger, which required $\Sigma E_{T}>80 \mathrm{GeV}$. This trigger was also simulated in detail, and a comparison made between the level $2 \Sigma E_{T}$ and corrected jet $\Sigma p_{T}$. All simulated events passing an analysis cut of $\Sigma p_{T}>140 \mathrm{GeV} / \mathrm{c}$ were found to also pass the level 2 cut, and hence these events are unbiased. The simulation and data level 2 $\Sigma E_{T}$ are in good agreement, as can be seen in Figure 5.

Due to the large amount of computing power required to run the four-jet and full detector simulation, a fast parton level simulation has been used for much of the analysis in order to obtain reasonably smooth Monte Carlo distributions for comparison with data. This simulation reproduces global jet quantities such as $p_{T}, \eta$ and $\phi$ without the intermediate steps of fragmentation and clustering. As a result, tuning the simulation to agree with existing data is particularly easy. The jet energy scales, both relative and absolute, have simply been 
incorporated in reverse. Also, the jet resclution of the fast simulation has been carefully tuned to agree with dijet data taken with the single jet triggers. For the central region, the resolution is well modeled by the relation [18]

$$
\sigma\left(p_{T}\right)=0.1 \cdot p_{T}+1 \mathrm{GeV} / \mathrm{c}
$$

The resolution in the other regions of calorimeter coverage has been modeled using this simple form, modulated by a multiplicative factor. A quantitative estimate of the uncertainty involved in using the fast jet simulation to model the jet $p_{T}$ resolution has been made. In practice, changing the jet resolution in accordance with this uncertainty was found to have small effect on the distributions produced with the double bremsstrahlung simulation, and was a minor source of systematic error in the $\therefore$ uble parton analysis.

\section{QCD Comparison}

The large number of Feynman diagrams which arise in the calculation of the full double bremsstrahlung matrix element result in a very time-consuming computer simulation. Consequently, considerable effort has been invested into formulating fast and accurate approximations. This analysis has primarily made use of the approximation of Kunszt and Stirling [19], though a different approximation (Maxwell [20]) has also been used in order to partially estimate systematic uncertainties arising from not using the exact matrix element expression. Specifically, this analysis has used the computer routine PAPAGENO [21] to perform all QCD simulations.

In order to avoid the singularities inherent in the matrix element calculation and increase generation efficiency, the following cuts are placed on partons generated with the double bremsstrahlung simulation: (a) $p_{T}>13 \mathrm{GeV} / \mathrm{c}$; (b) separation $|\Delta R|>0.8$; (c) $\left|\eta_{d}\right|<4.0$.

When the standard data quality cuts are applied to the jets resulting from these partons, the chosen generation levels are $100 \%$ efficient. Shown in Figure 6 are distributions of parton $p_{T}$, separation and $\eta_{d}$ after applying the standard cuts. Also shown for comparison are the $\eta_{d}$ distributions for the smallest and largest $p_{T}$ jets in the data. The distribution of $\eta_{d}$ for jets in the data is slightly depleted at $\left|\eta_{d}\right| \sim 1.1$ and $\left|\eta_{d}\right| \sim 2.2$. This is due to the level $2 \xi$ cut, which preferentially removed events containing jets in these regions. With the standard data quality cuts in place, this cut is approximately $85 \%$ efficient. 
Parton distributions have also been generated using a uniform matrix element (phase space) simulation in place of the QCD four-jet approximation. Comparing results from both matrix elements will indicate those characteristics which are specific to QCD as opposed to being a consequence of four-parton kinematics. Again, the fast detector simulation was used to transform partons into jets, and the standard analysis cuts were applied.

A comparison of the $p_{T}$ spectra between data and QCD for each of the four jets has been performed. Before comparison, the jets are ordered in $p_{T}$, jet 1 has the largest $p_{T}$ after correction, jet 2 the next largest and so on. Also, we have formed the scalar sum $p_{T}$ of all four jets. 'The results are shown in Figures 7 and 8 . The phase space predictions are not shown here because these distributions are governed mainly by kinematics; there is little difference between the results obtained with the QCD four-jet matrix element and the uniform matrix element. However, there is no significant deviation between the data and the QCD predictions.

In order to describe the topology of the four-jet process, nine variables in total are nreded. Three of these are used to boost the system to the center-of-mass reference frame.

fortunately, with so many remaining degrees of freedom, it is not possible to construct a global Dalitz-type plot as can be done in a three-jet analysis. Consequently, we have chosen to use angles between jets, in keeping with the analysis of the UA2 collaboration. In the center-of-mass system we define the angle between jets $i$ and $j$ as $\Omega_{i j}$ and use the variables $\cos \Omega_{i j}$ for our comparison. The results for the data, together with the QCD fourjet prediction and the phase space results are shown in Figure 9. The distributions have been normalized to unit area.

In all six cases, the observed agreement between the data and QCD is impressive. This time, the angular distributions obtained with a phase space generator are quite different to the QCD results, and tend to peak at $\cos \Omega_{i j} \sim 0$. This corresponds to the configuration where parton pairs are $90^{\circ}$ apart in azimuth. The QCD matrix element does not exhibit this peaking, preferring instead configurations where partons are close together. Note that the four-jet double bremsstrahlung calculation is evaluated only to leading order in the strong coupling parameter $\alpha_{s}$. The agreement between data and theory suggests that higher order terms in the perturbation series have little effect on the jet topology.

\section{Double Parton Analysis}

The rate of double parton scattering depends strongly on the number of partons in 
each colliding hadron, $N\left(x, Q^{2}\right)$. In the simplest approximation, $\sigma_{\mathrm{DP}} \propto N^{2}$, where $\sigma_{\mathrm{DP}}$ is the double parton scattering cross section. The corresponding dependence for the double bremsstrahlung cross section $\left(\sigma_{\mathrm{DB}}\right)$ is linear, $\sigma_{\mathrm{DB}} \propto N$. For large enough $N$, double parton scattering will therefore dominate. At constant momentum transfer $Q^{2}$, parton d sities increase with decreasing Feynman $x$ [22]. Therefore experiments operating at hic, "r center-of-mass energies will produce a higher rate of double parton events relative to double bremsstrahlung events for a given minimum jet $p_{T}$ requirement. However, the relative rate is expected to decrease as this minimum $p_{T}$ is increased; in one approximation [23]

$$
\mathcal{R}=\frac{N_{\mathrm{DP}}}{N_{\mathrm{DB}}} \propto \frac{1}{p_{T}^{2}}
$$

Qualitatively then, the high CDF center-of-mass energy is an advantage in searching for double parton events, whereas the fairly high cut on transverse momentum, $p_{T}>$ $25 \mathrm{GeV} / \mathrm{c}$, is somewhat restrictive. In order to obtain a more quantitative estimate of the relative double parton to double bremsstrahlung rate, we use the following simple factorized form:

$$
\sigma_{\mathrm{DP}}=\frac{\sigma_{\mathrm{dijet}}^{2}}{2 \sigma_{\mathrm{eff}}},
$$

where $\sigma_{\text {dijet }}$ is the dijet cross section, and $\sigma_{\text {eff }}$ is the unknown effective cross section. This equation assumes that parton correlations may be neglected; such a condition is expected to apply for center-of-mass energies up to $40 \mathrm{TeV}[24,25]$. The factor of 2 is used for ease of comparison between this work and earlier results.

The physical significance of $\sigma_{\text {eff }}$ can be seen by considering a single $p \bar{p}$ interaction of integrated luminosity $\int \mathcal{L} d t$. The number of double parton interactions is given by

$$
N_{\mathrm{DP}} \quad \int \mathcal{L} d t \sigma_{\mathrm{DP}}=\left(\int \mathcal{L} d t \sigma_{\text {dijet }}\right)\left[\left(\frac{1}{2 \sigma_{\text {eff }}}\right) \sigma_{\text {dijet }}\right] .
$$

The first term on the right hand side of the above equation represents the number of dijet events, and the second term corresponds to the number of additional dijet interactions. Therefore we may associate the term $1 / 2 \sigma_{\text {eff }}$ with an effective luminosity. Now, in producing the original dijet interaction, a $p \bar{p}$ collision must have occurred. If we assume that the partonic remnants interact inelastically, then $2 \sigma_{\text {eff }}$ can be associated naively with the total inelastic cross section of approximately $40 \mathrm{mb}$. However, since the first interaction preferentially selects small impact parameters, the effective luminosity is increased by approximately a factor of $2.3[26]$. This suggests that a value $\sigma_{\mathrm{eff}}=8.7 \mathrm{mb}$ is reasonable for a preliminary estimate of the double parton cross section. 
Assuming a value for $\sigma_{\text {efi }}$ of $8.7 \mathrm{mb}$, we have determined the relative parton level cross sections for both double parton and double bremsstrahlung processes. The double parton simulation was constructed by merging two dijet events at the parton level in order to produce the characteristic topology. Each dijet event was independently given a small transverse kick in order to simulate the effect off additional gluon radiation. Our model assumes no parton correlations. The results are plotted in Figures 10a and 10b as functions of both minimum jet $p_{T}$, and jet scalas $\Sigma p_{T}$ respectively. These figures indicate that the double parton signal will be small; note however that the uncertainty on the absolute value of $\sigma_{\mathrm{DB}}$ is approximately a factor of two.

The following variables have been used in our search for double parton events:

(a) $S$, or the 'Significance', defined as

$$
S(1+2,3+4) \equiv \sqrt{\left[\left(\frac{\left|p_{T_{1}}+p_{\mathrm{T}_{2}}\right|}{\sqrt{p_{T_{1}}+p_{T_{2}}}}\right)^{2}+\left(\frac{\left|\vec{p}_{T_{3}}+p_{\mathrm{T}_{4}}\right|}{\sqrt{p_{T_{3}}+p_{T_{4}}}}\right)^{2}\right] / 2}
$$

$S$ is minimized over the three possible independent jet pairings. This variable exploits the $p_{T}$-balancing of double parton dijet pairs.

(b) $\Delta_{S}$; after $S$ minimization, $\Delta_{S}$ is the azimuthal angle between the resultant dijet $\Sigma \overrightarrow{p_{T}}$ for the two matched jet pairs. This variable takes advantage of differences in angular correlations between the double parton and double bremsstrahlung processes.

Using simulations of both the double parton and double bremsstrahlung processes, a quantitative analysis of the respective signal-finding ability of the $S$ and $\Delta_{S}$ variables has been performed. The parton level events are passed to the fast detector simulation so that the effects of jet mismeasurement and resolution smearing are included. An artificial sample is then constructed, comprised of $10 \%$ signal (double parton) and $90 \%$ background (double bremsstrahlung) events. The variables $S$ and $\Delta_{S}$ are shown in Figure 11 for signal and background. The artificial sample is then fitted to a normalized admixture of signal and background shapes and the $\chi^{2}$ per degree of freedom $\left(\chi_{r}^{2}\right)$ using Poissonian statistics is evaluated. The dependence of the $\chi_{r}^{2}$ as a function of double parton content is interpreted as a measure of the signal finding resolution of each variable. The results of this test are shown in Figure 12. The most effective variable is $\Delta_{S}$, followed closely by $S$. Note that the $\Delta_{S}$ variable has the desirable property of being peaked for double parton events in a local minimum :or double bremsstrahlung events. 
Using the same fitting technique, the four-jet data has also been fitted to an admixture of simulated double parton and double bremsstrahlung distributions, for both variables. The only free parameter used is the relative fraction of signal to background, $(\mathcal{R})$. The results are shown in Figures 1ila and $13 \mathrm{~b}$. While each of the fits look reasonable $\left(\chi_{r}^{2} \sim 1.2\right)$ there is a marked difference beiween the value of $\mathcal{R}$ found using the $S$ variable $(\sim 2 \%)$, and those found with $\Delta_{S}(\sim 16 \%)$.

A reasonable explanation for this difference can be provided by considering the effect of additional clusters in the data, either from the underlying event or from gluon radiation. Such clusters will tend to destroy the $p_{T}$-balancing in a four-jet event. Also, the angular predictions of the exclusive four-jet Monte Carlo program for the double bremsstrahlung process may not strictly apply; the distribution of $\Delta_{S}$ may be distorted by these extra clusters. This hypothesis has been tested using a Monte Carlo simulation of five-jet events, where four (and only four) jets are required to pass the standard analysis cuts. Five-jet events were generated according to the (dominant) gluon scattering matrix element $g g \rightarrow g g g g g$. This approximation is adequate in order to estimate the topological effects of an additional jet. The distributions obtained are shown in Figure 14. The effect of a fifth jet is to reduce the double parton signal region relative to the exclusive four-jet prediction for $S$, and enhance the signal for $\Delta_{S}$.

The effect on the topological variables due to clusters produced by the underling event was checked using a phenomenological simulation of the underlying event in conjunction with the four-jet and full detector simulations [1]. We observe that very few underlying event clusters with $p_{T}>25 \mathrm{GeV} / \mathrm{c}$ are produced. Therefore we conclude that the difference in $\mathcal{R}$ between $p_{T}$-balancing and angular variables is not primarily due to underlying event clusters. Rather, it is due to the effect of fifth clusters in the data produced in general by QCD gluon radiation from the hard scattering process.

As a further test of this conclusion, we have performed a cut on the $p_{T}$ of fifth jets $\left(p_{T_{5}}\right)$ in our data sample. The effect of the cut $p_{T_{5}}<15 \mathrm{GeV} / \mathrm{c}$ on the shapes of $S$ and $\Delta_{S}$ can be seen in Figures 15 and 16, respectively. As expected, the exclusion of events containing a high $p_{T}$ fifth jet leads to an increase in the double parton signal found using the $S$ variable, and a decrease in that found using $\Delta_{S}$. The trend towards convergence in the fitted value of $\mathcal{R}$ found using these two different variables can be seen clearly in Figure 17.

The value of $\mathcal{R}$ used in this analysis is determined in two stages. We first fit $S$ and $\Delta_{S}$ versus the $p_{T_{5}}$ cut to separate straight lines, as shown in Figure 17. The point of intersection of these lines determines $\mathcal{R}$; the value of $p_{T_{5}}$ at this point we label $p_{T_{5}^{\prime}}$. Making the 
cut $p_{T_{5}}<p_{T_{5}^{\prime}}$, we then perform a combined fit for $S$ and $\Delta_{S}$ to an admixture of signal and background shapes. The statistical uncertainty on $\mathcal{R}$ is then taken to be the change in $\mathcal{R}$ necessary to increase $\chi_{r}^{2}$ by 1 unit. This method is chosen since it takes into account the effect of correlations between the two variables. The result obtained using structure function EHLQ1 with $Q=\left\langle p_{T}\right\rangle$ in both double bremsstrahlung and double parton simulations is $\mathcal{R}=0.053 \pm 0.013$ (statistical error only).

\section{Double Interactions}

As a result of the high luminosity conditions $\left(\mathcal{L} \approx 5 \times 10^{-29} \mathrm{~cm}^{-2} \mathrm{sec}^{-1}\right)$ of the 1988-89 run, about $20 \%$ of events taken with the multijet trigger contain two separate $p \bar{p}$ interactions. If both interactions produce dijet events, then the event topology will be indistinguishable from that of the double parton process. Fortunately, we are able to reject around $85 \%$ of these events using the VTPC. The remaining $15 \%$ cannot be rejected because the two interactions occurred close together, and cannot be resolved by the VTPC. After the cut on secondary vertices, around $3 \%$ of events have an unresolved secondary intersction. Fortunately, the vast majority of events containing two interactions do not produce dijet pairs. In fact, the cross section for the production of one double bremsstrahlung event, and one soft ("minimum bias") event is far greater. This has been tested by constructing the $S$ distribution for events containing two interactions. The shape is entirely consistent with that of the rest of the four-jet data, comprised mainly of double bremsstrahlung events. Therefore, double interactions are neglected as a source of background to the double parton process.

\section{Limits}

Although the double parton signal in the four-jet data is statistically significant at the $4 \sigma$ level, systematic uncertainties substantially decrease this significance. We therefore place limits on the double parton scattering cross section $\sigma_{\mathrm{DP}}$, and on the effective cross section $\sigma_{\mathrm{eff}}$.

Limit on $\sigma_{\mathrm{DP}}$. The double parton cross section can be expressed as

$$
\sigma_{\mathrm{DP}}=\frac{N_{\mathrm{DP}}}{\int \mathcal{L} d t \cdot \mathcal{A}_{\mathrm{DP}}^{\text {cuts }} \cdot \mathcal{A}_{\mathrm{DP}}^{\text {trig }}}
$$

where $N_{\mathrm{DP}}$ is the number of events due to double parton scattering in the data, $\int \mathcal{L} d t$ is the integrated luminosity of the event sample, $\mathcal{A}_{\mathrm{DP}}^{\text {cuts }}$ is the acceptance of the four-jet event cuts, and $\mathcal{A}_{\mathrm{DP}}^{\text {trig }}$ is the acceptance of the multijet trigger for double parton events. 
Double parton events were generated with parton $p_{T}>18 \mathrm{GeV} / \mathrm{c}$. No partons below this $p_{T}$ survive the standard analysis cut $p_{T}$ (corrected) $>25 \mathrm{GeV} / \mathrm{c}$ Therefore our limit on $\sigma_{\mathrm{DP}}$ will refer to to the cross section for partons with $p_{T}>18 \mathrm{GeV} / \mathrm{c}$.

The luminosity of the event sample has been determined to be

$$
\int \mathcal{L} d t=325 \mathrm{nb}^{-1} \pm 7 \%
$$

Details of the CDF luminosity determination and its associated uncertainty can be found elsewhere [27]. The acceptance of event cuts for double parton events ( $\left.\mathcal{A}_{\mathrm{DF}}^{\text {cuis }}\right)$ has been determined using the double parton simulation, in conjunction with the fast jet Monte Carlo program. We find

$$
\mathcal{A}_{\mathrm{DP}}^{\text {cuts }}=(6.49 \pm 0.92) \times 10^{-3}\left(\text { parton } p_{T}>18 \mathrm{GeV} / \mathrm{c}\right)
$$

This acceptance is small since the analysis cuts only become fully efficient for parton $p_{T} \sim$ $30 \mathrm{GeV} / \mathrm{c}$, and we include all partons with $p_{T}>18 \mathrm{GeV} / \mathrm{c}$. The main systematic uncertainty on $\mathcal{A}_{\mathrm{DP}}^{\text {cuts }}$ stems from variations in existing structure functions and allowed $Q^{2}$ parameterizations. Note that this analysis is particularly sensitive to structure function uncertainty since it involves partons with low Feynman $x$.

A wide range of structure functions were used in the evaluation of this uncertainty, including Morfin-Tung DIS [28], MRS1, 2 and 3 and Martinelli sets 1, 2 and 3. We use $Q=\left\langle p_{T}\right\rangle$ and $Q=\left\langle p_{T} / 2\right\rangle$ to estimate the uncertainty due to the $Q^{2}$ parameterization. The quoted acceptance and uncertainty represent the average and standard deviation of results calculated with all the above mentioned structure functions. In addition, a very small systematic uncertainty (around $2 \%$ ) arises from uncertainty in the modeling of jet resolution by the fast jet simulation.

The acceptance of the trigger for double parton events has been determined using a sample of double parton events with full detector and trigger simulations. The biggest loss in acceptance is due to the level 2 cut on $\xi$ (defined earlier); the analysis cuts ensure almost complete acceptance for the level 2 cut on $\Sigma E_{T}>140 \mathrm{GeV}$ and the level 3 cut on $p_{T}($ jet $)>25 \mathrm{GeV} / \mathrm{c}$. We find

$$
\mathcal{A}_{\mathrm{DP}}^{\text {trig }}=0.85 \pm 0.10
$$

Including uncertainties linked with choice of structure function, $Q^{2}$ parameterization and jet resolution uncertainty we find

$$
\mathcal{R}=0.053 \pm 0.013 \text { (stat.) } \pm 0.003 \text { (syst.). }
$$


The uncertainty in structure function and $Q^{2}$ parameterization was estimated by refitting the data with simulations generated with different combinations of structure functions EHLQ1 and DO2 with $Q=\left\langle p_{T}\right\rangle$ and $Q=\left\langle p_{T} / 2\right\rangle$. Note that the $\xi$-cut at level 2 was also studied in detail in order to examine its effect on the two topological variables, $S^{\prime}$ and $\Delta_{S}$. This trigger cut was not found to bias either of these variables, and hence was not a factor in the determination of systematic errors on $\mathcal{R}$.

We assume the total number of four-jet everits in our data sample as $N_{\text {tot }}=N_{\mathrm{DB}}+N_{\mathrm{DP}}$. This gives the result

$$
N_{\mathrm{DP}}=\mathcal{R} \cdot \frac{N_{\text {tot }}}{1+\mathcal{R}}=0.0529 \cdot \frac{2213}{1+0.0529}=111
$$

The largest uncertainty on $\sigma_{\mathrm{DP}}$ is associated with the jet energy scale. There is some cancellation of this uncertainty since it effects both $N_{\mathrm{DP}}$ and $\mathcal{A}_{\mathrm{DP}}^{\text {cuts }}$ in the same direction. The following changes were made to the jet scale in order to evaluate the effect on $\sigma_{\mathrm{DP}}$ : (a) the absolute jet energy scale was raised and lowered by $5 \%$; (b) the relative jet energy scale was increased and decreased by an amount reflecting the statistical uncertainty inherent in its determination. The region $0.2<|\eta|<0.7$ was not altered; (c) a correction was performed for underlying event energy inside the clustering cone; (d) a correction was performed for energy lost outside the clustering cone.

The resulting change in the ratio $N_{\mathrm{DP}} / \mathcal{A}_{\mathrm{DP}}^{\text {cuts }}$ is found to be $+20 \%$ and $-27 \%$. Inserting all determined quantities and combining systematic uncertainties in quadrature we obtain the result,

$$
\sigma_{\mathrm{DP}}=65+25-28 \mathrm{nb}
$$

The inclusion of systematic errors has therefore reduced the significance of this result to a $2 \sigma$ effect. The upper limit on $\sigma_{\mathrm{DP}}$, assuming a Gaussian distribution of uncertainties, is

$$
\sigma_{\mathrm{DP}}<0.11 \mu \mathrm{b} \quad\left(\text { parton } p_{T}>18 \mathrm{GeV} / \mathrm{c}\right)
$$

Limit on $\sigma_{\text {eff. }}$. Using the result for $\sigma_{\mathrm{DP}}$, the effective cross section, $\sigma_{\text {eff }}$ can easily be calculated, and a lower limit may be established on this quantity. The only additional piece of information required is the dijet cross section, $\sigma_{\text {dijet }}$. We have used a theoretical evaluation calculated with the PAPAGENO program,

$$
\sigma_{\text {dijet }}=39 \mu \mathrm{b} \pm 20 \%,
$$


Table 1: A summary of the results, experimental parameters and event cuts for the double parton analyses performed by the AFS, UA2 and CDF collaborations.

\begin{tabular}{cccccc}
\hline & $\sqrt{s}(\mathrm{GeV})$ & $p_{T}^{\min }(\mathrm{GeV} / \mathrm{c})$ & $\eta$ Range & $N_{\text {events }}$ & Result \\
\hline AFS & 63 & 4 & $|\eta|<1$ & $\approx 1,000$ & $\sigma_{\text {eff }}=5 \mathrm{mb}$ \\
UA2 & 630 & 15 & $|\eta|<2$ & $\approx 10,000$ & $\sigma_{\text {eff }}>8.3 \mathrm{mb}(95 \%$ C.L. $)$ \\
CDF & 1800 & 25 & $|\eta|<3.5$ & $\approx 2,000$ & $\sigma_{\text {eff }}>4.2 \mathrm{mb}(95 \%$ C.L. $)$ \\
\hline \hline
\end{tabular}

for partons with $p_{T}>18 \mathrm{GeV} / \mathrm{c}$. The uncertainty was estimated by taking various structure functions and $Q^{2}$-parameterizations used in the calculation of $\mathcal{A}_{\mathrm{DP}}^{\text {cuts }}$.

The final result for $\sigma_{\text {eff }}$ is,

$$
\sigma_{\mathrm{eff}}=11.7+6.3-5.9 \mathrm{mb}
$$

where all uncertainties have been added in quadrature. The limit on $\sigma_{\text {eff }}$ is calculated at the $9.5 \%$ confidence level to be,

$$
\sigma_{\text {eff }}>4.2 \mathrm{mb}(95 \% \text { C.L. }) \text {. }
$$

and at the $90 \%$ confidence level we obtain,

$$
\sigma_{\text {eff }}>5.6 \mathrm{mb}(90 \% \text { C.L. }) \text {. }
$$

This result therefore is consistent with the UA2 limit of $\sigma_{\text {eff }}>8.3 \mathrm{mb}$ (95\% C.L.).

A summary of the experimental parameters and final results of previous searches for double parton scattering compared to those of CDF is shown in Table 1. It should be noted that there are large variations in center-of-mass energies and minimum $p_{T}$ cut-offs. A comparison between these results can be made if one assumes $\sigma_{\text {eff }}$ is constant over the wide range of existing experimental conditions.

\section{Implications for Physics at $40 \mathrm{TeV}$}

In order to investigate the implications of the CDF result for physics at the SSC, parton level events have been generated using $E_{C M}=40 \mathrm{TeV}$ and $\sigma_{\text {eff }}=11.7 \mathrm{mb}$. The cross sections for simulated double parton and double bremsstrahlung events as a function of the $p_{T}$ of the softest jet $\left(p_{T_{4}}\right)$ is shown in Figure 18. Events have been generated with 
$p_{T}>10 \mathrm{GeV} / \mathrm{c}$. Based on this study, one would expect to see a significant double parton signal at the SSC for jets with a minimum $p_{T}$ cut below $60 \mathrm{GeV} / \mathrm{c}$. In fact, the double parton mechanism apparently dominates in the range $p_{T_{4}}<40 \mathrm{GeV} / \mathrm{c}$. It should be noted that there is a large uncertainty on $\sigma_{\mathrm{eff}}$; the value of $11.7 \mathrm{mb}$ is the best-fit value found using CDF data. Also, the uncertainty due to the choice of structure function used in theoretical evaluation of $\sigma_{\mathrm{DB}}$ is expected to be large.

\section{Conclusions}

We have studied the production of four-jet final states in $p \bar{p}$ collisions at $\sqrt{s}=1.8 \mathrm{TeV}$. We find that the leading order QCD prediction for the topology of the double bremsstrahlung process is consistent with CDF four-jet data above a corrected $p_{T}$ of $25 \mathrm{GeV} / \mathrm{c}$. While a small double parton signal is required to best fit the data, the significance of this signal is small when both statistical and systematic uncertainties are included. As a result, limits have been established on both $\sigma_{\mathrm{DP}}$ and $\sigma_{\text {eff }}$ employing a technique which uses a combination of $p_{T}$-balancing and angular variables. The existence of small additional clusters due primarily to gluon radiation was observed to be an important effect in setting these limits.

Using the best fit value of $\sigma_{\text {eff }}=11.7 \mathrm{mb}$, a Monte Carlo study has indicated that double parton scattering may be the dominant production mechanism for events containing four jets where the smallest jet satisfies the requirement $p_{T}<40 \mathrm{GeV} / \mathrm{c}$ at a center-of-mass energy of $40 \mathrm{TeV}$. This underscores the importance of the double parton scattering process as a background to any process leading to the production of two pairs of jets, balanced backto-back in $p_{T}$. We hope that the methods developed in this analysis will be immediately applicable to four-jet physics at the SSC when these colliders become operational.

\section{A.2. Top Quark Search Through Partial $B$ Reconstruction}

\section{Introduction}

We have been developing a new physics analysis to increase the sensitivity of the search for the top quark in CDF. The physics analysis would identify a $b$ quark. This is achieved by a partial reconstruction of the subsequent characteristic decay of the $b$ quark [2]. 
The result would provide a measurement of the mass of each top quark candidate, hence a peak in the mass spectrum for a sample of event candidates.

\section{Top Quark}

Combining the data from the LEP, UA1, UA2 and CDF experiments, the mass of the top is predicted to be $137 \pm 40 \mathrm{GeV} / \mathrm{c}^{2}$, assuming the Standard Model is correct [29]. This is the best prediction for the mass of the top quark from indirect measurements. Direct discovery of the top quark is only possible at the Fermilab Tevatron in the 1990's.

The top quark can be pair-produced ( $t \bar{t}$ pairs) in $1.8 \mathrm{TeV}$ proton-antiproton collisions. Figure 19 shows the cross-section for top quark production at $\sqrt{s}=1.8 \mathrm{TeV}$, as a function of top quark mass. If the top quark mass is $120 \mathrm{GeV} / \mathrm{c}^{2}$, it will be produced 3000 times during 1992-93 run.

\section{Bottom Quark Identification Technique}

The challenge to the experimentalist is to find the rare events where a top quark is produced in a $\vec{p} p$ collision and decays with a characteristic signature. Each top quark of a pair would decay into a $W$ and a $b$ quark. The weak boson in turn could be readily recognized through its $\beta$ decay into a lepton $\ell$ (electron $e$ or muon $\mu$ ) and neutrino $\nu_{\ell}$. Such decays are observed as an isolated high-energy lepton and an apparent missing transverse energy, $\not_{T}$ from the undetected neutrino.

In collisions producing a $t \bar{t}$ pair, one possible final state consists of two leptons from the $\beta$ decays of the two $W$ 's, and two $b$ quarks - the dilepton signature which has been the mainstay of all top searches. The net acceptance of events is $0.8 \%$. This acceptance is the result of its small branching ratio $\left(4.8 \%\right.$ for $\left.\ell \ell^{\prime}\right)$, the stringent requirements on the identification of each lepton and the requirement of a substantial missing iransverse energy (the neutrinos) in order to suppress backgrounds from electromagnetic pair production. If one might also require the existence of a $b$ quark in the event, the SVX information would be used to tag the $b$ quark jet. Its tagging efficiency is estimated to be about $30 \%$. Therefore, the total acceptance for $\ell \ell^{\prime}+b$ will be $0.2 \%$.

The dilepton signature provides for strong suppression of backgrounds of conventional (non-top) origin, but it does not provide a good measurement of the quark mass which would have produced the lepton pair. The problem is that the two undetected neutrinos carry away 
half of the mass energy in the top decay. Depending on the ingle between these neutrinos in a given went, the invariant mass of the dileptons from a given top mass is smeared over a broad spectrum as shown in Figure 20 for Monte-Carlo-simulated decays of a $120 \mathrm{GeV} / \mathrm{c}^{2}$ top quark.

To obtain a better estimate of the $t$ quark mass in each event, we have devised an analysis [2] which can identify $b$ quarks and reconstruct the decay $t \rightarrow W b$ with the CrTC track information. Therefore, this technique is additional capacity for tagging the $b$ quark with the SVX system.

The strategy begins with a sample of all collisions which contain a $W$ boson (observed through its $\beta$ decay) and one or more hadron jets (clusters of strongly interacting particles which signal a high-energy quark). It is too complex to fully reconstruct a $B$ meson through its hadronic decay mode in a $b$ quark jet. We have shown, however, that it is possible to identify jets originating in a $b$ quark by partially reconstructing the characteristic signature $D^{*} \rightarrow D \pi$, which accompanies most such events, and using it to actually measure an effective mass for the $b$ quark. The decay chain is:

$$
\begin{array}{rr}
b \rightarrow \overline{B^{0}}(\bar{d} b) & {[37.5 \%]} \\
\overline{B^{0}} \rightarrow D^{*+}(2010)+X & \\
D^{*+} \rightarrow D^{0}+\pi^{+} & {[55 \%]}
\end{array}
$$

where $X$ will be $\rho^{-}[1.8 \%]$ or $\ell^{-} \bar{\nu}_{\ell}(\ell=e$ or $\mu)$ [9.8\%] [30].

This partial reconstruction was first used in identifying $B$ mesons in $e^{+} e^{-}$collisions [31]. It relies on the useful fact that a $B$ meson usually decays into a $D^{*}$ meson (an excited state of the charm meson $D$ ), and the $D^{*}$ usually decays via $D^{*} \rightarrow D \pi$ near threshold. This last $\pi$ meson is the key to this analysis. It is produced nearly at rest in the $D^{*}$ frame, and can be used to measure the $D^{*}$ vector momentum:

$$
\vec{P}_{D^{*}} \sim \frac{M_{D^{*}}}{M_{\pi}} \vec{P}_{\pi}
$$

We thus start with the lowest energy particle in a hadron jet, and test for consistency with the $B$ decay topologies such as

$$
\begin{array}{ll}
B \rightarrow D^{*}+\rho & (B r \sim 0.018) \\
B \rightarrow D^{*}+\ell \nu & (B r \sim 0.098)
\end{array}
$$

We then partially reconstruct the $D^{*}$, and obtain a measure of the rest mass of the original $b$ quark. 


$$
\text { Simulation Study }(\mathrm{A}): \overline{B^{0}} \rightarrow D^{*+}+\rho^{-}[2]
$$

The branching ratio of the decay $\overline{B^{0}} \rightarrow D^{*+}+\rho^{-}$is $1.8 \%$. However, we see a characteristic relationship that the two charged pions have opposite signs in secondary decays.

$$
\begin{array}{ll}
D^{*+} \rightarrow D^{0} \pi^{+} & {[55 \%]} \\
\rho^{-} \rightarrow \pi^{-} \pi^{0} & {[100 \%]}
\end{array}
$$

Thus the first step was to find unlike-sign charged tracks in a hadron jet. The momentim of the charged pion from the $\rho$ decay is usually larger than the momentum of the charged pion in the $D^{*}$ decay (in the lab frame). Therefore we assign the labels $\pi_{\text {fast }}$ and $\pi_{\text {slow }}$, to the $\pi^{-}$and $\pi^{+}$, respectively. In order to test this technique, we have employed ISAJET to generate $b$ jets and $u / d$ jets with $P_{T}$ (parton) $\geq 15 \mathrm{GeV} / \mathrm{c}$.

To find a pair of $\pi^{+} \pi^{-}$tracks, we first reconstructed a jet based on the CDF algorithm (a fixed cone size of 0.7 ) and calculated the jet momentum. All tracks in the jet were assumed to be pions and the momenta were boosted into the center of mass system of the jet (hereafter, referred to as the jet rest frame). Figures $21 \mathrm{a}$ and $21 \mathrm{~b}$ show the momentum distributions of $\pi_{\text {fast }}$ and $\pi_{\text {slow }}$, respectively, in the $B$-meson rest frame (solid) and jet rest frame (circle). Now we searched for all unlike-sign track pair $\left(P_{T}(\right.$ track $\left.) \geq 0.3 \mathrm{GeV} / \mathrm{c}\right)$ in a jet and assigned $\pi_{\text {fast }}$ and $\pi_{\text {slow }}$ to tracks satisfying the following conditions:

$$
\begin{aligned}
& 0.25 \mathrm{GeV} / \mathrm{c} \leq\left|\vec{P}^{*}\left(\pi_{\text {fast }}\right)\right| \leq 2.5 \mathrm{GeV} / \mathrm{c} \\
& 0.05 \mathrm{GeV} / \mathrm{c} \leq\left|\vec{P}^{*}\left(\pi_{\text {slow }}\right)\right| \leq 0.25 \mathrm{GeV} / \mathrm{c}
\end{aligned}
$$

where $\vec{P}^{*}$ is the momentum in the jet rest frame. We also require $\cos \Delta \theta^{*}<-0.5$ where $\Delta \theta^{*}$ is the opening angle between $\vec{P}^{*}\left(\pi_{f a s t}\right)$ and $\vec{P}^{*}\left(\pi_{\text {slow }}\right)$. This is because two charged pions are likely in opposite hemispheres.

About $30 \%$ in the $D^{*} \rho$ jet sample has no candidates for unlike-sign track pairs. This is strongly correlated with the minimum $P_{T}($ track $)$ cut for tracks in a jet. About $77 \%$ of the remaining jets contain the true track pair. In jets with more than one track pair, we simply select the track pair with the maximum value of $\Delta \theta^{*}$. We find that $90 \%$ of the jets with genuine (i.e. from the $b$ quark decay) track pairs will be selected. Figure 22 shows the invariant mass distributions of the $D^{*} \pi$ system by using partial reconstruction for the two cases of genuine pair assignment (Figure 22a) and our selection criteria (Figure 22b). Figure $22 \mathrm{~b}$ contains about $70 \%$ of the total jets in Figure $22 \mathrm{a}$. 
After finding the best combination of unlike-sign tracks, we calculated an expected $\pi^{0}$ momentum:

$$
\vec{P}\left(\pi^{0}\right)_{\text {expected }}=\vec{P}(j e t)-\vec{P}\left(D^{*}\right)-\vec{P}\left(\pi_{f a s t}\right)
$$

We then tried to find a $\pi^{0}$ by looking for EM calorimeter energy satisfying the following condition.

$$
E M F=\frac{E(E M)}{E(E M)+E(H A D)}>0.9\left(E_{\text {threshold }}=0.2 \mathrm{GeV}\right)
$$

where $E(E M)$ and $E(H A D)$ are the tower energies of electromagnetic (EM) and hadronic (HAD) calorimeters. Figure 23 shows $\cos \Delta \theta^{*}$ distribution for all EM towers $(E M F>0.9)$ within a cone of 0.7 around a jet. Here $\Delta \theta^{*}$ is the opening angle between $\vec{P}^{*}\left(\pi^{0}\right)_{\text {expected }}$ and $\vec{P}^{*}(E M)$ in the jet rest frame. A clear enhancement near $\cos \Delta \theta^{*}=1$ indicates a candidate for the $\pi^{0}$ tower. We selected towers satisfying the condition:

$$
\cos \Delta \theta^{*} \geq 0.707\left(45^{\circ} \text { cone }\right)
$$

Finally, we required $E_{T}(E M) \geq 0.5 \mathrm{GeV}$ which is the lowest energy for electron/photon detection with the CDF detector.

If a $\pi^{0}$ candidate was found, we reconstructed $M_{D^{*} \rho}$ and $M_{\rho}$ by,

$$
\begin{aligned}
M_{D^{*} \rho} & =M\left(\vec{P}\left(\pi_{f a s t}\right), \vec{P}\left(\pi^{0}\right), \vec{P}\left(D^{*}\right)\right) \\
M_{\rho} & =M\left(\vec{P}\left(\pi_{f a s t}\right), \vec{P}\left(\pi^{0}\right)\right)
\end{aligned}
$$

Figure 24 shows the resulting mass spectra for Monte-Carlo-simulated $b$ jets with $B \rightarrow D^{*} \rho$ (Figure 24a) and for the $\pi^{-} \pi^{0}(" \rho ")$ system (Figure 24b). Similarly, Figure 25 shows the resulting mass spectra for Monte Carlo simulated $u / d$ quark jets (Figure 25a) and for $\pi^{-} \pi^{0}$ (" $\rho$ ") particles (Figure 25b). The final reconstruction efficiency is $29 \%$ for $D^{*} \rho$ jets, and a fake rate of $2.7 \%$ from $u / d$ jets. A mass cut of $M_{D^{*} \rho}>4.6 \mathrm{GeV} / \mathrm{c}^{2}$ yielded an acceptance of $24 \%$ for $b$ jets and $0.6 \%$ for $u / d$ jets - a background rejection factor of 40 .

Using this result in conjunction with the high $P_{T}$ lepton from the $W$ boson, the transverse mass of the top quark is calculated as

$$
M_{T}^{2}=\left(\sqrt{\left|\vec{P}_{T}(\ell B)\right|^{2}+M(\ell B)^{2}}+P_{T}(\nu)\right)^{2}-\left|\vec{P}_{T}(\ell B+\nu)\right|^{2} .
$$

Figure 26 shows the mass spectrum when we identify the $B$ meson. The sharp peak in the mass distribution at the top mass is characteristic of this technique. 
Table 2: Acceptance of top quark events by $P_{T}$ and $\eta$ cuts for $D^{*} \rho$ mode

\begin{tabular}{||c|c|c|c||}
\hline$M_{\text {top }}$ & $P_{T}$ & \multicolumn{2}{|c||}{$P_{T}$ Cuts plus } \\
\cline { 3 - 4 } $\mathrm{GeV} / \mathrm{c}^{2}$ & Cuts & $|\eta|<1.0$ & $|\eta|<2.0$ \\
\hline \hline 100 & .59 & .24 & .44 \\
\hline 120 & .70 & .28 & .53 \\
\hline 150 & .78 & .33 & .64 \\
\hline 180 & .87 & .34 & .66 \\
\hline
\end{tabular}

Using the reconstruction efficiency obtained in the this study, we estimated the acceptance for the following decay chain.

$$
\begin{array}{rc}
t \rightarrow W^{+}+b & {[100 \%]} \\
W^{+} \rightarrow \ell^{+}+\nu_{\ell} & {[22 \%]} \\
b \rightarrow \overline{B^{0}}(\bar{d} b) & {[37.5 \%]} \\
\overline{B^{0}} \rightarrow D^{*+}+\rho^{-} & {[1.8 \%]} \\
D^{*+} \rightarrow D^{0}+\pi^{+} & {[55 \%]} \\
\rho^{-} \rightarrow \pi^{-}+\pi^{0} & {[100 \%]}
\end{array}
$$

We set $P_{T}$ cuts for the $\ell_{W}$ and the $b$ quark to be $20 \mathrm{GeV} / \mathrm{c}$ and $15 \mathrm{GeV} / \mathrm{c}$, respectively. If $M_{\text {top }}=120 \mathrm{GeV} / \mathrm{c}^{2}$, the total acceptance in the central region $(|\eta|<1)$ will be $2(t$ or $\bar{t}) \times 0.22(W \rightarrow \ell \nu) \times 0.375\left(b \rightarrow \overline{B^{0}}\right) \times 0.018\left(\overline{B^{0}} \rightarrow D^{*} \rho\right) \times 0.55\left(D^{*} \rightarrow D^{0} \pi\right) \times$ $0.28\left(P_{T}\right.$ Cuts $\left.+|\eta|<1.0\right) \times 0.90$ (identification efficiency for $\left.\ell_{W}\right) \times 0.24$ (reconstruction efficiency of $B$ meson) $=0.01 \%$. We expect to see $\approx 0.3$ events in the next collider run (100 $\left.\mathrm{pb}^{-1}\right)$. The acceptances for various top quark mass are summarized in Table 2.

$$
\text { Simulation Study }(\mathrm{B}): \overline{B^{0}} \rightarrow D^{*+}+\ell^{-}+\bar{\nu}_{\ell}[3]
$$

We first made the MC samples for four top masses $\left(100,120,150\right.$ and $\left.180 \mathrm{GeV} / \mathrm{c}^{2}\right)$ in the following decay chain:

$$
\begin{array}{rr}
t \rightarrow W^{+}+b & {[100 \%]} \\
W^{+} \rightarrow \ell^{+}+\nu_{\ell} & {[22 \%]}
\end{array}
$$


Table 3: Acceptance of top quark events by $P_{T}$ and $\eta$ cuts for $D^{*} \ell \nu$ mode

\begin{tabular}{||c|c|c|c||}
\hline$M_{\text {top }}$ & $P_{T}$ & \multicolumn{2}{|c||}{$P_{T}$ Cuts plus } \\
\cline { 3 - 4 } $\mathrm{GeV} / \mathrm{c}^{2}$ & Cuts & $|\eta|<1.0$ & $|\eta|<2.0$ \\
\hline \hline 100 & .48 &. $\mathrm{i} 8$ & .33 \\
\hline 120 & .61 & .21 & .42 \\
\hline 150 & .68 & .24 & .48 \\
\hline 180 & .72 & .25 & .51 \\
\hline
\end{tabular}

$$
\begin{array}{rc}
b \rightarrow \overline{B^{0}} & {[37.5 \%]} \\
\overline{B^{0}} \rightarrow D^{*+}+\ell^{-}+\bar{\nu} & {[9.8 \%]} \\
D^{*+} \rightarrow D^{0}+\pi^{+} & {[55 \%]}
\end{array}
$$

The selection cuts are (a) $P_{T}\left(\ell_{W}\right)>20 \mathrm{GeV} / \mathrm{c}$, (b) $P_{T}\left(\ell_{B}\right)>2 \mathrm{GeV} / \mathrm{c}$ and (c) $P_{T}\left(\pi_{D^{*}}\right)$ $>0.3 \mathrm{GeV} / \mathrm{c}$. We studied two rases: (a) $|\eta|<1$ and (b) $|\eta|<2$. Table 3 summarizes the acceptances in these two regions. This clearly shows that twice as many events can be accepted baced on momentum cuts if the intermediate angle region is included.

For the decay mode $\overline{B^{0}} \rightarrow D^{*+}+\ell^{-}+\bar{\nu}_{\ell}$, the $\bar{\nu}_{\ell}$ is lost. Therefore, we partially calculate the 4 -momentum of the $B^{0}$ meson:

$$
P_{B^{0}}^{\mu} \approx P_{D^{\bullet+}}^{\mu}+P_{l^{-}}^{\mu}
$$

Thus the mass of the $\overline{B^{0}}$ meson $\left(M_{B}\right)$ can partially be estimated.

The kinematics of the decay products of the $B$ meson in the rest frame of the $B$ meson were studied: $\cos \theta^{*}, \vec{P}_{\ell_{B}}^{*}$ and $\vec{P}_{\pi_{D^{*}}^{*}}$. The $\theta^{*}$ is the opening angle between the $\pi_{D^{*}}$ and the $\ell_{B}$. Figure 27a shows the simulated distribution of $\cos \theta^{*}$ peaking at -1.0 . The $\pi_{D^{*}}$ will proceed in virtually the same direction as the $D^{*}$. The momentum distributions for $\pi_{D^{*}}$ and $\ell_{B}$ are shown in Figures 27b and 27c. Figure 28 shows the partially-reconstructed mass of the $B$ meson.

In order to select the signal, we applied the same cut values for $P_{\ell}^{*}, P_{\pi}^{*}$ and $\cos \theta^{*}$ as in $D^{*} \rho$ mode. We also applied a cut of $M_{B}>3.0 \mathrm{GeV} / \mathrm{c}^{2}$. Table 4 summarizes the acceptances of the $B$ mesons in the M.C. samples.

Once we obtain $B$ meson information, we can calculate the transverse mass of the top quark in the same manner as in $D^{*} \rho$ mode. It should be noted that the $P_{T}$ of the $\nu_{W}$ 
Table 4: Acceptance of $B$ meson in top quark events by $P_{\ell}^{*}, P_{\pi}^{*}, \cos \theta^{*}$ and $M_{B}$ cuts in $B$ meson rest frame for $D^{*} \ell \nu$

\begin{tabular}{||c|c|c||}
\hline$M_{\text {top }}\left(\mathrm{GeV} / \mathrm{c}^{2}\right)$ & $P_{\ell}^{*}, P_{\pi}^{*}, \cos \theta^{*}$ & $M_{B}>3.0 \mathrm{GeV} / \mathrm{c}^{2}$ \\
\hline \hline 100 & .67 & .81 \\
\hline 120 & .66 & .82 \\
\hline 150 & .64 & .82 \\
\hline 180 & .64 & .83 \\
\hline
\end{tabular}

Table 5: Top quark acceptances for the $D^{*} \ell \nu$ mode

\begin{tabular}{||c|c|c||}
\hline$M_{\text {top }}$ & \multicolumn{2}{|c||}{ All Cuts } \\
\cline { 2 - 3 } $\mathrm{GeV} / \mathrm{c}^{2}$ & $|\eta|<1.0$ & $|\eta|<2.0$ \\
\hline \hline 100 & .104 & .197 \\
\hline 120 & .132 & .259 \\
\hline 150 & .142 & .289 \\
\hline 180 & .136 & .297 \\
\hline
\end{tabular}

was replaced with a vector sum of $\nu_{W}$ and $\nu_{B}$. This is because only the total $E_{T}$ can be measured experimentally.

Figure $29 \mathrm{a}$ shows the reconstructed mass of the top quark $\left(M_{\text {top }}=120 \mathrm{GeV} / \mathrm{c}^{2}\right)$ with all cuts and $|\eta|<2.0$, where all cuts are (a) $P_{T}\left(\ell_{W}\right)>20 \mathrm{GeV} / \mathrm{c}$, (b) $P_{T}\left(\ell_{B}\right)>2 \mathrm{GeV} / \mathrm{c}$, (c) $P_{T}\left(\pi_{D^{*}}\right)>0.3 \mathrm{GeV} / \mathrm{c}$, (d) $P_{\ell}^{*}, P_{\pi}^{*}, \cos \theta^{*}$ cuts and (e) $M_{B}>3.0 \mathrm{GeV} / \mathrm{c}^{2}$. Figure $29 \mathrm{~b}$ is the same as Figure 29a, but with $|\eta|<1.0$. Table 5 summarizes the acceptances for various top quark masses.

The total acceptance in the central region $(|\eta|<1)$ if $M_{\text {top }}=120 \mathrm{GeV} / \mathrm{c}^{2}$ will be $2(t$ or $\bar{t}) \times 0.22(W \rightarrow \ell \nu) \times 0.375\left(b \rightarrow \overline{B^{0}}\right) \times 0.098\left(\overline{B^{0}} \rightarrow D^{*} \ell \nu_{\ell}\right) \times 0.55\left(D^{*} \rightarrow\right.$ $\left.D^{0} \pi\right) \times 0.45$ (identification efficiency for two leptons) $\times 0.132$ (All Cuts $+|\eta|<1$ ) $=0.05 \%$. We expect to see $\approx 1.6$ events in the next collider run $\left(100 \mathrm{pb}^{-1}\right)$. This is smaller by a factor of four compared to the $\ell \ell^{\prime}+b$ (SVX) mode. However, this technique will provide better accuracy to determine the top quark mass. 
Table 6: Number of top quark events expected at $100 \mathrm{pb}^{-1}(|\eta|<1)$

\begin{tabular}{||c|c||c|c||}
\hline$M_{\text {top }}\left(\mathrm{GeV} / \mathrm{c}^{2}\right)$ & $\sigma_{t \bar{t}}(\mathrm{pb})$ & $D^{*} \rho$ & $D^{*} \ell \nu$ \\
\hline \hline 100 & 90 & 0.78 & 3.8 \\
\hline 120 & 30 & 0.30 & 1.6 \\
\hline 150 & 10 & 0.12 & 0.6 \\
\hline 180 & 3 & 0.06 & 0.2 \\
\hline
\end{tabular}

The background for the $D^{*} \ell \nu$ mode is expected to be very small because of (a) the existence of a lepton $\left(\ell_{B}\right)$ in the jet; (b) requirement of unlike-sign combination of the $\ell$ and $\pi$; (c) kinematical constraints for $\ell$ and $\pi$; (d) the reconstructed mass cut on the $B$ meson.

\section{Summary}

We developed a partial reconstruction technique to tag the $b$ quark for the hadron collider experiments. This technique should provide an additional capability for finding the top quark with the SVX system in the $1992-93$ runs $\left(100 \mathrm{pb}^{-1}\right)$ as well as to study $b$ physics. Table 6 shows the number of accepted events from the $D^{*} \rho$ and $D^{*} \ell \nu$ modes for the central region with $100 \mathrm{pb}^{-1}$ of the data.

\section{A.3 $\Lambda_{b}$ Search}

\section{Introduction}

The first observation of the $\Lambda_{b} \rightarrow \Lambda^{0} J / \psi$, was reported by UA1 at the $\bar{p} p$ collider at CERN, where the $\Lambda_{b}$ is the lightest baryon containing a $b$ quark $(u d b)$. The mass was measured to be $5640 \pm 50$ (stat.) \pm 30 (sys.) $\mathrm{MeV} / \mathrm{c}^{2}$ [32]. In this process, the secondary decay $\Lambda^{0} \rightarrow p \pi(B r=64 \%)$ was reconstructed due to the typical $V^{0}$ decay associated with the long $\Lambda^{0}$ lifetime.

An equivalent search was also made by CDF using $2.6 \mathrm{pb}^{-1}$ if the di-muon events collected in the 1988-89 run [33]. The decay of the $J / \psi$ into $\mu^{+} \mu^{-}$provides a relatively clean 
trigger signature in hadronic collisions. There were $52 \pm 12 \Lambda^{0}$ signals associated with the $J / \psi$ particle. The mass spectrum is given in Figure 30. The resulting $\Lambda^{0} J / \psi$ invariant mass spectrum is shown in Figure 31. We estimated a background level of $0.7 \pm 0.2$ events per 40 $\mathrm{MeV} / \mathrm{c}^{2}$ bin in the region between $5300 \mathrm{MeV} / \mathrm{c}^{2}$ and $6000 \mathrm{MeV} / \mathrm{c}^{2}$. We don't see any evidence for this decay. This led to the conclusion of $F\left(\Lambda_{b}\right) \cdot B r\left(\Lambda_{b} \rightarrow \Lambda^{0} J / \psi\right)<0.70 \times 10^{-3}$ at $90 \%$ confidence level compared to the observed value from UA1 of $1.8 \pm 0.6$ (stat.) \pm 0.9 (sys.) $\times 10^{-3}$.

In the 1992 run, we will collect $25 \mathrm{pb}^{-1}$ of data with the a new amplifiers on CTC which allows us to measure the pulse height for each track. Therefore, we can separate the charged particles $(p, K$ and $\pi)$ in a certain momentum range using the $d E / d x$ method. It should substantially reduce the background events in the $\Lambda^{0}$ selection compared to Figure 30.

\section{Work in Progress}

The particle identification working group was formed to work on the hardware and software related to the $d E / d x$ measurement. We are working on [4]

- extracting minimum bias events for the test of the CTC hardware;

- writing a detector simulation code for $d E / d x$;

- studying the detection efficiency with the $d E / d x$ measurement using the ISAJET program.

Figure 32 shows a preliminary plot of $d E / d x$ (in ADC) vs $p$ for tracks in the event sample. It should be noted that the calibration of the amplifiers is not completed. Therefore, each band is very broad. However, we clearly see two bands (expected to be $\pi$ and $K / p$ ). The preliminary conclusion from the plot is the momentum range for the particle identification is below $1 \mathrm{GeV} / \mathrm{c}$. We are still working on calibrating the hardware to find the performance of the $d E / d x$ measurement by the CTC system.

The simulation work began at the beginning of September. However, we found a problem in the ISAJET code; it did not generate $\Lambda_{b}$ particles. We have recently identified the bug in the code and fixed it. Therefore, we are able to study the $d E / d x$ performance on its particle identification for this $\Lambda_{b}$ search. It should be noted that the simulation in Ref. [33] is made with a simple Monte Carlo program. 


\section{B. 1991 Test-Beam Data Analysis}

Following successful tests/studies of the noise immunity of the forward hadron (FHA) calorimeter, we had the test-beam run in 1991. The data analysis was recently completed to provide the basic information of the detector performance for the 1992 collider run [34]. This work was carried out by Profs. Kamon and McIntyre, Drs. Wolinski and Scarpine, and Messrs. Lamecker, Keeble (graduate student) and Wahl (undergraduate student). We summarize the performance of the FHA calorimeter with the configuration in the 1992 collider run.

\section{Introduction}

Two separate testbeam (TB) calibration runs were performed since the last collider run. In the 1990 testbeam run, we calibrated the forward hadron (FHA) calorimeter with a preliminary version of the new front-end electronics, general purpose amplifier (GPA) cards. The primary purpose of this run was to determine (a) $V_{o}$ (the operational high voltage for the 1992 run) and (b) $C_{f b}$ (the feedback capacitance of the FHA GPA cards). These quantities were determined to be $V_{o}=2000$ Volts and $C_{f b}=56 \mathrm{pF}$ [35]. During the second testbeam run (June through December 1991) the goal was to determine the absolute energy calibration for the gas calorimeters using the same configuration expected in the 1992 CDF collider run: the final version of the GPA cards with an integration gate width of $850 \mathrm{nsec}$ and the new operational high voltage. In view of the expected peak luminosity of $10^{31} \mathrm{~cm}^{-2} \mathrm{sec}^{-1}$ with $3.5 \mu$ sec between beam crossings in 1992-93 collider run, these changes were necessary to prevent the gas calorimeters from entering glow mode. The changes made to the FHA system are summarized in Table 7.

\section{Testbeam Setup}

The forward calorimeters (FEM and FHA) and its gas flow/pressure control system in the testbeam area were virtually identical to the TNE (Top North East) quadrant of the $\mathrm{CDF}$ experiment at $\mathrm{B} 0$. The only major difference between the testbeam and $\mathrm{B} 0$ is that the testbeam FHA quadrant utilizes steel absorber plates that are only half as high as those at B0. Thus, hadronic showers can only be studied in the bottom portion of the testbeam FHA quadrant. 
Table 7: Summary of changes in FHA system at the 1991 testbeam.

\begin{tabular}{|c|c|c|c|c|}
\hline \multicolumn{2}{|c|}{ Iteın } & 1988-89 Run & $\begin{array}{c}1991 \text { TB Run } \\
\text { (1992 Run) }\end{array}$ & Comments \\
\hline \multicolumn{2}{|l|}{ Chamber HV } & $2100 \mathrm{~V}$ & $2000 \mathrm{~V}$ & $\mathrm{G}_{2100} / \mathrm{G}_{2000}=2.3$ \\
\hline Amp. Card & $\begin{array}{l}\text { FHA } \\
\text { FHX }\end{array}$ & $\begin{array}{l}\text { CARROT } \\
\text { HGW }\end{array}$ & $\begin{array}{l}\text { GPA } \\
\text { HGW }\end{array}$ & $\begin{array}{l}200 \mathrm{pF} \rightarrow 56 \mathrm{pF} \\
\text { unchanged }\end{array}$ \\
\hline Gate Width & & $1.6 \mu \mathrm{sec}$ & $850 \mathrm{nsec}$ & \\
\hline GG Monitor & $\begin{array}{l}\text { \#Tubes/Quad. } \\
\text { Tube HV } \\
\text { Tube Gain }\end{array}$ & $\begin{array}{l}3 \\
2100 \mathrm{~V} \\
\approx 1 \mathrm{pC}\end{array}$ & $\begin{array}{l}4 \\
1950 \mathrm{~V} \\
\approx 300 \mathrm{fC}\end{array}$ & \\
\hline
\end{tabular}

The data acquisition configuration was also identical to the B0 environment: VAXMEP-MX-RABBIT. Pedestals were subtracted in the MX. However, there were no further online corrections to the data performed by the MX. This allowed us to do a careful study of the charge injection (EQI) and gas gain corrections and apply those to the data in the offline analysis.

The gas gain monitoring system is set up to be similar to the one in B0. We have a total of four Brandeis (BRD) tubes (two tubes in series in the gas input line and two tubes in the gas output line): IN1, IN2, OUT1 and OUT2. The gas gain data acquisition program (GASDAQ) was kept running continuously, even when beam was not present.

Gas density monitoring was accomplished using a pressure transducer in the enclosure and 10 temperature transducers (AD592) placed on the detector and BRD tubes. The locations were determined by an extensive study of the temperature map on the FHA in the early stage of the $1991 \mathrm{~TB}$ run. In this note, the density of the gas is clefined as

$$
\rho=\frac{\text { Pressure }(\mathrm{mBar})}{\text { Temperature }(\mathrm{K})}
$$

Details of the temperature transducer calibration and stability are discussed in Ref. [36].

We also monitored the oxygen contamination in the argon-ethane gas exiting the detectors. During the calibration runs, the $\mathrm{O}_{2}$ level was stable at about $480 \mathrm{ppm}$. 


\section{Energy Scale Calibration}

We define the FHA calorimeter absolute energy scale as the response per momentum for a single incident pion (at $150 \mathrm{GeV} / \mathrm{c}$ ) punching through the FEM calorimeter. This response is measured for the nominal FHA condition, where nominal is defined as (a) nominal high voltage: $V_{o}=2000$ Volts; (b) nominal density: $\rho_{o}=3.338 \mathrm{mBar} / \mathrm{K}$ - the average density measured in the B0 collision hall during a two week period in the 1988-89 collider run; (c) nominal gas mixture: Argon-Ethane 50\%-50\%, bubbled through an isopropanol bath maintained at $10^{\circ} \mathrm{C}$. With a fixed high voltage and gas mixture, the calorimeter response is a function only of the gas density.

Energy Measurement. All testbeam data, except high voltage scans, are taken under high voltage feedback, described elsewhere [37]. There are a large number of so-called standard runs, taken with fixed beam and detector conditions, and used for energy calibration and study of the correlation between gas density and gas gain.

We apply the same analysis program (JETCLU) for the clustering used in the collider data with the texas tower cleanup program (FILT_GAS [38]). The momentum of each particle is determined from single wire drift chamber (SWDC) data. The cluster energy distribution given by JETCLU is fitted to a Gaussian without any cut. Then a 3- $\sigma$ cut around the mean value is applied for the 2 nd iteration of the Gaussian fit to obtain the mean (the detector response) and the width (the resolution).

The raw measurement of the hadron shower energy on the FEM and FHA pads is made by JETCLU with FILT_GAS and EQI correction. For the anode energy, we add up all anode channels (FEA or FHX). It should be noted that the 1988-89 collider run energy scales are used in this analysis. The events used for the FHA energy calibration are required to have total FEM anode wire energy less than $0.15 \mathrm{GeV}$. This cut assures that the pions are punch-through particles in FEM.

Since HV feedback was on, we have to remove the feedback effect to study the relation between the calorimeter response and the gas density. We parameterize the detector response as an exponential function of high voltage:

$$
E=E_{o} \exp \left(\frac{V-V_{o}}{\alpha}\right)[\mathrm{GeV}]
$$

where $E_{o}$ is the response at the nominal voltage $V_{o}(=2000 \mathrm{~V})$. We find $\alpha=120.8 \pm$ 0.7 Volts. This is consistent with the value measured (119.1) in the 1990 testbeam run. For 
the remaining measurements, we use this result to correct the calorimeter response to the nominal voltage.

The longitudinal profile of $150-\mathrm{GeV} / \mathrm{c}$ pion showers in the FHA is fitted with the sum of two exponential functions. We estimated the correction factor $(D L C)$ to the observed energy due to the low gain of Chamber 26 and dead layer in Chamber 27 to be $1.0028 \pm$ $0.0018^{1}$. The correction factor $(L C)$ for longitudinal energy leakage is calculated to be 1.023 \pm 0.006 .

$E / p$ vs $\rho$. The measured FHA energy (without $D L C$ and $L C$ ) described in the previous section is correlated to the gas density. Figures $33 \mathrm{a}$ and $33 \mathrm{~b}$ show plots of $E / p$ vs $\rho$ for par' and anode energies, respectively. The response of a gas calorimeter vs. gas density is ral:ameterized as follows:

$$
E / p=(E / p)_{\circ}\left(\rho / \rho_{o}\right)^{-S}
$$

Here $\rho_{o}=3.338 \mathrm{mBar} / \mathrm{K}$. We obtained

$$
\begin{aligned}
\left(E_{\text {pad }} / p\right)_{0} & =1.200 \pm 0.003 \text { and } S_{\text {pad }}=7.82 \pm 0.14 \\
\left(E_{\text {anode }} / p\right)_{0} & =0.3031 \pm 0.0006 \text { and } S_{\text {anode }}=7.57 \pm 0.14
\end{aligned}
$$

A value deviating from unity represents a correction factor to the 1989-89 energy scale. Therefore, the new pad energy scale for the 1992-93 run is

$$
\begin{aligned}
S C L_{\text {pad }} & =13.72 \times \frac{1}{\left(E_{\text {pad }} / p\right)_{\circ} \times D L C \times L C} \\
& =11.15 \pm 0.07[\mathrm{MeV} / \mathrm{ADC}] .
\end{aligned}
$$

Similarly, the new anode wire energy scale is

$$
\begin{aligned}
S C L_{\text {anode }} & =3.29 \times \frac{1}{\left(E_{\text {anode }} / p\right)_{\circ} \times D L C \times L C} \\
& =10.58 \pm 0.07[\mathrm{MeV} / \mathrm{ADC}]
\end{aligned}
$$

${ }^{1}$ FHA has a total of 27 chambers. 


\section{Gas Gain Analysis}

The gas gain is a function of $\mathrm{HV}$ and $\rho$ which is parameterized as

$$
G_{\text {meas }}=G_{o, \text { meas }} \exp \left(\frac{V-V_{o}}{\alpha_{G}}\right)\left(\rho / \rho_{o}\right)^{-S_{G}} \quad[\mathrm{fC}] .
$$

We find ${ }^{2} \alpha_{G}=112.7 \pm 0.7$ Volts and $S_{G}=7.01 \pm 0.25$ at $V_{o}=1950$ Volts as shown in Figure 34. The value of $\alpha_{G}$ is consistent with $113.0 \pm 0.3$ in the 1990 TB measurement. These numbers are used for the HV feedback system in the 1992 run.

\section{Energy Linearity and Resolution}

Since the Fermilab beam line can provide pion beam momenta from $10 \mathrm{GeV} / \mathrm{c}$ to 230 $\mathrm{GeV} / \mathrm{c}$, we measured the linearity and resolution of the FHA calorimeter at a fixed position. Unfortunately, the $10 \mathrm{GeV} / \mathrm{c}$ and $25 \mathrm{GeV} / \mathrm{c}$ data quality was poor. Therefore, $50-230 \mathrm{GeV} / \mathrm{c}$ data is used in this analysis.

Figure 35 shows $E / p$ vs $p$ from the 1991 testbeam data (corrected for shower energy leakage out of the far end of the FHA). We expect the FHA to exhibit a linear energy response as a function of momentum but see some nonlinearity in the lower momentum region. This effect is under study. Note however that Figure 35, fitted only between 100 and $200 \mathrm{GeV} / \mathrm{c}$, does not indicate nonlinearity. Figure 36 shows $\sigma_{E / p} /(E / p)$ vs $1 / \sqrt{p}$. The data points are fitted with

$$
(\sigma / E)^{2}=C^{2}+\frac{S^{2}}{p}+\frac{N^{2}}{p^{2}}
$$

where $C=$ constant term, $S=$ statistical fluctuation term of shower sampling, $N=$ electronics noise term. We find

$$
\begin{aligned}
& C=0.048 \pm 0.013 \\
& S=1.306 \pm 0.076 \\
& N=0.043 \pm 9.7
\end{aligned}
$$

Note that the $N$ term is significantly lower than the value obtained during the previous testbeam run $(\approx 2.5)$. This difference is due to the new grounding straps attached during this testbeam run.

\footnotetext{
${ }^{2}$ Two of the gas gain tubes $(I N 1$ and $I N 2)$ had smaller gas gains than expected. Also, the calibration number for tube OUT2 was lost by accident [40]. Therefore, we used the gas gain of OUT1 tube as a reference.
} 


\section{Glow Mode Limit}

In order to estimate the limit of entering the glow mode, we took data with a highintensity pion beam at a high gain: (a) $230 \mathrm{GeV} / \mathrm{c} \pi^{+}$; (b) $123 \mathrm{~K}$ particles during $20 \mathrm{sec}$ with the period of $60 \mathrm{sec}$; (c) FHA at 2200 Volts. Under the conditions, a maximum dark current on Chamber 5 (near shower maximum) was $2.8 \mu \mathrm{A}$ and a total current for 27 chambers was $30 \mu \mathrm{A}$. Most importantly, the FHA chambers did not enter the glow mode regime.

Most of the shower would be contained within a $10 \mathrm{~cm} \times 10 \mathrm{~cm}$ area. Thus, about 7 anode wires are collecting the charge. Using the measured value instead of the calculation, the current per wire will be $400 \mathrm{nA}$. This is an approximate upper limit of FHA current draw without entering glow mode.

According to the measurement of the dark current (per chamber) at $2100 \mathrm{~V}$ in the 1988-89 run [41], we simply calculate the expected dark current in the 1992 run:

$$
\begin{aligned}
I_{\text {dark }}^{1992} & =I_{\text {dark }}^{1989} \times \frac{\mathcal{L}^{1992}}{\mathcal{L}^{1989}} \times \frac{G(2000 \mathrm{~V})}{G(2100 \mathrm{~V})} \\
& =2.17 \mu \mathrm{A} / \text { chamber },
\end{aligned}
$$

where $I_{\text {dark }}^{1989}=500 \mathrm{nA} \quad$ per chamber, $\quad \mathcal{L}^{1989}\left(\mathcal{L}^{1992}\right)=10^{30}\left(10^{31}\right) \mathrm{cm}^{-2} \mathrm{sec}^{-1} \quad$ and $G(2000 \mathrm{~V}) / G(2100 \mathrm{~V})=1 / 2.3$. Since each chamber contains 115 proportional tubes, the current per wire is $19 \mathrm{nA}$. Thus, the testbeam result seems to be $21(=400 / 19)$ times higher than what we expected in the 1992 run. The FHA chambers appear not to be in danger of entering glow mode at $2000 \mathrm{~V}$ at $\mathcal{L}=10^{31} \mathrm{~cm}^{-2} \mathrm{sec}^{-1}$.

\section{Aging Limit}

The aging limit for a chamber at $2.1 \mathrm{kV}$ has been measured with Ar/Ethane and isopropyl-alcohol [42]. There was no observed deterioration in the pulse height up to $Q_{a g i n g}$ $=0.3 \mathrm{C} \cdot \mathrm{cm}^{-1}$. The corresponding aging time limit is calculated to be

$$
T_{\text {aging }}=\frac{Q_{\text {aging }}}{q_{B B}}
$$

where $q_{B B}$ is the charge flow in the FHA chambers due to $\bar{p} p$ colliding beam events. This is given [41] as $q_{B B}=10.5 \times x \times \exp [(V-2100) / 120.8] \quad\left[\mathrm{pC} \cdot \mathrm{sec}^{-1} \cdot \mathrm{cm}^{-1}\right]$. Here $x$ is a factor of $10^{30} \mathrm{~cm}^{-2} \cdot \mathrm{sec}^{-1}$. Thus, if the Tevatron is operated at $\mathcal{L}=10^{31} \mathrm{~cm}^{-2} \cdot \mathrm{sec}^{-1}(x=$ 10), the FHA chambers will not be damaged for about 210 years at $2.0 \mathrm{kV}$. 
Summary from TB Data Analysis

From the analyses, we expect the following conditions of the FHA system for the 1992-93 run:

(i) $S C L: 11.15 \mathrm{MeV} / \mathrm{ADC}$ (FHA), $10.58 \mathrm{MeV} / \mathrm{ADC}$ (FHX);

(ii) No aging and glow mode problems are expected during the 1992-93 collider run;

(iii) The gas gain/HV feedback pararneters $(S, \alpha)$ are well determined so that the systematic error on measurement of the gas density and gas gain is minimized;

(iv) Improved noise immunity (reduction of coherent noise).

\section{Forward Hadron Calorimeter in the 1992 Collider Run}

The TAMU group modified the readout cables and grounding condition for the FHA at $\mathrm{B} 0$ before starting the 1992 collider run. This was necessary to improve the noise immunity. We also increased the number of temperature sensors to measure the gas temperature in order to minimize the systematic uncertainty in the gas gain measurement during the collider run. The FHA calorimeter is now stably in operation [44]. Table 8 provides a summary of the performance comparison for FHA system between two collider runs. This work was carried out by Profs. Kamon, McIntyre and Webb, Drs. Wolinski and Scarpine and Ms. David (Technician). We report the improvement of the noise immunity in the 1992 collider run.

\section{Electronic Noise Immunity}

In order to reduce the electronic noise on the FHA, two modifications were performed:

(i) Grounding straps were added to improve the noise immunity of the pads;

(ii) Flat ribbon cables, used for pad readout, were replaced by twisted-pair ribbon cables in an attempt to reduce cross-talk. 
Table 8: Performance Comparison of FHA System at B0

\begin{tabular}{|c|c|c|c|}
\hline & & 1988-89 run & 1992-93 run \\
\hline Tevatron & $\begin{array}{l}\text { \# bunches } \\
\mathcal{L}_{\text {peak }}\left[\mathrm{cm}^{-2} \cdot \mathrm{sec}^{-1}\right]\end{array}$ & $\begin{array}{r}6 \\
10^{30} \\
\end{array}$ & $\begin{array}{r}6 \\
10^{31} \\
\end{array}$ \\
\hline Charnbers & $\begin{array}{l}\text { HV } \\
G_{\text {anode }} \\
I_{\text {dark }} \text { [nA/layer] } @ \mathcal{L}_{\text {peak }} \\
\text { Aging Limit [years] } \\
\text { Glow Mode? }\end{array}$ & $\begin{array}{r}2100 \mathrm{~V} \\
7.73 \times 10^{3} \\
\approx 500 \text { (meas.) } \\
910 \\
\text { No } \\
\end{array}$ & $\begin{array}{r}2000 \mathrm{~V} \\
3.36 \times 10^{3} \\
\approx 2200 \text { (est.) } \\
210 \\
\text { No }\end{array}$ \\
\hline Gate Width [nsec] & & 1600 & 850 \\
\hline FHA Amp. Card & $\begin{array}{l}\text { Type } \\
\text { \#Cards } \\
\text { Gain [fC/ADC] } \\
<\sigma_{\text {ped }}> \\
\text { ADC Threshold } \\
S C L[\mathrm{MeV} / \mathrm{ADC}]\end{array}$ & $\begin{array}{r}\text { CARROT } \\
120 \\
7.63 \\
6 \text { counts } \\
10 \text { counts } \\
13.72 \\
\end{array}$ & $\begin{array}{r}\text { GPA } \\
120 \\
2.1364 \\
12 \text { counts } \\
20 \text { counts } \\
11.15 \pm 0.07 \\
\end{array}$ \\
\hline FHX Amp. Card & $\begin{array}{l}\text { Type } \\
\text { \#Cards } \\
\text { Gain [fC/ADC] } \\
<\sigma_{p e d}> \\
\text { ADC Threshold } \\
S C L[\mathrm{MeV} / \mathrm{ADC}] \\
\end{array}$ & $\begin{array}{r}\text { HGW } \\
40 \\
3.81 \\
3 \text { counts } \\
10 \text { counts } \\
3.29 \\
\end{array}$ & $\begin{array}{r}\text { HGW } \\
40 \\
3.8148 \\
4 \text { counts } \\
10 \text { counts } \\
10.58 \pm 0.07 \\
\end{array}$ \\
\hline$\sigma / E$ & $@ 100 \mathrm{GeV}$ & $10 \%$ & $14 \%$ \\
\hline GG Monitor & $\begin{array}{l}\text { \#Tubes } \\
\text { Tube HV } \\
\text { Tube Gain } \\
\end{array}$ & $\begin{array}{r}20 \\
2100 \mathrm{~V} \\
\approx 1 \mathrm{pC} \\
\end{array}$ & $\begin{array}{r}32 \\
1950 \mathrm{~V} \\
\approx 300 \mathrm{fC} \\
\end{array}$ \\
\hline $\mathrm{P} / \mathrm{T}$ Monitor & $\begin{array}{l}\text { \#Press. Sensors } \\
\text { \#Temp. Sensors }\end{array}$ & $\begin{array}{r}2 \\
2 \\
\end{array}$ & $\begin{array}{r}4 \\
68 \\
\end{array}$ \\
\hline HV Feedback & $\begin{array}{l}\text { Available ? } \\
\rho_{o}[\mathrm{mBar} / \mathrm{K}]\end{array}$ & $\begin{array}{l}\text { N/A } \\
3.338\end{array}$ & $\begin{array}{r}\text { Yes } \\
3.338\end{array}$ \\
\hline & $\begin{array}{l}S_{\text {pad }} \\
\alpha_{\text {pad }}\end{array}$ & $\begin{array}{c}\mathrm{N} / \mathrm{A} \\
119.1\end{array}$ & $\begin{array}{l}7.82 \pm 0.14 \\
120.8 \pm 0.7\end{array}$ \\
\hline & $\begin{array}{l}S_{B R D} \\
\alpha_{B R D}\end{array}$ & $\begin{array}{r}8.9 \\
113.0\end{array}$ & $\begin{array}{l}7.01 \pm 0.25 \\
112.7 \pm 0.7\end{array}$ \\
\hline
\end{tabular}


Both modifications were tested before starting the calibration run, and both resulted in significant noise reduction on the FHA. Consequently, the FHA quadrants at B0 were later modified in the same way. The results seen in the testbeam are summarized below.

Grounding Straps. The grounding of the FHA chambers is important in reducing ground loop noise in the FHA system. Ideally, the front and back FHA chamber skins (aluminum) should be electrically grounded together. Originally, this connection was made using copper tape which was wrapped around the side of the chamber. Unfortunately, the conductivity and adhesion of this tape has degraded significantly during the six years that these chambers have been in service. In a sample of 27 chambers, about $30 \%$ showed no ground connection between the chamber face panels. We remedied this problem by installing ground bus straps with reliable contact surfaces. We used a silver epoxy, with very small resistance, to glue brass pads to the chamber face panels. The pads glued to the front and back chamber skins were connected by a soldered copper braid. In addition, the midpoint of the braid was soldered to the electrical ground on the printed circuit board which extends from the edge of the chambers. Our test beam studies have shown that the noise, as measured by the standard deviation of pedestal distributions, is reduced from 35 counts to $\sim 10$ counts following the installation of these pads and ground straps.

We installed two grounding straps per chamber at B0 during the accelerator shutdown in February and March 1992. The resultant data from calibration (pedestal) runs and $\bar{p} p$ events show the coherent noise on pads to be greatly reduced.

Cables. The signals from FHA chamber cathodes (pads) are read out via a flat, daisy chain, ribbon cable. For the 1992-93 run, we must reduce the chamber gas gain to protect the chambers from glow mode at the expected higher luminosity. Thus, a corresponding increase in the amplifier gain of the front-end electronics is required. The new amplifier card has a small resistance to ground providing better noise suppression as well as a quasi-differential signal input. To better match the input to this new amplifier, the existing flat ribbon cables have been replaced with twisted-pair ribbon cables.

In order to test this new cable scherne, a set of twisted-pair cables was prepared for the testbeam FHA quadrant. We then observed the resulting effect on pedestals and crosstalk and compared these measurements with similar measurements obtained with flat ribbon cables. The test conclusions are summarized as follows: (a) the pedestal width improves using twisted-pair cables; (b) the cross-talk is reduced by $40 \%$ to a level less than $1 \%$ by using 
twisted-pair cables. Thus, all flat ribbon cables on the FHA at B0 were replaced (during the long shutdown in February 1992) with twisted-pair ribbon cables.

\section{Future Work}

Since the FHA is stably in operation, we started analyzing gas gain data and dijet data ( $E_{T}$ balancing studies). These analyses are essential to understand the energy scale of the gas calorimeters.

\section{Plug Upgrade Calorimetry}

Since we (Dr. Kamon and Mr. Wahl) joined the CDF effort to upgrade the plug electromagnetic and hadronic calorimeters (PEM, PHA), we are responsible for carrying out a detailed Monte Carlo simulation with a GEANT3 package for this new configuration. The geometry file for this detector simulation has been written [43]. The entire CDF calorimetry in 1995 is also ready.

The Monte Carlo study of the upgrade PEM and PHA is compared to the results obtained by the 1991 testbeam analysis of the prototype plug upgrade detector [3]. We report this work below.

\section{Electromagnetic Calorimeter}

The Monte Carlo calculation shows that the PEM calorimeter has a resolution of $\sigma / E$ $=(16.4 \pm 0.3 \%) / \sqrt{E} \oplus(0.6 \pm 0.2 \%)$ and has very good linearity. The testbeam results for resolution and linearity of the PEM are shown in Figures 37 and 38, respectively: an energy resolution of $15.7 \% / \sqrt{E}$ and very good linearity.

\section{Hadronic Calorimeter}

The hadronic calorimeter resolution is $\sigma / E=(88-2.8 \%) / \sqrt{E} \oplus(6.5 \pm 0.5 \%)$ from the Monte Carlo calculation. For the linearity, the Monte Carlo show a $22 \%$ and $13 \%$ drop in $E / p$ at 5 and $10 \mathrm{GeV} / \mathrm{c}$, respectively (Figure 40 ). The linearity at higher energies is good. 
Figure 40 shows the energy resolution plot from testbeam data. The testbeam data show an energy resolution of $78.7 \% / \sqrt{E}$. Figure 41 shows the testbeam results for linearity. The large drop in $E / p$ is also seen in the data. The $e / h$ ratio is 1.23 from the Monte Carlo. Testbeam results show $e / h$ to be $\approx 1.3$, in fair agreement with the Monte Carlo.

\section{Summary}

We found good agreement between the data and simulation. The performance of the new plug calorimeters is as good as that in the central calorimeters.

Since the geometry of the entire CDF calorimetry is ready, it is imporiant for us to carry out detailed physics simulation for the future collider run. We realize a.gicin tlirough this simulation work the poor performance of the tracking system in this region. Since our original motivation of working on the plug upgrade prcjec ${ }^{\dagger}$ was to siudy the required track. ing performance in this region, we proposicd a new tracking device for the SVX-II síage 2 disk system. The proposal is described in the following section.

\section{E. Gas Microstrip Chamber for SVX-II Stage 2}

Since the plug calorimeter will be replaced, a new tracking system in the plug region will be needed to increase the physics capability ( $b$ quark physics, $W$-asymmetry etc.) in the future physics runs (1995 and beyond): namely, to increase tr.e detection of lepton and other tracks from $b$ quark and $W$ decays.

Our group (Kamon, McIntyre and Trost) proposed a SVX-II disk system using the gas microsirip chamber technology to extend the b-physics capability [45]. We here summarize the proposal and its status in the CDF group.

\section{Introduction}

CDF is presently considering embarking on a two-stage upgrade to the silicon vertexing system (SVX) which would improve the vertexing performance for tracks in the central 
region, and then extend the solid angle coverage for this precision vertex detection to higher values of pseudorapidity. This SVX-II system [46] would consist, according to the plan already presented to the collaboration and to the PAC, of a stage- 1 barrel detector approximately doubling the length of the present SVX, followed in stage- 2 by the replacement of the forward portion of the VTX detector with a precision tracking detector consisting of a set of radial disks.

Stage 1 is intended to provide good precision over as much of the vertex region along the beam line in $z$ as possible, and so should be realized in a technology which will provide the best possible precision, i.e. silicon. The second portion of this upgrade (stage 2) is intended to improve the $\eta$ coverage of the detector for tracking, and so could be realized in a variety of technologies. We consider here a disk system for SVX-II stage 2 based on the use of gas microstrip (GM) detector technology.

\section{Physics and Performance Goals}

We envision this system to be mainly useful for improving the solid aısle acceptance for recognizing and locating rracks and for improving the precision with which these tracks car be extrapolated out to the calorimeter and in to locate the primary or possibly displaced vertices. Since jets 0 , even the decay products of $W$ 's, $B$ mesons and $\tau$ 's become more collimated in the forward region for a given $p_{T}$ as the total momentum goes up, it will be important to achieve good two-track separation capability in order to separate multiple charged particles within these jets.

Due to the fact that the radius of the the region inside the CTC is small $(r<27 \mathrm{~cm})$, the momentum resolution that is achievable within this region is also limited. It would be desirable, however, to be able to measure the sign of a given charged track up to a reasonably high value of particle $p_{T}$, for example the approximately $30 \mathrm{GeV} / \mathrm{c} p_{T}$ lepton resulting from the decay of a $W$ in the $W$-asymmetry measurement. We take as a physics goal the ability to measure the sign of the charge for a particle with $30 \mathrm{GeV} / \mathrm{c}$ transverse momentum up to a pseudorapidity value of approximately 3 units.

Benefits to other physics topics, such as the top mass measurement and $b$-physics topics, are also documented in the SVX-II proposal and the progress report to the PAC [46]. Figure 42 shows one conceptual configuration of the SVX-II (stages 1\&2) system taken from these documents. The geometrical parameters for barrels and disks studied by the SVX-II 
Table 9: Summary of barrel detector parameters [46]

\begin{tabular}{||c||c|c|c|c|c||}
\hline Element & $\begin{array}{c}\text { radius } \\
(\mathrm{cm})\end{array}$ & $\begin{array}{c}\frac{1}{2} \text { length } \\
(\mathrm{cm})\end{array}$ & $\begin{array}{c}\text { thickness } \\
(\mathrm{cm})\end{array}$ & $\begin{array}{c}\sigma_{r \phi} \\
(\mu \mathrm{m})\end{array}$ & $\begin{array}{c}\sigma_{z} \\
(\mu \mathrm{m})\end{array}$ \\
\hline Beam pipe & 2.60 & - & - & - & - \\
CTC (inner wall) & 27.6 & 155.0 & 0.10 & - & - \\
SVX II (stage 1) & $3.0,4.3,5.7,7.8$ & 51.0 & 0.03 & 12 & 30 \\
\hline
\end{tabular}

Table 10: Summary of radial disk (stage 2) parameters [46]

\begin{tabular}{||c|c||}
\hline$z(\mathrm{~cm})$ & $55.0,62.5,70.0,102.5,126.5,150.0$ \\
\hline Disk radius $(\mathrm{cm})$ & 27.0 \\
Thickness $(\mathrm{cm})$ & 0.03 \\
$\sigma_{r \phi}(\mu \mathrm{m})$ & 15.0 \\
$\sigma_{r}(\mu \mathrm{m})$ at $\eta=\infty$ & 30.0 \\
\hline
\end{tabular}

group are summarized in Tables 9 and 10. Although other similar geometries could probably be proposed, we concentrate here on the improvements to performance for $b$ physics noted by the group, and extracted here for reference.

Table 11 shows the results of the $B$ meson acceptances for $p_{T}(b)>10 \mathrm{GeV} / \mathrm{c}[46]$. The acceptance which requires both $B$ 's to decay semileptonically with $p_{T}(\ell)>2.5 \mathrm{GeV} / \mathrm{c}$ is also studied. Table 12 shows these results. The acceptance values for $B$-physics in this channel are significantly higher with SVX-II stage 2 than in the equivalent measurement with SVX-II stage 1.

The ability to tag the flavor of the $B$ meson is essential to all measurements involving mixing. As an example, we consider the measurement of a $\mathrm{CP}$ violating asymmetry in a neutral $B$ decay, $B \rightarrow \psi K_{s}$, where one must tag the flavor of the other $b(\rightarrow \ell \nu X)$ in the event. The cleanest signal will be the $B$ decay associated with $\psi \rightarrow \mu^{+} \mu^{-}$providing a clear trigger. The measurement of such an asymmetry presents an enormous experimental challenge because of (a) the small branching ratios involved and (b) the low $p_{T}$ of the events. Therefore, it is clear that high $\eta$ tracking (the disk system) will significantly improve the statistics of the measurement. 
Table 11: Geometric acceptance for all charged tracks from a $B$ meson in $p \bar{p}$ Collisions for $p_{T^{\prime}}(b)>$ $10 \mathrm{GeV} / \mathrm{c}[46]$

\begin{tabular}{|l||c|c||}
\hline Detector & $\begin{array}{c}\text { Single } B \\
\text { Accept.(\%) }\end{array}$ & $\begin{array}{c}\text { Both } B \\
\text { Accept.(\%) }\end{array}$ \\
\hline CTC & 52 & 31 \\
SVX & 23 & 10 \\
SVX-II stage 1 & 42 & 21 \\
SVX-II stage 2 & 64 & 45 \\
\hline
\end{tabular}

Table 12: Inclusive both $B$ acceptance for different pseudorapidity intervals of the dileptons for $p_{T}(\ell)>2.5 \mathrm{GeV} / \mathrm{c}$ and $p_{T}(b)>10 \mathrm{GeV} / \mathrm{c}[46]$

\begin{tabular}{||l||c|c|c||}
\hline Detector & $\begin{array}{c}|\eta(\ell \ell)|<1 \\
\text { Accept.(\%) }\end{array}$ & $\begin{array}{c}|\eta(\ell \ell)|<2 \\
\text { Accept.(\%) }\end{array}$ & $\begin{array}{c}|\eta(\ell \ell)|<3 \\
\text { Accept.(\%) }\end{array}$ \\
\hline CTC & 16 & 37 & 38 \\
SVX & 6.5 & 15 & 15 \\
SVX-II stage 1 & 12 & 28 & 29 \\
SVX-II stage 2 & 14 & 43 & 57 \\
\hline
\end{tabular}

In addition, the disk system should work well with the SVX-II barrel system. The data should be capable of being combined with the information from the barrel and/or CTC in time to be of use to form a trigger in the CDF data acquisition system. The ability to produce information useful in the SVT displaced-vertex trigger at Level 2, for instance, will be an important design criterion.

It will be important also to minimize the amount of material encountered by the particle between the interaction point and the calorimeters. The SVX-II barrel already will present approximately $20 \%$ of a radiation length to such a particle in the forward region due to its extended length [46]. This length, however, was chosen to give good vertex coverage for central particles produced in the extended luminous region along the beamline in $z$, and is not a tunable parameter. Thus, it is important that the rest of the tracking system in the forward direction present as little material as possible during the remaining path of the 
particle; so a geometry of disks is preferable to that of barrels in the forward direction.

Finally, the technology should be capable of withstanding several years of operation with the main injector at projected luminosities approaching $10^{32} \mathrm{~cm}^{-2} \mathrm{sec}^{-1}$, leading to a radiation dose at the inner radii of the disks projected to be a good fraction of a Megarad.

For the above reasons, the choice of technology for stage 2 of the upgrade is limited to gas microstrip or silicon disk detectors. In this report, we present a design of the disk chambers using gas microstrips to provide a detector and electronics readout capable of supporting a level 2 trigger even with a short (132 nsec) inter-bunch spacing and under high radiation levels.

\section{Gas Microstrip Chamber Technology}

Principle of Operation. The gas microstrip chamber was proposed some time ago $[47,48]$ in an attempt to reproduce the field structure of multiwire chambers on a much smaller scale. It consists essentially of a sequence of alternating thin conductive anode and cathode strips placed on an insulating substrate. A drift cathode defines a gas region for collection of ionization charges, while a conductive plane on the back side of the substrate ("backplane") provides an additional tool for field shaping. Application of suitable voltages to the four electrodes creates a proportional gas multiplication field near the anodes.

Under favorable circumstances, a signal is also induced on the backplane by capacitive coupling. Dividing the backplane up into strips, e.g. at right angles to the anodes and cathodes, allows one to read out information on a second dimension from the same detector. A 1." $\times 1$ " GM detector with two-dimensional readout was proposed and realized by the group of R. Bellazzini [49]. Figure 43 shows the cross sectional view of his detector and its field lines. This chamber design will be taken as a basis for our proposed SVX-II stage 2 disk system.

In order to avoid or control charge-up effects on the surface, the substrate cannot be a near-ideal insulator. From experience [50], its surface resistivity should be about $10^{13} \Omega / \square$ to within an order of magnitude. Some substrate materials that do not have such a resistivity may be tuned to it using boron or argon ion-implantation. The implanted layer plays an important role in how large signals can be induced on the backplane. For this reason, the substrate in the above conceptual design needs to be thin. 
Demonstrated Performance. A successful demonstrations of working GM detectors has been produced by Bellazzini's group [49]. In his detector, a quartz plate is used as a mechanical support. The support layer has $0.2 \mu \mathrm{m}$ thick titanium backplane, an $\mathrm{SiO}_{2}$ substrate layer of $1.8 \mu \mathrm{m}$ thickness, and $2 \mu \mathrm{m}$ aluminum anodes/cathode strips. The $\mathrm{SiO}_{2}$ layer is ionimplanted at the surface with a dose of $5 \times 10^{16}$ boron ions $/ \mathrm{cm}^{2}$. The drift volume is $3 \mathrm{~mm}$ thick and filled with an Ar-DME (Di-Methyl-Ether) mixture in a 90:10 ratio.

The detector can sustain rates as high as $10^{7}$ particles $/\left(\mathrm{mm}^{2} \cdot \mathrm{sec}\right)$, and shows no charge-up effects over 3 days of $10^{4}$ particles $/\left(\mathrm{mm}^{2} \cdot \mathrm{sec}\right)$. However, a gain loss by $15 \%$ was measured due to aging after collecting 0.016 Coulomb $/ \mathrm{cm}$ charge. This is estimated to be equivalent to over six years running at $50 \mathrm{~cm}$ away from the beam at the LHC.

The only severe problem is the sizeable detector capacitance for the backplane strips $(1-2 \mathrm{pF} / \mathrm{cm})$ which is not adequate to cheaper, low-power CMOS electronics.

\section{Design - An Overview}

The design goal is to provide a good pattern recognition capability (for the CTC inner layers and upgraded plug calorimeter) with sufficient layers within the available space.

The optimization of the gas microstrip chambers should be made at the following points at the Teiatrnn operation and CDF b-physics:

- Short charge collection time ( $<132$ nsec bunch period);

- Operation in a flux of $10^{4}$ particles $/\left(\mathrm{mm}^{2} \cdot \mathrm{sec}\right)^{3}$;

- Radiation resistance to the level of $1 \mathrm{Mrad}$;

- Accuracy $(\approx 50 \mu \mathrm{m})$ and double track separation $(\approx 200 \mu \mathrm{m})$;

- Small amount of material: $3-4 \%$ of a radiation length ${ }^{4}$ and little aging at $2 \mathrm{fb}^{-1}$.

Since the time until Run II (November 1995) is fairly short compared to the production time to be expected, we have decided to use Bellazzini's design as closely as possible for the SVX-II disks. We, however, will improve the detector performance with a new backplane strip design:

\footnotetext{
${ }^{3} F=\sigma_{\text {inel }} \mathcal{L}\left(d N_{c h} / d \eta\right) /\left(2 \pi z^{2} \tan ^{2} \theta\right)$, where $\theta$ is a polar angle with respect to the beam pipe, $z$ is the position of disk. Typical numbers of $\bar{p} p$ collisions at Tevatron are $\sigma_{\text {inel }}=59 \mathrm{mb}, \mathcal{L}=10^{32} \mathrm{~cm}^{-2} \mathrm{sec}^{-1}$, and $d N_{c h} / d \eta=4.1$.

${ }^{4}$ In a typical organic material, a relativistic charged particle flux of $3 \times 10^{13} \mathrm{~cm}^{-2}$ produces an ionizing dose of 1 Mrads.
} 
1. The new design of backplane strip pattern substantially reduces the detector capacitance so that we can use cheap CMOS-based electronics.

2. Titanium-gold-titanium sandwich film instead of a single titanium film allows us to use the low-pressure version of the chemical vapor deposition (CVD) for growing an $\mathrm{SiO}_{2}$ substrate layer, which is much less expensive than the plasma-enhanced version.

One can design a two-dimensional readout GM detector with modifications of Bellazzini's chamber to accommodate a disk ring geometry:

(a) Backplane electrode strips made of thin $\mathrm{Ti}-\mathrm{Au}$ - $\mathrm{Ti}$ sandwich films on a mechanical support layer (e.g. $300 \mu \mathrm{m}$ thick silicon wafer) to measure the azimuthal angle $\phi$ :

- Truly radial pattern (keystoned strips) to cover a ring section ( $7 \mathrm{~cm} \mathrm{long}$ );

- Strip width of $100 \mu \mathrm{m}$ at $r=25 \mathrm{~cm}$, giving $r \cdot \sigma_{\phi}=29 \mu \mathrm{m}$;

- Small detector capacitance $(\approx 5 \mathrm{pF})$;

- Almost the same pulse height for the induced signals as on the anodes;

- The same amplifiers as used on the anodes.

(b) A very thin $(\approx 1.8 \mu \mathrm{m})$ substrate (ion-implanted $\left.\mathrm{SiO}_{2}\right)$ on the backplane strips.

(c) "MWPC" using high-definition photolithography on the thin substrate to measure the radial coordinate $r$ :

- Alternating anode ( $5 \mu \mathrm{m}$ wide) and cathode ( $70 \mu \mathrm{m}$ wide) strips (e.g. aluminum);

- Anode-to-anode pitch of $200 \mu \mathrm{m}$ to give $\sigma_{r}=58 \mu \mathrm{m}$;

- "Pie"-shaped modules;

- Gas: Ar plus quencher (drift time of about $100 \mathrm{nsec}$ in $5 \mathrm{~mm}$ gas gap);

- Gas gain of 2000-5000 allowing the use of cheap CMOS electronics.

Thus, one layer of GM detectors can provide two-dimensional information $(r-\phi)$ within a. depth of less than $6 \mathrm{~mm}$ ( $5 \mathrm{~mm}$ for gas gap and $<1 \mathrm{~mm}$ for solid material) and with reasonably low density.

We divide the ring disks into $30^{\circ}$ sections consisting of four tile chambers, labeled as types A, B, C1 and C2 (see Figure 44 and Table 13). The sizes are chosen to fit inside a $15 \times 15 \mathrm{~cm}^{2}$ (or 6 " $\times 6$ ") for which metalization, mask production, and aligner machines for photolithography are commercially available. The two dimensions of the readout will be 
Table 13: Summary of tile geometry and strip patterns

\begin{tabular}{|c|c|c|c|c|c|c|c|}
\hline \multirow{2}{*}{$\begin{array}{l}\text { Tile } \\
\text { Type }\end{array}$} & \multirow{2}{*}{$\begin{array}{c}\text { Rough Size }\left(\mathrm{cm}^{2}\right) \\
r_{\max } \phi \times\left(r_{\max }-r_{\min }\right)\end{array}$} & \multirow[t]{2}{*}{$\phi$ range } & \multicolumn{2}{|c|}{$r$ range $(\mathrm{cm})$} & \multirow{2}{*}{$\begin{array}{c}\Delta \phi \\
(\mu \mathrm{rad}) \\
\end{array}$} & \multirow{2}{*}{$\begin{array}{c}r \times \Delta \phi \\
(\mu \mathrm{m})\end{array}$} & \multirow{2}{*}{$\begin{array}{l}\text { \#strips } \\
\text { (radial) }\end{array}$} \\
\hline & & & $r_{\min }$ & $r_{\max }$ & & & \\
\hline $\mathrm{A}$ & $5.8 \mathrm{~cm} \times 7 \mathrm{~cm}$ & $30^{\circ}$ & 4 & 11 & 800 & $32-88$ & 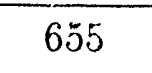 \\
\hline B & $9.4 \mathrm{~cm} \times 7 \mathrm{~cm}$ & $30^{\circ}$ & 11 & 18 & 400 & $44-72$ & 1310 \\
\hline $\mathrm{C} 1, \mathrm{C} 2$ & $6.5 \mathrm{~cm} \times 7 \mathrm{~cm}$ & $15^{\circ}$ & 18 & 25 & 400 & $72-100$ & 655 \\
\hline
\end{tabular}

provided by keystoned radial strips on the backplane for $\phi$-measurement (Figure 45) and by anode and cathode strips to measure the radius $r$ (Figure 46). The width of backplane strips is $100 \mu \mathrm{m}$ maximum, while the anode-to-anode pitch is a constant $200 \mu \mathrm{m}$. The elements on each submodule are completed by preamplifiers, edge connectors and common highvoltage busses for each group of lines (anodes, cathodes, backplane lines). Figure 47 shows a schematic drawing of one tile with its local readout electronics.

In a variation for providing a useful trigger at level 2 , the backplane layer for some disks may have a pad pattern rather than keystoned strips (see Figure 48). These can be made large enough not to increase the channel count. Furthermore, we can reduce the number of tiles per $30^{\circ}$ sector to two. The size of tile is still within the $6 " \times 6$ " area. This option is under consideration.

\section{Substrate}

Silicon dioxide is quite a good insulator. Its surface resistivity is larger than $10^{17} \Omega / \square[51]$. The ion implantation serves to reduce this value to a more acceptable range. The surface resistivity has been measured on one of Bellazzini's chamber to be about $10^{16} \Omega / \square$, and the volume resistivity to be $10^{11} \Omega \mathrm{cm}$. The surface resistivity is still fairly high but it corresponds, due to the small thickness of the substrate, to about the same volume resistivity of other substrates that have been used with thicknesses of $100 \mu \mathrm{m}$ or more.

An explanation for the (measured) absence of charge-up effects may be as follows: in Bellazzini's operating conditions, the electric field lines that do not connect the anodes to the drift cathode but stay near the substrate are forced to bend into the substrate rather than connecting anodes and cathodes along the surface. Thus the ions released in the signal avalanche at the anodes are guided into the ion-implantation layer rather than near the sur- 
face to the cathode strips. This path is much shorter than the one to the cathodes and hits a conductive layer, i.e. the implanted one, to collect and transport the charges away. On the surface, the transport speed would be too small to evade an accumulation of charges. Actually, they obtained a small amount of charge on the cathode strips [49]. This is the contrast to our measurement with unimplanted Upilex chamber ${ }^{5}: 80 \%$ pulse heights relative to the anode signal on the cathode strips and $65 \%$ pulse heights on the (unsegmented) back plane [52].

\section{Thicknesses}

The total thickness is dominated (about $95 \%$ ) by the silicon wafer as a mechanical support. These can be obtained with $300 \mu \mathrm{m}$ thickness, corresponding to $0.31 \%$ of a radiation length. A total of 6 chambers thus gives $1.9 \%$ and, where chambers in successive radial ranges are overlapped to avoid dead regions, about $4 \%$. This is well within acceptable limits for keeping multiple scattering and photon conversion under control.

\section{Chemical Vapor Deposition (CVD)}

This process is relevant for growing a thin substrate layer of $1.8 \mu \mathrm{m}$ thick $\mathrm{SiO}_{2}$ onto a metal. There are three variations of this procedure: (a) at atmospheric pressure, (b) at low pressure (or "vacuum") of the order of $10^{-3}$ Torr, and (c) "plasma enhanced". The choice is governed by the materials involved. The low pressure version is good enough for deposition of $\mathrm{SiO}_{2}$ onto titanium, while requiring the use of the plasma-enhanced version on aluminum to obtain enough density and adhesion of the deposited $\mathrm{SiO}_{2}$ on the metal.

We consider a titanium-gold-titanium sandwich instead of aluminum. The benefit of changing the single titanium layer to a titanium-gold-titanium sandwich layer would be to use the low-pressure CVD which is much less expensive than the plasma-enhanced version.

\section{Metal Coating}

We expect a severe problem with Bellazzini's chamber when the size of 1 " $\times 1$ " is scaled up to near $4 " \times 4 "$ [53]. The very thin titanium backplane $(0.2 \mu \mathrm{m})$ owes its choice to

\footnotetext{
${ }^{5}$ Upilex substrate is 70 times thicker than the silicon dioxide layer of Bellazzini's chamber. The anode-cathode gap is less than half of his [51].
} 
the adhesion properties of quartz surfaces. However, titanium has a fairly large resistivity. Longer lines would lead to a position dependence of pulse height. A solution [53] is to use a silicon wafer of a suitable size instead of the quartz, and thermally oxidize the surface. Then the backplane metal can be aluminum with a suggested thickness of $2 \mu \mathrm{m}$. A further consequence is that for the CVD process one has to use the plasma enhanced version rather than the low pressure version. This is again connected to adhesion properties and to formation properties of the $\mathrm{SiO}_{2}$ layer.

An alternative option is to replace the single aluminum or titanium layer by a titanium-gold-titanium sandwich to keep the particularly good adhesion of the titanium available and get around the relatively high resistivity of the titanium as a conductor.

The ion implantation process and the metal for the anode and cathode strips (aluminum) remain unaffected.

\section{Lithography and Etching Techniques}

Photolithography and etching are the tools to engrave anode and cathode strips in a large metal area. The size of this area (and thereby the chamber) will be limited by the production equipment available. For CDF, we are looking at sizes up to 6 " $\times 6$ ". The smallest width of strip is $5 \mu \mathrm{m}$ which cannot reliably be accomplished with PC-board class machines. Thus we will need a more expensive machine, made for integrated circuit work, like the MA- 6 exposure from Karl Suss which just covers the required field size for our application [54].

Once the photoresist has been deposited on the metal layer and exposed with the desired pattern, this pattern has to be etched into the metal. Two types of etching processes can be used. The dry (plasma) etching process is preferable over the wet (chemical) process because it delivers better uniformity of the strip width. We are in the process of installing a dry etching facility. Therefore, we can evaluate both processes.

\section{Masks}

Masks are pieces of glass with the line pattern printed on them that are to be transferred by photolithography onto the metal layers of the chambers as just described. The line pattern itself is predetermined by CAD programs and transferred onto a chrome layer on the mask. For the SVX-II disks, we need at least two different masks: for the anode and 
cathode lines (with their electronics and HV supplies) and for the backplane strips. From ring to ring, these patterns vary adapting to the particular geometrical location.

Because of the long $i$, eraction region compared to the distance of the disks away from it, a projective geometry layout as pursued by SDC [55], is useless for triggering. In addition, we are thinking about plugging into the trigger level 2 rather than level 1 in the case of SDC. Thus the following idea appears attractive: in some chambers, do not use strips all the way through on the backplane to measure $\phi$ for determining a transverse momentum and rely on the radial tile center as a sufficient measurement of $r$ but put "crude" pads of sizes of the order of $1 \mathrm{~cm}^{2}$ onto the backplanes of, say, three chambers (Figure 48). Then a useful measurement of $r$ or equivalently $\theta$ can be made without problems and without requiring the longitudinal beam constraint. This implies a third type of mask for the pad pattern. In fact, Bellazzini's group has also reported on such a chamber [49]. The mask sizes we need for CDF for $6 " \times 6$ " area photolithography are common in the industry.

\section{Detector Capacitance}

The small thickness of the substrate is required to observe signals on the backplane of about the same pulse height as on the anodes. Due to the conductive ion-implanted layer near the anodes and cathodes, the backplane is shielded from pickup of signals if the substrate thickness is as large as, for example, the ones we have used in our existing test chambers. The thickness of the silicon dioxide in Bellazzini's chamber leads to a sizeable capacitance per line in our CDF geometry. The typical size of the backplane strip is $7 \mathrm{~cm}$ long $\times 86 \mu \mathrm{m}$ wide. Ignoring any possible effect from a floating implantation layer and looking at just one backplane strip area overlapping with anode and cathode strips, we find $C=$ $\kappa \epsilon_{0}\left(A_{\text {anode }}+A_{\text {cathode }}\right) / d=35 \mathrm{pF}$, with $A_{\text {anode }}=7 \mathrm{~cm} \times 86 \mu \mathrm{m} \times 5 \mu \mathrm{m} / 200 \mu \mathrm{m}, A_{\text {cathode }}=$ $7 \mathrm{~cm} \times 86 \mu \mathrm{m} \times 70 \mu \mathrm{m} / 200 \mu \mathrm{m}$, the dielectric constant $\kappa=4$ and $d=1.8 \mu \mathrm{m}$ for the $\mathrm{SiO}_{2}$ substrate layer. ${ }^{6}$ This capacitance can be reduced by a new design of the backplane strips as shown in Figures $49 \mathrm{a}$ and $49 \mathrm{~b}$. In this design, the overlapped area between the cathode strip and backplane strips is reduced by a factor of 14 . Thus,

$$
C=4.7 \mathrm{pF} \text {. }
$$

We could reduce this value by another factor of 1.7 by increasing the thickness of the substrate to $3 \mu \mathrm{m}$. Therefore, $C=2.8 \mathrm{pF}$. We must investigate the possibility of increasing the

\footnotetext{
${ }^{6}$ This corresponds to $5 \mathrm{pF} / \mathrm{cm}$ and bigger than the number $(2 \mathrm{pF} / \mathrm{cm})$ from Bellazzini's chamber.
} 
Table 14: Summary of gas gap range

\begin{tabular}{||l|l||l||}
\hline \multicolumn{2}{|c||}{ Requirement } & Gap range \\
\hline Charge collection time: $t=d / v_{d r i f t}$ & $\leq 132 \mathrm{nsec}$ & $\leq 6.6 \mathrm{~mm}$ \\
Efficiency: $\epsilon=1-\exp \left(-n_{p} \cdot d\right)$ & $\geq 99 \%$ & $\geq 1.5 \mathrm{~mm}$ \\
Double track separation: $\Delta x=\sigma_{T}$ & $\leq 200 \mu \mathrm{m}$ & $\leq 10 \mathrm{~mm}$ \\
\hline
\end{tabular}

thickness of the substrate to $3 \mu \mathrm{m}$ and study the resulting pulse height on the backplane.

Gas

Since Bellazzini's group demonstrated the performance of the GM chamber, we can use the same gas: Ar-DME (90:10). DME is a good quencher to prevent discharges, either spontaneous or from induced (UV light from avalanche) cathode emission. We should note that judgements from experience with regular wire chambers cannot simply be transferred: (a) the achievable gains are less in GM detectors than in wire chambers by abo:t an order of magnitude, and also their aging behavior is different (see below); (b) a further difference is the shape of the electric field in the avalanche region - the rise begins with a shape proportional to $r^{-4}$ at the anode and changes over to the $r^{-1}$ form one finds all the way through the avalanche region in wire chambers [56]. While this might favor higher signals in GMC's, the absolute field strength cannot be kept as high due to the much closer distances from the anodes to the shaping cathodes giving a higher probability for high-voltage breakdown.

The gas properties are characterized using

$$
\begin{aligned}
n_{p} & =\text { number of primary ion-pairs } / \mathrm{cm} \\
n_{\text {tot }} & =\text { total ionization ion-pairs } / \mathrm{cm}, \\
v_{d r i f t} & =\text { drift velocity }(\mathrm{cm} / \mu \mathrm{sec}) \\
\sigma_{T} & =\text { transverse diffusion }(\mu \mathrm{m})
\end{aligned}
$$

We take $n_{p}=30, n_{\text {tot }}=100, v_{\text {drift }}=5 \mathrm{~cm} / \mu \mathrm{sec}, \sigma_{T}=200 \mu \mathrm{m}$ (for $\left.d=1 \mathrm{~cm}\right)$. Table 14 shows the range of gas gap in the condition given above. The gas gap we suggest from the table is $5 \mathrm{~mm}$. 


\section{Operating Voltage}

Bellazzini'z detector was operating at the following voltages (for pixels) [49]:

$\begin{array}{llr}\text { Drift voltage } & V_{D}=-1500 \mathrm{~V} \\ \text { Anode voltage } & V_{A}=+310 \mathrm{~V} \\ \text { Cathode voltage } & V_{C}= & 0 \mathrm{~V} \\ \text { Backplane voltage } & V_{B}= & 0 \mathrm{~V}\end{array}$

It should be noted that the best working conditions have been reported when applying the same potential on the cathodes and backplanes: $V_{C}=V_{B}$.

In the geometry of Bellazzini's chamber, the distance between the backplane and anodes is much shorter than that between the anode and cathode. With these voltages, therefore, the field lines are forced into the drift electrode or the substrate where they immediately reach the ion-implanted (fairly conductive) layer (see Figure 43). The ions out of the avalanche clouds above the anode are thereby guided onto the layer and discharged on it rather than on the cathode strip. This would explain why the surface of Bellazzini's chambers simply does not charge up even during his highest rate tests. Another effect of this configuration is that the backplane receives a strong induced signal of about the same pulse height as the anode (at Bellazzini's substrate thickness) while the shaper cathodes would not see a reasonably recognizable signal at all from minimum ionizing particles.

\section{Aging}

In wire chambers, combinations of gases and materials allow to collect $\approx 1 \mathrm{C} / \mathrm{cm}$ on a wire without aging. Assuming the same intensity of incident flux, a microstrip chamber divides the same charge over about ten anodes. The actual observation is much different: Bellazzini's detector shows a $15 \%$ degradation after $16 \mathrm{mC} / \mathrm{cm}$ on the chamber [49]; it is operated with Ar-DME (90:10). The total charge of $16 \mathrm{mC} / \mathrm{cm}$ corresponds to 6 years of operation at LHC $50 \mathrm{~cm}$ away from the beam axis at design luminosity $\left(10^{34} \mathrm{~cm}^{-2} \mathrm{sec}^{-1}\right)$.

In order to estimate the aging limit at $F=10^{6}$ particles $/\left(\mathrm{cm}^{2} \mathrm{sec}\right)$ at $\mathcal{L}=10^{32}$ $\mathrm{cm}^{-2} \mathrm{sec}^{-1}$ (Tevatron), we take the parameters for a microstrip chamber to be $N_{\text {anode }}$ (the number of anode strips per $\mathrm{cm})=50, d$ (gas gap) $=5 \mathrm{~mm}, G=2 \times 10^{3}, n_{\text {tot }}=100$ ionpairs $/ \mathrm{cm}$. The number of electrons $\left(N_{e}\right)$ on a anode strip will be $10^{5}$. A total charge collected 
Table 15: Summary of channel counts

\begin{tabular}{||l||c|c|l||}
\hline \multicolumn{1}{|c|}{ Measurement } & $\begin{array}{c}\text { per } 30^{\circ} \text { module } \\
(4 \text { tiles })\end{array}$ & $\begin{array}{c}\text { per layer } \\
(12 \text { modules })\end{array}$ & $\begin{array}{c}\text { per SVX II } \\
(12 \text { layers }=576 \text { tiles })\end{array}$ \\
\hline$r(4 \mathrm{~cm}<r<25 \mathrm{~cm})$ & 1400 channels & $16.8 \mathrm{~K}$ channels & $201.6 \mathrm{~K}$ channels \\
$\phi$ & 3275 channels & $39.3 \mathrm{~K}$ channels & $471.6 \mathrm{~K}$ channels \\
\hline
\end{tabular}

in one year will be

$$
\begin{aligned}
Q & =e(\mathrm{C}) \times N_{e} \times F\left(\mathrm{~cm}^{-2} \mathrm{sec}^{-1}\right) \times T(\mathrm{sec}) \times \frac{1}{N_{\text {anode }}} \\
& =\left(1.6 \times 10^{-19}\right) \times 10^{5} \times 10^{6} \times 10^{7} \times \frac{1}{50} \\
& =3.2\left(\mathrm{mC} \cdot \mathrm{cm}^{-1} \cdot \mathrm{yr}^{-1}\right) .
\end{aligned}
$$

If the limit is set to $16 \mathrm{~m} \mathrm{C} / \mathrm{cm}$, the aging limit for CDF is about 5 years and is not expected to be a serious problem.

\section{Channel Counts}

Total number of channels of anode ( $r$-measurement) and backplane ( $\phi$-measurement) strips is $673 \mathrm{~K}$ as summarized in Table 15.

\section{Front-end Electronics}

In most high energy hadron collider experiments one needs low noise, low power and radiation-hard electronics designed with suitable pipelining and rapid digitization capable of supporting a trigger processor. In the present case a trigger is of interest at level 2 , after some $5 \mu \mathrm{sec}$. In particular, the SVX-II group is looking for [46]:

- Good signal to noise which should not degrade after one $\mathrm{fb}^{-1}$ to worse than $12: 1$.

- Radiation hardened to the level of 1.0 Mrad which corresponds to an integrated luminosity in excess of $2 \mathrm{fb}^{-1}$

- Risetime and shaping time compatible with Main Injector luminosity of the order $10^{32}$ $\mathrm{cm}^{-2} \mathrm{sec}^{-1}$ and bunch separation as short as $132 \mathrm{nsec}$. 
- Multi-event pipelining of $1.2 \mu \mathrm{sec}$ duration of the level 1 trigger.

- Rapid sparsification, digitization and readout of data $(<5 \mu \mathrm{sec})$ for use in level 2 trigger processors.

- 128 channels/chip:

- Power consumption/channel $(<4 \mathrm{~mW})$ constraints imposed by mechanical and cooling design limits

- 250 nsec peaking time

- Detector capacitance: $20 \mathrm{pF}$ at $\mathrm{AC}$ coupled

- A minimum number of control signals involving high speed clocks which can produce noise in other parts of the detector. The exception would be fiber optic control signals.

We should test the readout of a gas microstrip chamber using the SVX-II chips ${ }^{7}$.

\section{Simulation}

The measurement of particle momentum to be made in the disks is from the $\phi$ trajectory of each particle: two $\phi$ measurements at two locations $\left(r_{1}, z_{1}\right)$ and $\left(r_{2}, z_{2}\right)$.

$$
\phi_{2}-\phi_{1}=\frac{\left(z_{2}-z_{1}\right) \tan \theta}{2 \rho}
$$

where

$$
\begin{aligned}
\rho(\mathrm{m}) & =\frac{p_{T}(\mathrm{GeV} / \mathrm{c})}{0.3 B(\text { tesla })} \\
\phi & =\phi_{0}+\frac{r}{2 \rho} .
\end{aligned}
$$

The accuracy of $p_{T}$ measurement is expressed as

$$
\frac{\sigma_{p_{T}}}{p_{T}^{2}}=\frac{2 \sqrt{2} \sigma_{\phi}}{0.3 B \Delta z \tan \theta}
$$

\footnotetext{
${ }^{7}$ For the readout test at Texas A\&M, we are using QPA02 chips [57] containing only four channels. They are useful for us because some of their other parameters are fairly competitive: the amplifier gain is $17 \mathrm{mV} / \mathrm{fC}$; the shaping time is $14 \pm 2 \mathrm{nsec}$ FWHM. The total power consumption per channel is $42 \mathrm{~mW}$ higher than is acceptable for dense environments such as the SVX II.
} 
Table 16: Summary of radial disk position and $\eta$ coverage. $\eta>3.5$ is not considered.

\begin{tabular}{|c|c|c|c|c|}
\hline \multirow[b]{2}{*}{ System } & \multirow[b]{2}{*}{$z(\mathrm{~cm})$} & \multicolumn{3}{|c|}{$\eta$-coverage } \\
\hline & & $z_{v t x}=0 \mathrm{~cm}$ & $z_{v t x}=30 \mathrm{~cm}$ & $z_{v t x}=60 \mathrm{~cm}$ \\
\hline Barrel & - & $0.0-2.5$ & $0.0-1.7$ & - \\
\hline Disk 1 & 60.0 & $1.6-3.5$ & $1.3-2.7$ & - \\
\hline Disk 2 & 70.0 & $1.8-3.5$ & $1.4-3.0$ & $\mathrm{xxx}-1.7$ \\
\hline Disk 3 & 80.0 & $1.9-3.5$ & $1.5-3.2$ & $\mathrm{xxx}-2.3$ \\
\hline Disk 4 & 110.0 & $2.2-3.5$ & $1.9-3.5$ & $1.5-3.1$ \\
\hline Disk 5 & 130.0 & $2.4-3.5$ & $2.1-3.5$ & $1.8-3.5$ \\
\hline Disk 6 & 150.0 & $2.5-3.5$ & $2.3-3.5$ & $2.0-3.5$ \\
\hline
\end{tabular}

where $\Delta z=z_{2}-z_{1}$. In our disk design, $\Delta \phi$ pitch $\left(\sigma_{\phi}\right)$ is constant. Since the distribution of the $z$-vertex has a $\sigma_{z}$ of $30 \mathrm{~cm}$, the location of the first disk is assumed to be at $z=60 \mathrm{~cm}$ $\left(2 \times \sigma_{z}\right)$ instead of $55 \mathrm{~cm}$ in Ref.[46]. Table 16 shows the (tentative) positions of 6 disks and its $\eta$ coverage of each disk at various vertex positions. The disks become important to cover a large $\eta$ region when the vertex is more than $30 \mathrm{~cm}$ off from the detector center.

Momentum resolution is calculated using the above formula in cases of two-disk combinations $^{8}$ (1\&3 or $4 \& 6$ ). Table 17 shows the results with $\sigma_{\phi}=\Delta \phi / \sqrt{12}$, where $\Delta \phi$ $=400 \mu \mathrm{rad}$. For a track of $p_{T}=10 \mathrm{GeV} / \mathrm{c}$, the resolution $\delta p_{T} / p_{T}$ will be $10 \%$ to $20 \%$ depending on $\eta$. It should be noted that we finished writing the geometry file for the CDF tracking system (CTC, SVX-II) using GEANT3 [58]. Therefore, we will start a detailed physics simulation to evaluate the tracking performance.

\section{Schedule}

We plan to have small test chambers with size of $2 " \times 2$ " by the beginning of 1993 . A full-size prototype chamber (up to $6^{\prime \prime} \times 6^{\prime \prime}$ ) needs to be finished and demonstrated to operate to specification before the end of FY93.

For small test chambers, we can use facilities in another laboratory on the campus, combined with some steps done outside (CVD, ion-implantation). For full-size chambers for

${ }^{8}$ Disks 2 and 5 might be pad chamber as shown in Figure 48. 
Table 17: Momentum resolution determined by iwo disks ( $1 \& 3$ or $4 \& 6)$ with $\sigma_{\phi}=115 \mu \mathrm{m} . \eta>3$ is not considered.

\begin{tabular}{||c||c|c||c|c||c|c||}
\hline \multicolumn{1}{||c||}{} & \multicolumn{2}{c||}{$z_{v t x}=0 \mathrm{~cm}$} & \multicolumn{2}{c||}{$z_{v t x}=30 \mathrm{~cm}$} & \multicolumn{2}{c||}{$z_{v t x}=60 \mathrm{~cm}$} \\
\hline Disks & $\eta$-coverage & $\begin{array}{c}\delta p_{T} / p_{T}^{2} \\
\left(\times 10^{-2}\right)\end{array}$ & $\eta$-coverage & $\begin{array}{c}\delta p_{T} / p_{T}^{2} \\
\left(\times 10^{-2}\right)\end{array}$ & $\eta$-coverage & $\begin{array}{c}\delta p_{T} / p_{T}^{2} \\
\left(\times 10^{-2}\right)\end{array}$ \\
\hline $1 \& 3$ & $2.0-2.5$ & $1.40-2.34$ & $1.5-2.3$ & $0.82-1.91$ & - & - \\
$4 \& 6$ & $2.5-3.0$ & $1.17-1.93$ & $2.3-3.0$ & $0.95-1.93$ & $2.0-3.0$ & $0.70-1.93$ \\
\hline
\end{tabular}

the SVX II, a lithography machine would need to be purchased. An appropriate machine ${ }^{9}$ is the model MA-6 from Karl Suss [54] at a price of about $\$ 180 \mathrm{~K}$. Also, if the corresponding design variant will be the one of choice, a machine for low-pressure or plasma-enhanced CVD would be needed.

\section{Summary}

We have described a possible design of the GMC tracking system for the CDF SVX-II stage 2 [46]. It should be noted that we have a new design of the backplane strips which will substantially reduce the detector capacitance. The summary of parameters is given in Table 18. The discussion relies on many existing elements: the design of the working chamber by R.Bellazzini's group [49], experience with larger-size chambers by NIKHEF group [56], electronics developments not only specific for these chambers but also for silicon strip detectors, the ungoing research and development activities within the SDC collaboration [55] and the experience and skills of our group [51].

The required scale-up from Bellazzini's chamber is modest. The current development tasks refer more to details rather than principle problems. The system offers high speed, good precision, fairly easy handling (e.g. alignment, compared to silicon strips) and a very adaptable/flexible readout geometry. The proposed GM detector is known to be robust with respect to charge-up problems and radiation sensitivity. It is expected to survive for five years or more at $10^{32} \mathrm{~cm}^{-2} \mathrm{sec}^{-1}$. The disk system nearly doubles the acceptance for the $b$ quarks compared to the case of only the barrel detector. Therefore, it plays an important

\footnotetext{
${ }^{9}$ We have searched for others (e.g. OAI) and checked the machine quality by interviewing customers who are using the machines. Those are less expensive, but are not adequate performance and precision.
} 
Table 18: Summary of GMC disk (stage 2) parameters

\begin{tabular}{||c|c||}
\hline \multicolumn{2}{|c||}{3 rings, 4 tiles per $30^{\circ}$ sector } \\
\hline$z_{\text {disk }}(\mathrm{cm})$ & $60.0,70.0,80.0,110.0,130.0,150.0$ \\
\hline Total number of channels & $673 \mathrm{~K}$ \\
Disk radius & $4 \mathrm{~cm}-11 \mathrm{~cm}, 11 \mathrm{~cm}-18 \mathrm{~cm}, 18 \mathrm{~cm}-25 \mathrm{~cm}$ \\
Thickness $/$ disk & $300 \mu \mathrm{m}$ of silicon or glass \\
$\sigma_{r \phi}($ at $r=25 \mathrm{~cm})$ & $29 \mu \mathrm{m}$ \\
$\sigma_{r}($ at $\eta=\infty)$ & $58 \mu \mathrm{m}$ \\
Gas gap & $5 \mathrm{~mm}$ \\
Gas gain & $>2000$ \\
Anode/backplane signals & $>10^{5}$ electrons \\
\hline
\end{tabular}

role in b-physics at CDF.

\section{F. Future Plans and CDF Upgrade Participation}

\section{CDF Physics Analysis}

A central goal of the present physics run of CDF is the search for the top quark. We now know that top lies above the $W$ mass, so that it will decay via $t \rightarrow W+b$. The key challenge then is to identify events of this kind among the total ensemble of $W$ events. We have developed a technique of partial reconstruction of $B$ mesons with Monte Carlo events through the following decay modes:

$$
\begin{array}{ll}
B \rightarrow D^{*}+\rho & (\approx 2 \%) \\
B \rightarrow D^{*}+\ell \nu & (\approx 10 \%)
\end{array}
$$

Profs. Kamon and McIntyre started looking at the actual data sample to carry out the above physics program with the assistance of Drs. Scarpine and Wolinski and Mr. Done.

Drs. Kamon and Wolinski also work on the search for the lightest $b$-baryon $\left(\Lambda_{b}\right)$ with Messrs. Kokkoris and Lu. This analysis will be done by using the CTC $d E / d x$ measurement 
to separate $\pi$ and $p$. Those particles are expected to be in a lower momentum region where the $d E / d x$ measurement is effectively useful.

\section{Gas Calorimetry}

The Texas A\&M group continues to shoulder a major share of the tasks to maintain the gas calorimetry system which is used in CDF for $\theta \leq 30^{\circ}$. The calibration of the gas calorimetry is completed.

CDF plans to maintain the gas calorimetry system in a state of readiness for use in the 1995 run in the event that the upgrade calorimetry cannot be completed in time for the run. The TAMU group (Profs. Kamon, McIntyre and Webb, and Drs. Scarpine and Wolinski) will continue to provide for the maintenance of the gas calorimetry until it is replaced.

\section{Tracking Upgrade}

We believe in the necessity of upgrading the CDF tracking system to increase the $b$ physics ability in the future collider runs: we are actively working with the CDF Tracking Upgrade Working Group. This group is charged with the task of evaluating the performance of the present tracking system for the future collider runs and its necessary modification and/or upgrade.

As a central activity, we proposed to use the gas microstrip chambers for the SVXII disk system. In our proposal ${ }^{10}$, there are several important aspects we have to study. Therefore, we would like to develop several prototype chambers for the study and request $\$ 67,000$ for the remanufactured Karl Suss MA-6 aliner ${ }^{11}$. Texas A\&M University will provide $\$ 33,000$ of cost-sharing, contingent upon the DOE funding approval. This request is pivotal to the fabrication and test of large-size chambers during 1993-1994.

The program will be carried out by Drs. Kamon, McIntyre and Trost ${ }^{12}$ and our research staff (Y. Pang, T. Elliott, T. Mazumdar). The physics simulation using GEANT3

\footnotetext{
${ }^{10}$ This proposal was written with Prof. A.Sill (Texas Tech University) who is currently a Fermilab visitor and working on SVX-II stage 1.

${ }^{11}$ We have located a used MA-6 which Karl Suss will remanufacture for us at a total cost of $\$ 100,000$.

${ }^{12}$ Dr. H.-J. Trost is presently not a CDF member.
} 
will be made by Drs. Scarpine and Wolinski and Mr. Lu.

\section{Plug Upgrade Calorimetry}

$\mathrm{CDF}$ is planning to replace the gas calorimetry system in the plug and forward regions with a new plug assembly using scintillating tile calorimetry. This replacement is necessary for taking data fast enough in future high luminosity collider runs. We will continue to work on a detailed physics simulation with the GEANT3 package in order to evaluate the performance of the calorimeters: especially identification of low $p_{T}$ electrons. This is strongly coupled with the design of the tracking device for the SVX-II stage 2 disk system. Therefore, this simulation work will be carried out in conjuction with the tracking upgrade.

\section{Summary}

All the activities described above are important for a successful CDF program: especially (a) partial $B$ meson reconstruction and particle identification by the $d E / d x$ method for physics analyses; (b) development of gas microstrip chamber for the SVX-II disk system.

Thus, it is crucial for our group to have strong support for faculty (T.Kamon, P.McIntyre, R.Webb), two postdoctoral associates (V.Scarpine, J.Wolinski) and three graduate students (J.Done, M.Kokkoris, J.Lu) in the next couple of years. 


\section{References}

[1] L. Keeble, "A Study of Four-Jet Events and Search for Double Parton Interactions with the Collider Detector at Fermilab," Ph.D. Thesis, Texas A\&M University (1992) (unpubiished); DOE-ER40633-1.

[2] T. Kamon et al., "Top Quark Search through Partial B Meson Reconstruction (I)," CDF Note 1595 (1991).

[3] J. Wahl, "Top Quark Search through Partial B Meson Reconstruction (II)," B.S. Thesis, Texas A\&M University (1992) (unpublished).

[4] M. Kokkoris, "Summary of Summer Work on the CTC $d E / d x$ Measurement," TAMU/CDF Note 92/2, 31 July 1992 (unpublished).

[5] J. Gunion and Z. Kunszt, Phys. Lett. B 159, 167 (1985); S. Parke and T. Taylor, Nucl. Phys. B 269, 410 (1986); Z. Kunszt, Nucl. Phys. B 271, 333 (1986); J. Gunion and Z. Kunszt, Phys. Lett. B 176, 163 and 477 (1986); J. Gunion and J. Kalinowski, Phys. Rev. D 34, 2119 (1986); S. Parke and T. Taylor, Phys. Rev. D 35, 313 (1987); F.A. Berends and W. Giele, Nucl. Phys. B 294, 700 (1987); M. Mangano, S. Parke and Z. Xu., Nucl. Phys. B 298, 673 (1988); M. Mangano and S. Parke, Nucl. Phys. B 299, 190 (1987).

[6] Z. Kunszt, Phys. Lett. B 145, 132 (1984); Z. Kunszt and W.J. Stirling, Phys. Lett. B 171, 307 (1986); L. Ametller, N. Paver and D. Treleani, Phys. Lett. B 169, 289 (1986).

[7] R.D.Carlitz, "Parton Correlation Functions," University of Pittsburgh preprint PITT88-05 (1989), unpublished.

[8] B. Humpert and R. Odorico, Phys. Lett. B 154, 211 (1985).

[9] AFS collaboration, T. Åkesson et al., Z. Phys. C 34, 163 (1987).

[10] UA2 collaboration, J. Alitti et al., Phys. Lett. B 268, 145 (1991).

[11] CDF Collaboration, F. Abe et al., Nucl. Instr. and Meth. A 267, 272 (1988).

[12] F. Bedeschi et al., Nucl. Instr. and Meth. A 268, 50 (1988).

[13] F. Snider et al., Nucl. Instr. and Meth. A 268, 75 (1988). 
[14] CDF Collaboration, F. Abe et al., Phys. Rev. D 45, 1448 (1992).

[15] CDF Collaboration, F. Abe et al., Phys. Rev. D 45, 3921 (1992).

[16] R. Field and R. Feynman, Nucl. Phys. B 136, 1 (1978).

[17] A modified version of the ISAJET fragmentation scheme was employed, F. Paige and S. Protopopescu, Report No. BNL-38034, 1986 (unpublished); and in "Physics of the Superconducting Super Collider," Proceedings of the Summer Study, Snownass, Colorado, 1986, edited by R. Donaldson and J. Marx (Division of Particles and Fields of the APS, New York, 1987), p. 320.

[18] CDF collaboration, F. Abe et al., Phys. Rev. Lett. 68, 1104 (1992).

[19] Z. Kunszt and W.J. Stirling, Phys. Rev. D 37, 36 (1988).

[20] C.J. Maxwell, Phys. Lett. B 192, 190 (1987).

[21] Ian Hinchliffe, private communication.

[22] Yu L. Dokshitzer, Zh. Eksp. Teor. Fiz. 73, 1216 (1977).

[23] N. Paver and D. Treleani, Nuovo Cimento A 70, 215 (1982); A 73392 (1983).

[24] L.V. Gribov, E.M. Levin and M.G. Ryskin, Phys. Rep. 100, 1 (1983).

[25] A.H. Mueller and J. Qiu, Nucl. Phys. B 268, 427 (1986).

[26] D. Brown, "A Search for Double Parton Interactions in Proton-Antiproton Collisions at $1.8 \mathrm{TeV}$," Ph.D. thesis, Harvard University (1989) (unpublished).

[27] CDF collaboration, F. Abe et al., Phys. Rev. D 44, 29 (1991).

[28] J. Morfin and W.K. Tung, Zeit. Phys. C 52, 13 (1991).

[29] P. Langacker, Phys. Rev. Lett. 63, 1920 (1989).

[30] Particle Data Group, "Review of Particle Properties," Phys. Rev. D 45 (1992).

[31] C. Bebek et al., "Exclusive Decays and Masses of the $B$ Mesons," Phys. Rev. D 36, 1289 (1987).

[32] C. Albajar et al., Phys. Lett. B 273, 540 (1991). 
[33] CDF Collaboration, F. Abe et al., submitted to Phys. Rev. Lett. (1992).

[34] J. Wolinski et al., "Testbeam 1991: FHA Calibration," CDF Note 1695 (1992).

[35] T. Baumann et al., "Testbeam 1990: Gas Calorimeter Absolute CARROT Calibration," CDF Note 1697 (1992).

[36] J. Cunningham, L. Keeble, "Temperature Transducer Performance and Calibration after the 1990 Test Beam Run," CDF Note 1784 (1992);

J. Cunningham, K. Sumorok, "Temperature Transducer Performance and Calibration Part II." CDF Note 1785 (1992);

J. Cunningham, "Temperature Transducer Calibration Box Resistance Stability," CDF Note 1786 (1992).

[37] J. Cunningham, K. Sumorok, "Temperature \& Pressure Monitoring for the Gas Calorimetry l"eeuback HV Systemi," CDF Note 1787 (1992).

[38] T. Kamon, "FILT_GAS Tuning 92," (in preparation). Original work is summarized in D. Brown, M. Franklin, "FILT_GAS, A Calorimetry Cleanup Module," CDF Note 696 (1988): D. Brown, M. Franklin, "FILT_GAS 88," CDF Note 811 (1988).

[39] T. Kamon, "EWE Threshold Effect on FHA Energy Scale," CDF Note 859 (1989).

[40] S. Behrends, private communication.

[41] T. Kamon, T. Bowcock, and R. Webb, "Forward Hadron (Gas) Calorimeter: The Future," CDF Note 972 (1989).

[42] S. Cihangir et al., Nucl. Instr. and Meth. A 217, 249 (1988).

[43] J. Wahl, "GEANT3 Guide for the CDF Calorimetry," CDF Note 1710 (1992).

[44] T. Kamon et al., "FHA Status in 1992 Run," Presentation at the CDF Collaboration Meeting, Fermilab, Batavia, IL., 23 September 1992.

[45] T. Kamon et al., "Exploration of the Use of Gas Microstrip Chamber Technology in the SVX-II Disk System," CDF Note 1834 (1992).

[46] CDF Collaboration, "SVX II Conceptual Design Progress Report," CDF Note 1764 (1992). 
[47] A. Oed, Nucl. Instr. and Meth. A 263, 351 (1988).

[48] F. Angelini et al., Nucl. Instr. and Meth. A 283, 755 (1989).

[49] F. Angelini et al., Preprint INFN PI/AE 92/01 (INFN Pisa, Italy), 14 April 1992; Proceedings of the 6th International Wire chamber Conference, Vienna, 17-21 February 1992.

[50] R. Bouclier et al., Proceedings of the 6th International Wire chamber Conference, Vienna, 17-21 February 1992.

[51] E.F. Barasch et al., SDC-92-318 (Texas A\&M University, College Station, TX), 8 September 1992; Proceedings of the 3rd International Conference for Advanced Technology and Particle Physics, Como, Italy, 22-26 June 1992.

[52] E.F. Barasch et al., Talk presented by H.-J.Trost at the Workshop on Charged Particle Tracking for Advanced HEP Experiments, Boulder, Colorado, 13-18 July 1992.

[53] R. Bellazzini, INFN and University of Pisa, private communication.

[54] Karl Suss America Inc., P.O.Box 157, Route 100, Suss Drive, Waterbury Center, VT 05677.

[55] SDC ITD Group, B. Foster et al., Internal Note SDC-92-171, 20 January 1992; Solenoid Detector Collaboration, E.F. Berger et al., Technical Design of a Detector to be Operated at the Superconducting Super Collider, SDC-92-201, 1 April 1992.

[56] F.G. Hartjes, Talk presented at the Workshop on Charged Particle Tracking for Advanced HEP Experiments, Boulder, Colorado, 13-18 July 1992.

[57] T. Zimmermann, Technical Note TM-1606 (Fermi National Accelerator Laboratory, Batavia, Illinois, USA), 11 July 1989, unpublished.

[58] J. Done, T. Kamon, G. Kyriazis, J. Lu and V. Scarpine, "GEANT3 Guide for the CDF Tracking System," TAMU/CDF Note 92/1, 23 July 1992 (unpublished). 

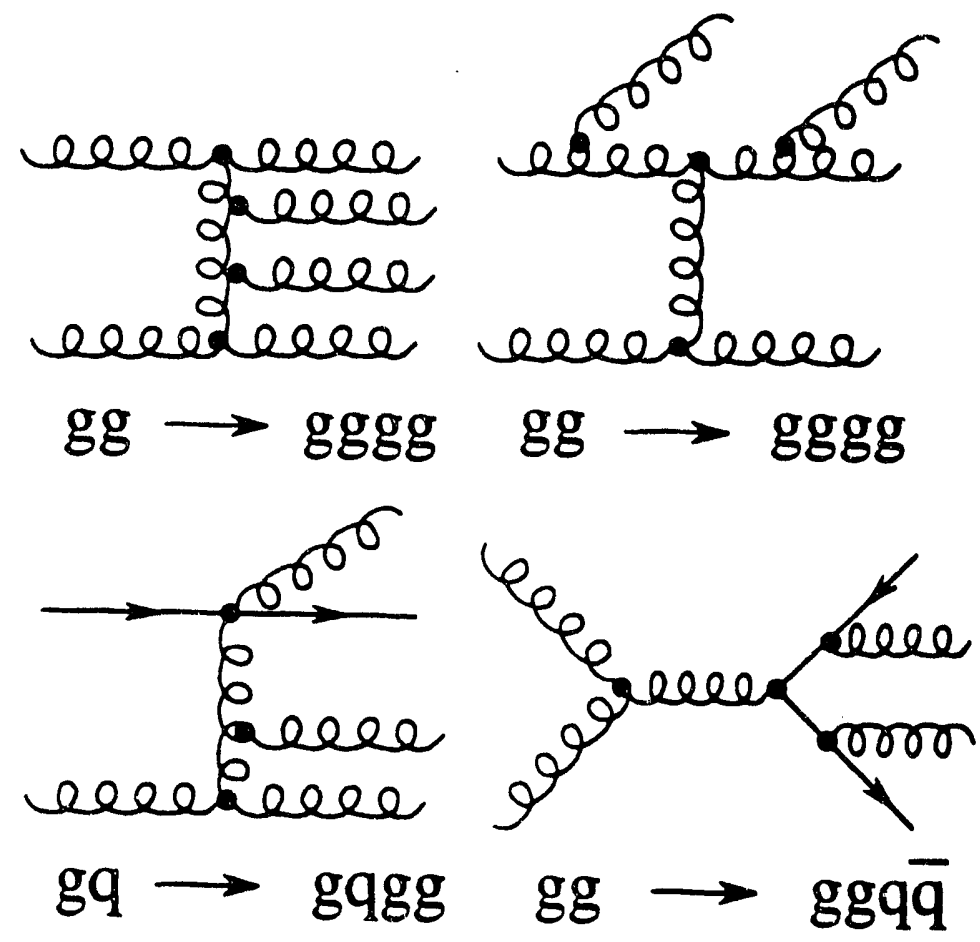

Figure 1: A few of the Feynman diagrams relevant to the the four-jet double bremsstrahlung process. 

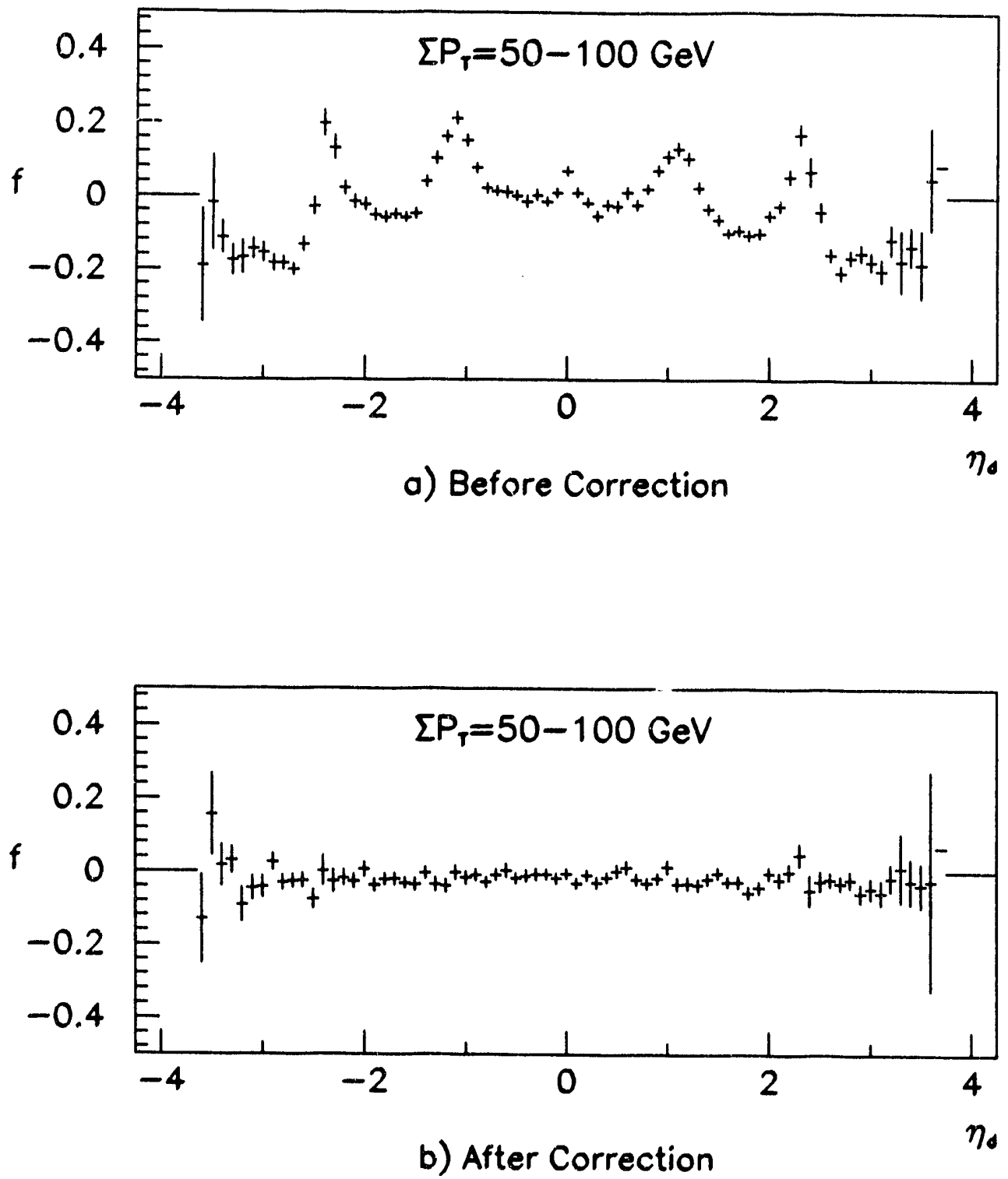

Figure 2: $f$ (see text for definition) as a function of $\eta_{d}$ for dijet data in the range $50<\sum p_{T}<$ $100 \mathrm{GeV} / \mathrm{c} \mathrm{a}$ ) before and b) after application of the relative jet correction. 


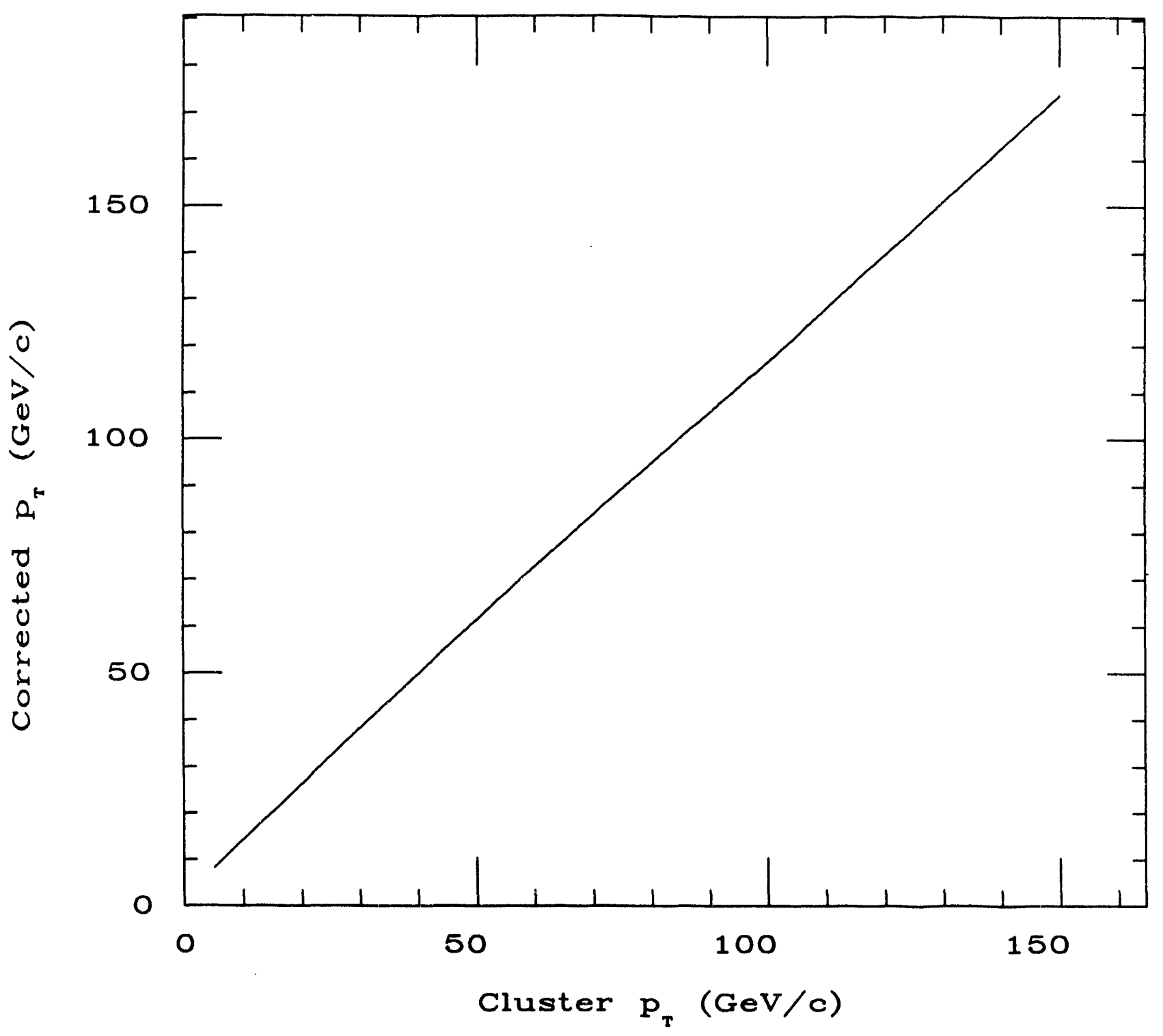

Figure 3: The absolute jet correction function in the central region, $0.2<\eta_{d}<0.7$. Shown is corrected $p_{T}$ versus clustered jet $p_{T}$, in a range of clustered jet $p_{T}$ relevant to the four-jet analysis. 


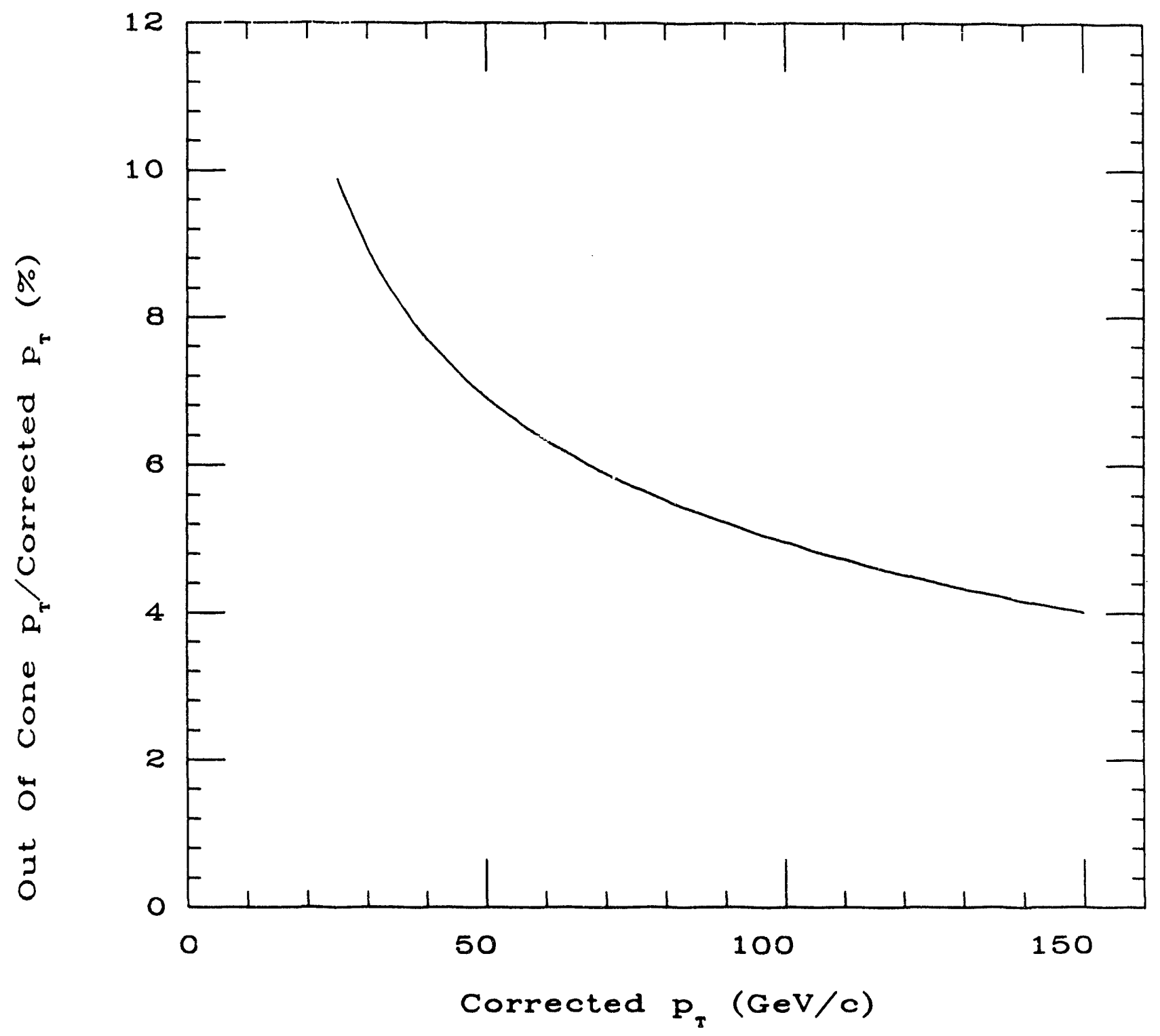

Figure 4: The ratio of out-of-cone $p_{T}$ to jet $p_{T}$ versus jet $p_{T}$ for a cluster cone of size 0.7 in simulated dijet events, using a tuned Feynman-Field fragmentation model and full detector simulation. Clusters have been confined to the region $0.2<\left|\eta_{d}\right|<0.7$, and the particles are identified as having originated from a parton that matches the cluster in $\eta-\phi$ space. 


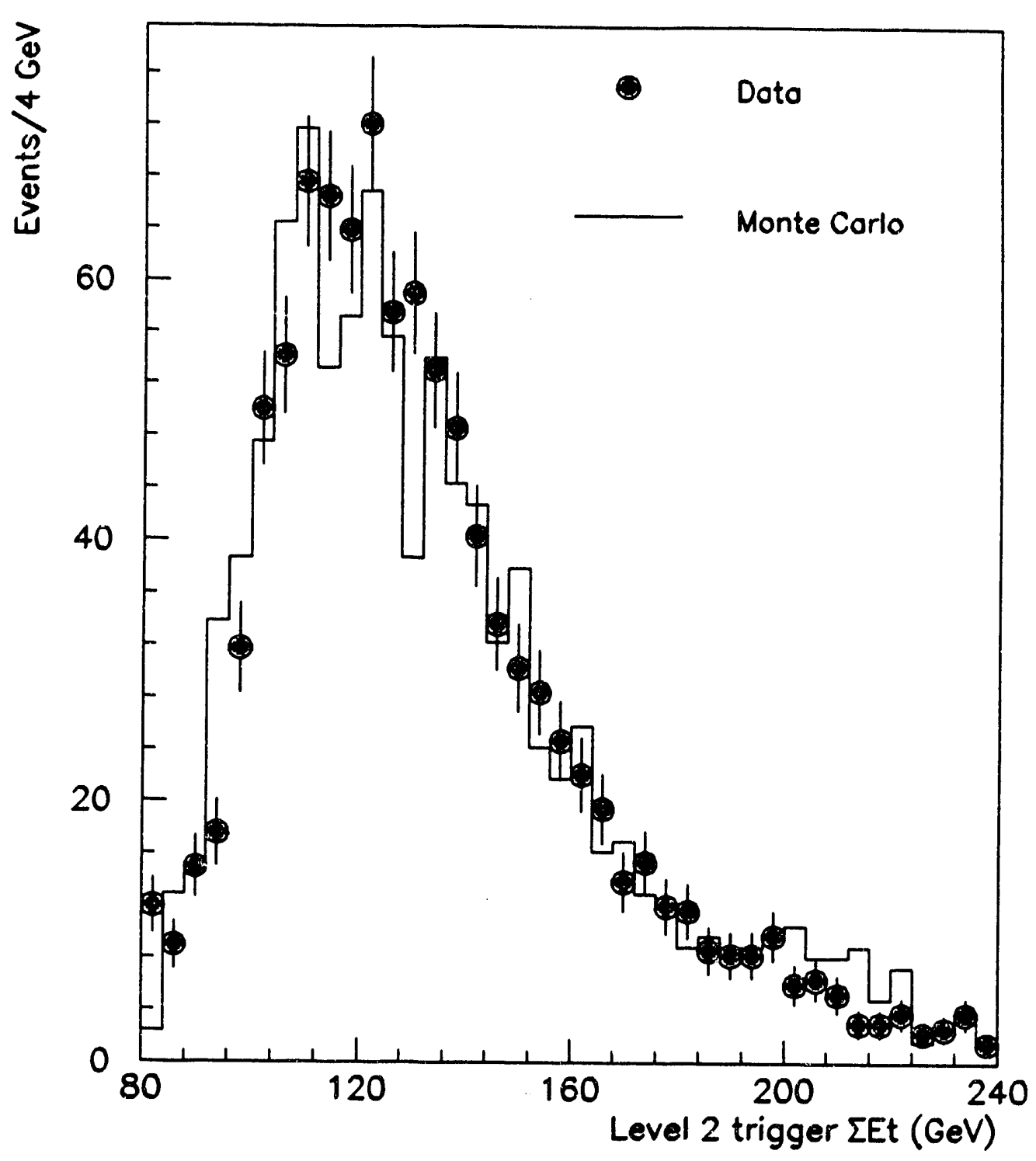

Figure : The level 2 trigger $\Sigma E_{T}$ obtained with simulated four-jet events and full detector simulatio! slaid on the distribution obtained with actual four-jet events taken with the multijet trigge. . 

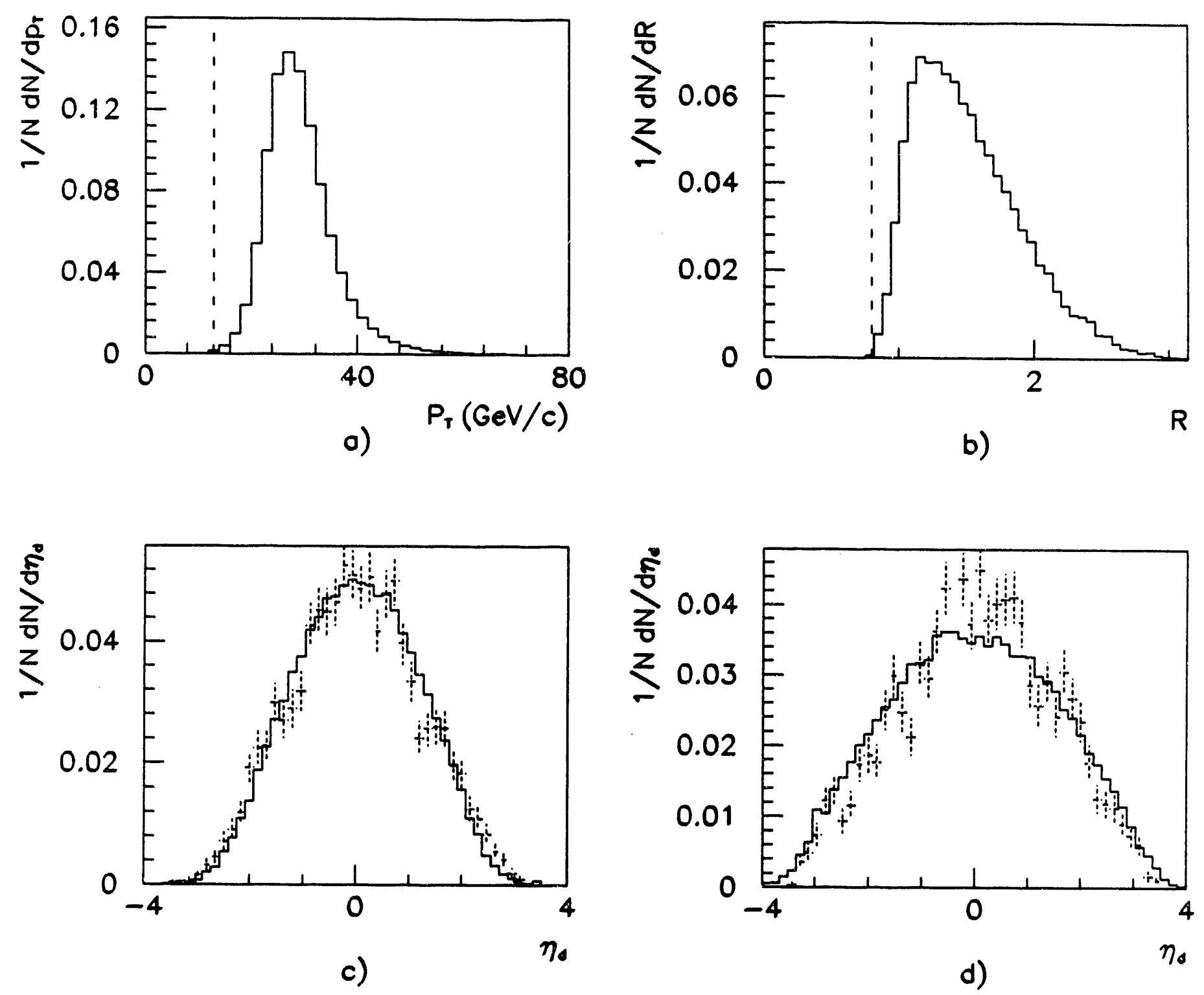

Figure 6: Distributions of a) softest parton $p_{T}$, b) minimum parton separation, c) and d) $\eta_{d}$ of the softest and highest $p_{T}$ partons respectively for the double bremsstrahlunig simulation. The fast jet simulation has been used, and the standard offline cuts have been applied to the corrected jets. For c) and d) the parton level distributions (solid) have been overlaid with the $\eta_{d}$ distributions for the softest and highest $p_{T}$ jets in the data (dashed points). 

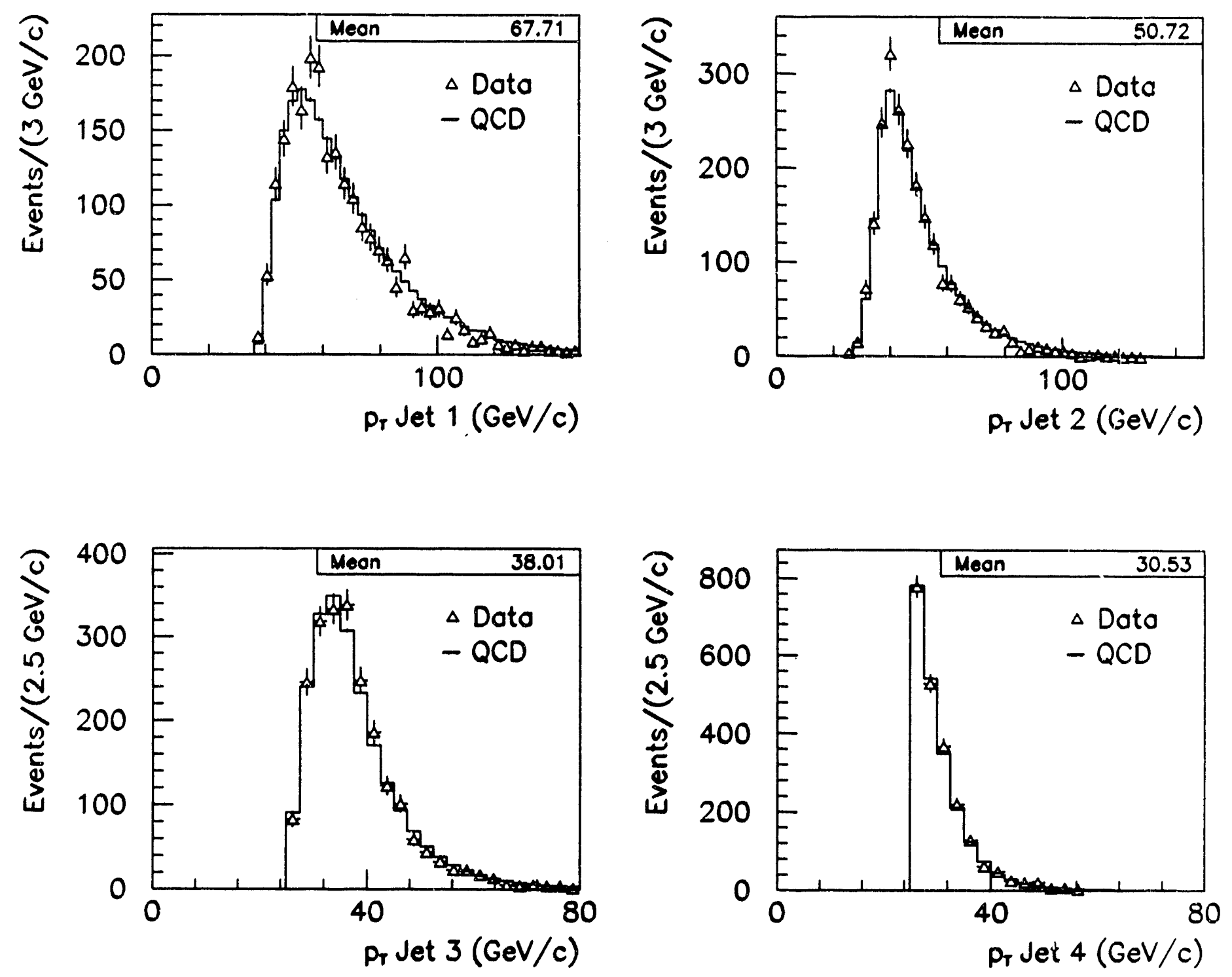

Figure 7: The $p_{T}$ spectrum for all four jets obtained with data overlaid on the predictions of leading order QCD. 


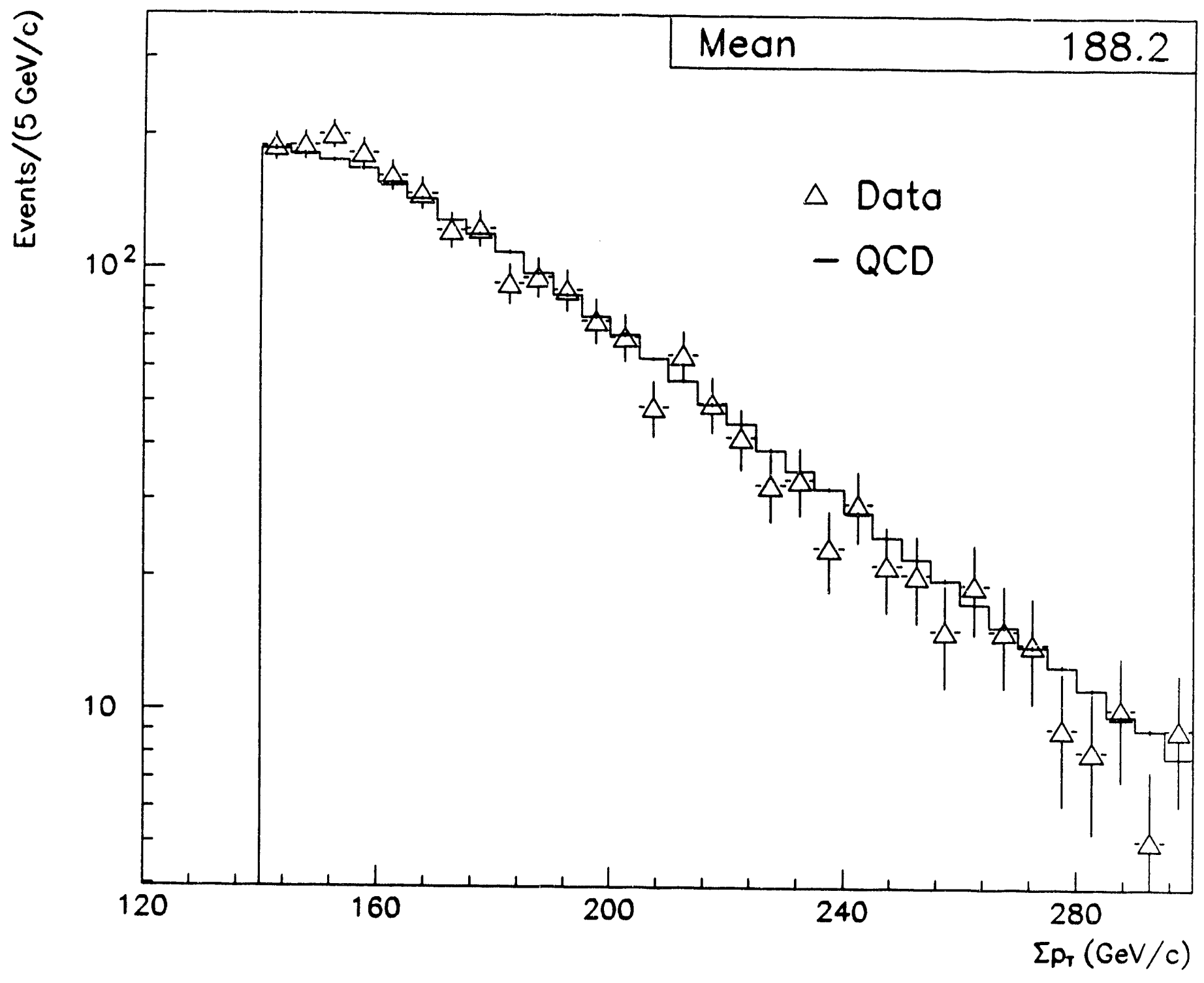

Figure 8: The scalar $\Sigma p_{T}$ spectrum for four-jet events obtained with data overlaid on the leading order QCD prediction using structure function EHLQ1 with $Q=\left\langle p_{T}\right\rangle$. 

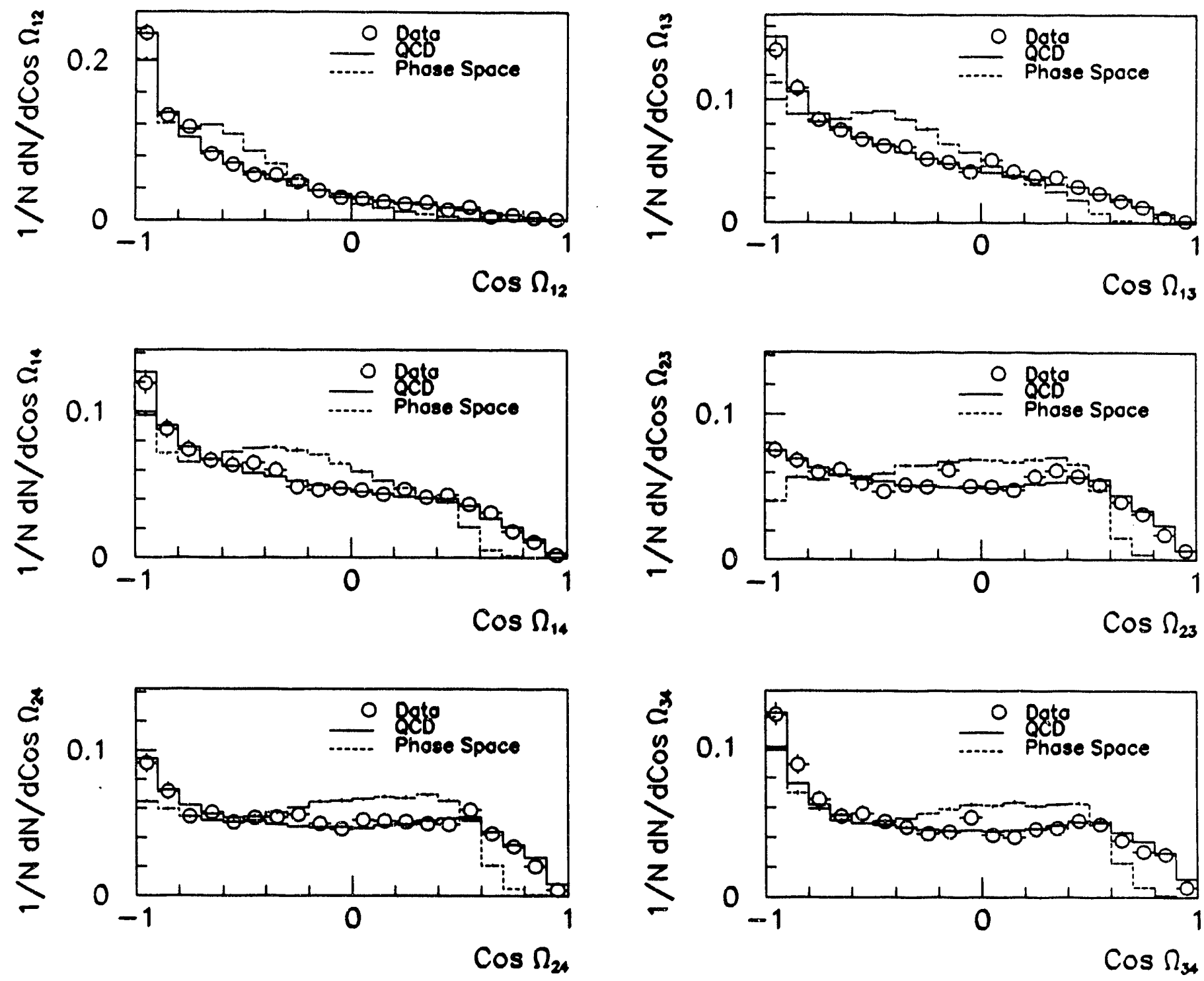

Figure 9: A comparison between data and QCD for the six angular variables $\cos \Omega_{i j}$ (defined in the text). Data points are overlaid on the leading order QCD predictions. 

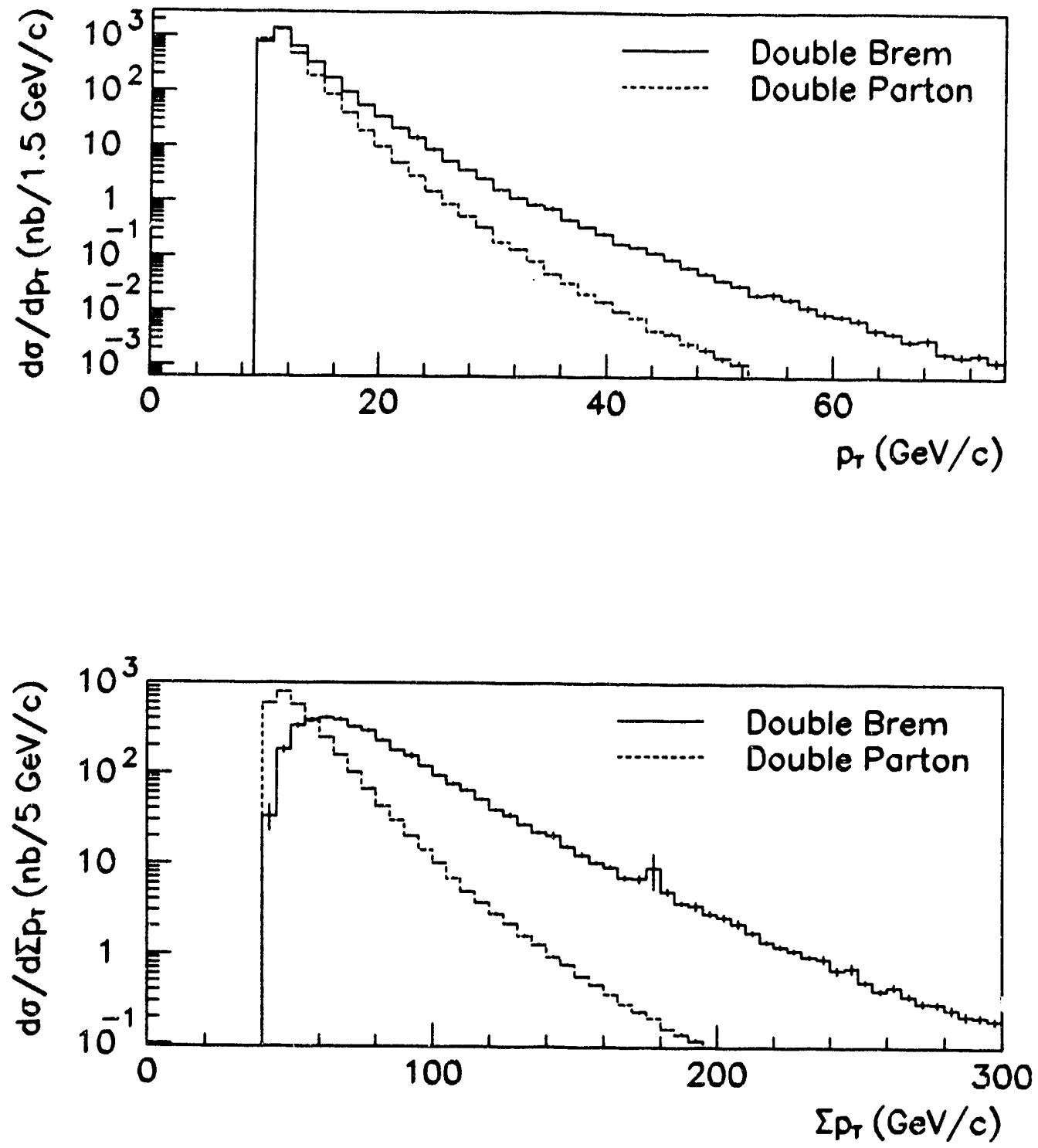

Figure 10: a) The double parton and double bremsstrahlung scattering cross sections plotted a) as a function of the $p_{T}$ of the softest parton generated, b) as a function of the scalar $\Sigma p_{T}$ of all four jets. A value of $\sigma_{\text {eff }}=8.7 \mathrm{mb}$ has been used in the double parton calculation, with structure function set EHLQ1, $Q=\left\langle p_{T}\right\rangle$. 

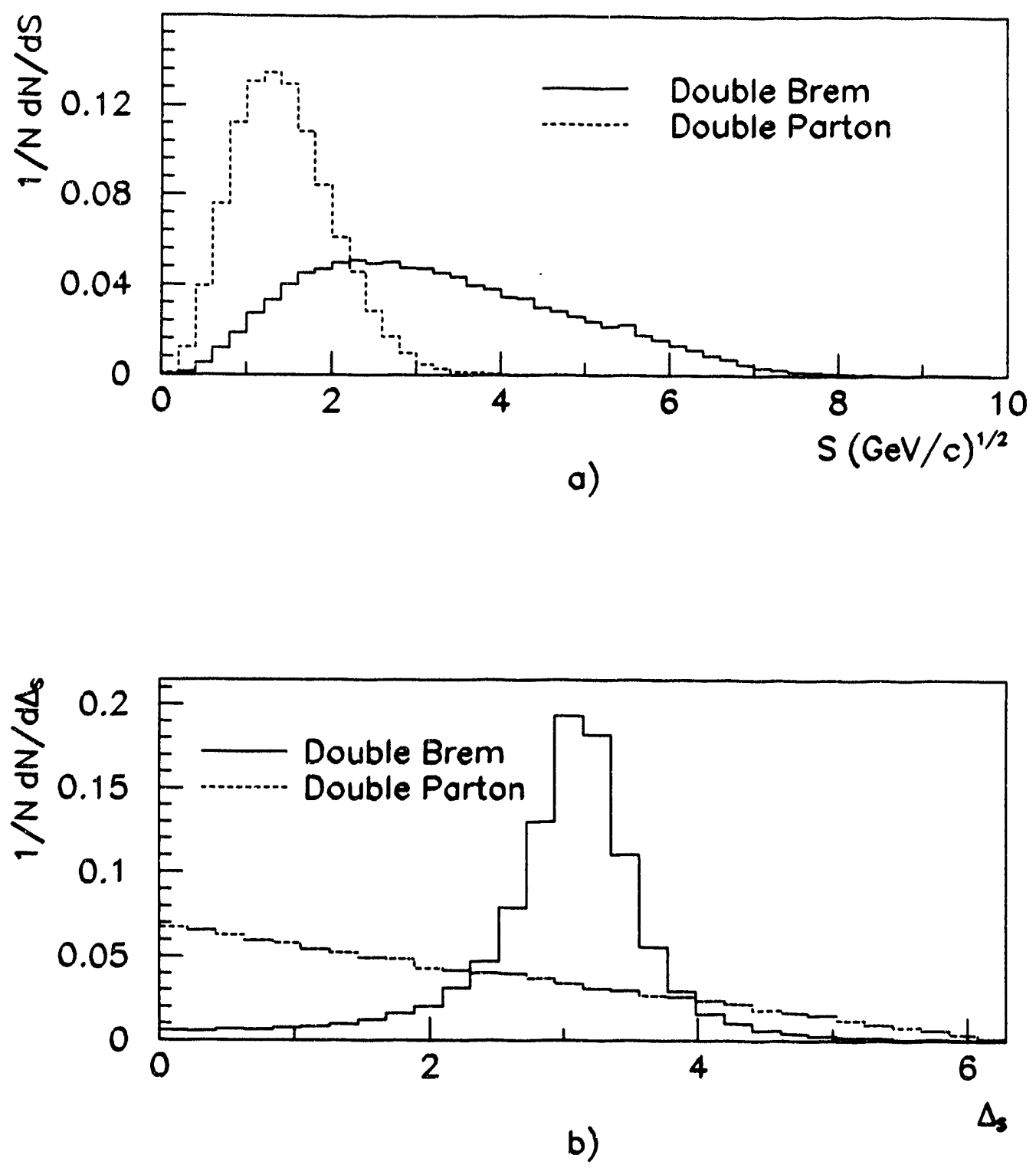

Figure 11: The distributions of a) $S$ and b) $\Delta_{S}$ for double parton and double bremsstrahlung simulated events. Detector effects are modeled using the fast jet simulation. 


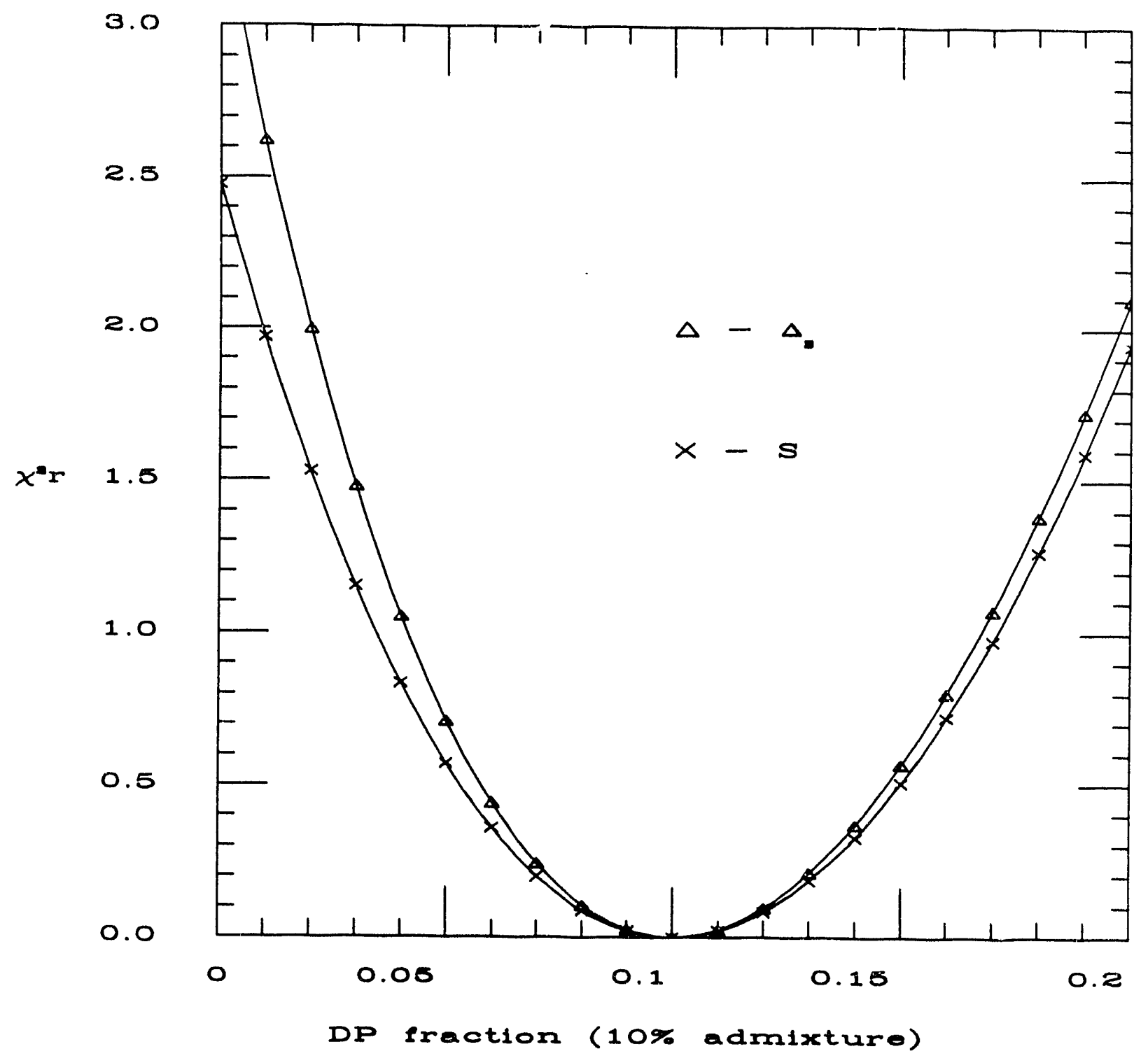

Figure 12: The $\chi_{r}^{2}$ dependence of $S$ and $\Delta_{S}$ as a function of the fraction of double parton events for an artificially created sample of $90 \%$ double bremsstrahlung and $10 \%$ double parton events. 

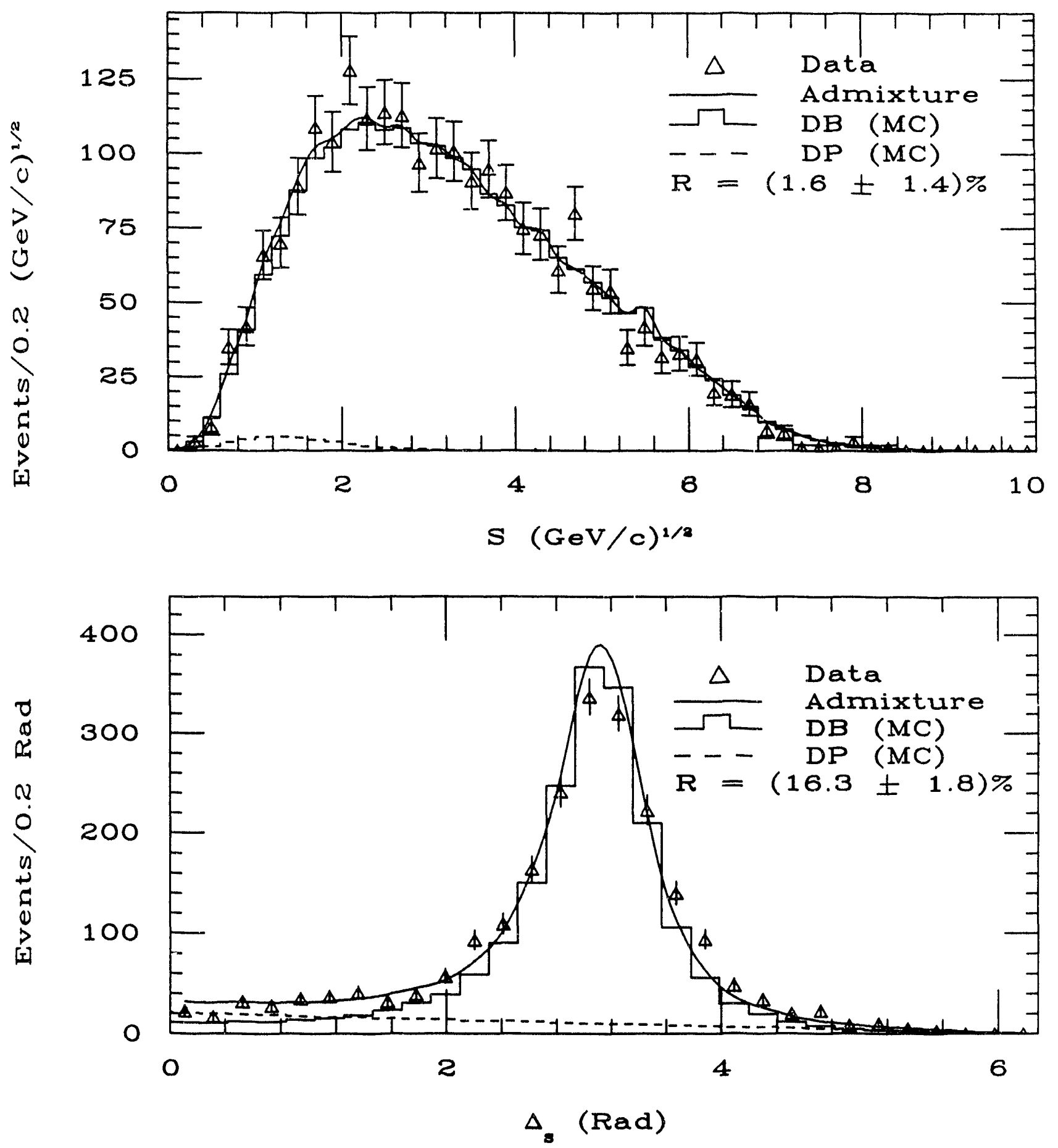

Figure 13: Distribution of a) $S$ and b) $\Delta_{S}$ for CDF four-jet events. A combined fit to an admixture of double parton and double bremsstrahlung simulated events is also shown, together with the ratio of fitted double parton to double bremsstrahlung events, $\mathcal{R}$. 

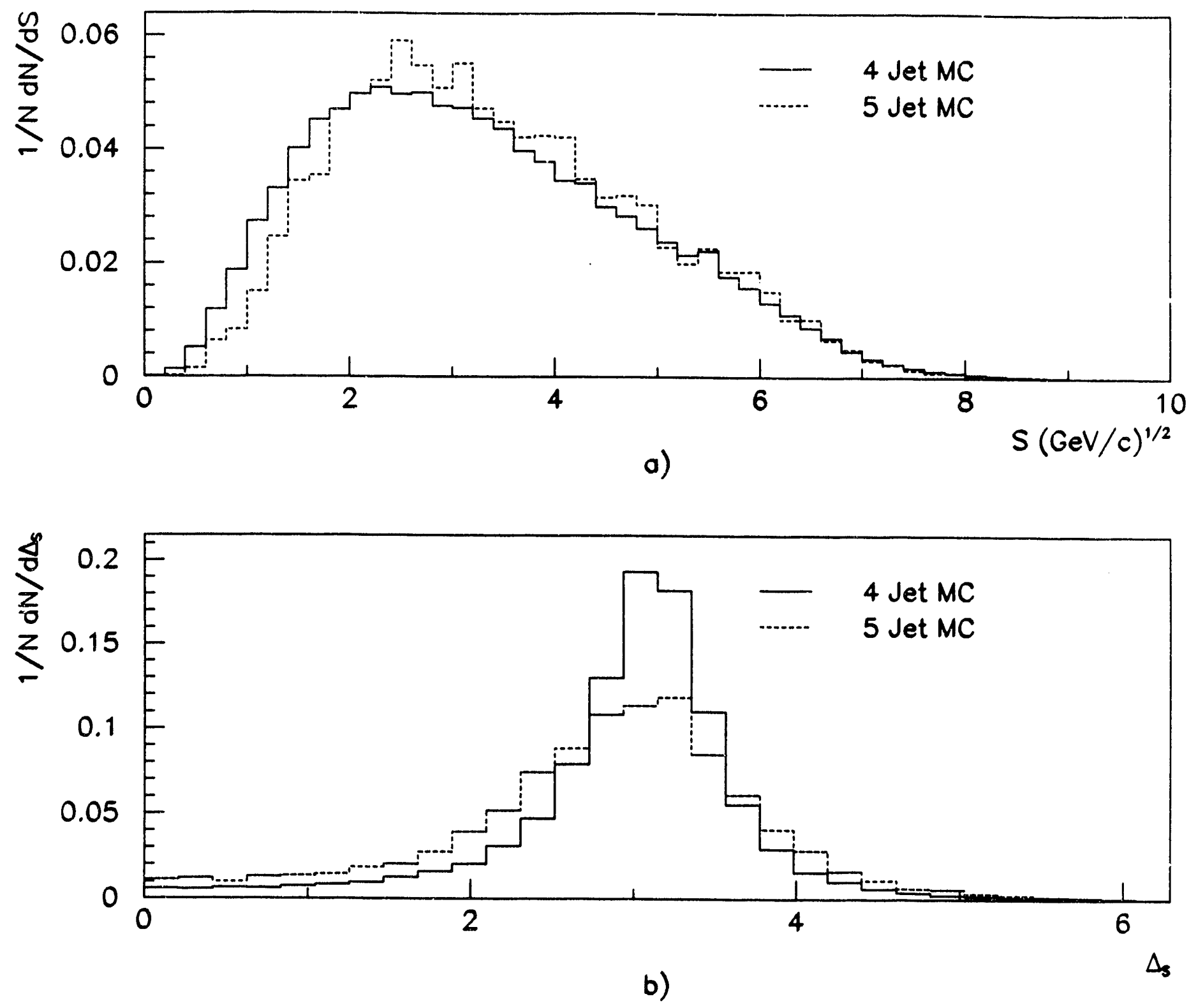

Figure 14: Distributions of a) $S$ and b) $\Delta_{S}$ obtained using simulated five-jet events, where only four jets pass the standard analysis cuts. For comparison, the distributions obtained with the four-jet simulation are also shown. 

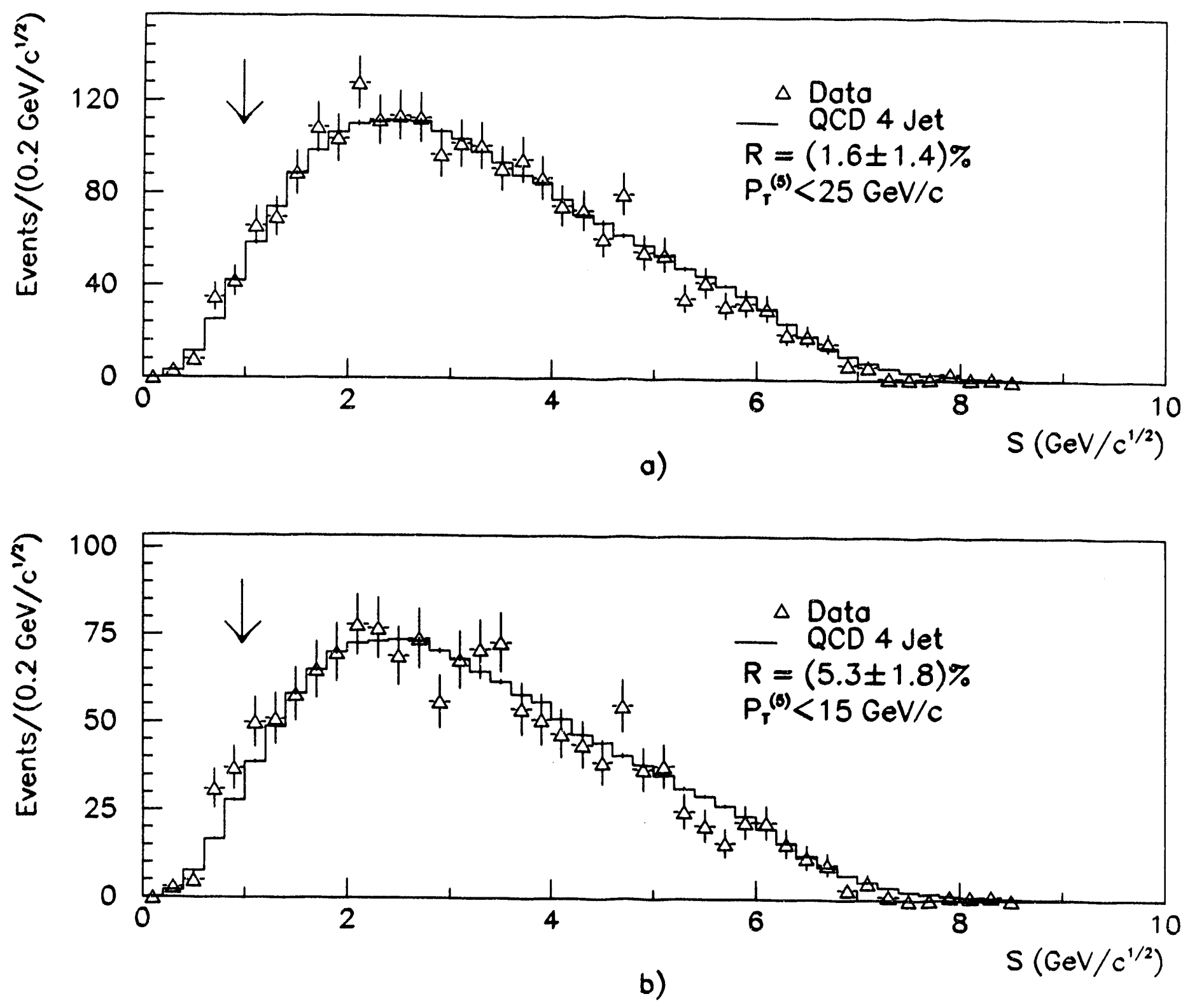

Figure 15: The $S$ variable obtained with four-jet data overlaid on the predictions of a leading order QCD double bremsstrahlung simulation with a) the default analysis cuts and b) a more severe cut on fifth clusters, $p_{T}^{(5)}<15 \mathrm{GeV} / \mathrm{c}$. 

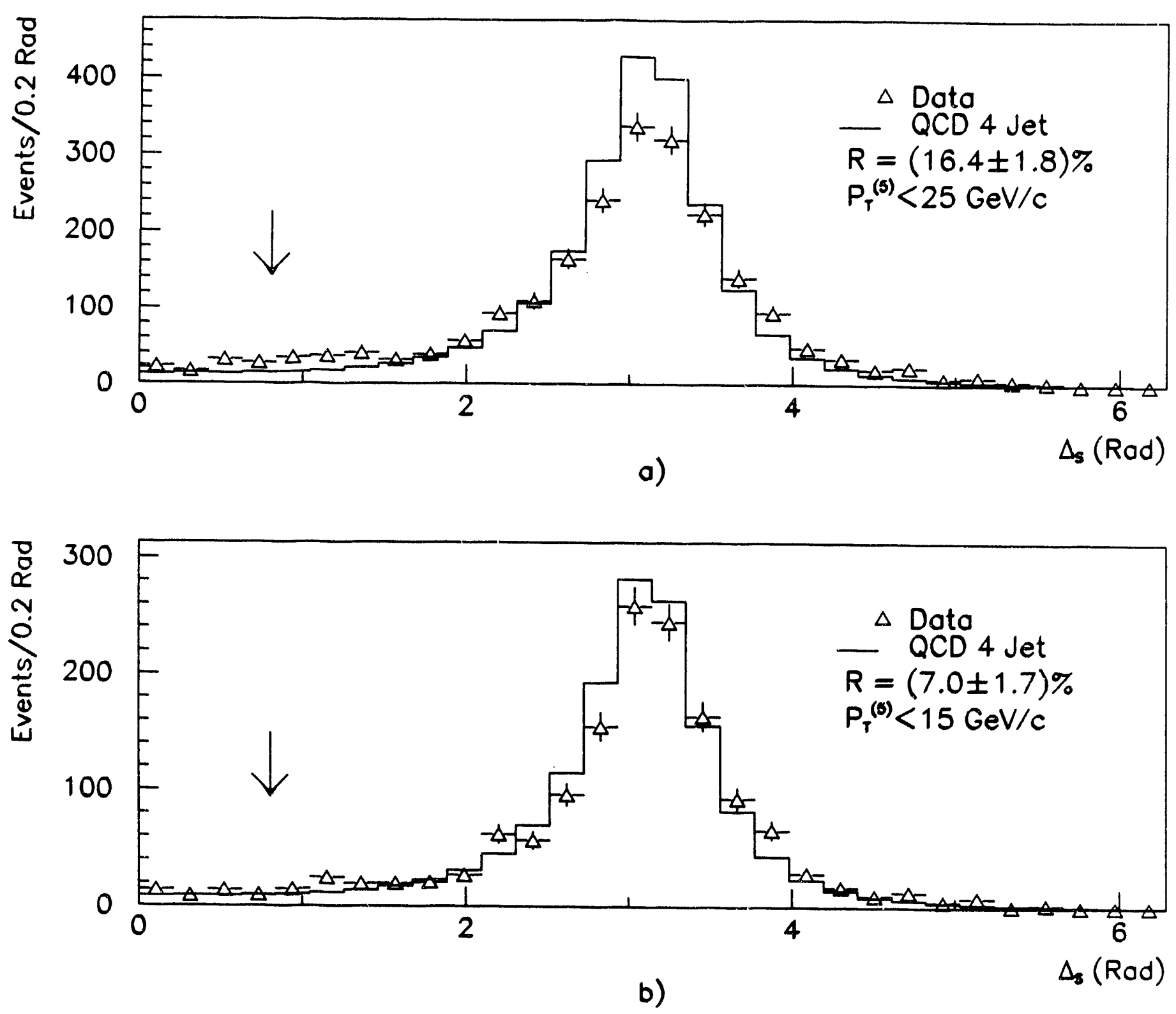

Figure 16: The $\Delta_{S}$ variable obtained with four-jet data overlaid on the predictions of a leading order QCD double bremsstrahlung simulation with a) the default analysis cuts and b) a more severe cut on fifth clusters, $p_{T}^{(5)}<15 \mathrm{GeV} / \mathrm{c}$. 


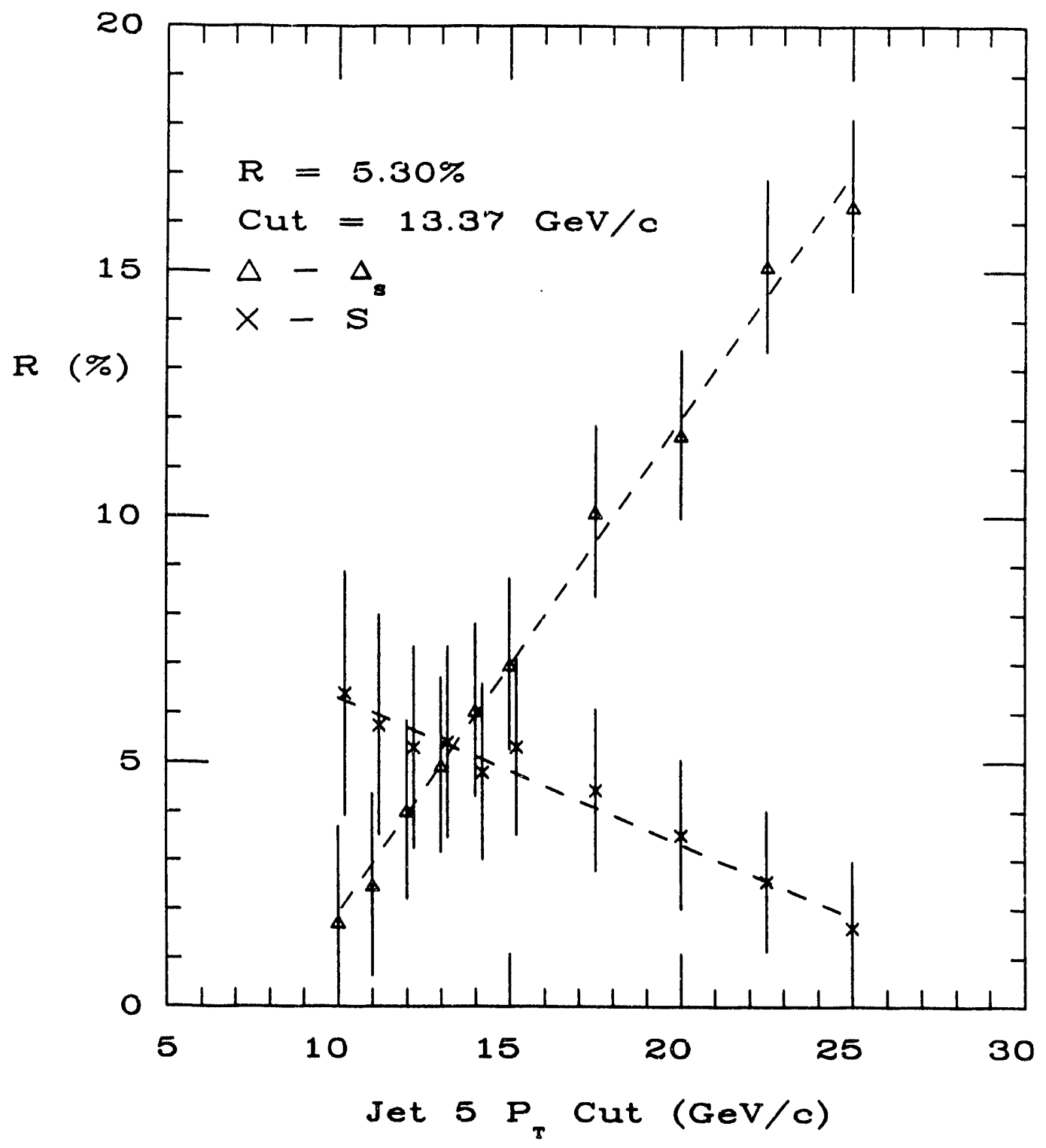

Figure 17: The double parton fraction in CDF four-jet data $\mathcal{R}$ (see text for definition) for the $S$ and $\Delta_{S}$ variables as a function of cut on the $p_{T}$ of the fifth jet. 


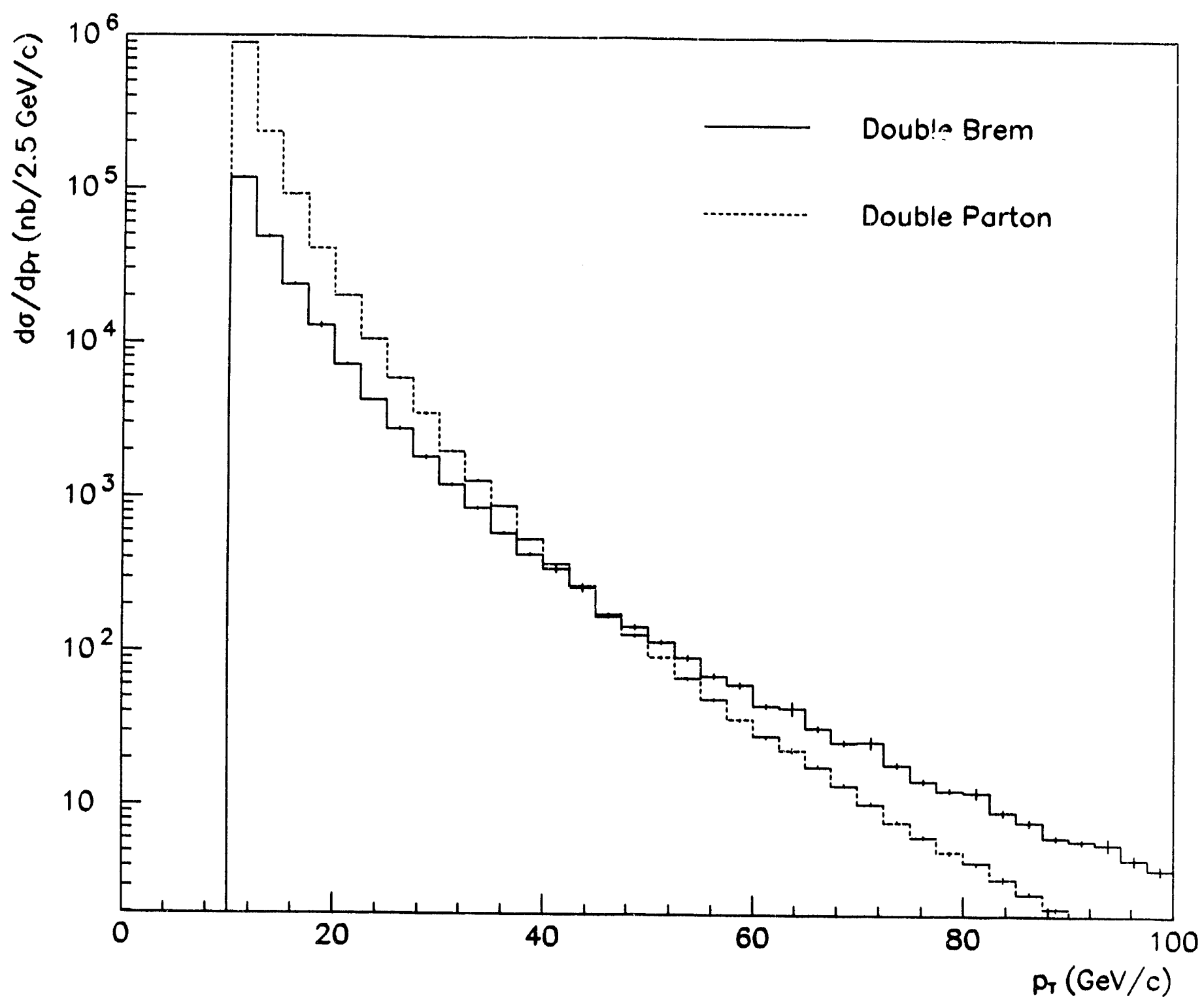

Figure 18: The double parton and double bremsstrahlung cross sections at $E_{C M}=40 \mathrm{TeV}$ as a function of the $p_{T}$ of the softest parton. 


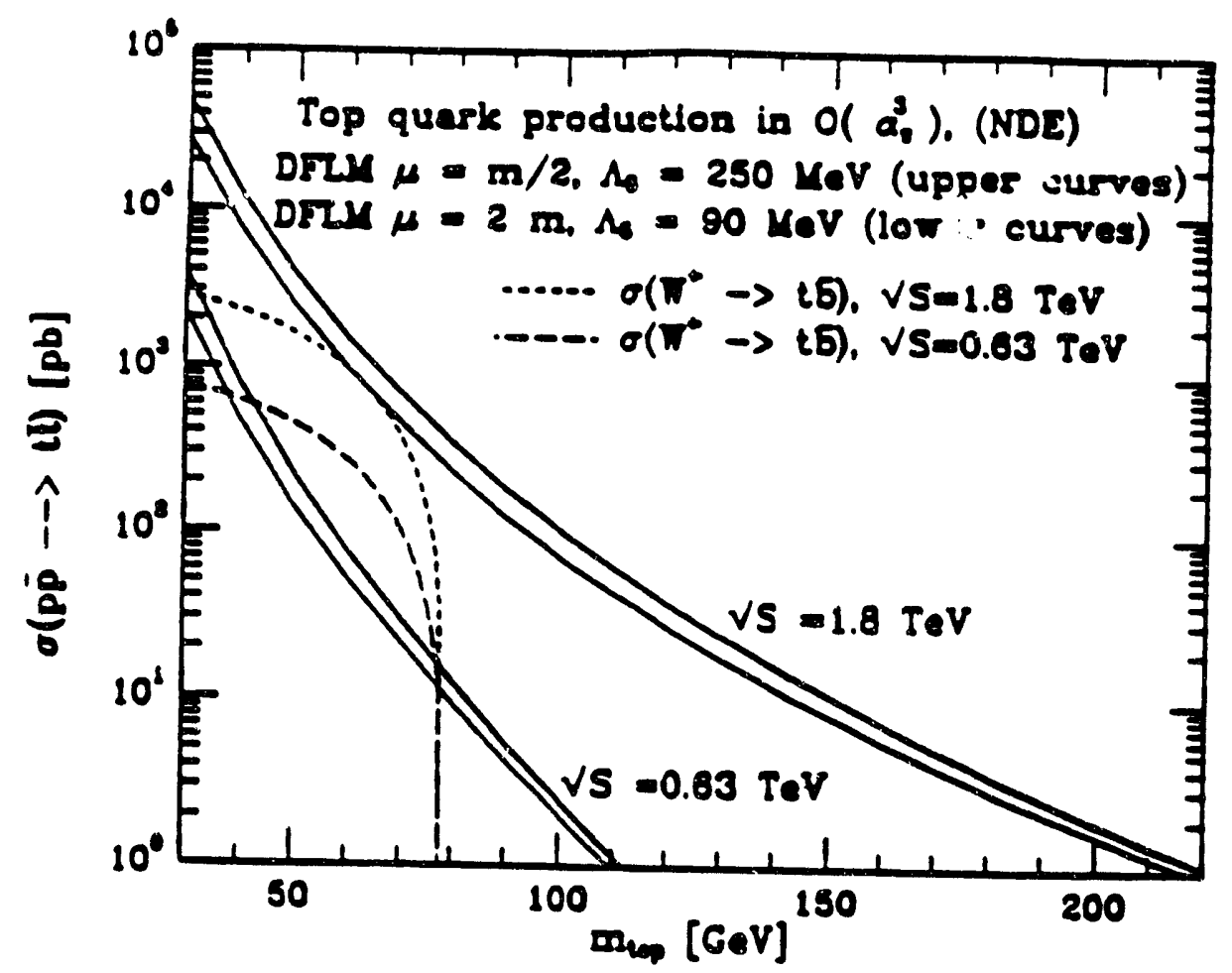

Figure 19: Cross section for top quark pair production at the Tevatron energy.

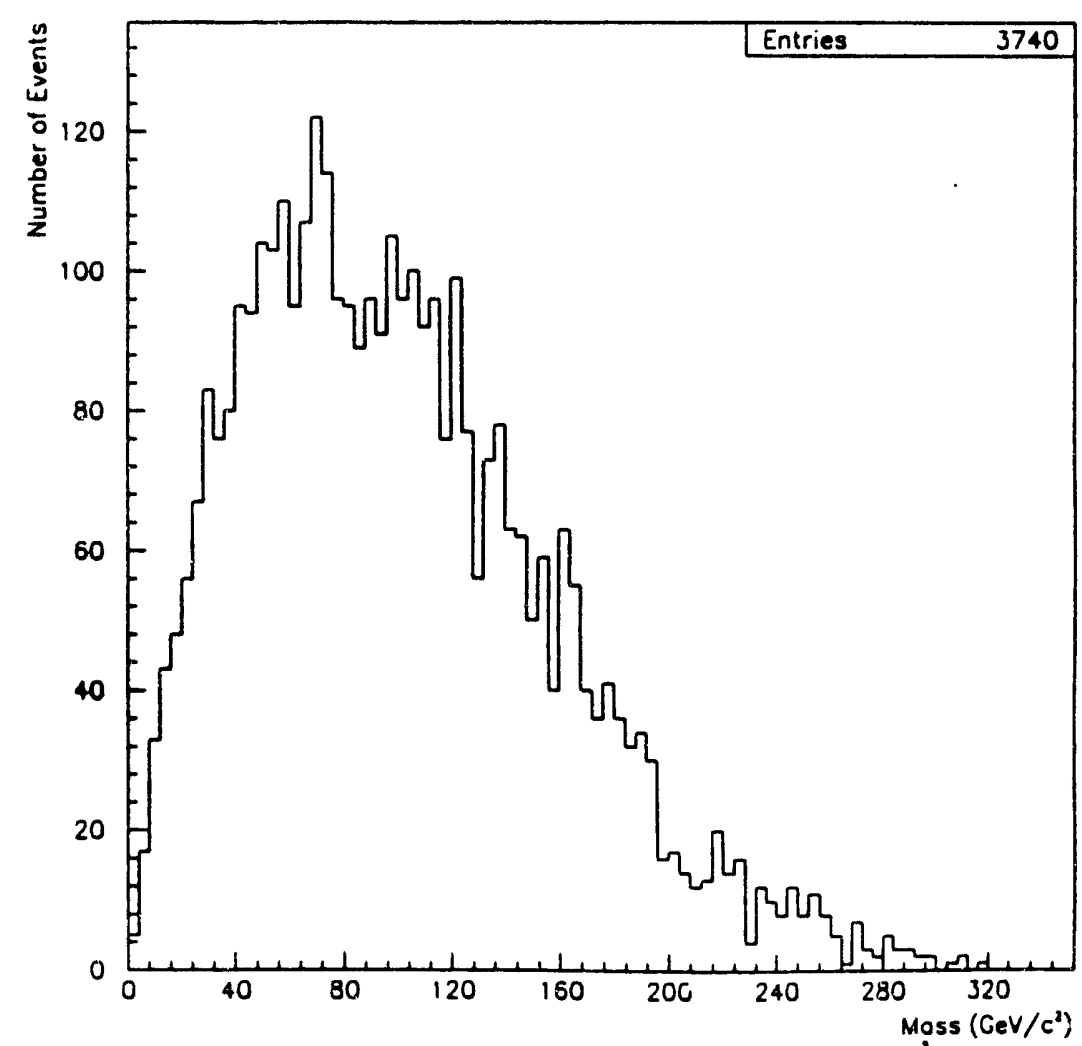

Figura 20: Dilepton mass distribution for $t \bar{t} \rightarrow \ell \ell^{\prime}+X$ with $M_{\text {top }}=120 \mathrm{GeV} / \mathrm{c}^{2}$. 

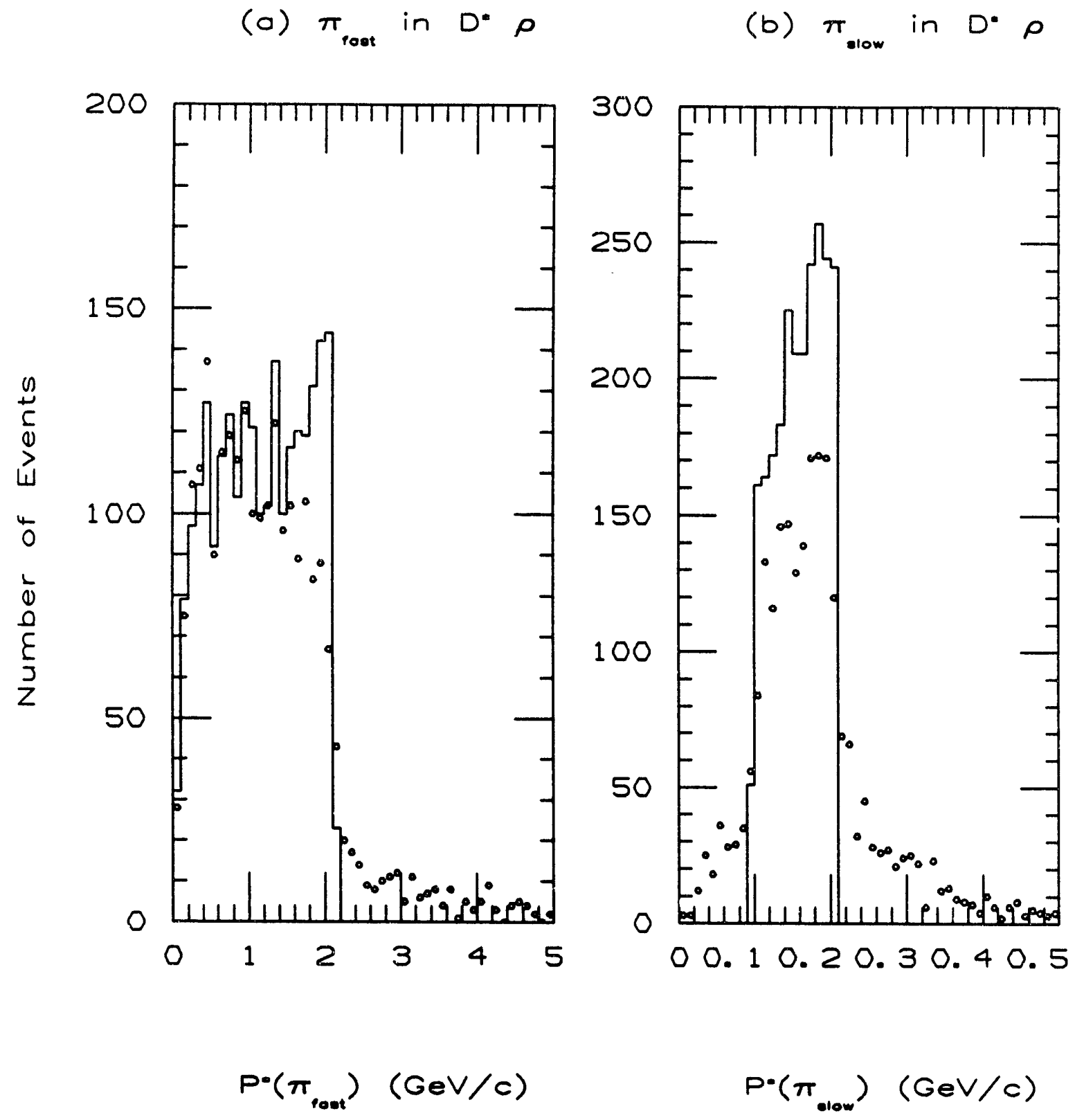

Figure 21: (a) Momentum distributions of $\pi_{f a s t}$ in the $B$-meson rest frame (solid) and jet rest frame (open circle). (b) Momentum distributions of $\pi_{\text {slow }}$ in the $B$-meson rest frame (solid) and jet rest frame (open circle). 
(a) Ideal Case

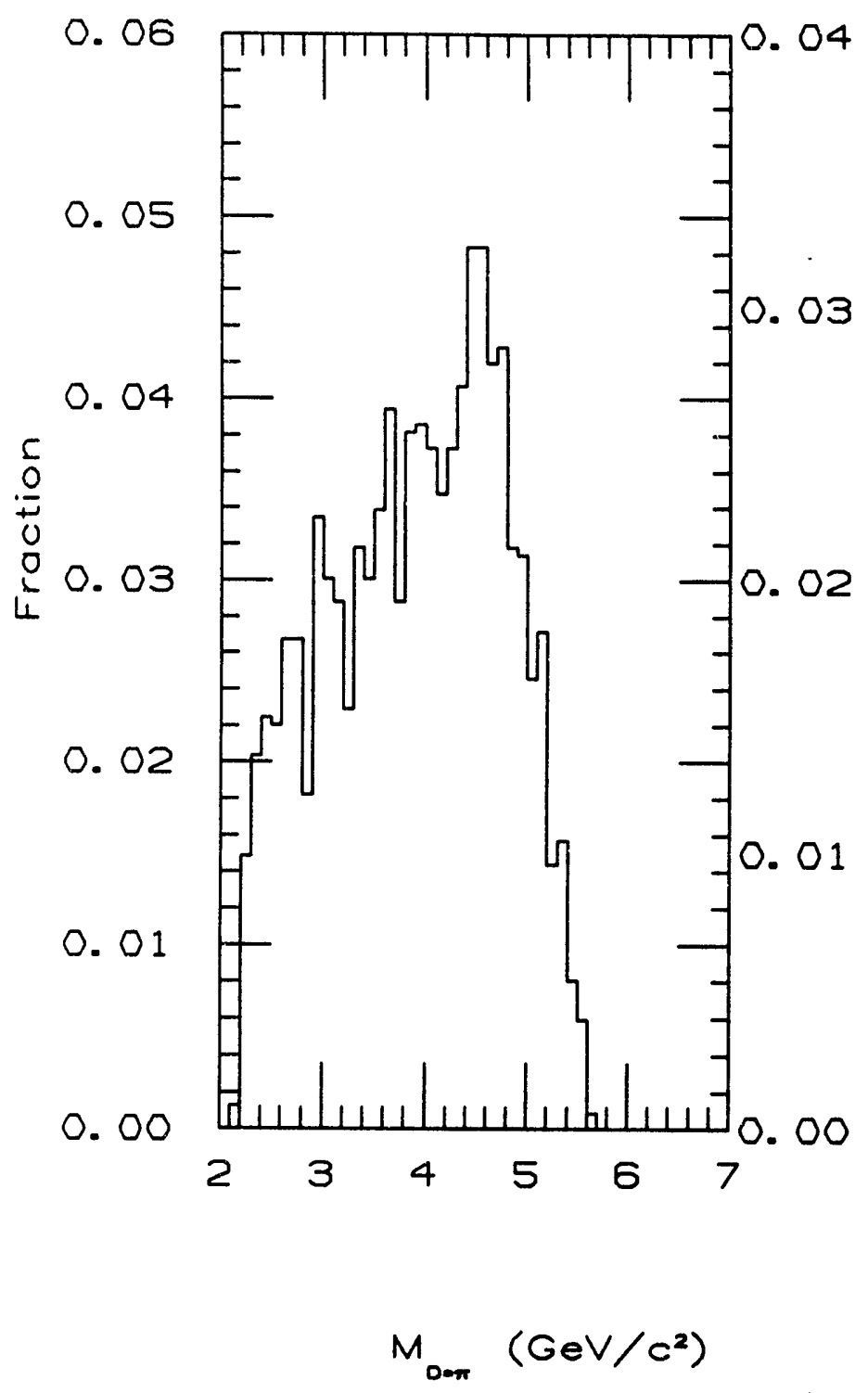

(b) Best Reconstruction

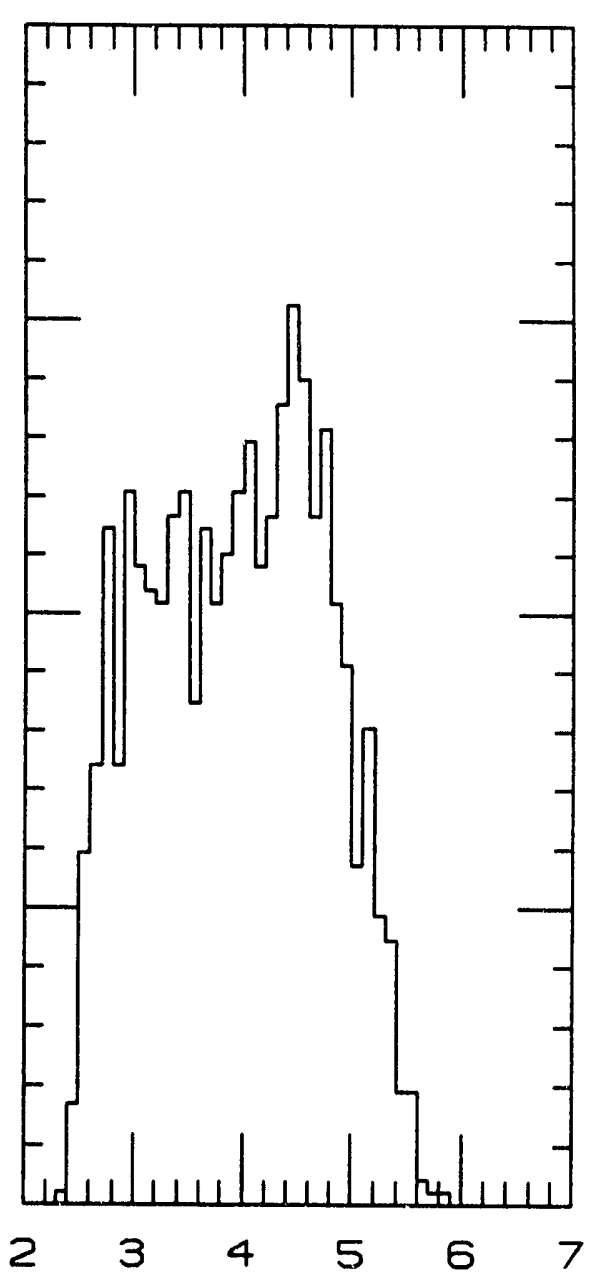

$$
M_{D \cdot \pi}\left(\mathrm{GeV} / \mathrm{c}^{2}\right)
$$

Figure 22: (a) Mass distribution of $D^{*-} \pi^{+}$system using $D^{*}$ trick for perfect particle identification. (b) Mass distribution of $D^{*-} \pi^{+}$system using $D^{*}$ trick for our technique. About $61 \%$ of a total events in (a) is reconstructed. 


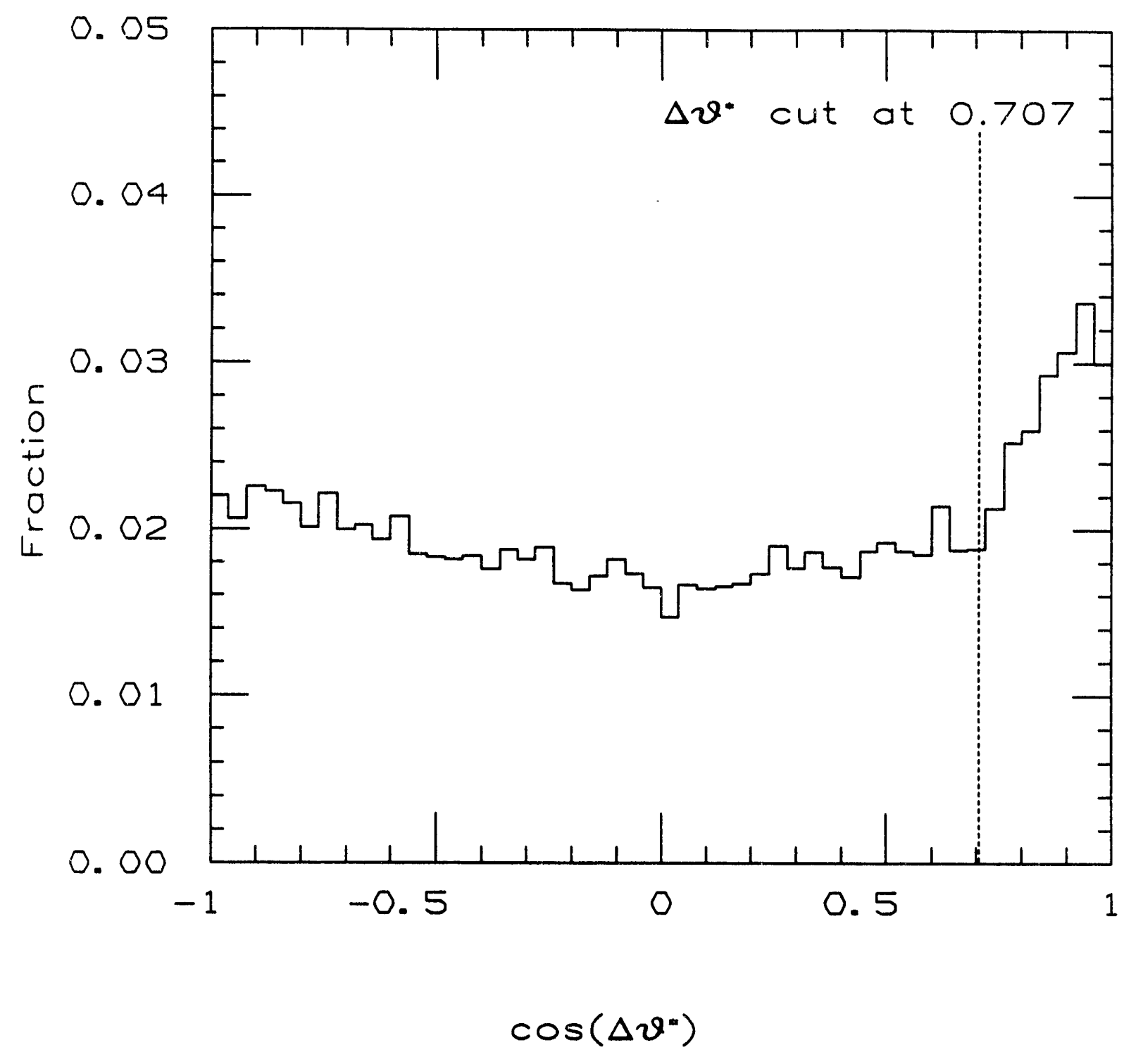

Figure 23: Distribution of $\cos \Delta \theta^{*}$ which is an opening angle between $\vec{P}^{*}\left(\pi^{0}\right)_{\text {expected }}$ and $\vec{P}^{*}(E M)$. See text for detail. An enhanced peak around $\cos \Delta \theta^{*}=1$ is for candidates of $\pi^{0}$. 
(a) $D^{*}+\pi_{\text {root }}+E M$ cluster

(b) $\pi_{\text {root }}+E M$ cluster

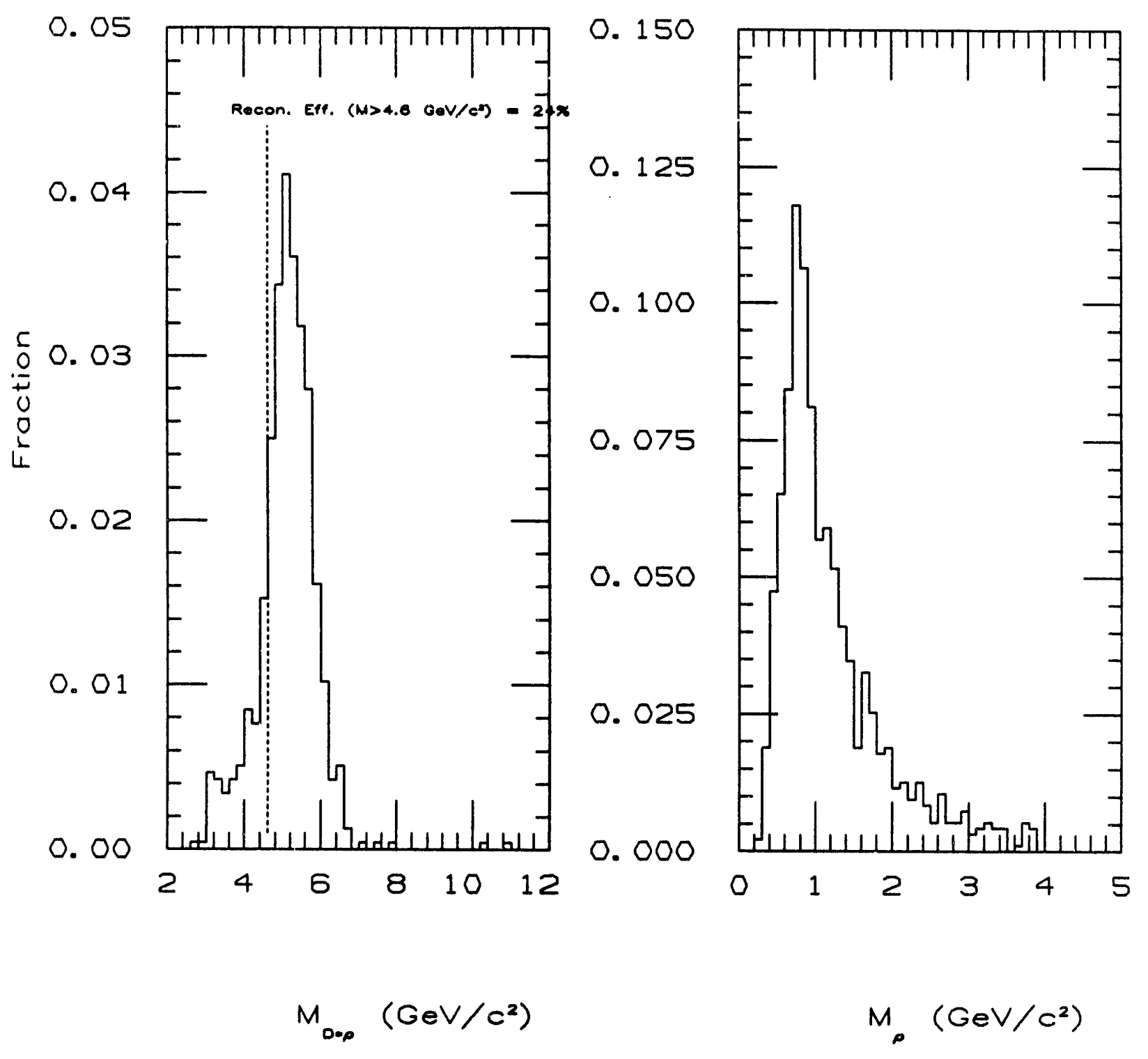

Figure 24: Testing $D^{*}$ trick for a $D^{*} \rho$ jet sample: (a) Mass distribution of $D^{*} \rho$ system using $D^{*}$ trick. About $24 \%$ of jets in the sample is reconstructed with $M>4.6 \mathrm{GeV} / \mathrm{c}^{2}$. (b) Mass distribution of $\rho\left(\pi_{f a s t} \pi^{0}\right)$ system. 

(a) $D^{*}+\pi_{\text {roat }}+E M$ cluster
(b) $\pi_{\text {ront }}+E M$ cluster
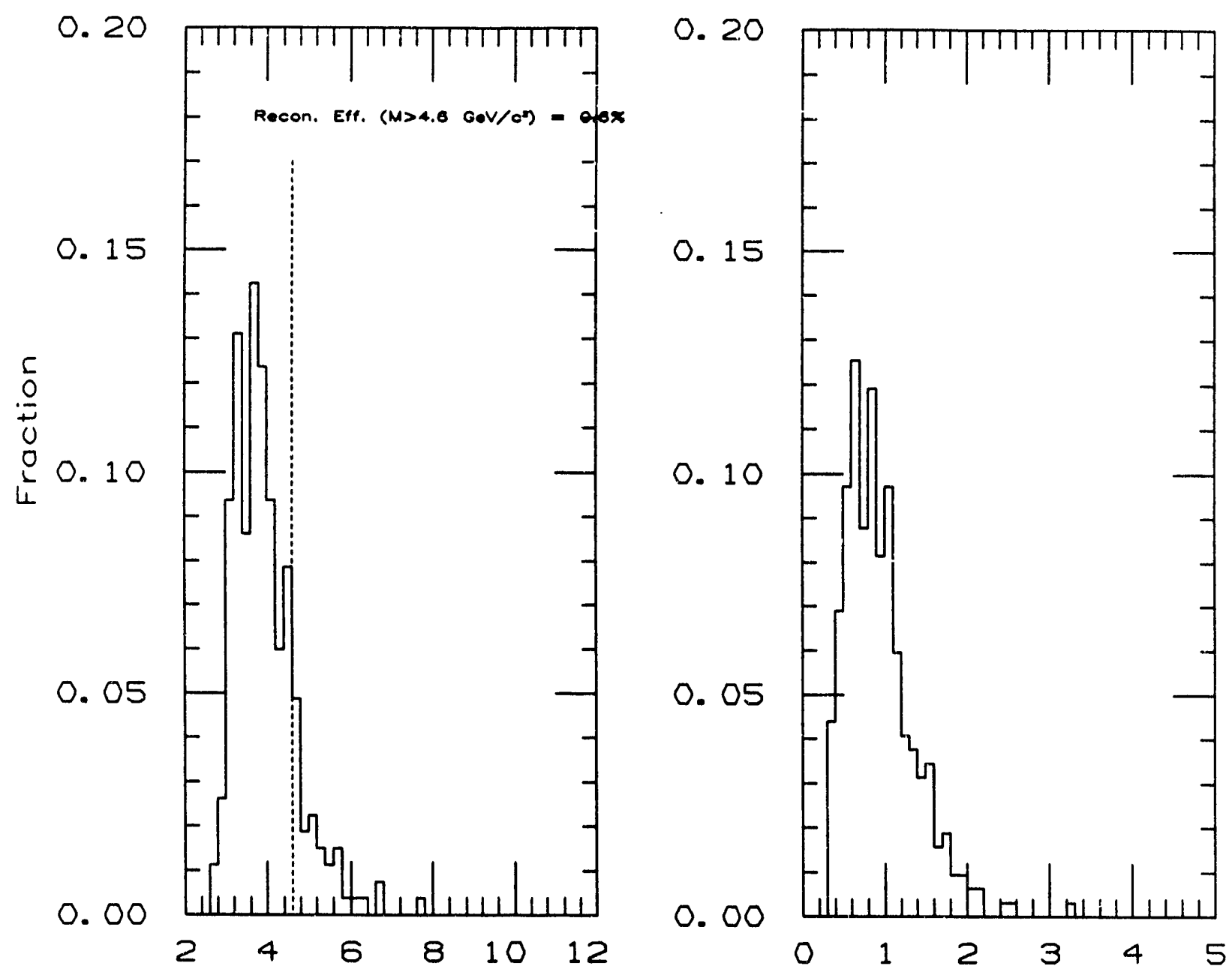

$$
M_{\text {Dथp }}\left(\mathrm{GeV} / \mathrm{c}^{2}\right)
$$

$M_{p}\left(\mathrm{GeV} / \mathrm{c}^{2}\right)$

Figure 25: T'esting $D^{*}$ trick for a $u / d$ jet sample: (a) Mass distribution of $D^{*} \rho$ system using $D^{*}$ trick. About $0.6 \%$ of jets in the sample is reconstructed to be consist with $D^{*} \rho$ jet $(M>4.6$ $\left.\mathrm{GeV} / \mathrm{c}^{2}\right)$. (b) Mass distribution of $\rho\left(\pi_{f a s t} \pi^{0}\right)$ system. 


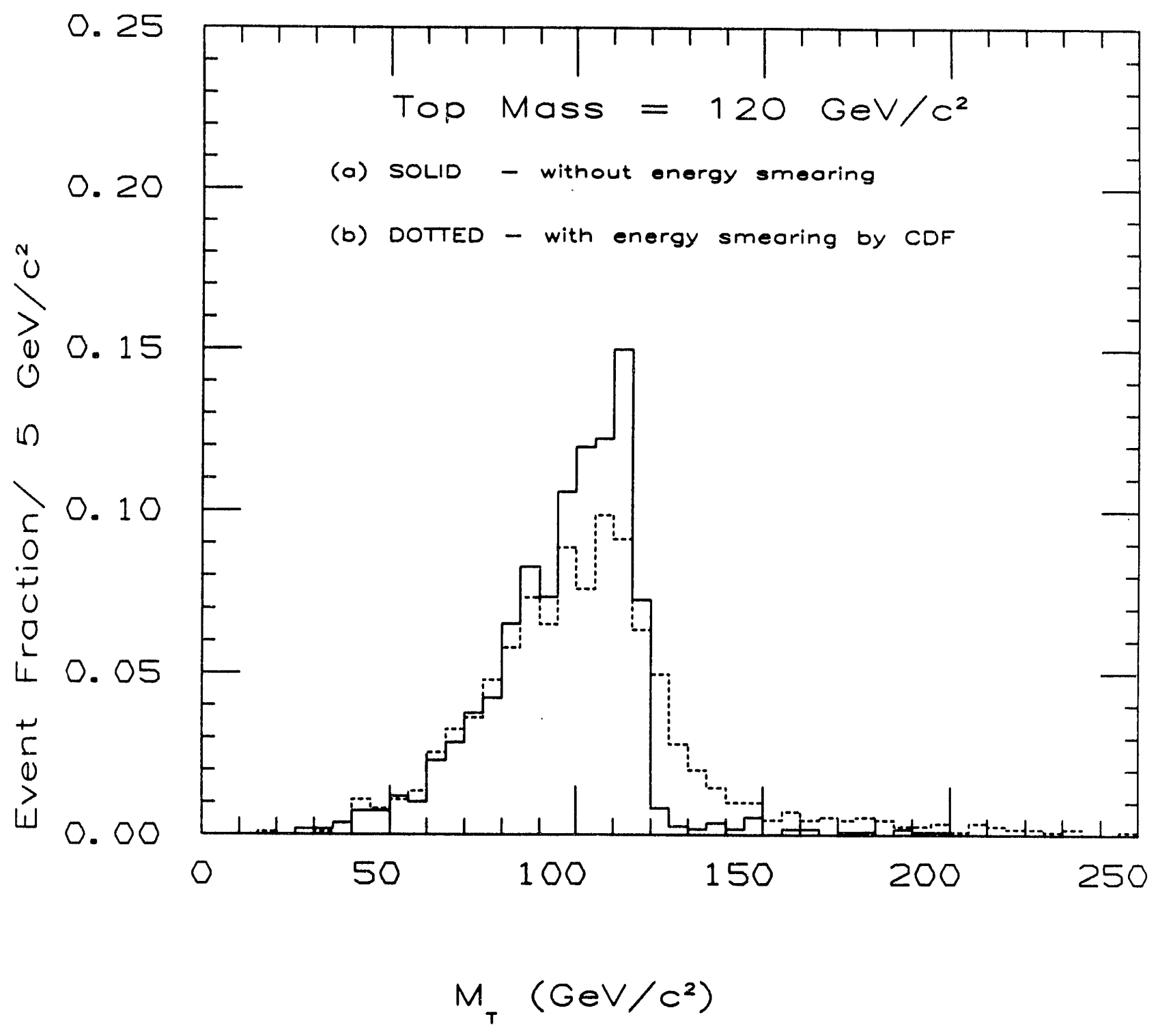

Figure 26: Monte Carlo simulation for $120 \mathrm{GeV} / \mathrm{c}^{2}$ top quark: (a) Transverse mass distribution of $\ell-\nu-B$ system without the energy resolution smearing. (b) Transverse mass distribution of $\ell-\nu \cdot B$ system with the energy resolution smearing by CDF. 

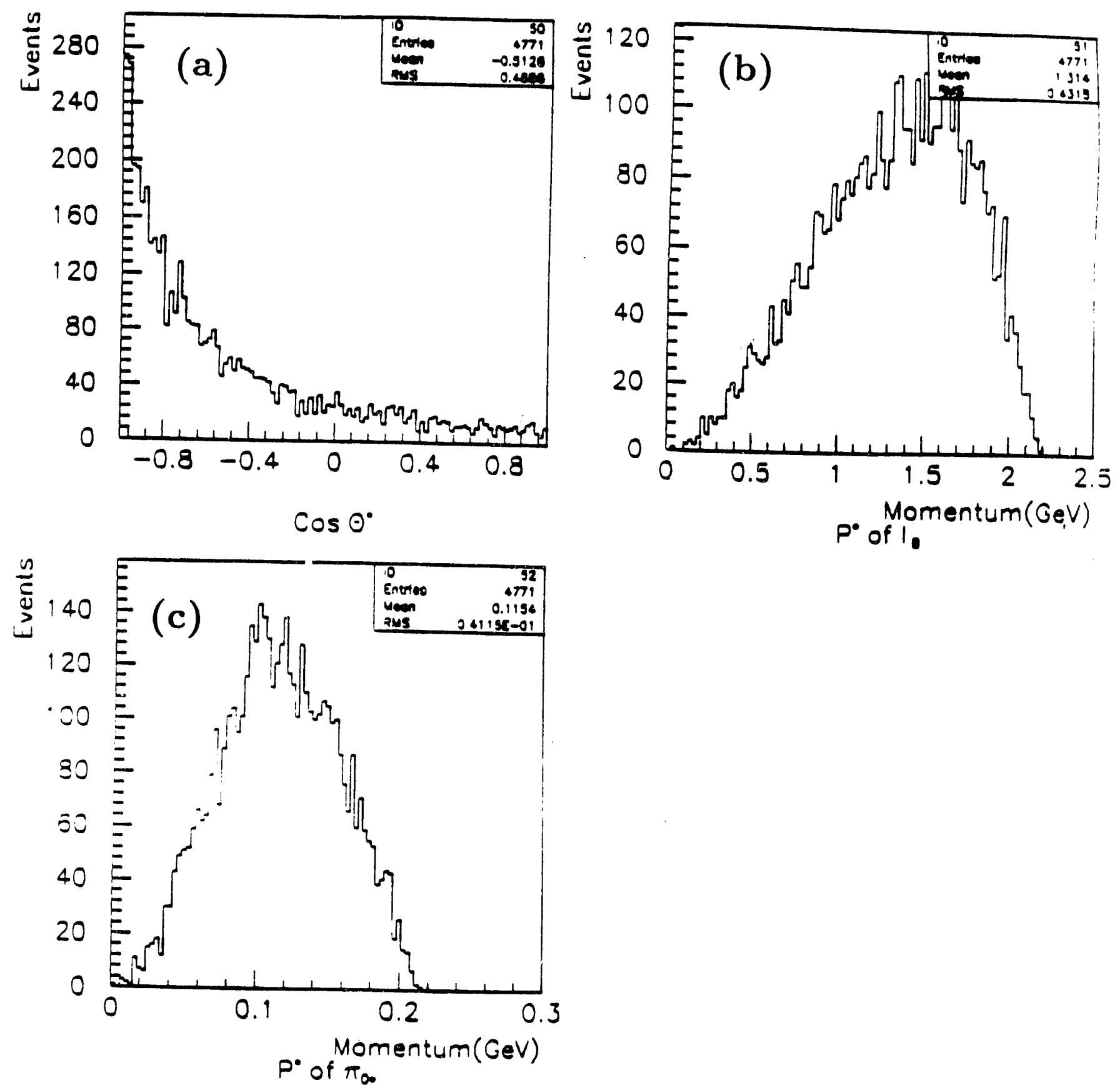

Figure 27: Distributions of kinematic variables in the rest frame of $B$ meson decaying into $D^{*} \ell \nu$ and $D^{*} \rightarrow D \pi:$ (a) $\cos \theta^{*}(\ell-\pi),\left(\right.$ b) $P^{*}\left(\ell_{B}\right)$ and (c) $P^{*}\left(\pi_{D^{*}}\right)$ for the M.C. sample $\left(M_{\text {top }}=120\right.$ $\left.\mathrm{GeV} / \mathrm{c}^{2}\right)$. 


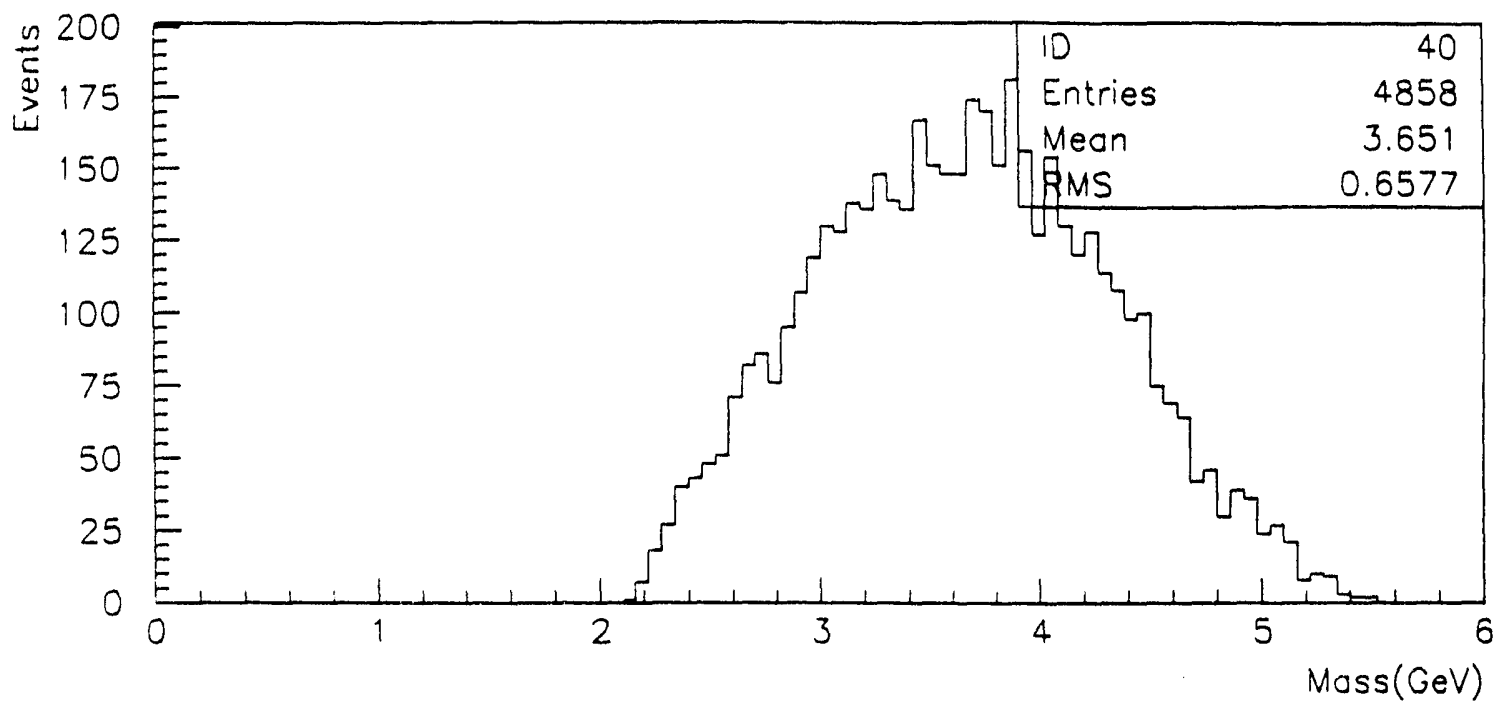

Figure 28: Partially-reconstructed mass distribution of $B$ mesons in the M.C. sample $\left(M_{\text {top }}=120\right.$ $\left.\mathrm{GeV} / \mathrm{c}^{2}\right)$ without cuts on $P^{*}$ and $\cos \theta^{*}$. 


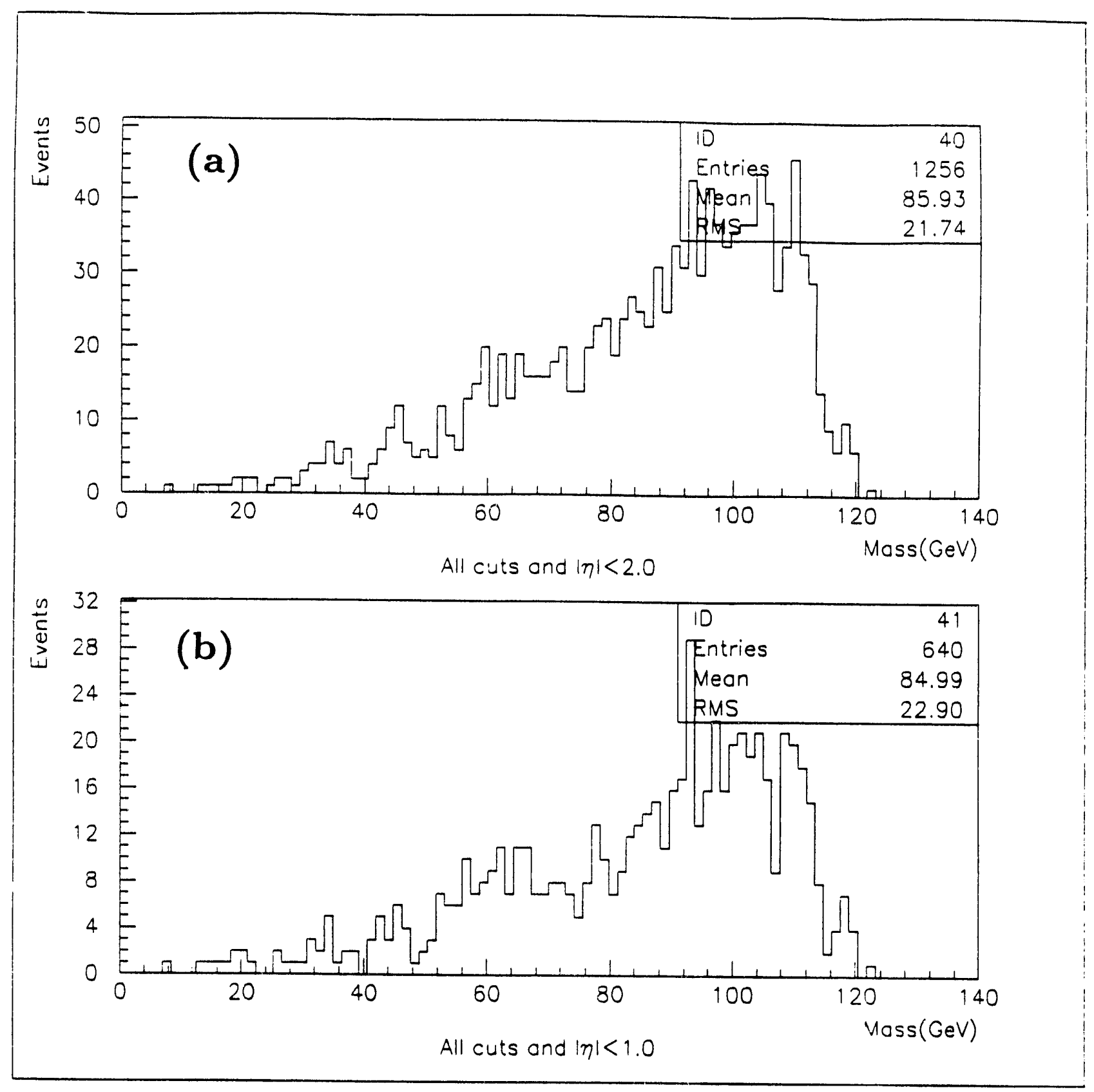

Figure 29: Reconstructed top quark mass distribution for $M_{\text {top }}=120 \mathrm{GeV} / \mathrm{c}^{2}$ with cuts on $P_{T}\left(\ell_{W}\right)$, $P_{T}\left(\ell_{B}\right), P_{T}\left(\pi_{D^{*}}\right), P_{\ell}^{*}, P_{\pi}^{*}$ and $\cos \theta^{*}:$ (a) $|\eta|<2$, (b) $|\eta|<1$. 


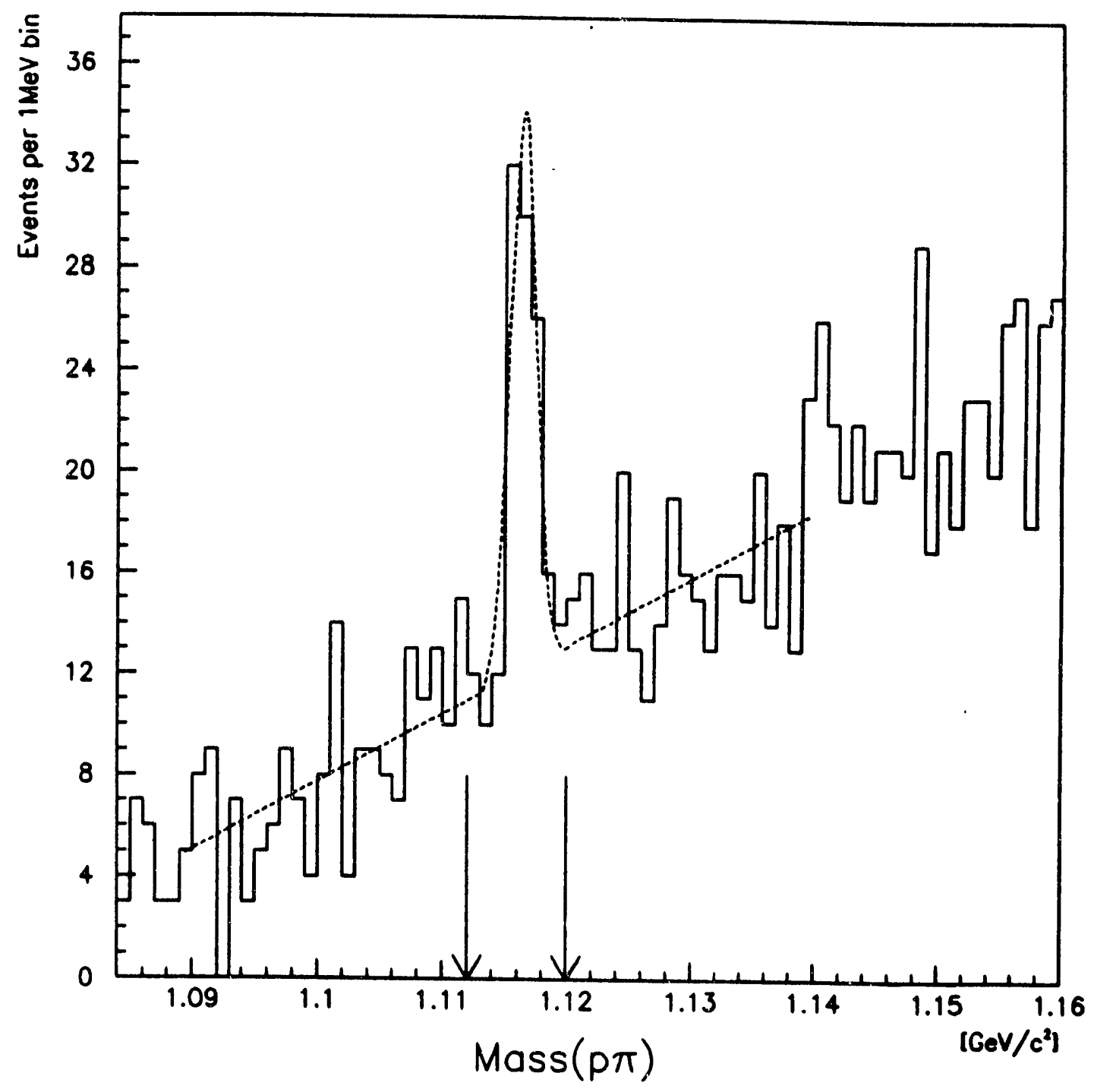

Figure 30: $p-\pi$ invariant mass spectrum from the $1988-89$ data. The $52 \pm 12 \Lambda^{0}$ signals were found. 


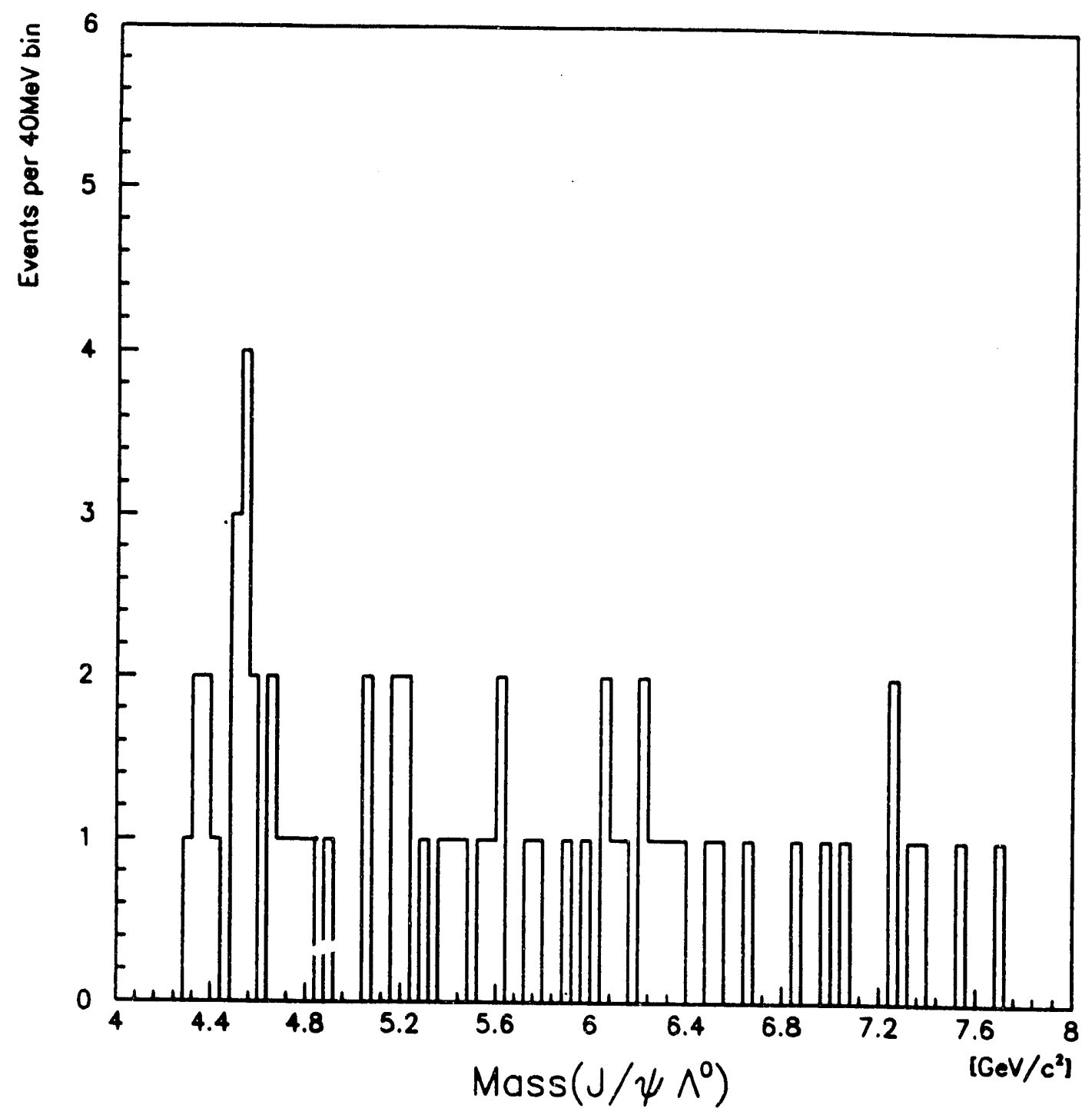

Figure 31: $\Lambda^{0} \mathrm{~J} / \psi$ invariant mass spectrum from the $1988-89$ data. The background level is $0.7 \pm 0.2$ events per $40 \mathrm{MeV} / \mathrm{c}^{2}$ bin in the region between $5300 \mathrm{MeV} / \mathrm{c}^{2}$ and $6000 \mathrm{MeV} / \mathrm{c}^{2}$. 


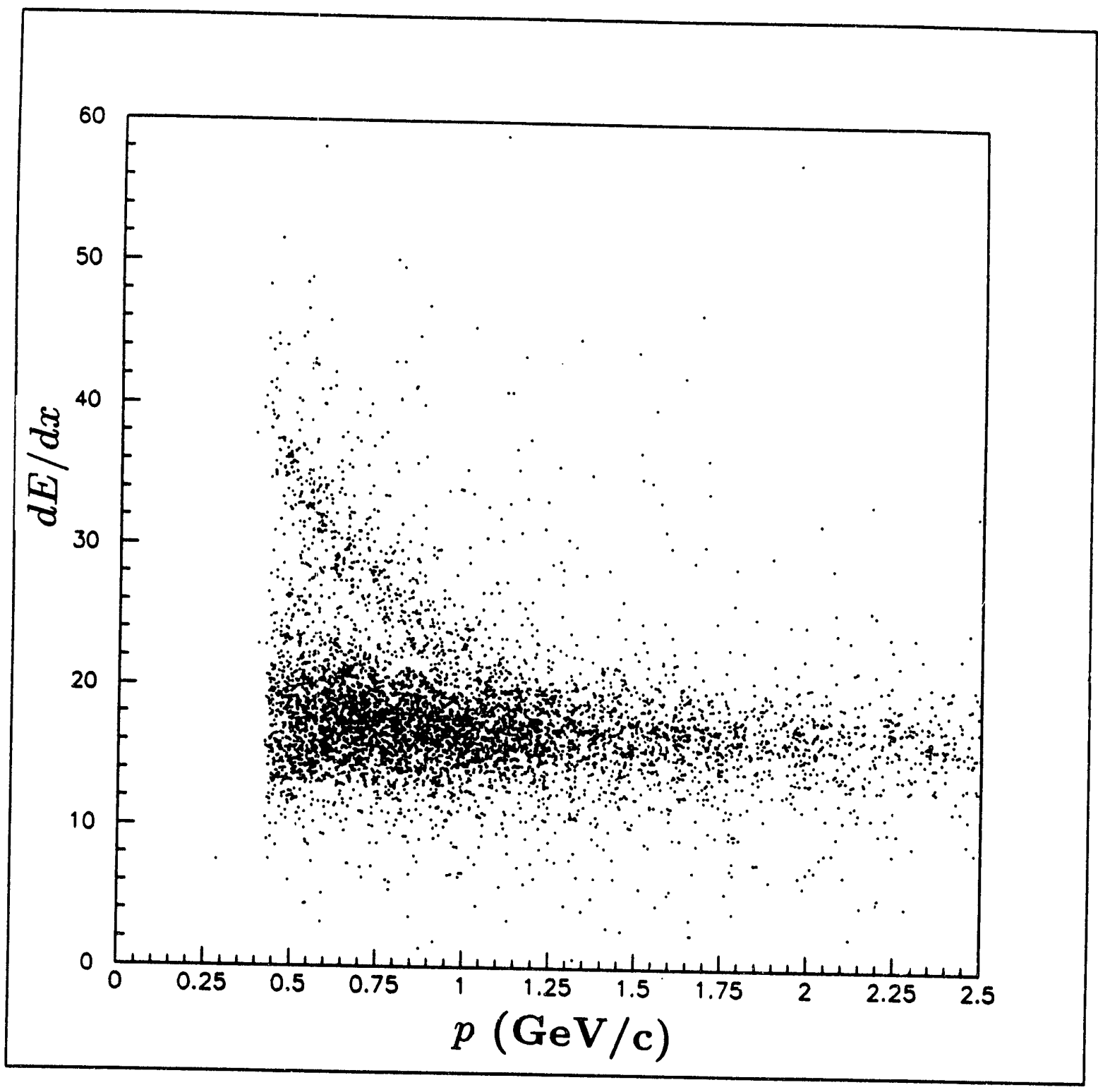

Figure 32: Preliminary plot of $d E / d x$ vs $p$ for tracks from minimum bias events. The $d E / d x$ values (in ADC counts) are without any calibration of the amplifier. The two bands are seen and expected to be $\pi$ and $K / p$. 

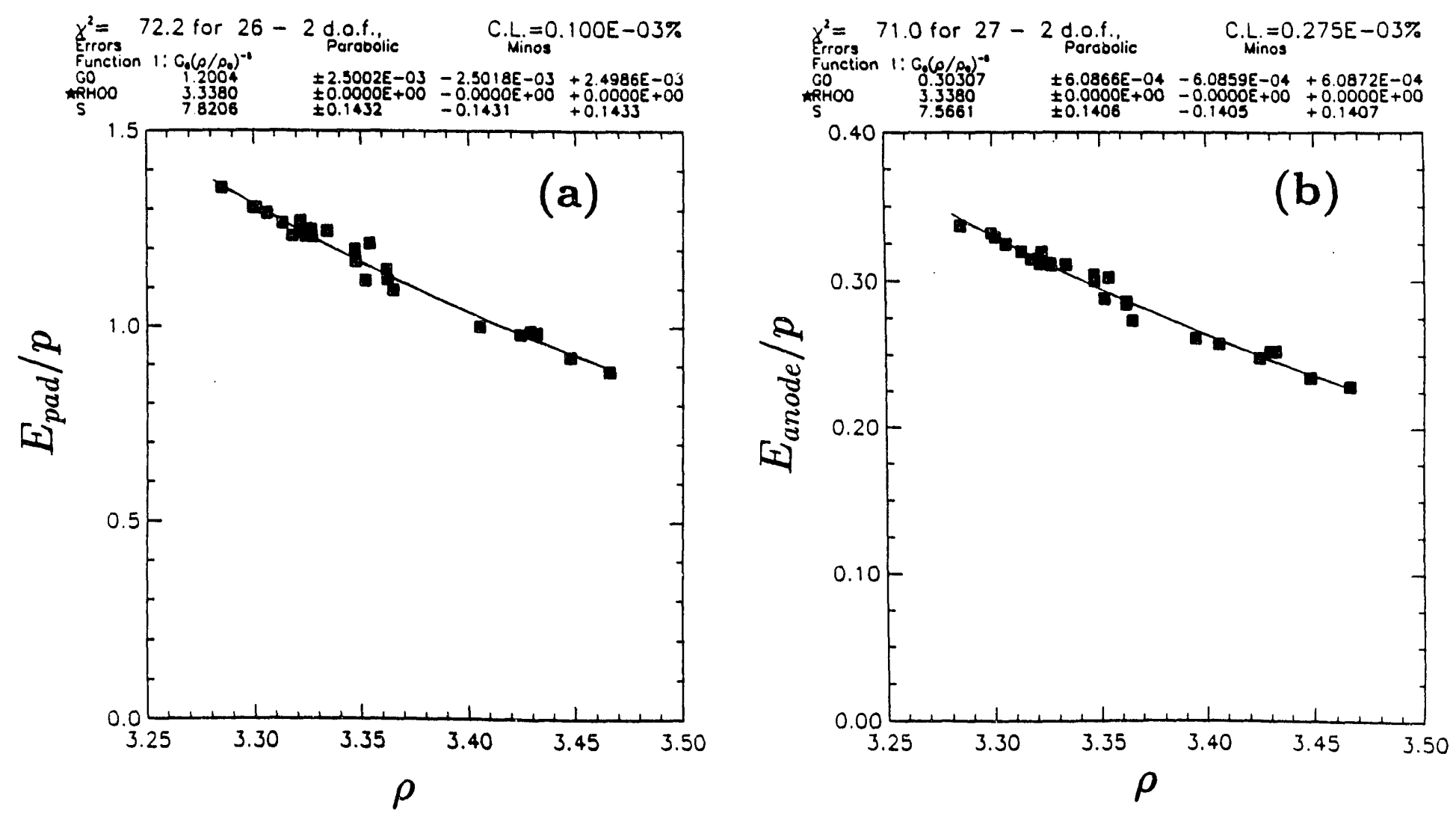

Figure 33: $E / p$ vs $\rho$ : (a) pad ant (b) anode energies. 


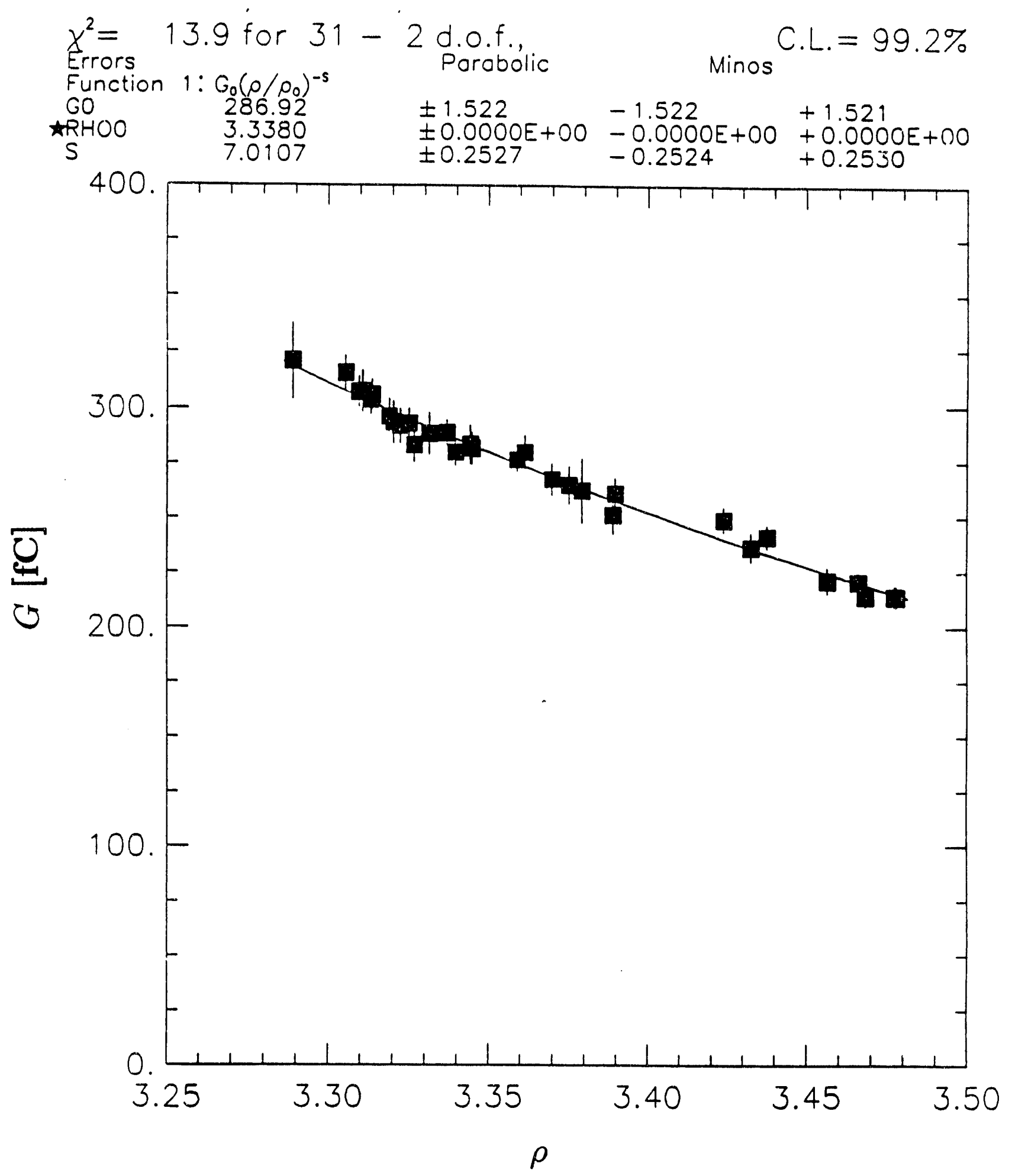

Figure 34: Gas gain vs $\rho$ for $O U T 1$ tube. 


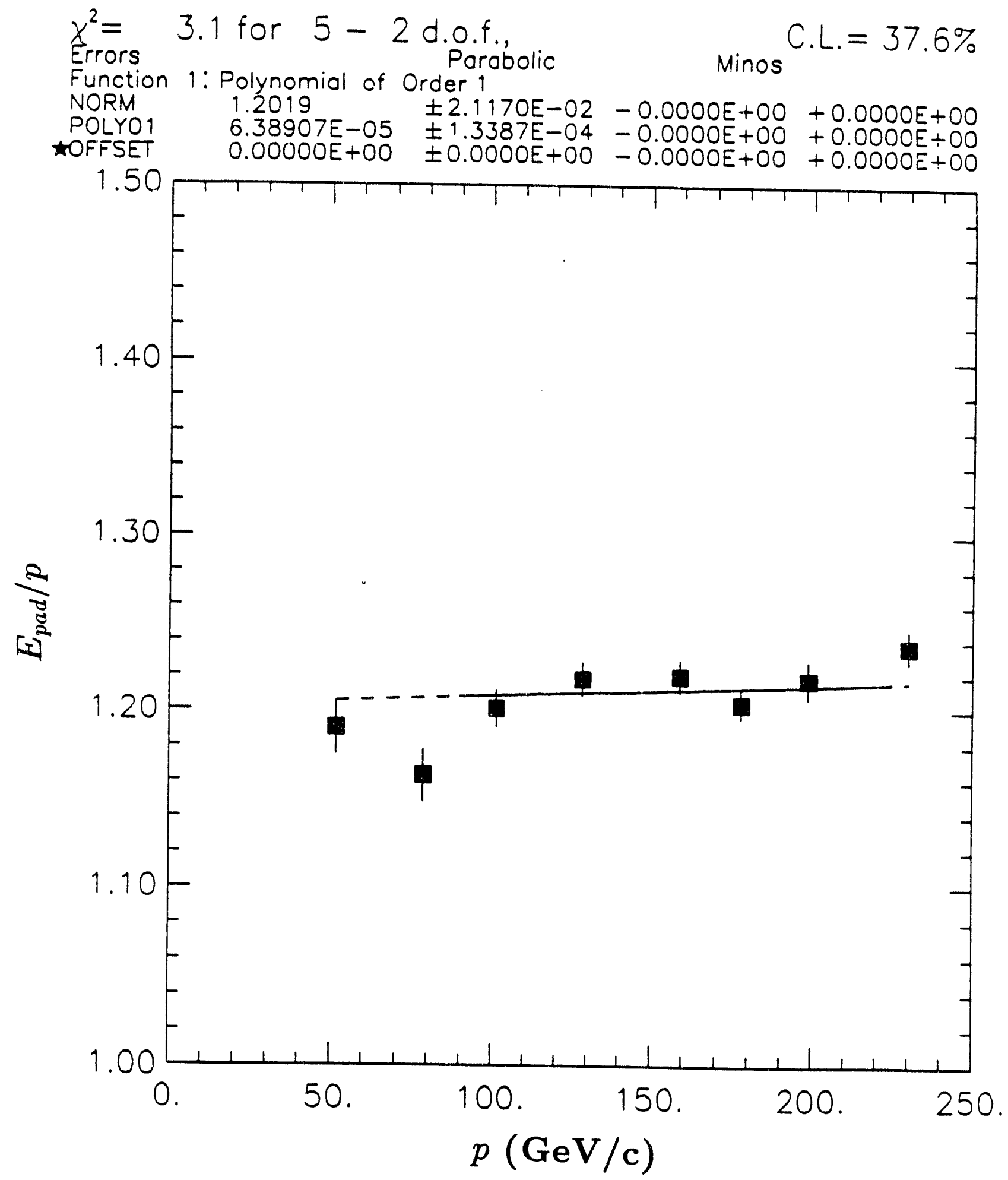

Figure 35: $E / p$ vs $p$. Some nonlinearity is observed in the lower momentum region. The solid line, fitted only between 100 and $200 \mathrm{GeV} / \mathrm{c}$, does not indicate nonlinearity. 


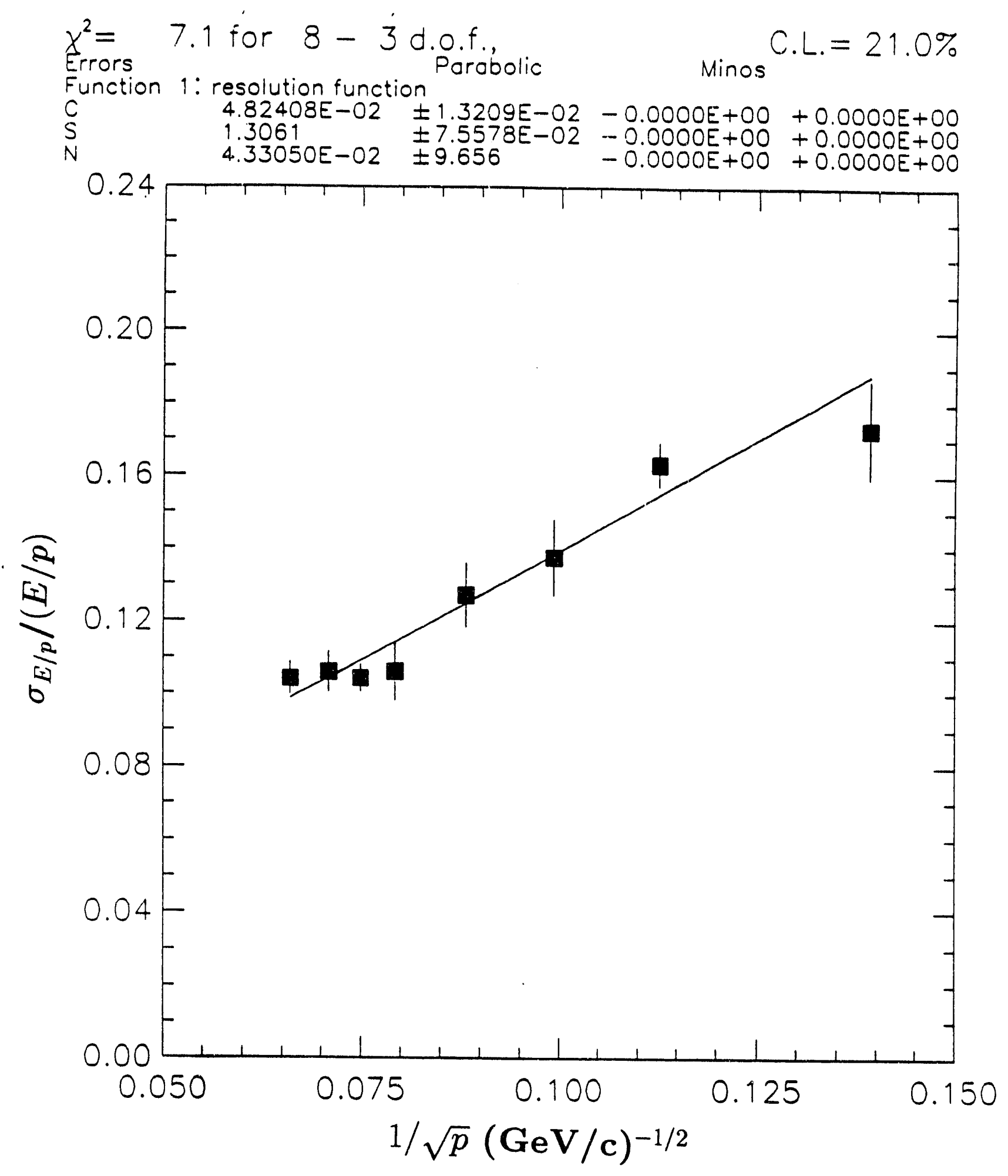

Figure 36: FHA energy resolution. 


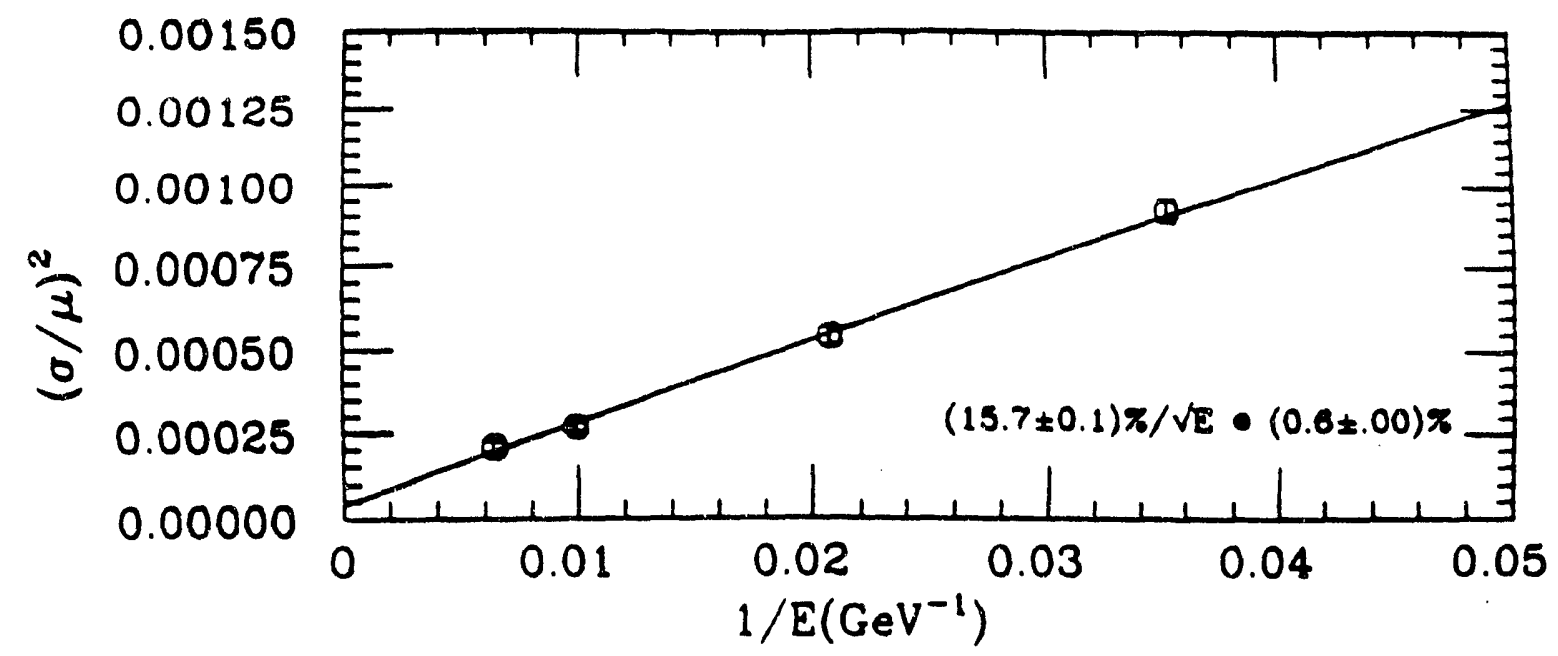

Figure 37: Testbeam: Energy resolution of plug electromagnetic calorimeter.

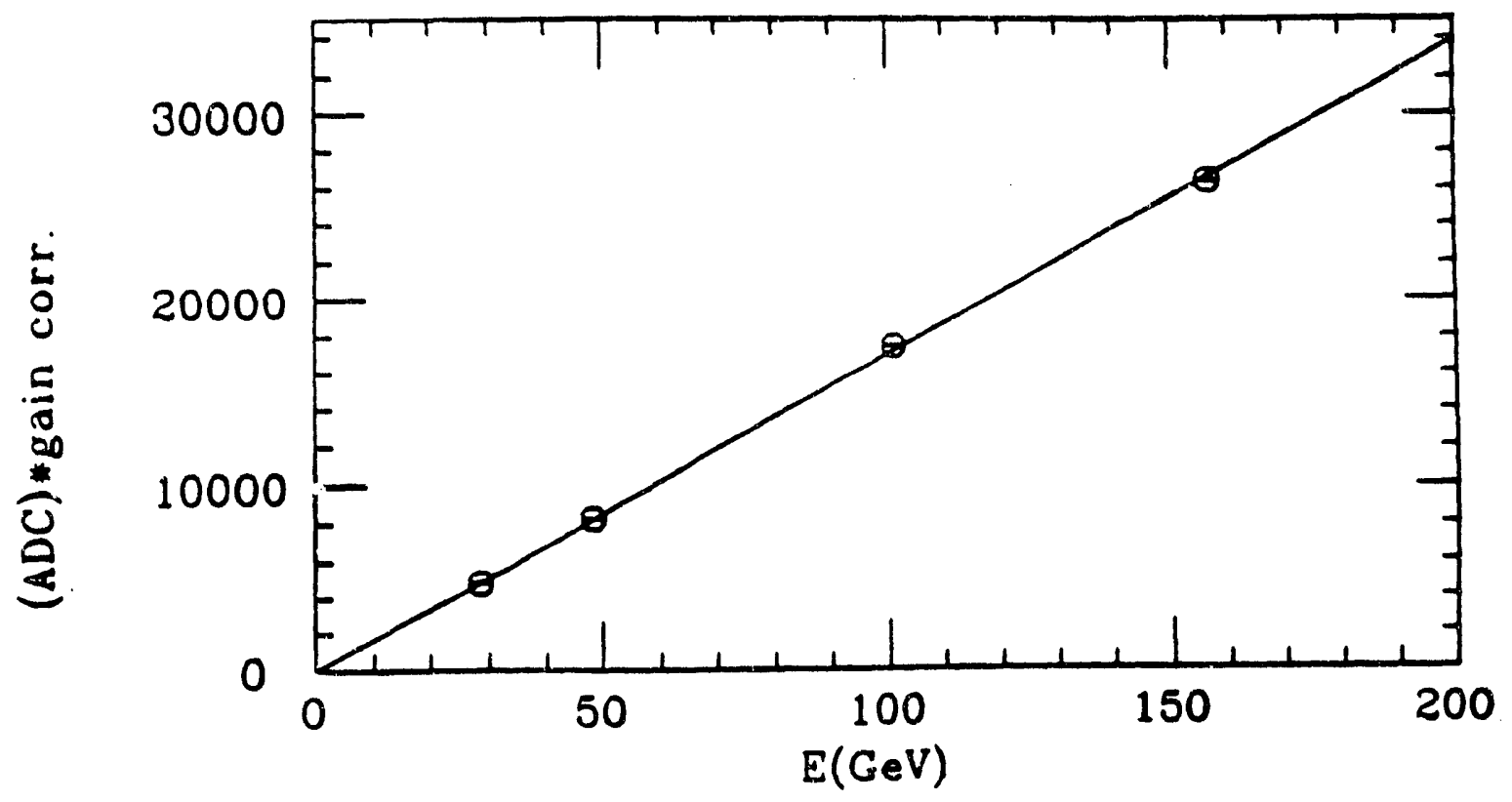

Figure 38: Testbeam: Linearity of plug electromagnetic calorimeter. 


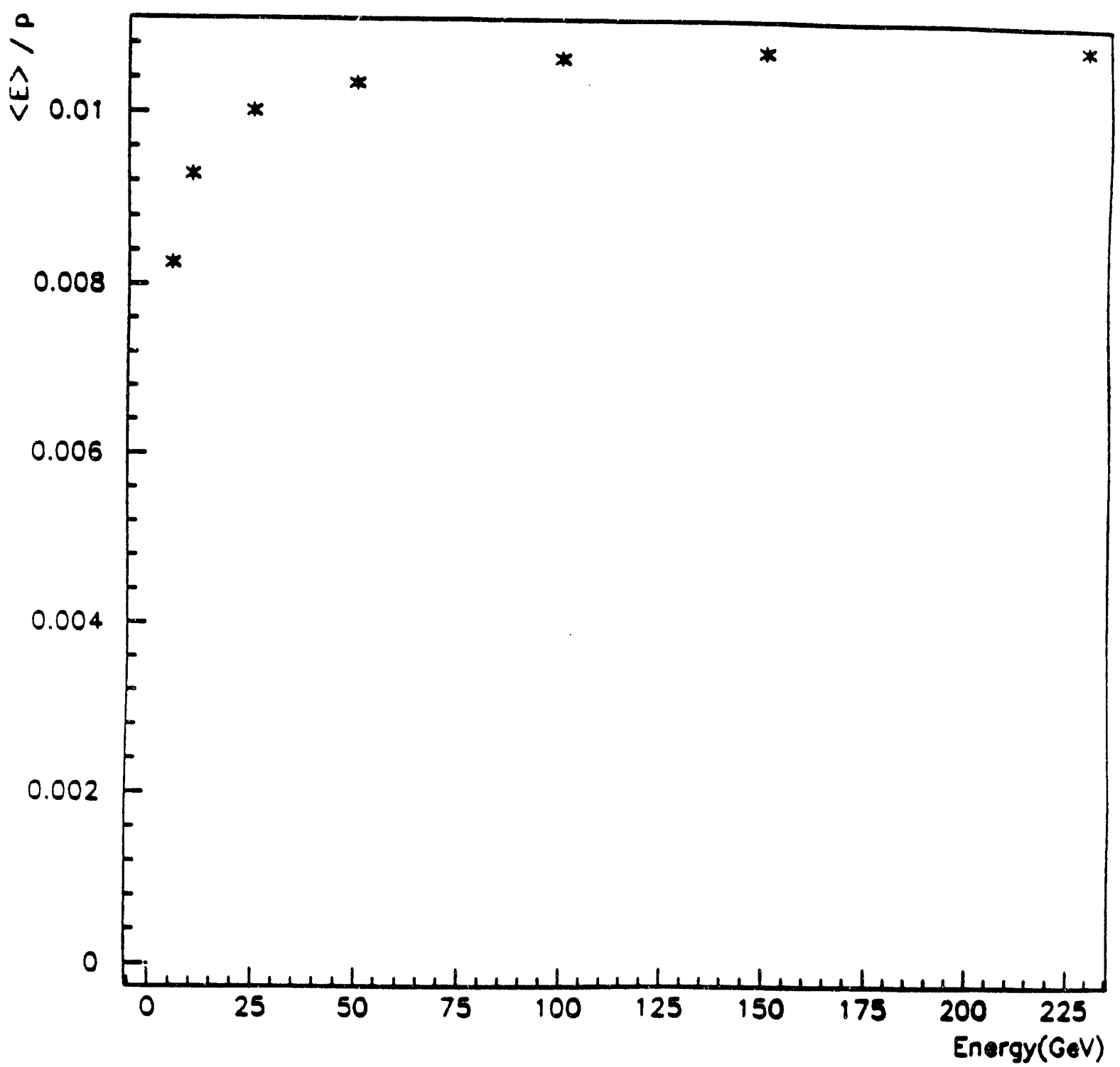

Figure 39: GEANT3: Linearity of plug hadron calorimeter: $\langle E\rangle / p$ (arbitrary units) vs $p$. 


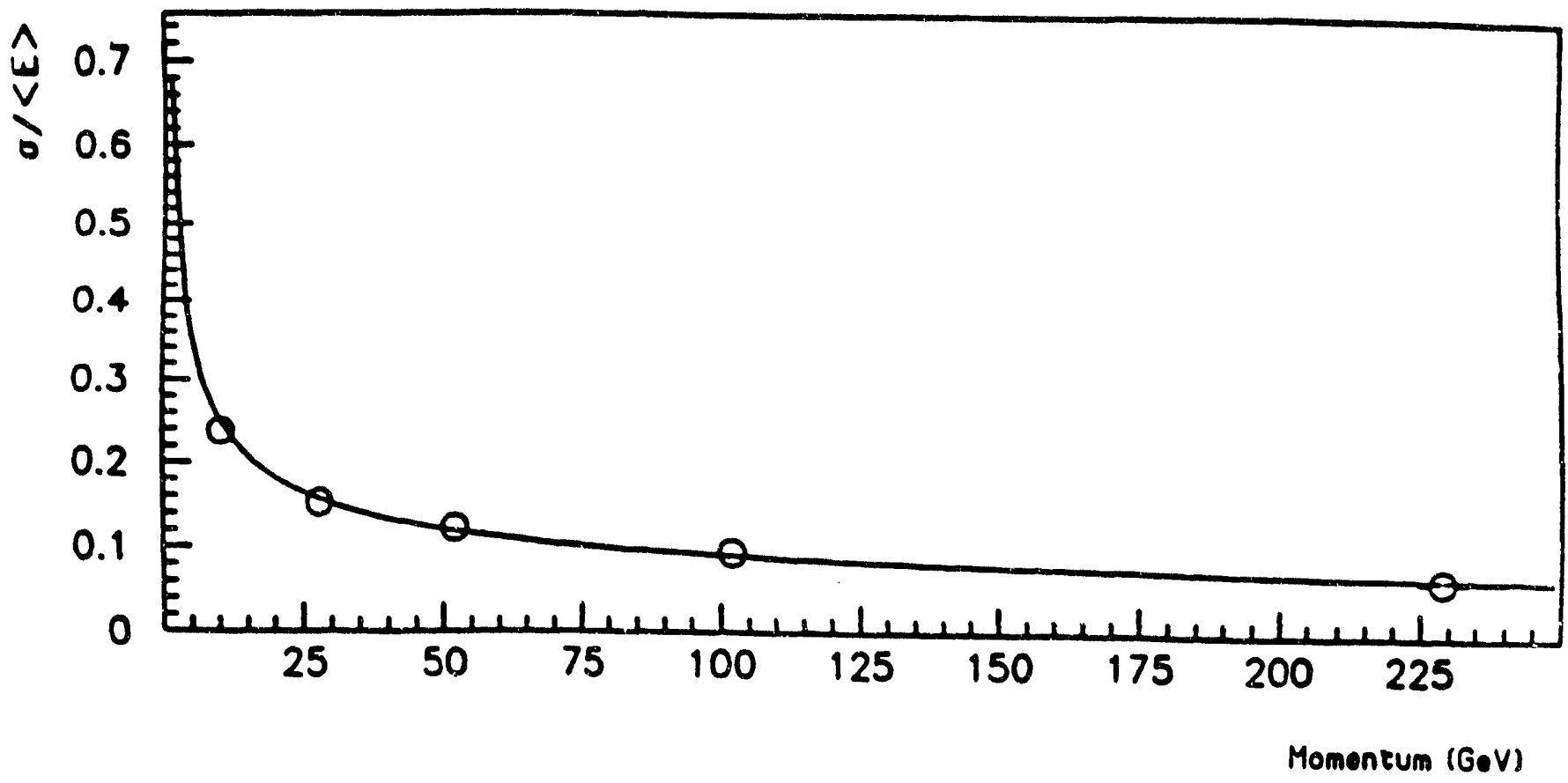

Figure 40: Testbeam: Energy resolution of plug hadron calorimeter.

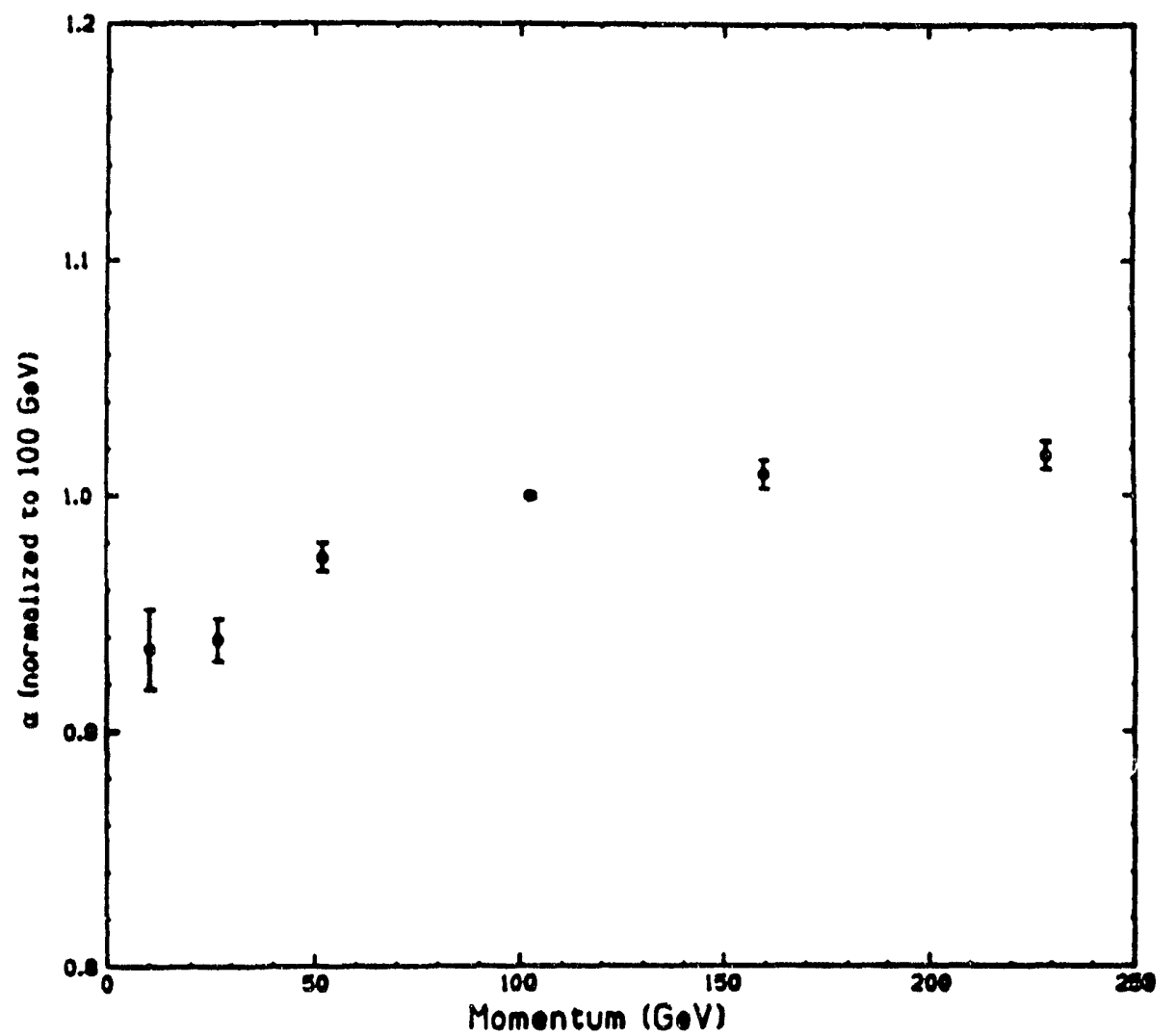

Figure 41: Testbeam: Linearity of plug hadron calorimeter. 


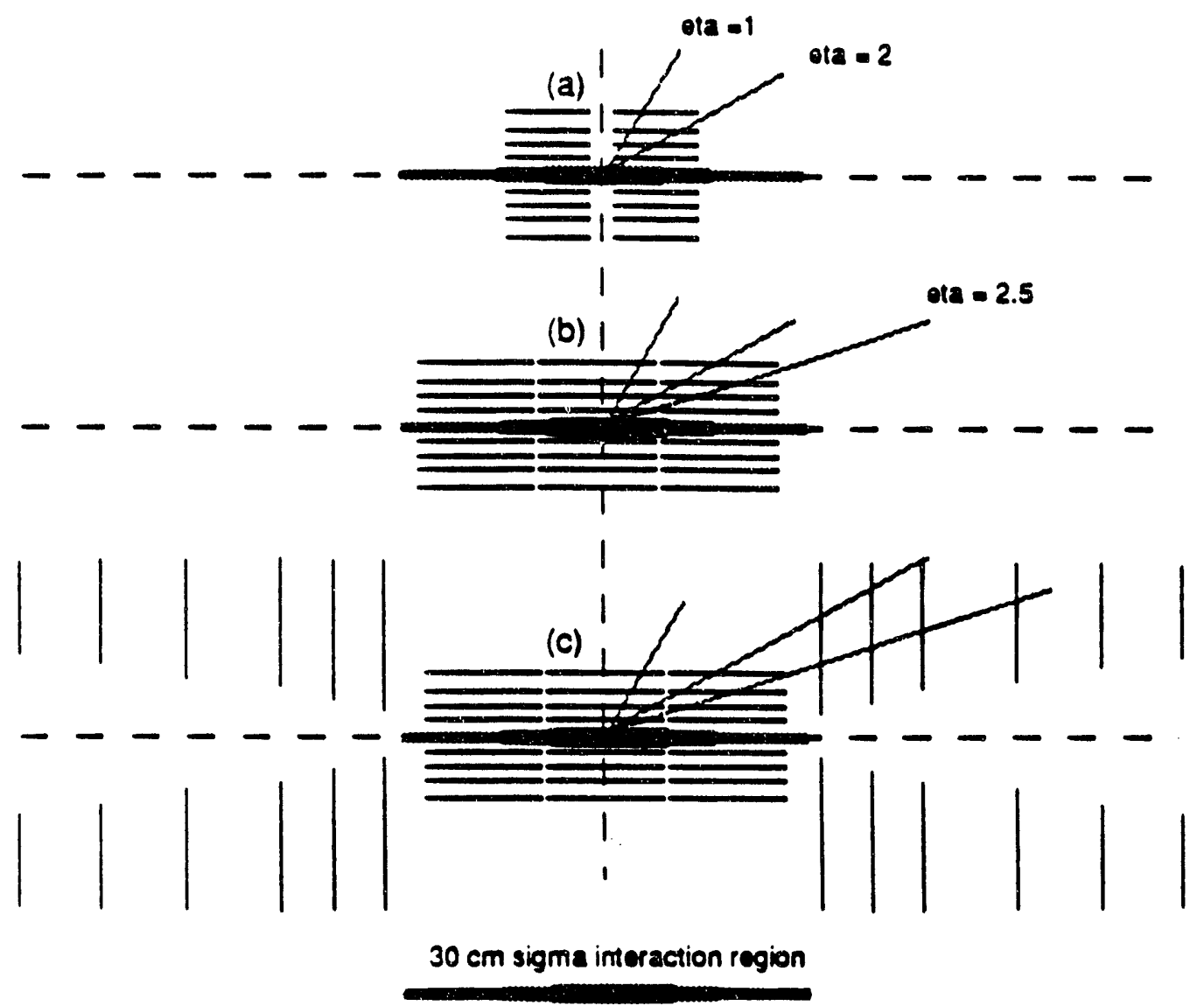

0.512 sigma

Figure 42: Schematic of the upgrade concept [46] showing: (a) The present SVX. Barrel geometry with a total length of $51 \mathrm{~cm}$. (b) SVX-II stage 1. Barrel geometry with a total length of $102 \mathrm{~cm}$. (c) SVX-II srage 2 as (b) with the addition of disk detectors for forward tracking. 
(a)

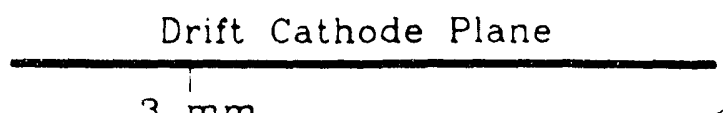

Metal 2

Al $-2 \mu \mathrm{m}$

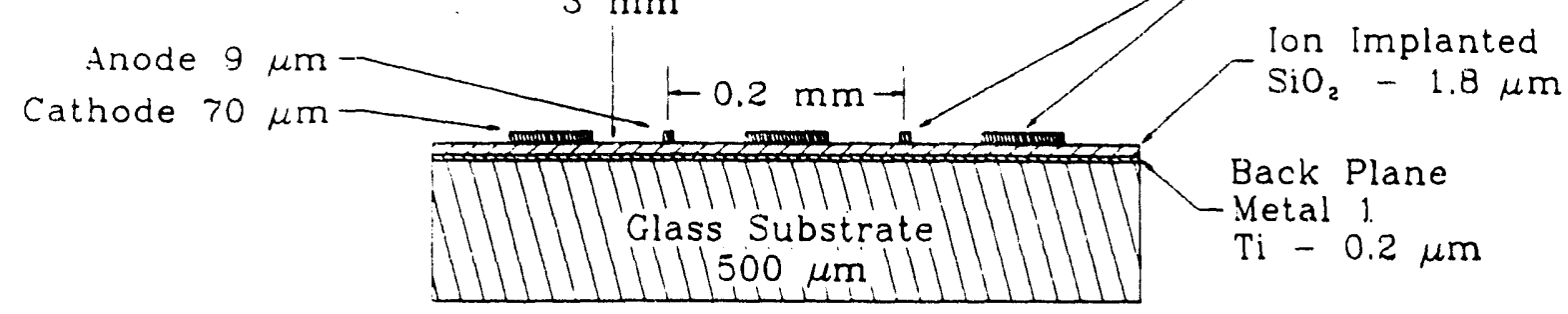

(b)

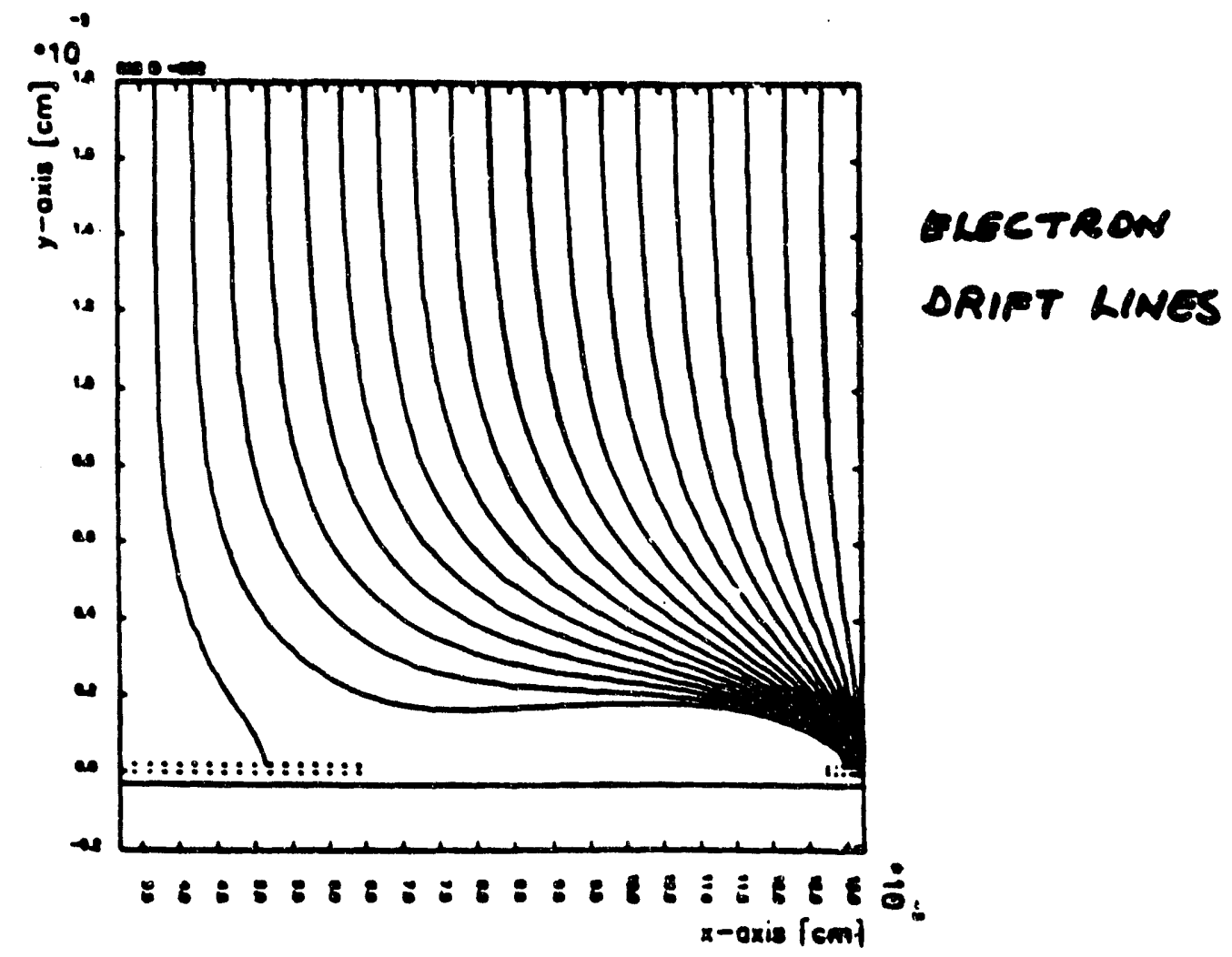

Figure 43: (a) Cross sectional view of two-dimensional readout gas microstrip chamber by Bellazzini's group [49] and (b) its field lines. 


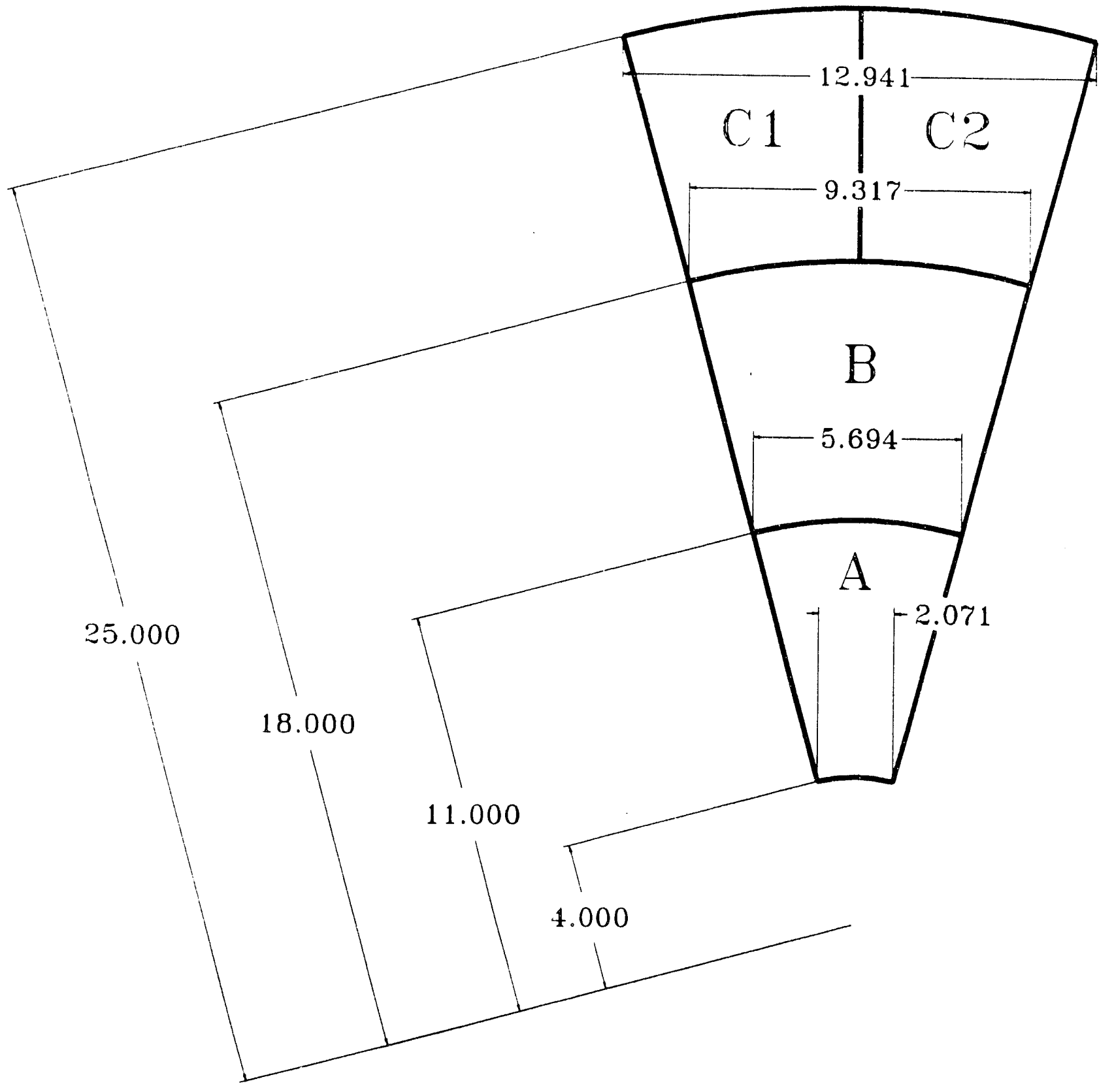

Figure 44: $30^{\circ}$-pie shape module consisting of 4 tiles (A, B, C1 and C2) with its demensions (unit: cm). 


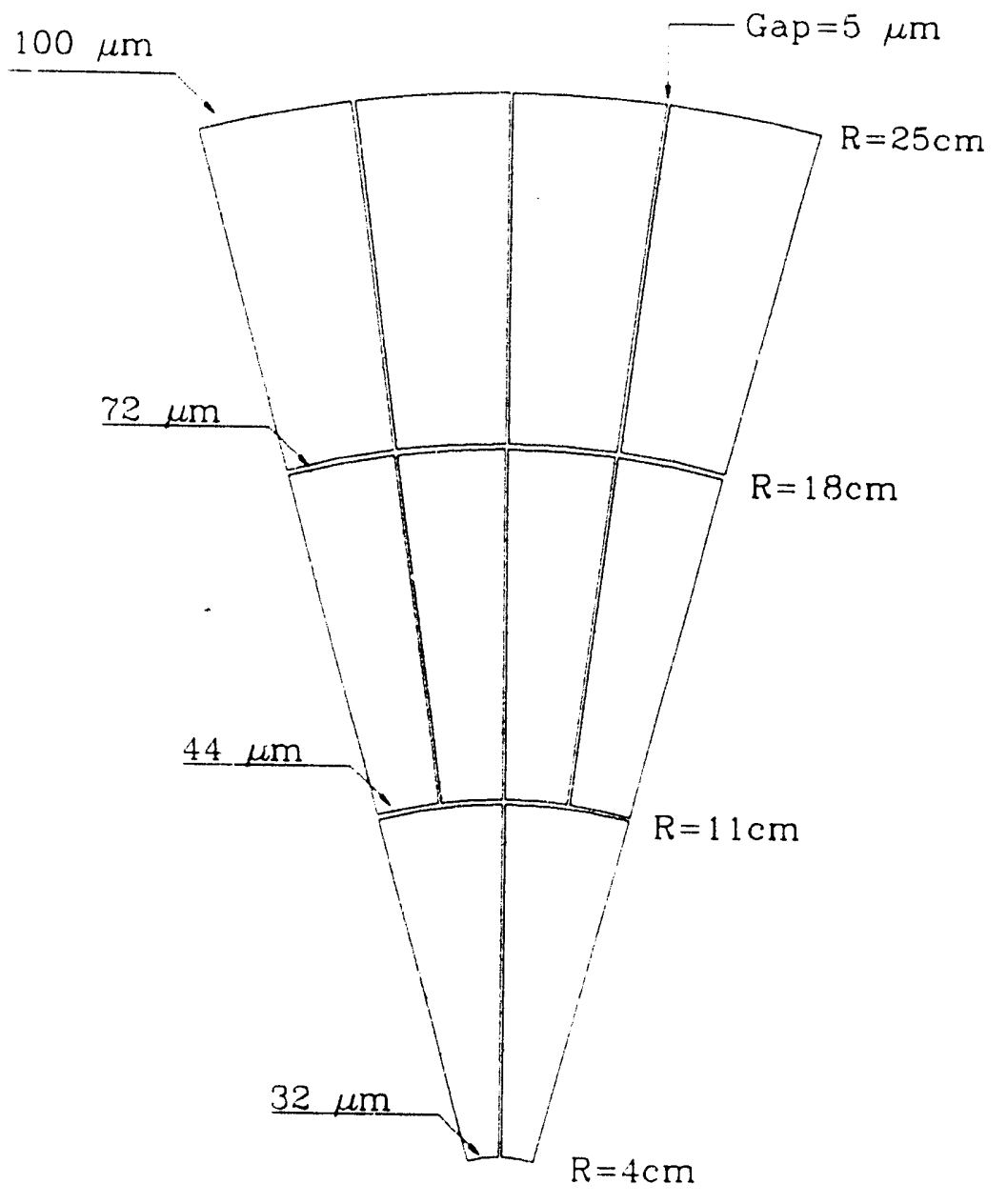

Figure 45: Backplane strip pattern showing the keystoned shapes. 


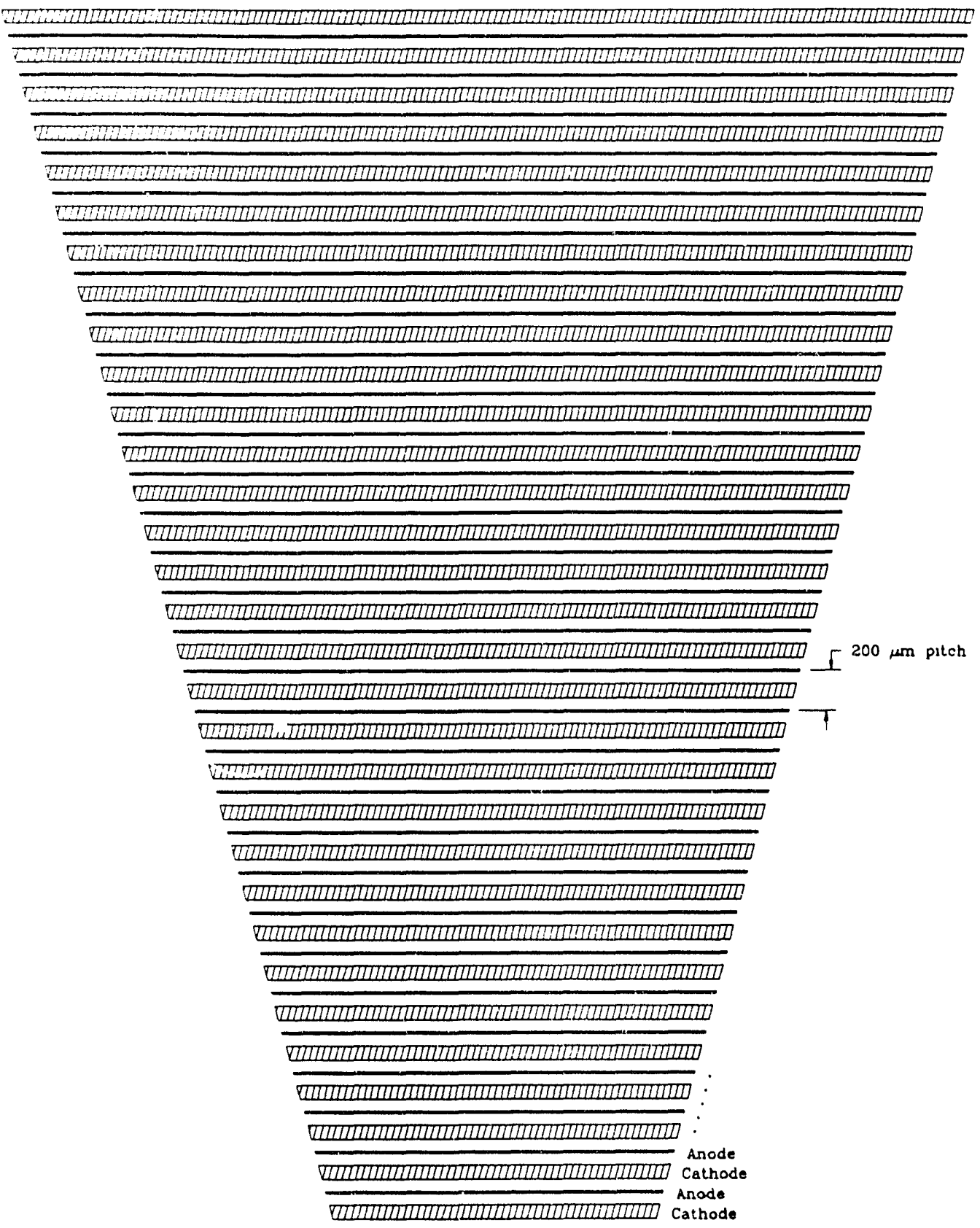

Figure 46: Anode/cathode strip pattern. 

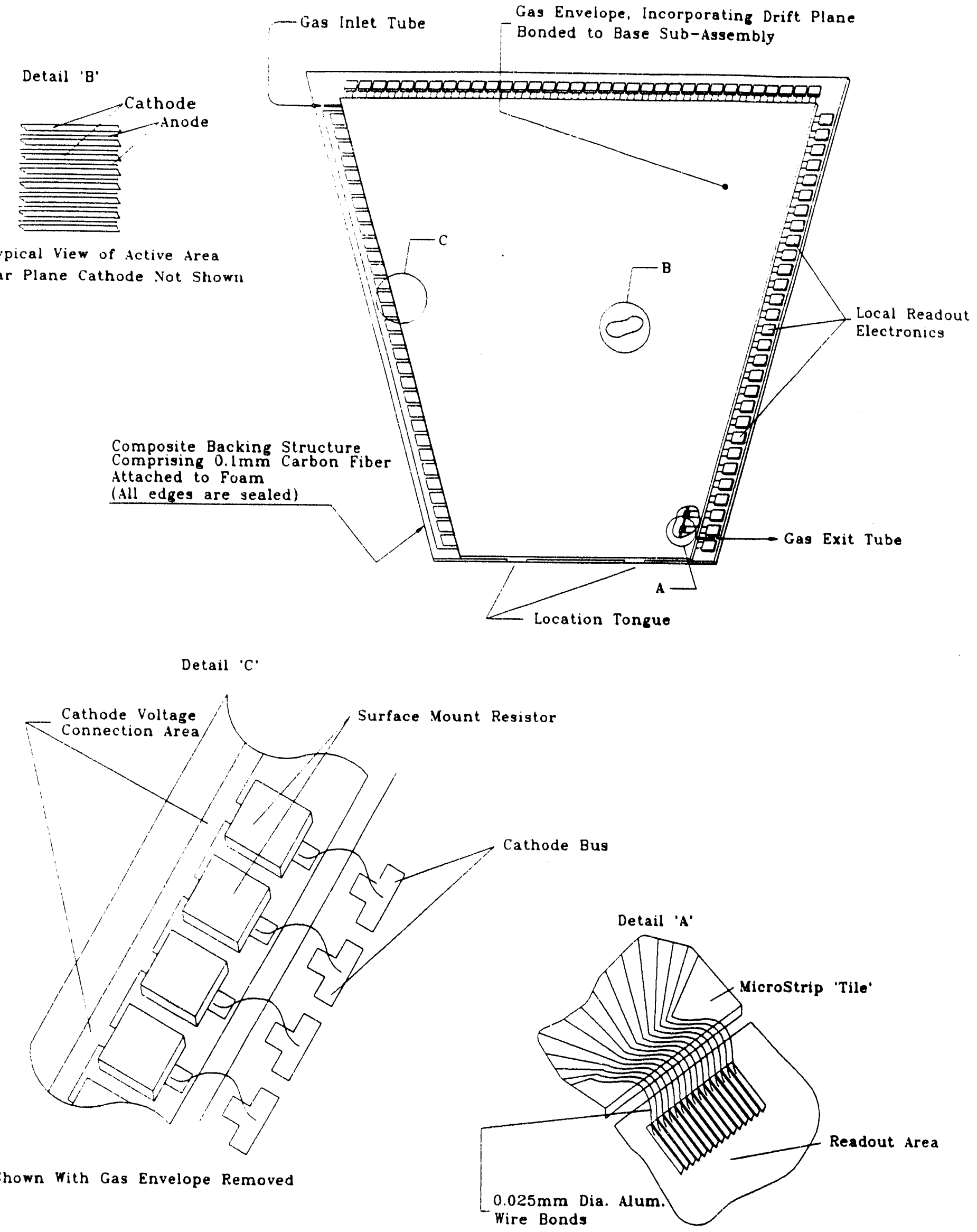

Figure 47: Schematic drawing of a tile. 


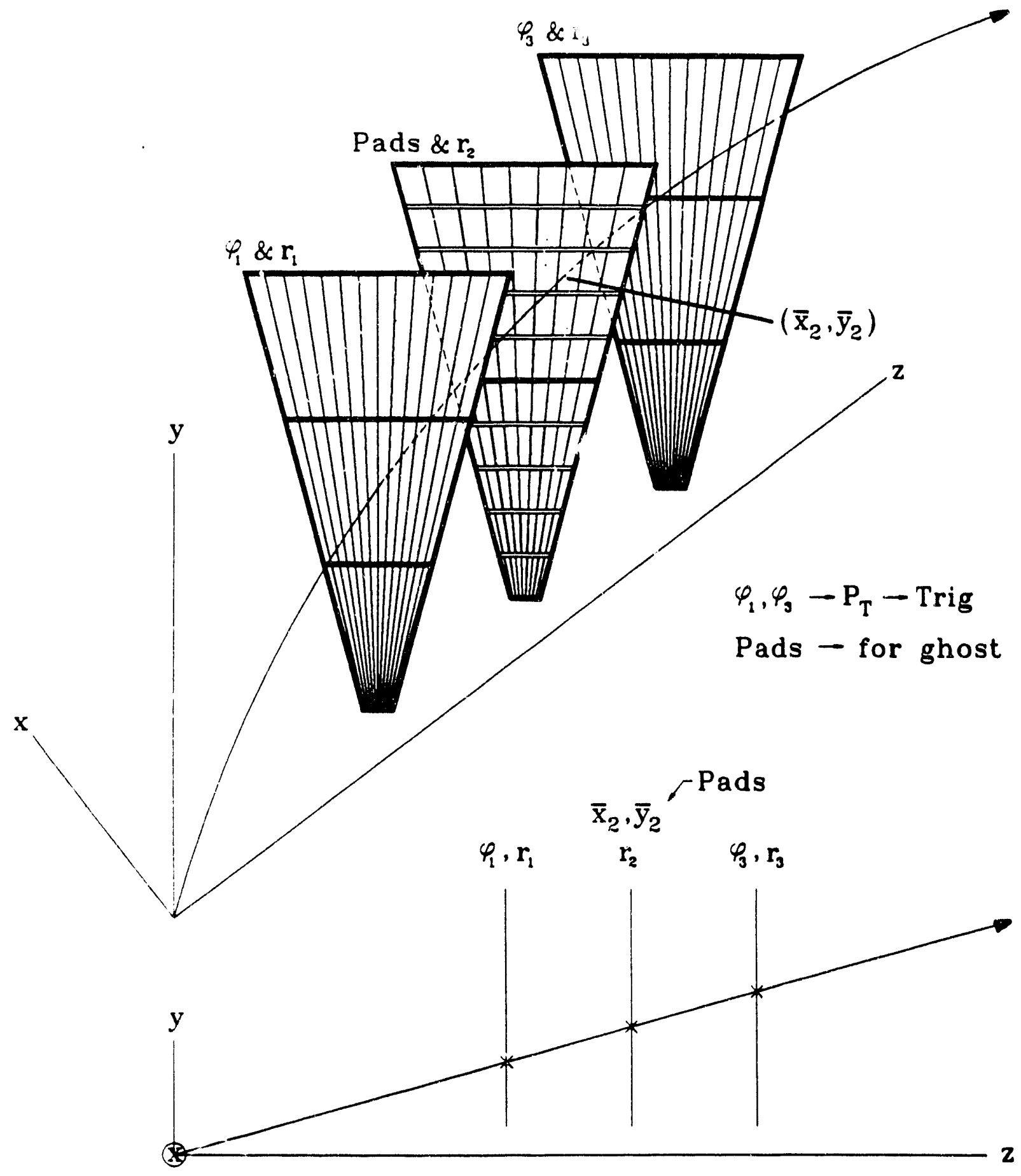

Figure 48: Possible configuration of keystoned radial strip and pad charobers. 
(a)

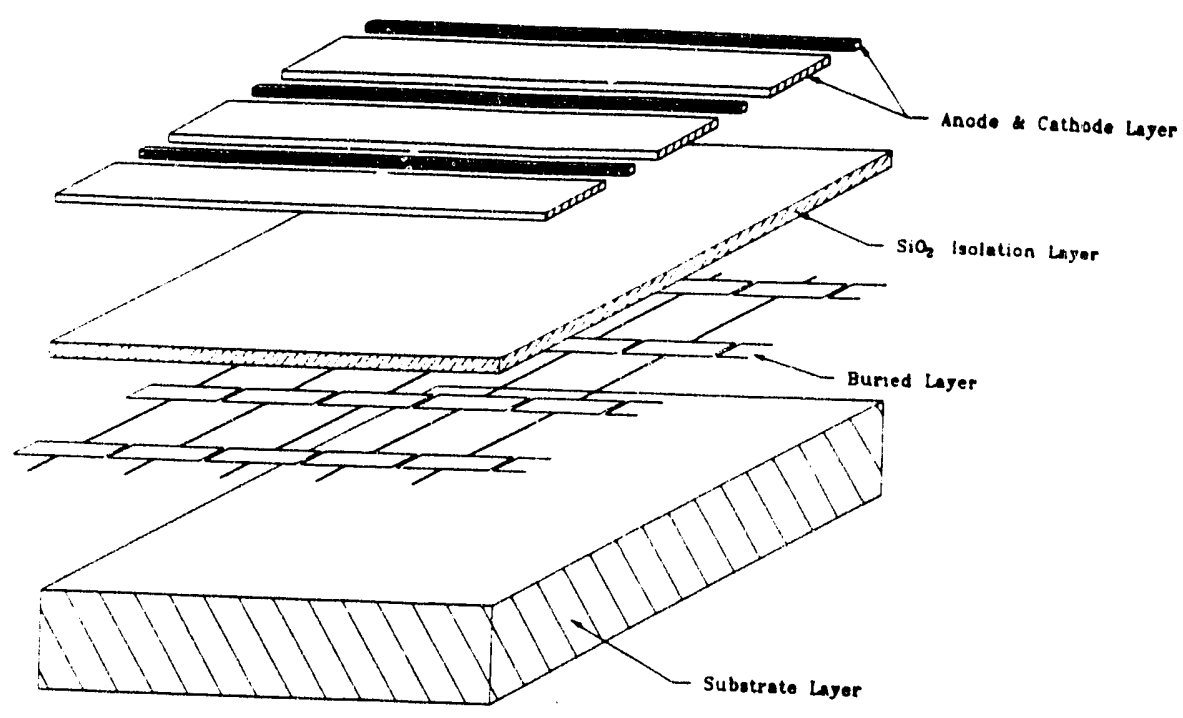

(b)

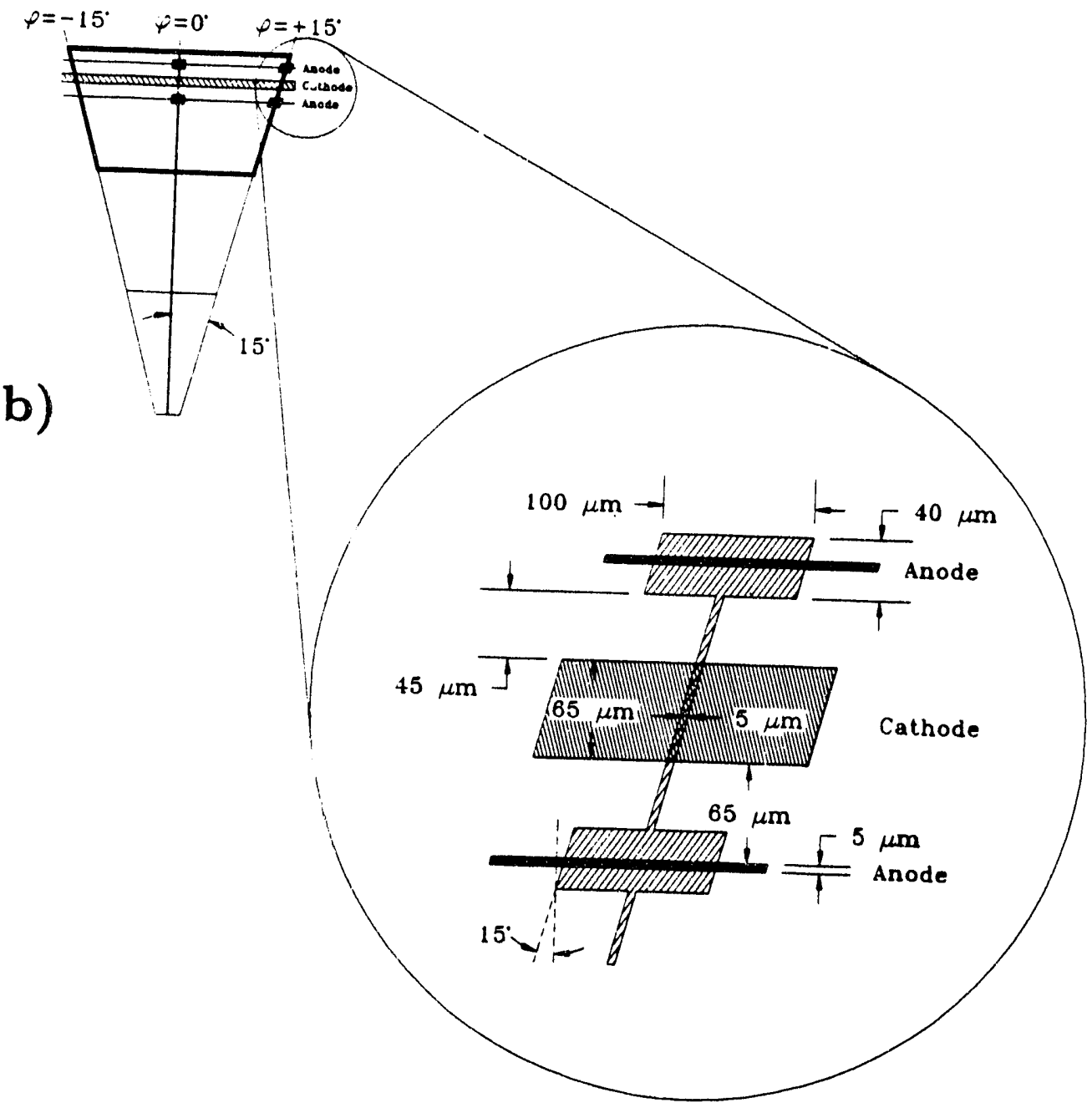

Figure 49: (a) Perspective view of two-dimensional readout gas microstrip chamber with new design of backplane strip ("buried layer") pattern; (b) Detailed design of the buried layer overlayed with anode/cathode strips at $\phi=15^{\circ}$. 


\section{The MACRO Experiment}

The MACRO experiment being staged at the Gran Sasso Laboratory in l'Aquila, Italy is the next generation underground, large surface area experiment designed to study magnetic monopoles, astrophysical neutrino sources and other cosmic ray phenomena. This detector will provide us with a view of particle physics phenomena well above the window of energy soon to be opened by projects like the SSC, and as such represents a very important aspect of future research in high energy physics. The details of the detector layout are shown in a schematic view of the completed detector presented in Figure 1, and the details of the physics program and the capabilities of this detector have been presented in previous renewal requests to the DOE as well as in the original scientific proposal[1].

Over the course of the past year, this experiment has continued to make progress toward the final implementation of MACRO. During this time several milestones were reached, including the following:

- completion of the streamer tube system for the lower six modules.

- completion of the scintillation system for the lower six modules.

- began construction of the ATTICO expansion.

- decommissioned and recommissioned supermodule 1 with the other five modules to begin routine six supermodule data taking.

- began first major data taking run with six supermodules.

Over the past year several of our first physics papers have also begun to appear in particle physics and astrophysics journals. These papers have spanned the breadth of the MACRO physics program and illustrate the richness of this physics program.

In the sections that follow, we will discuss the progress made on physics analyses, detector construction and electronic development over the past twelve months, as well as present our plans for the coming funding cycle.

\section{A. MACRO Physics Results}

The MACRO collaboration has collected an impressive volume of data during 
the construction phase of the experiment. These data have been taken with only portions of the final MACRO system operating (eg. single or multiple supermodules, etc.). As a result, the first wave of refereed publications are now emerging from the group. These first publications ${ }^{(2,3,4,5,6,7,8]}$ have been based on theses that our students have presented in partial fulfillment of their Ph.D. requirements and/or conference presentations from the group. In addition to these physics results, we also have submitted an instrumentation paper ${ }^{[9]}$ which describes the setup of the first supermodule that we had been taking our early data on. A complete list of these publications appears in Appendix A where we list all the experimental group's publications for the iast year.

As for the future, MACRO is just now preparing to begin a long (6 month) data taking run utilizing the full lower six supermodules of the detector. This data will enabis us to extend significantly our searches for monopoles, WIMPS and other exotica and allow us to investigate in even more detail some of the interesting cosmic ray phenomena that we have observed to date. In addition to this new physics, there remain several papers which are currently in work within the collaboration which will be submitted for publication shortly.

MACRO is now entering its data taking phase in earnest and we expect the first results emerging now to set the pace for a steady flow of physics output over the next decade or more.

\section{B. MACRO Wave Form Digitizing (WFD)}

Our group has been involved in the MACRO collaboration R\&D effort to provide the necessary wave form digitizing system (WFD) for the MACRO detector's scintillator system over the last several years. In the past year MACRO has made significant progress toward the final implementation of the required WFD system. Approximately one year ago the MACRO WFD working group met to review the level of progress made by the three competing WFD systems and at this meeting it was decided to proceed toward the final MACRO implementation using the $\mathrm{BU}$ designed $200 \mathrm{MHz}$ system. The specifications for this system are outlined in the table below: 
1) $200 \mathrm{MHz}$ sampling. This sampling frequency is the minimum needed to digitize single photoelectron signals from the scintillation counters.

2) 8 bit linear (10 bit nonlinear) dynamic range. This dynamic range is necessary to view the wide range of monopole velocities we hope to be sensitive to with MACRO.

3) Effective time sampling window of $\sim 2$ msec. This allows us to study monopoles down to velocities of $\beta=10^{-5}$.

4) Zero suppressed data storage. In order to keep the amount of data transferred per event at a manageable level, the wave form information needs to be sparsified (32K bytes of sample storage).

5) An input sensitivity of 3-30 millivolts for a single photoelectron. This system should be able to identify single photoelectron pulses to give us the lowest possible velocity cutoff for monopoles (6 volts full scale).

6) Pre and post pedestal for baseline measurements. This feature will allow us to monitor the zero suppression activity for the real data.

7) Channel density of 4-16 channels/card depending on multiplexing option chosen.

8) Data Acquisition standard based on the VME bus ( $400 \mathrm{~mm}, 9 \mathrm{U}$ footprint).

At the WFD working group meeting, we followed this decision by laying out the plans for fully implementing the BU system for the detector. Our group went through each of the logical segments of the this system, reviewing carefully the performance and functionality of each stage of the system. Starting with the front end, we had a detailed discussion of the needs of MACRO, and how the non-linear front end might be implemented. The engineering group made a request to have several sample recorded waveforms from a pmt in order to have a better feeling for the types of signal information that they can expect and what then might be the ramification of changing the frequency response of the front-end, for example. We discussed the nonlinearity of the pmt and the range of input signals that we expect from the EMI tubes (0-5volt max). We agreed that the front end needs to map the 5 volt maximum amplitude phototube pulse into the 2 volt range of the FADC giving us an effective dynamic range from 0-1500 counts on the 8-bit ADC. 
One point that was raised concerning the design of the front end, was the need to be able to move the offset of the input to the FADC in order to be able to see baseline drifts and positive over shoot of pulses. The group felt that a sensible range of values for this offset adjustment was $1 \mathrm{mv}$ step from 0-256 mv. We also recommended that the threshold adjustment for the zero suppression also be modified to cover this same range of voltages $(0-256 \mathrm{mv}$ in $1 \mathrm{mv}$ steps).

Next our discussion turned to a system overview of sorts. We looked at the various control signals being used and how they might interact with the system in general and the ASIC in particular. At this point there were two very important issues raised regarding the design of device being presented to us. First in the prototype of the $200 \mathrm{MHz}$ system there had been a special treatment of the "terminal count" in the time circuit to identify roll-overs of the clock counter and enabling us to use the memory available to cover as long a time interval as possible. In the prototype, each time the clock reached full scale, a data recording cycle was initiated and an all-1's or all-0's word was written in to the time memory. In the latest version of the system this feature was lost. The second item that we discussed was the issue of recording the timing of the ]stop signal" being used to trigger the data. By recording accurately the time of this stop signal, we had planned to be able to make precise ( $\pm 5 \mathrm{~ns}$ ) timings between widely separated parts of the detector that might be triggered in an event. The current version of the WFD system has the timing information of these stop signal, but the granularity of the timing information was modulo $20 \mathrm{nsec}$.

Next we moved to the long delayed discussion of the ASIC itself. William Earle, lead engineer from Boston University, lead the discussion of this work, going through in detail the current design of this chip. There was general agreement that the ASIC looked to be appropriate for the job and apart from the two issues mentioned above.

Lastly we reviewed in detail the design of the ASIC which allows us to multiplex up to four channels to the input of the $200 \mathrm{MHz}$ FADC. This discussion resulted in several modifications being made to the ASIC design which necessitated another full round of design/simulation before we could send it off to be manufactured. We planned to make these changes and make one "final" review of the system by year's end before sending it off to the manufacturer. With this schedule for final design review, we expected to have the first sample ASICs available in early spring of ' 92 .

In parallel with ASIC work, we planned to have the BU group carry out the 
design of the printed circuit ( $\mathrm{pcb}$ ) for the WFD card and the TAMU group would be responsible for building the VME crate hardware for the system.

The ASICs arrived in April, 1992, however the work on the pcb was running a bit behind schedule due to some software problems with the CAD software packiage being used. The first prototype card was finally received in late April and a single group of four multiplexed channels was stuffed for testing.

The early testing of this system discovered several pcb design bugs that required patching, but the ASIC appeared to work without a hitch. After testing at BU on the bench, this prototype was taken to the Gran Sasso for further evaluation under final data taking conditions. However, due to lack of the requisite VME hardware at the Gran Sasso, these final tests could not be carried out at that time. Apart from this difficulty, the group felt that the ASIC design was sound and that we could start converging toward the final pre-production prototype pcb run.

We are currently revising the pcb design and expect to be finished with these changes by years end. We are then planning to make a first pre-production run to test a crate of WFDs before beginning full production. If we stay on our current schedule, we expect to be mounting the final WFD system on the detector in late spring/early summer of 1993.

\section{On/Off Site Technical Support of MACRO}

In addition tc our work with the WFD program, our group also has had the responsibility for many of the general electronics items for the MACRO scintillator system. The list of general electronics includes the phototube high voltage system, NIM and CAMAC crates and power supplies and general NIM and CAMAC hardware. Due to the remoteness of the experiment, our team has acted as the supply point and repair center for many of the items which require servicing or repair. Thus far it just has not been possible for MACRO to depend on repair facilities in Europe to service our needs in this area. Instead, we have determined through sometimes painful experience that it is often more expedient and cost effective to ship broken items back to the States for repair. During the course of the past year our group's technician, Ms. A. David, has serviced and repaired several shipments of broken equipment and returned it to the Gran Sasso. This service function is supported out of our group's base funding and represents a critical service for the MACRO team at this time. 
Along with the technical support that our group provides to the MACRO effort stateside, we have in the last year begun supporting a technician who is permanently stationed at the Gran Sasso. Ms. Ersilia Giusti has worked closely with the MACRO group for the past five years and previous to her joining our group was supported through the Indiana University group. Ms. Giusti is in charge of all shipping and receiving activity for MACRO U.S. groups and given the nature of the MACRO collaboration, this is a critical job for the success of our prograrn. In addition to the shipping/receiving duties, Ms. Giusti is also responsible for copying and distributing MACRO data tapes for the U.S. groups. Continued support of this MACRO effort from our base program is important for the continued success of the MACRO program.

\section{Plans for the Next Funding Cycle}

By the beginning of the 93-94 funding cycle, we anticipate being in full production of the WFD circuits. The TAMU team composed of Dr. R.C. Webb, new post doctoral research associate, Dr. Younan Lu, graduate student Ashutosh Sanzgiri and technicians A. David and E. Giusti will be responsible for testing of the WFD circuitry and will assist in the installation and commissioning of this

new system at the Gran Sasso. This group has been expanded considerably during the past twelve months and these new additions will enable TAMU to undertake a more active role in the MACRO program, both in the area of hardware implementation and software development.

In addition to our work on staging the WFD system and continuing to maintain various general electronics systems, we have begun to branch off into MACRO data analysis as well. In the past several months we have gotten the MACRO DREAM off-line package working on our group's microVAX. With a graduate student, A. Sanzgiri, and a post doc, Y. Lu, to lead this effort, for the coming cycle we are planning to get the MACRO package running on our new DECsystem, $5000 / 200$. This machine has the computing capacity to enable us to carry out a significant analysis program on any of the many MACRO physics topics.

We are looking forward with excitement to the commissioning of the WFD system on MACRO in the coming year. This new hardware will enable the collaboration to access all of the physics areas outlined in our original proposal. 


\section{References}

1. The MACRO Collaboration, "Proposal for a Large Area Detector Dedicated to Monopole Search, Astrophysics and Cosmic Ray Physics at the Gran Sasso Laboratory," November, 1984(unpublished).

2. S. P. Ahlen et al., "Arrival time Distributions of Very High Energy Cosmic Ray Muons in MACRO", Nucl. Phys. B, $\underline{370}$, 432(1992).

3. S. P. Ahlen et al., "Cosmic Ray Search for Strange Quark Matter with the MACRO Detector", Nucl. Phys. B., 24B, 191(1991).

4. S. P. Ahlen et al., "Study of Penetrating Cosmic Ray Muons and Search for Large Scale Anisotropies at the Gran Sasso Laboratory", Phys. Lett. B, $\underline{249}$, 149(1990).

5. S. P. Ahlen et al., "Measurement of the Decoherence Function with the MACRO Experiment at the Gran Sasso", Phys. Rev. D, submitted May, 1992.

6. S. P. Ahlen et al., "Search for Strange Quark Matter Using the MACRO Detector", Phys. Rev. Lett., submitted June, 1992.

7. S. P. Ahlen et al., "Study of Ultra-High Energy Primary Cosmic Ray Composition with the MACRO Detector", Pys. Rev. D, submitted March, 1992.

8. S. P. Ahlen et al., "Properties of MACRO in a First Search for Neutrino Bursts from Collapsing Stars", Astroparticle Physics, submitted May, 1992.

9. S. P. Ahlen et al., "First Supermodule of the MACRO Detector at the Gran Sasso", Nucl. Inst. and Meth., submitted May, 1992. 


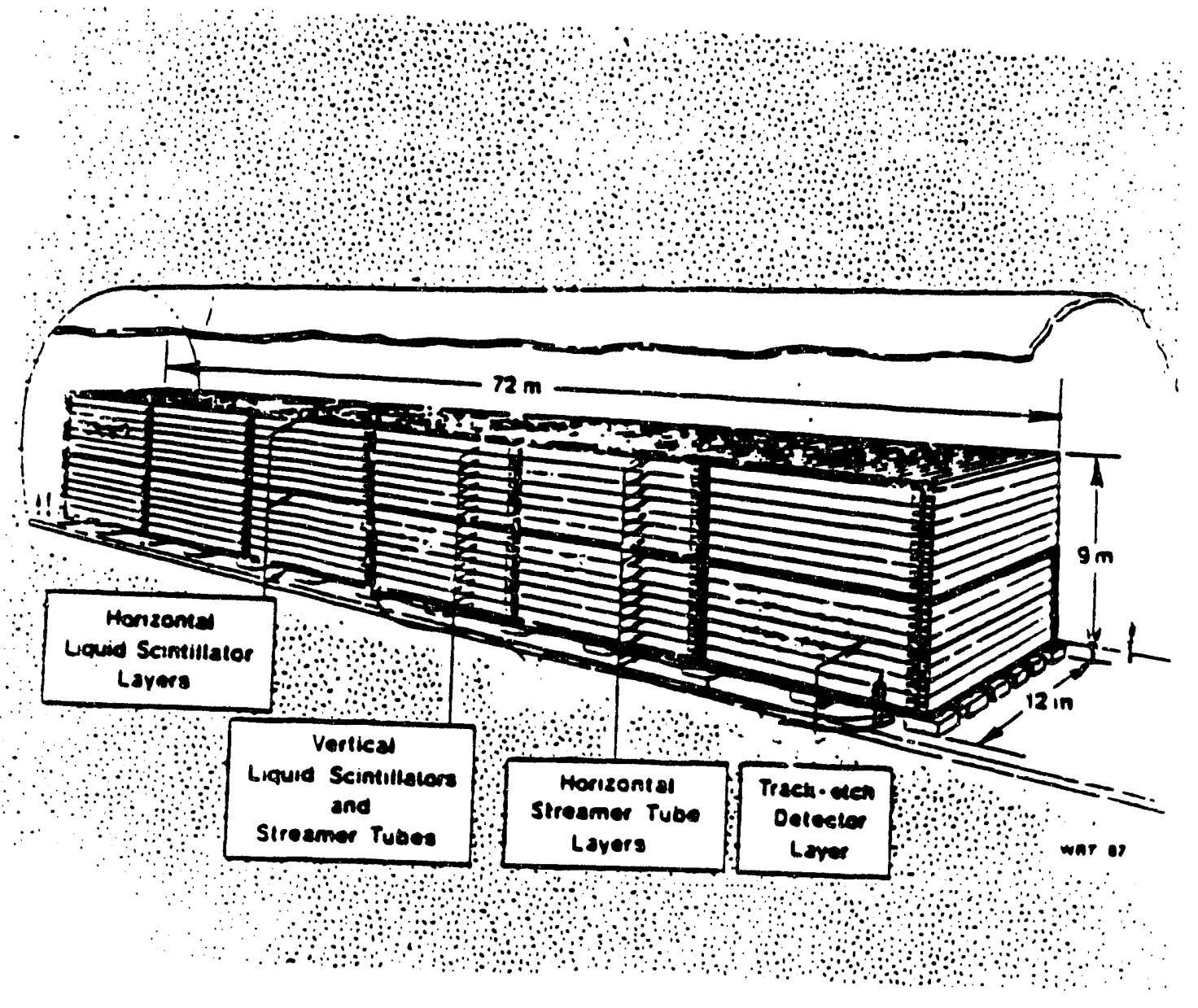

Figure 1. Layout of the MACRO detector. Shown are the six double decker supermodules. At this time the bottorn portions of these six modules have been fully constructed and supermodule one has been in operation for over a full year. Modules 2-6 are expected to be brought on-line in 1991 and the upper portion of the detector is scheduled for completion sometime late in 1992. 


\section{SSC Detector R\&D Program}

During the last funding cycle our program continued its $R \& D$ work on two major thrusts in SSC detector development. The first of these efforts has been the study of the use of liquid scintillator filled capillaries as the active medium in a radiation hard scintillating fiber calorimeter for SSC experiments and during the past twelve months we have been able to carry out beam tests on a prototype em calorimeter using this technique. The second area of investigation has been the study of gas microstrip tracking chambers for the precision tracking needs of SSC detectors and here, too, we have made significant progress aimed at using this technique in the CDF program before deploying it at the SSC. In the sections which follow, we will outline briefly the progress being made on each of these two $R \& D$ projects over the past year and discuss our plans for the coming funding cycle.

\section{A. Liquid Scintillator Based Fiber Calorimetry R\&D}

During the last decade a large number of detectors, employing liquid scintillators have been tested and used in various experiments around the world $\mathrm{d}^{[1,2,3,4,5]}$. Liquid scintillators are solutions with one or more scintillators in a solvent. The efficacy of these detectors with regard to various shapes and volumes has also been studied. As the energy of secondary particles emerging from high energy collisions has increased, the use of calorimetric techniques over those of standard magnetic spectrometry has becomes more prevalent. In addition, at the Superconducting Super Collider (SSC) we expect the particle flux in the forward region $(|\eta|>$ 3 ) to exceed that which could be dealt with using existing calorimeter technologies. Hence, we have been investigating the possibility of using a variant of the spaghetti calorimeter design for use in these rather inhospitable regions. In this design, we substitute scintillating liquid filled capillaries for the scintillating fibers of the spaghetti calorimeters. It is known that scintillating liquids have comparable response to ionizing radiation to that of their solid counterparts; while being much less susceptible to damage through intense radiation exposure.

In this study we have constructed a prototype electromagnetic calorimeter to study the performance of such liquid scintillator filled capillary calorimeters. In this section we will outline the investigations which we have carried out to study the basic operation of these liquid filled capillaries and present results obtained 
from the prototype calorimeter in response to a radioactive source, cosmic rays and test beam particles.

\section{Prirıciples of Fiber Calorimetry}

The total energy measurement of a high energy particle using a fiber calorimeter is accomplished by sampling the energy left behind in the scintillating material. The ionizing radiation detected in these fibers produces light through the excitation of fluors in the liquid scintillator medium, as the secondaries traverse the calorimeter. These photons are generated isotropically from the point of emission, but only those photons that undergo total internal reflection (TIR) and therefore get transmitted down the length of the tube containing the scintillator will be detected.

The angle at which TIR occurs between two media is called the critical angle, $\theta_{c}$, and is given by

$$
\theta_{c}=\sin ^{-1}\left(\frac{n_{m e d}}{n_{s c i n t}}\right)
$$

with $n_{\text {scint }}$ being the index of refraction of the scintillator and $n_{m e d}$ that of the surrounding medium, in this case the Teflon tube or $\mathrm{MgF}_{2}$ coating used in the study. This effect is illustrated in Figure 1.

For the Bicron scintillator oil, BC-517L, the index of refraction at the wavelength of maximum emission, $425 \mathrm{~nm}$, is $n_{\text {scint }}=1.47^{[6]}$. The index of refraction of Teflon is $n_{m e d}=1.376^{[7]}$, hence the critical angle, $\theta_{c}=69.5^{\circ}$, with reference to the normal to the two media. Therefore, with reference to the longitudinal axis of the Teflon tubes, all light travelling through the scintillator medium up to approximately $20^{\circ}$ from the Teflon tube surface will be totally internally reflected. This angle is approximately $17^{\circ}$ for $\mathrm{MgF}_{2}$ as its refractive index is $1.39-1.40^{[8]}$.

Another oil used in the study was the liquid scintillator used in the MACRO experiment ${ }^{[9]}$. This mixture is mineral oil based with a refractive index at the maximum wavelength of emission, $425 \mathrm{~nm}, 1.475$. With this oil, the critical angle therefore remains basically the same as that for BC517L.

The prototype calorimeter which we have constructed consists of a multilayered lead-scintillator system. The TIR medium between these layers provides a means of transfering the photons to the light detection system. The basic idea is to measure the light yield of the prototype calorimeter by sampling the number of charged shower particles generated in the lead sheets; liquid scintillator being the 
sigitial medium. Apart from light yield, another important factor characterizing the performance of the calorimeter is the loss of light (a) due to absorption by the scintillator medium and (b) due to imperfections in the TIR coating itself. These losses, which cause a reduction in the efficacy of the the TIR, can be characterized by an attenuation length, $\lambda_{\text {ef } f}$ defined as the total path length travelled by the photons after which the light intensity is reduced by a factor of $\frac{1}{e}$. Hence the intensity of light surviving to a distance $x$ from its creation point is given by

$$
L(x)=L_{0} \exp \left(\frac{-x}{\lambda_{e f f}}\right)
$$

where $\lambda_{e f f}$ is the effective attenuation length, $x$ is the path length travelled by the light and $L_{0}$ is the initial light intensity.

As a consequence of the two different mechanisms mentioned above, involved in the light loss process Eq.(2) can be rewritten as,

$$
L(x)=L_{0} \exp \left(-x\left(\frac{1}{\lambda_{\text {light }}}+\frac{1}{\lambda_{\text {scin }}}\right)\right)
$$

where $\lambda_{\text {light }}$ is the attenuation length of the light due to TIR process because of imperfections in the tube coating, $\lambda_{\text {scin }}$ is the attenuation length due to absorption by the scintillator material and $x$ is the path length travelled by the light.

Spectrophotometric measurements indicate that $\lambda_{s c i n}$ is about 2 meters for the Bicron scintillator oil and about 20 meters for the Macro scintillator oil. Therefore, if $\lambda_{\text {scin }} \gg \lambda_{\text {light }}$, Eq.(3) reduces to

$$
L(x) \approx L_{0} \exp \left(\frac{-x}{\lambda_{\text {light }}}\right)
$$

Hence we get,

$$
\lambda_{\text {eff }} \approx \lambda_{\text {light }}
$$

and is the attenuation length measured.

Two independent methods were used in measuring this effective attenuation length for these prototype calorimeter modules.

1) For individual modules (a) by using a radioactive source $\left(5 \mathrm{mCi},{ }^{137} \mathrm{Cs}\right)$, and (b) by using cosmic ray muons as the ionizing particles.

2) For a collection of seven modules in a close packed array (Fig. 2), using cosmic rays (muons) as the ionizing particles. 
The prototype electromagnetic calorimeter consists of seven hexagonal shaped modules with a detection length of $8.3 \mathrm{in}$. The modules are constructed from $1 \mathrm{~mm}$ thick sheets of lead, grooved along the length. Thirty four such sheets are glued to one another in layers with epoxy (Miller-Stephenson 828 epoxy/ V40 hardener) to form the absorber block. The grooves provide a housing for the Tefion tubes ( $1 \mathrm{~mm}$ inner diameter) which are spaced 0.1148 in. apart (Fig. 3(a)) along the surface of those sheets. The lead absorber block's outside surfaces are milled to form a hexagonal volume with its hexagonal face measuring $1.928 \mathrm{in}$. between two opposite apices (Fig. 3(b)).

In order to fill and retain the scintillator oil in the Teflon tubes, the ends of the module are capped with hexagonal acrylic disks that are strapped to the lead module with a stainless steel band. A 0.22 in. gap between the acrylic disk and the lead face forms a chamber to allow passage of the liquid scintillator the length of the module (Fig. 4(b)). On one end of the module, optical fibers are glued to the acrylic disk, such that each fiber individually faces a Teflon tube in the module and acts as a light guide (Fig. 4(a)). The other end of the module is used to circulate the liquid scintillator across the length of the module via two holes in the acrylic disk by means of an external siphoning system (Fig. 5). This ensures that all the Teflon tubes are filled with scintillator and are free of air bubbles. The other end of each optical fiber is glued to a 6 in. long solid hexagonal shaped ultraviolet transmitting (UVT) light collector that is in turn glued to a circular UVT acrylic disk measuring 2.125 in. in diameter (Fig. 6). A photomultiplier (PMT) placed against the face of this circular UVT disk forms the light detection mechanism for the module. A complete module assembly is shown in Figure 7.

The liquid scintillator in the Teflon tubes used in the study was the Bicron oil, BC-517L. It is a mixture of pseudocumene and mineral oil with the oil comprising over $75 \%$. The light from the primary fluor is emitted at $370 \mathrm{~nm}$, but due to wavelength shifters in the scintillating mixture, the maximum emission wavelength of the scintillator mixture is $425 \mathrm{~nm}$, compatible with the photocathode of the PMT used. Table $1^{[6]}$ gives some of the important physical constants of the liquid scintillator, BC-517L.

The photomultiplier tube used to measure the optical performance of the prototype detector was the EMI 9839B. This is a fast, linear focussed, end window 
P.IT approximately $51.0 \mathrm{~mm}$ (2.0 in.) in diameter and $150 \mathrm{~mm}$ (5.91 in.) in length. The overall electrical characteristics and rating of the PMT is given in Table 2. ${ }^{[10]}$

\section{Setup for Data Acquisition}

Three independent experimental methods were used to test the performance of the prototype detector. The first setup tested the performance of the nine modules (seven Teflon tube and two $\mathrm{MgF}_{2}$ coated modules) individually with a radioactive source $\left(5 \mathrm{mCi},{ }^{137} \mathrm{Cs}\right)$ (Fig. 8) and cosmic ray muons (Fig. 9). The second setup, which consistec of a collection of seven Teflon tube modules in a close packed array, was tested with cosmic rays (Fig. 10). The seven module close packed array was also used in a third experiment at Brookhaven National Laboratory (BNL) where it was subjected to an electron and muon test beam.

Single Module Measurements : In the single module setup a collimated radioactive source was kept at a fixed height above the module, and measurements of the total councs were taken along its $20.3 \mathrm{~cm}$ ( $8.0 \mathrm{in}$.) length at various points, for a fixed interval of time and recorded on a scaler. The experimental setup is shown in Figure 8 . The PMT voltages were set such that the single photoelectron signal was at 50-60 mV. Two independent measurements were made for each module, viz. the response of the module at $\geq \frac{1}{2}$ and $\geq 1 \frac{1}{2}$ photoelectron levels. This was accomplished by adjusting the threshold value of the discriminator to $30 \mathrm{mV}$ and $75 \mathrm{mV}$ respectively (Fig. 8).

Nine modules were tested by this method, seven of them with Teflon tubing as the TIR medium, and two with a coating of magnesium flouride $\left(\mathrm{MgF}_{2}\right)$ as the TIR medium.

Close Packed Array Measurements : The other setup used for testing the individual modules was in the cosmic ray telescope which was set up for this purpose. In this arrangement, signals from the module were gated with a coincidence pulse from two 'paddle' scintillation counters and a 'chip' counter, and the resulting pulse height spectrum was analyzed by a multichannel analyzer (MCA, LeCroy qVT 3001). The MCA was calibrated in terms of channe's per photoelectron. Figure 9 shows the experimental setup for each module.

In addition as mentioned in the description of the prototype detector earlier, 
the cosmic ray test of the close packed array consisting of seven Teflon tube modules was also carried out. The arrangement of the detectors is shown in Fig. $2 \&$ Fig. 10. Measurements were taken in this setup using a cosmic ray telescope with four overlapping plastic scintillator paddles, two on top and two on the bottom, sandwiching the prototype detector. A schematic of this setup is shown in Figure 10. In the setup illustrated, the output pulses of the PMTs from the seven modules are directly input into a 10-bit charge integrating analogue-to-digital converter (ADC, LeCroy 2249). The output pulses from the PMTs of the four paddle scintillators are split (in the ratio 4:1), with the larger pulse going via a discriminator into a 4 -fold coincidence and the smaller portion sent to the ADC for monitoring and for data acquisition. The 4-fold coincidence signal forms the gate for the ADC that triggers when a high energy cosmic ray muon passes a predetermined number of modules. The paddle data from the ADC was used to eliminate the triggered data that was due to noise. The width of the paddles was about one-third the length of the lead modules, so as to specify the region of detection, and therefore measure the performance of the whole detector as a function of distance from the light gathering system.

The close packed array of modules was also exposed to a high energy beam of pions and electrons during a SSCintCAL collaboration test beam run at Brookhaven National Laboratory (BNL) during the summer of 1992. Due to timing and space constraints during the test beam run this prototype system was exposed to only one energy of electrons $(10 \mathrm{GeV})$ With the close packed array oriented at $90^{\circ}$ to the incident beam direction. The results of these tests will be described in the next section.

\section{Data Analysis}

Single Module Measurements: The results of the experiments with the $\mathrm{Cs}^{137}$ source on the modules with a $1.0 \mathrm{~mm}$ inner diameter Teflon tube as the TIR medium are shown in Figure 11 and Figure 12 for the $\geq \frac{1}{2}$ and $\geq 1 \frac{1}{2}$ photoelectron measurements, respectively. The results with $\mathrm{MgF}_{2}$ as the TIR is given in Figure 13 and Figure 14 for the $\geq \frac{1}{2}$ and $\geq 1 \frac{1}{2}$ photoelectron measurements, respectively.

The average attenuation length of the Teflon tube modules is $12.79 \pm 1.33$ $\mathrm{cm}$. As deduced in the section on the principles of fiber calorimetry, this result is solely due to $\lambda_{\text {light }}$, the attenuation length of the light due to the tube coating, 
since $\lambda_{s c i n}$, the attenuation length of the Bicron scintillation oil, is just above 200 $\mathrm{cm}$. Tests with Macro scintillation oil which has a $\lambda_{\text {scin }}$ of about $1200 \mathrm{~cm}$ confirms this, since the effective attenuation length remains practically the same.

The results of the tests carried out with cosmic rays is given in Fig. 15 for Module $1 \& 2$, using Teflon TIR medium. The average attenuation length of the two Teflon modules was $12.07 \pm 1.11 \mathrm{~cm}$.

Close Packed Array Measurements: Two regions of the prototype detector were tested one near the light collecting face of the detector (close end) and the other, about $10 \mathrm{~cm}$ away from this face (far end). The characteristic spectra of the four padddle counters is shown in Figure 16 for the close end position and in Figure 17 for the far end position. Figure 18(a)-(g) shows the spectra of the individual modules for the close position and Figure 19(a)-(g) for the far position. These are characterized by a sharp initial peak, the pedestal, which is the result of a trigger when no charge is present at the anode of the PMT, and a broad peak at the mean number of photoelectrons times the gain of the photomultiplier. The position of the broad peak relative to the pedestal is a measure of the total number of photoelectrons.

In Figures 18 and 19 the first photoelectron peak can also be discerned. This facilitates the calibration of the prototype detector so that the total energy deposited can be calculated in terms of the number of photoelectrons. Figure 20 (a)\&(b) illustrates the sum-over-modules spectrum for the close and far ends, respectively (after normalizing with respect to the first photoelectron position in Module 1 and correcting for pedestal differences). The peak of the distribution occurs at 11.9 photoelectrons for the close end and 3.1 photoelectrons for the far end. Since the separation between the two measurements is $10 \mathrm{~cm}$, a value of 7.8 $\mathrm{cm}$ for the attenuation length is obtained.

For the geometry of the prototype detector, the average path length for a particle passing through it is $12.73 \mathrm{~cm}$. The effective density of the detector material (78\% lead, $22 \%$ oil) is $9.07 \mathrm{~g} / \mathrm{cm}^{3}$. The average depth therefore is $115.46 \mathrm{~g} / \mathrm{cm}^{2}$. Since cosmic ray muons, on average, deposit an energy of $\approx 1.5$ $\mathrm{MeV} / \frac{\mathrm{g}}{\mathrm{cm}^{2}}$, the energy depcsited in the detector is typically around $173 \mathrm{MeV}$. This corresponds to a light yield of $68 \pm 20$ photoelectrons $/ \mathrm{GeV}$ from the close end and $18 \pm 10$ photoelectrons/GeV from the far end.

Test Beam Measurements: As mentioned before the third experiment with the 
close packed array was carried out at BNL. In this experiment one data reading was taken with a single beam of $10 \mathrm{GeV}$ electrons perpendicular to the length of the array. The average pulse heights, measured in ADC counts, for three modules is shown in Figure 21(a),(b)\&(c) and the ADC sum for five modules normalized to 7 photoelectrons/ADC count Figure 22. The result at this energy showed that the average light yield was 1200 photoelectrons, (120 photoelectrons $/ \mathrm{GeV}$ ). The relative energy resolution $(\sigma / E)$ was $15 \%$.

\section{Conclusion}

The investigations carried out on the prototype detector, either as individual modules or as a close packed array have improved our understanding of the principles involved in building a liquid scintillator filled capillary calorimeter.

From the tests performed on the nine modules using the radioactive source as the ionizing particle, the average effective attenuation length for the seven scintillator filled Teflon tube modules in the $\geq \frac{1}{2}$ and $\geq 1 \frac{1}{2}$ photoelectron measurements was $14.44 \pm 1.19 \mathrm{~cm}$ and $11.14 \pm 0.60 \mathrm{~cm}$, respectively. The corresponding measurements for the two $\mathrm{MgF}_{2}$ coated modules was $5.18 \pm 0.04 \mathrm{~cm}$ and $3.82 \pm 0.03$ $\mathrm{cm}$. Table 3 shows the individual performance of each of these modules.

Tests with cosmic rays for two Teflon tube modules gave an average effective attenuation length of $12.07 \pm 1.11 \mathrm{~cm}$, consistent with our source measurements.

For the modules in a close packed array, tested with the cosmic telescope method the light yield was 11.9 photoelectrons for the close region and 3.1 photoelectrons for the far region of the detector. This corresponds to a light output of $68 \pm 20$ photoelectrons/ $\mathrm{GeV}$ for the close end and $18 \pm 10$ photoelectrons/ $\mathrm{GeV}$ for the far end. For the array at the BNL, with an electron beam, the result was 1200 photoelectrons @ $10 \mathrm{GeV}$, corresponding to $120 \pm 4$ photoelectrons/GeV, i.e. about twice th: yield obtained in the telescope method.

We can conclude from the various experiments carried out on the prototype detector that for the $1 \mathrm{~mm}$ Teflon coated tubes modules, and for the $\mathrm{MgF}_{2}$ coated modules, the outcome is mainly due to $\lambda_{\text {light }}$ (the attenuation due to imperfections in the tube coating). The poor optical performance of TEFLON coupled with its known radiation resistance make it an unlikely candidate for use in an SSC forward calorimeter. As for the $\mathrm{MgF}_{2}$ coating, although it has been observed to be radiation hard at those energies, the optical properties of the coating and substrate appear to be inappropriate for this application. 
To obtain longer attenuation lengths, it is evident that a new type of coating and a smoother surface to accommodate this coating is necessary. Preliminary studies with stainless steel tubes whose inner surfaces have been polished to a high degree of smoothness and coated with polydimethylsiloxane have yielded effective attenuation lengths in excess of $100 \mathrm{~cm}$, with Bicron oil, and $250 \mathrm{~cm}$ with MACRO oil. Although the particulars of the reproducibility of such tube performance has not been fully worked out and only a few such tubes have been tested, a prototype detector fabricated with these tubes should be capable of offering an excellent performance. This is the direction our present $R \& D$ program is heading, based on these results.

\section{B. High Precision Gas Microstrip Detectors for the SSC}

This is a research program for the production and testing of prototype highprecision gas microstrip particle track detectors. It is being carried out as a continuation of previous work supported by DOE and TNRLC and in conjunction with a large team of both US and foreign institutions to meet SDC milestones and goals. The devices also have a large number of potential applications in industrial $\mathrm{X}$-ray and medical imaging fields.

Our group has been involved in a study of combinations of substrate materials and configurations for the SDC intermediate tracking detector (ITD) system. We here summarize the research results in the past year and describe the research plan in the next cycle.

\section{Overview of SDC Intermediate Tracking}

The SDC has chosen gas microstrip detector (GMD) technology [11] for its tracking system in the intermediate angle region (from $10^{\circ}$ to roughly $30^{\circ}$ ).

The GMD devices replace the conventional pattern of anode and cathode wires in a proportional wire chamber with an etched pattern of parallel electrodes on the surface of a highly resistive (but slightly conductive) dielectric substrate. The gain of the GMD devices is reported to be $10^{3}-10^{4}$ for glass and plastic substrates. The anode/cathode pattern is produced by photolithographic techniques similar to those used by the semiconductor industry. The anode-to-anode strip pitch is usually about $200 \mathrm{hbox} \mu \mathrm{m}$. Typical anode and cathode strip widths are on the order of $10 \mathrm{hbox} \mu \mathrm{m}$ and $80 \mathrm{hbox} \mu \mathrm{m}$, respectively. The cathode strips are designed 
for field shaping around the anode and also for a short recovery time by providing a nearby return path for positive ions produced in the avalanche. The anode strip picking up a gas avalanche indicates the passage of a charged particle through the detector. We can achieve a position resolution of $58 \mathrm{hbox} \mu \mathrm{m}$ for $200 \mathrm{hbox} \mu \mathrm{m}$ pitch.

We can substantially reduce the occupancy expected in the SSC environment because of the small cell size provided by GMDs. Stable operation in the laboratory and in beam environments has been achieved. In the various designs produced up to now, however, some sensitivity to the choice of substrate, voltage settings and other operational parameters of these devices has been observed.

It should be noted that an international group has been formed to pursue the technical issues related to the use of GMDs within the SDC. Examples of such issues are the type of substrate and metallization pattern used, the comparison of chambers made with different production techniques and the development of an optimum method for electronic readout. Milestones for this work include a technical review of all tracking systems in January 1993, initiation of a prototype detector program (already under way) and production of a full-size detector tile in December 1993 (Figure 23).

\section{Substrate Research from Texas A\&M}

The choice of an appropriate substrate is the focus of an ongoing program of research within SDC by several collaborating institutions ${ }^{[1]}$. As a central activity, we have evaluated about 100 different glasses and plastics to find good substrate candidates $^{[12]}$. The first selection was made by measuring the ohmic behaviour and the surface resistivity $\left(\geq 10^{11} \Omega / \square\right)$ of the sample in air. The manufacturing processes (metalization, etching etc.) were also important factors for selection. Table 3 gives the parameters of nine chambers which were made by our group on different substrates and successfully tested.

For all nine of the chambers, we obtained good spectra from a $\mathrm{Fe}^{55} \mathrm{X}$-ray source. Figure 24 shows the spectra from GMDs built at Texas A\&M and Purdue Universities. Further results from measurements with these chambers are summarized in Ref. [12].

In the manufacturing processes, glasses are usually easier to handle than plastics. From the above list, the Fe/Vanadium glass and the ion-implanted Kapton look most promising. We also exposed the $\mathrm{Fe} /$ Vanadium chamber to a $7-\mathrm{GeV} / \mathrm{c}$ 
pion beam and obtained clean pulses from minimum ionization.

Of the test chambers cited above, only the ABS-copolyether has been tested with higher fluxes of $8 \mathrm{keV} \mathrm{X}$-ray from copper (up to $25 \mathrm{kHz} / \mathrm{mm}^{2}$ ), showing rate dependence. Similar tests for the other chambers still need to be done.

\section{Research Plan in the Coming Cycle}

Proposed Innovations in Production Methods : Previous detectors have all been manufactured by producing a metal pattern on the surface of a bulk conductive material. We would like to investigate an alternative production technique ${ }^{[13]}$ successfully used on a small scale by a research team at INFN-Pisa, which makes use the multi-layer process techniques available in the flat-panel and semiconductor industries. In this method, layers of controlled thickness and resistivity are grown using ion implantation and other techniques to yield a thin film of precisely known surface resistivity and dielectric strength on top of a buried layer of conducting material.

The thickness of the dielectric layer and the ion implantation will be varied in order to optimize the gain and stability of the resulting gas microstrip device. In addition, various options for segmentation of the metallization on the backplane layer will be explored, and other processing details will be investigated. An example of the mask pattern to be used in this effort is shown in Figures 25(a) and 25(b). The advantage of subdividing the backplane of a GMD device is that a second coordinate of readout complementary to that of the surface anode pattern can be obtained.

This technique has so far been applied only to small-scale devices on silicon wafers and quartz. We plan to develop this technique further by exploring various patterns of segmentation, by studying metallization and exposure techniques suitable for large-scale devices and by investigating the capacitance and surface resistivity properties of the resulting multi-layer substrate. The technology needed to produce multi-layer devices can be supplied in-house at Texas A\&M by combining new and previously existing equipment. These devices will be tested in comparison with ones produced by conventional techniques at other institutions associated with the program.

We are also pursuing production of the anode-cathode pattern, both in masks and for the actual GM detectors, by a new method available in industry known as excimer lithography[14]. This technique uses the output of excited dimer lasers 
in the deep UV region, $150-308 \mathrm{~nm}$, to expose metallized substrate and mask materials. The laser beam can either be used to ablate metal directly by direct writing with a small laser spot, or to expose or ablate a layer of photoresist or metal through a previously prepared optical mask. (See Figure 26) The method consists of photodecomposition followed by ejection of the photo-products as small molecules due to the increase in pressure. For organic materials such as photoresists, this phenomenon occurs above a threshold of $10-50 \mathrm{~mJ} / \mathrm{cm}^{2}$, depending on the material. Ablative photodecomposition of thin metals on a plastic substrate has also been observed. Ablation in one laser pulse of a thin metal layer can be achieved with laser fluences of $50-100 \mathrm{~mJ} / \mathrm{cm}^{2}$. This is one order of magnitude below the metal vaporization threshold, and the substrate remains cool.

In both techniques, high accuracy (on the order of $1 \mathrm{hbox} \mu \mathrm{m}$ to $2 \mathrm{hbox} \mu \mathrm{m}$ ) can be achieved over comparatively large $\left(18^{\prime \prime} \times 24 "\right)$ areas. This would be an attractive technique for producing gas microstrip devices that would be much larger than those that have been produced to date. Initial production of quartz/chrome masks has been made at Purdue University. We have begun working together with a laser lithography firm to test this technique on small scale devices. An example of the results of this effort is shown in Figure 27.

Tests of Detectors and Electronics: To lower costs and meet the schedule required by the SDC decision milestones, we chose early this year in the interests of speed to adapt an existing readout system ${ }^{[15]}$ for the CDF silicon microstrip vertex detector (SVX) project at Fermilab. This will enable us to proceed to the proposed tests without delay. The electronics for gas microstrips meet specifications similar to those used in the SVX, produced by Lawrence Berkeley Laboratory (LBL). The package consists of a pair of CAMAC modules for readout and control with a port card, receiver card and software for data acquisition and analysis. An example of the data path setup for the use of this system is shown in Figure 28. During this year, we will design the hybrid mount carriers for the SVX chips that are needed to match this system to the detectors described above, and will adapt them to other SDC gas microstrip modules. When SDC-specific preamplifier chips and the readout system ${ }^{[16]}$ are ready, we will convert to the use of this system. This work will be done at Texas Tech University and Fermilab.

We are preparing studies with a $\mathrm{Co}^{60}$ source at the Cyclotron Institute of Texas A\&M University. We will also set up high rate tests using an $\mathrm{X}$-ray machine at Trinity University to study rate dependence of gain and to investigate aging 
effects.

Relation to SDC Milestones: The SDC group has set a goal for completion of the above work as part of the ITD substrate search program by the middle of 1993. A selection of the microstrip tile design will be made at that time for construction of a full scale prototype. The prototype is scheduled to be completed by the end of 1994. The program of substrate development is underway and has resulted in several types of working devices.

Radiation tests, production of large patterned substrates and solutions for the many problems having to do with the production of large scale GMD devices are all important components of the SDC effort and will be needed to meet the group's milestones. To date, we have built only small-scale devices and instrumented a handful of lines for readout on each device. This has allowed us to determine that the basic design, substrate research, and implementation were sound. Now that rapid progress has been made on each of these fronts, we believe it is important to work towards building and instrumenting larger-scale devices. That is the focus of the program described here.

Overall Scope of Research: The evolution of the gas microstrip detector will be made by innovations in substrate materials, process methods and metallizations. In particular, we will pursue for the first time the use of excimer lithography to prodice large-scale GMDs, and ultra-thin dielectric devices, which provide the possibility of two-dimensional position readout. Parameters affecting performance, such as capacitance, dielectric thickness and surface resistivity and anode/cathode configuration will be explored. Electronics for large numbers of channels, needed to turn the devices into a practical detector, will be developed. Radiation tests and aging studies of previously made devices will continue.

The program described above will be carried out by four US Universities: Texas A\&M, Texas Tech, Trinity and Purdue. The scope of research is as follows:

(a) Metallization of plastic, glass, and multi-layer substrates with subsequent processing through laser and photo-lithography and etching to produce the needed anode/cathode pattern (with Purdue).

(b) Wire-bonding and connection of the devices to high voltage, electronics, and readout system for operational tests (Texas Tech).

(c) Radiation and high-rate testing of candidate substrates and chambers made 
from different materials (with Trinity).

(d) Comparison of performance among the various devices, including gain, surface resistivity, rate performance and resolution with several strips from multiple devices instrumented in a "telescope" fashion (with Texas Tech, Purdue and Trinity).

Our program is oriented toward the development of new technology as well as the completion of previous designs. This will increase the reliability of these designs for use in the SDC by increasing the number of options we have for dealing with potential problems that may arise. The present cost estimate for the ITD within SDC includes a large amount for contingencies to cover uncertainties in the performance of the detectors. We can reduce this cost by providing definite technical solutions to issues relevant to the ITD design.

Collaboration with other SDC institutions is essential to the program. Thus, the work is also being pursued with a group that includes significant foreign collaborators.

In addition, the research will provide advancement in manufacturing techniques, improvement of our ability to characterize and make basic measurements upon these devices. The technique and device can also be used in the X-ray imaging and medical diagnostic fields.

Finally, we believe gas microstrip detectors to be of basic interest to the high energy physics community. The research described here will substantially increase our knowledge of how to build these detectors and thus help pave the way for their general use as a new class of low mass, high rate particle detectors. 


\section{References}

1. V.G. Zinov et al, Instr. Exp. Tech. 25 (1982) 303.

2. V.V. Filchenkov et al, Nucl. Instr. and Meth. A245 (1986) 490.

3. S. Mellema and J.S. Petler, Nucl. Instr. and Meth. A242 (1986) 265.

4. M. Bonesini et al, Instr. Exp. Tech. A261 (1987) 471.

5. The MACRO Experiment at Gran Sasso, Italy.

6. Bicron Corporation, 12345 Kinsman Road, Newbury, Ohio 44065.

7. J. Brandrup and E.H. Immergut, Polymer Handbook, V-29. Wiley Interscience, (1975).

8. Encyclopedia of Physics.

9. J.P. Petrakis et al, Nucl. Instr. and Meth. A268 (1988) 256.

10. EMI Photomultiplier Catalog 1979, THORN EMI Gencom Inc.

11. SDC ITD Group (B. Foster et al.), "Intermediate Angle Track Detector Conceptual Design Report," SDC-92-00171, UR-1246 (Jan. 1992); SDC Collaboration, "Technical Design Report," SDC-92-201 (April 1992).

12. E.F.Barasch and T.B.Kasprowicz, "Substrate Resistivity Measurements," SDC-92-314 (1 September 1992); E.F.Barasch et al., "Gas Microstrip Detectors on Polymer, Silicon and Glass Substrates," SDC-92-318 (8 September 1992), Proceedings of the 3rd International Conference for Advanced Technology and Particle Physics, Como, Italy, 22-26 June 1992.

13. See for example F. Angelini et al., INFN PI/AE 92/01 (14 April 1992), Proceedings of the 6th International Wire Chamber Conference, Vienna, 17-21 February 1992; R. Bouclier et al., Proceedings of the 6th International Wire chamber Conference, Vienna, 17-21 February 1992.

14. R. Srinivasan, "Kinetics of the Ablative Photodecomposition of Organic Polymers in the Far UV," J. Vac. Sci. Tech. B 1, 923 (1983); Lankard and Wobold (IBM), "Excimer Laser Ablation of Polyimide in a Manufacturing Facility," Appl. Phys. A 54, 355 (1992); U. Sowada et al., "Excimer Laser 
Processing of thin Metallic Films on Dielectric Substrates," SPIE Vol. 801 High Power Lasers (1987). See also "Excimer Laser Lithography," by K. Jain. SPIE Optical Engineering Press (1990).

15. C. Haber et al., "Design and Prototype of the Frontend Readout System for the CDF Silicon Vertex Detector," IEEE Trans. Nucl. Sci. 37, p1120-1126 (1990); S.A. Kleinfelder et al, "A Flexible 128 Channel Silicon Strip Detector Instrumentation Integrated Circuit with Sparse Data Readout," IEEE Trans. Nucl. Sci. 35, p171\%175 (1988).

16. J. DeWitt, A. Grillo, B. Hubbard, D. Hutchison, "Digital Readout Sequencer (DRS) Module Performance Specification," UC Santa Cruz note in preparation. 
Table 1. Physical constants of liquid scintillator BC-517L

\begin{tabular}{|c|c|}
\hline Light Output \% Anthracene & $30 \%$ in Air \\
\hline Decay Time, $\mathrm{ns}$ & 2.0 \\
\hline Density, $\mathrm{gm} / \mathrm{cm}^{3}$ & 0.86 \\
\hline Index of Refraction at $425 \mathrm{~nm}$ & 1.47 \\
\hline Wavelength of Max. Emission, $\mathrm{nm}$ & 425 \\
\hline Flash Point, ${ }^{\circ} \mathrm{F}$ & $215\left(101.8^{\circ} \mathrm{C}\right)$ \\
\hline No. of C Atoms per $\mathrm{cm}^{3}$ & $3.68 \times 10^{22}$ \\
\hline No. of h Atoms per $\mathrm{cm}^{3}$ & $7.40 \times 10^{22}$ \\
\hline Ratio H:C Atoms & 2.01 \\
\hline No. of Electrons $\mathrm{cm}^{3}$ & $2.95 \times 10^{23}$ \\
\hline
\end{tabular}

Table 2. Electrical characteristics of photomultiplier EMI 9839B

\begin{tabular}{|c|c|}
\hline Spectral Response \& Window & Bialkali/B \\
\hline No., Type \& Material of Dynodes & 12, Linear Focused, BeCu \\
\hline Effective Cathode Dia., (mm) & 45.0 \\
\hline Quantum Efticiency (\%) at Peak & 27 \\
\hline Anode Sensitivity, A/lm at $V_{k-a}$ & 500 at 1900 \\
\hline Gain & $6.7 \times 10^{6}$ \\
\hline Dark Emission, $I_{a}$ (dark) nA & 2.0 \\
\hline Rise Time, ns & 3.0 \\
\hline
\end{tabular}


Table 3. Individual module performance using different ionizing particles .

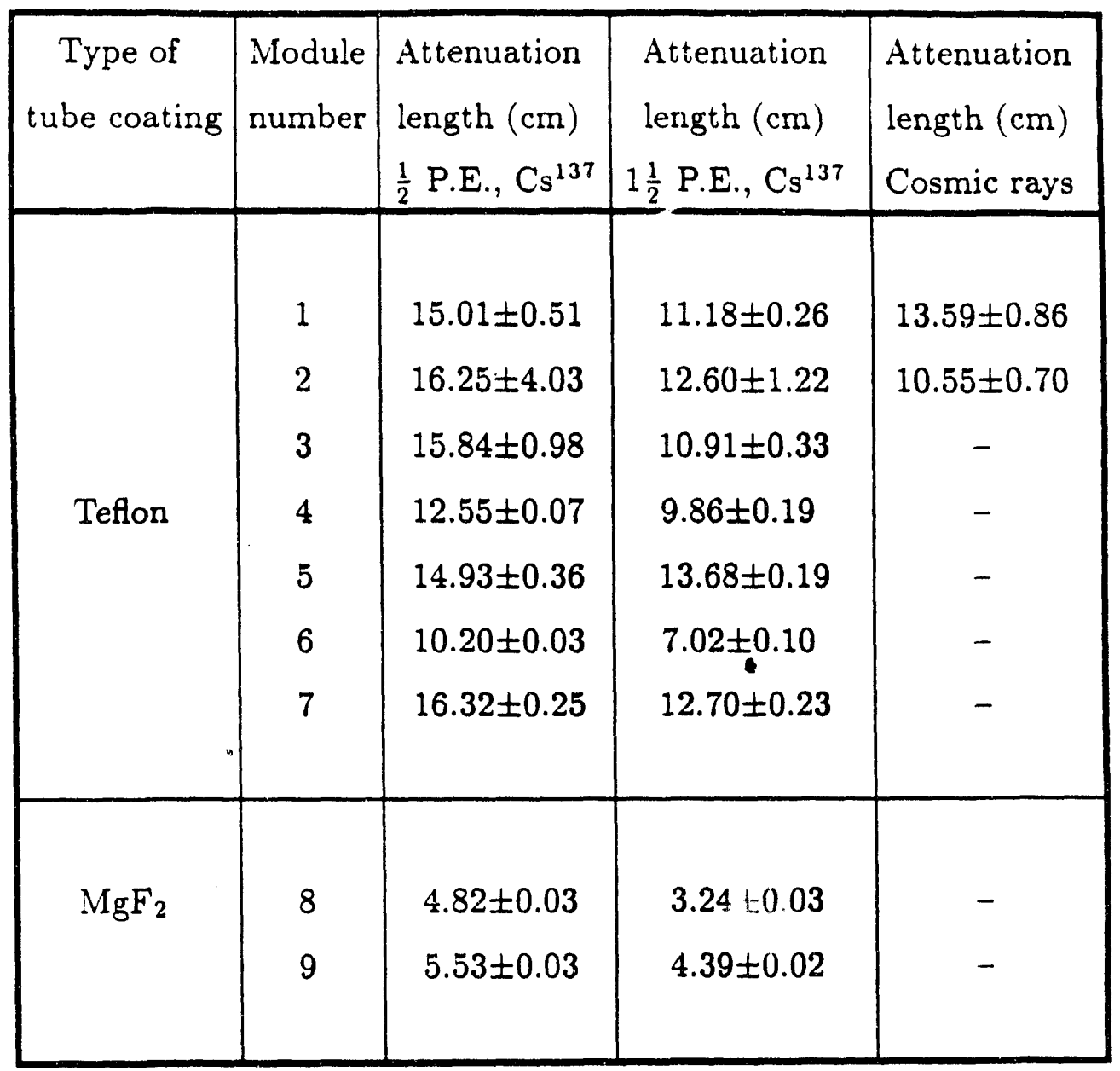


Table 4: Substrate Parameters for

Several Gas Microstrip Chambers

\begin{tabular}{|c|c|c|c|}
\hline Substrate & $\begin{array}{c}R_{\text {surface }} \\
{[\Omega /]}\end{array}$ & $\begin{array}{l}\text { Thickness } \\
{[\mathrm{mm}]}\end{array}$ & $\begin{array}{l}\text { Pitch } \\
{[\mu m]}\end{array}$ \\
\hline ABS-copolyether & $2 \times 10^{11}$ & 1.55 & 300 \\
\hline Upiley "S" Polyimide & $4 \times 10^{16}$ & 0.127 & 100,200 \\
\hline Tedlar & $1 \times 10^{16}$ & 0.10 & 200 \\
\hline Texin 4215 & $2 \times 10^{13}$ & 0.25 & 200 \\
\hline Ion-implanted Kapton & $4 \times 10^{13}$ & 0.127 & 100,200 \\
\hline Non-planar silicon & $>10^{17}$ & 0.275 & 200 \\
\hline Ceramic & $7 \times 10^{14}$ & 0.25 & $20 \mathrm{~J}$ \\
\hline Tempax borosilicate glass & $3 \times 10^{15}$ & 1.75 & 300 \\
\hline $\mathrm{Fe} /$ Vanadium glass & $2 \times 10^{12}$ & 1.0 & $\angle 00$ \\
\hline
\end{tabular}




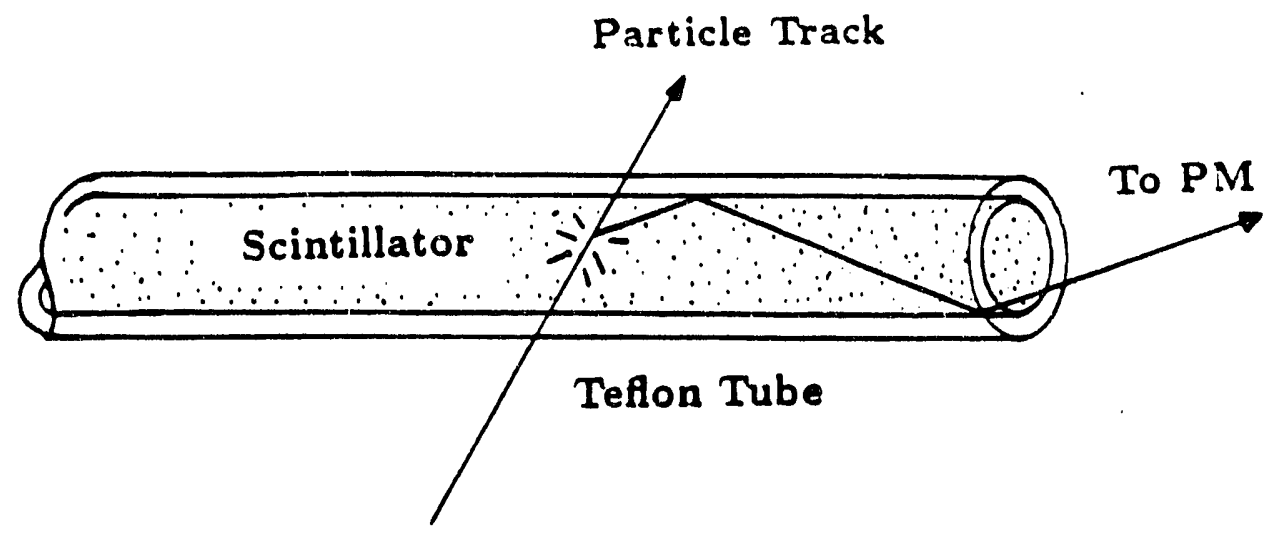

Figure 1. Diagram of a liquid scintillator filled Teflon tube.

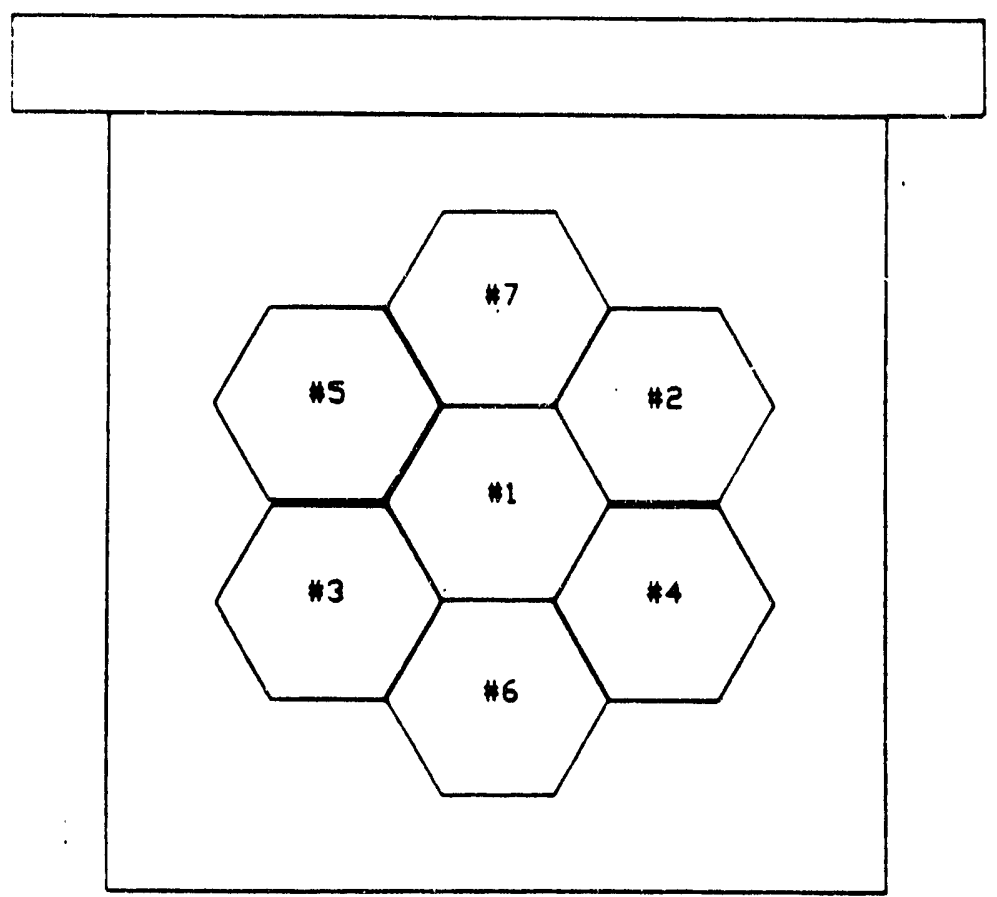

Figure 2. Cross section of lead modules in contiguous position. 


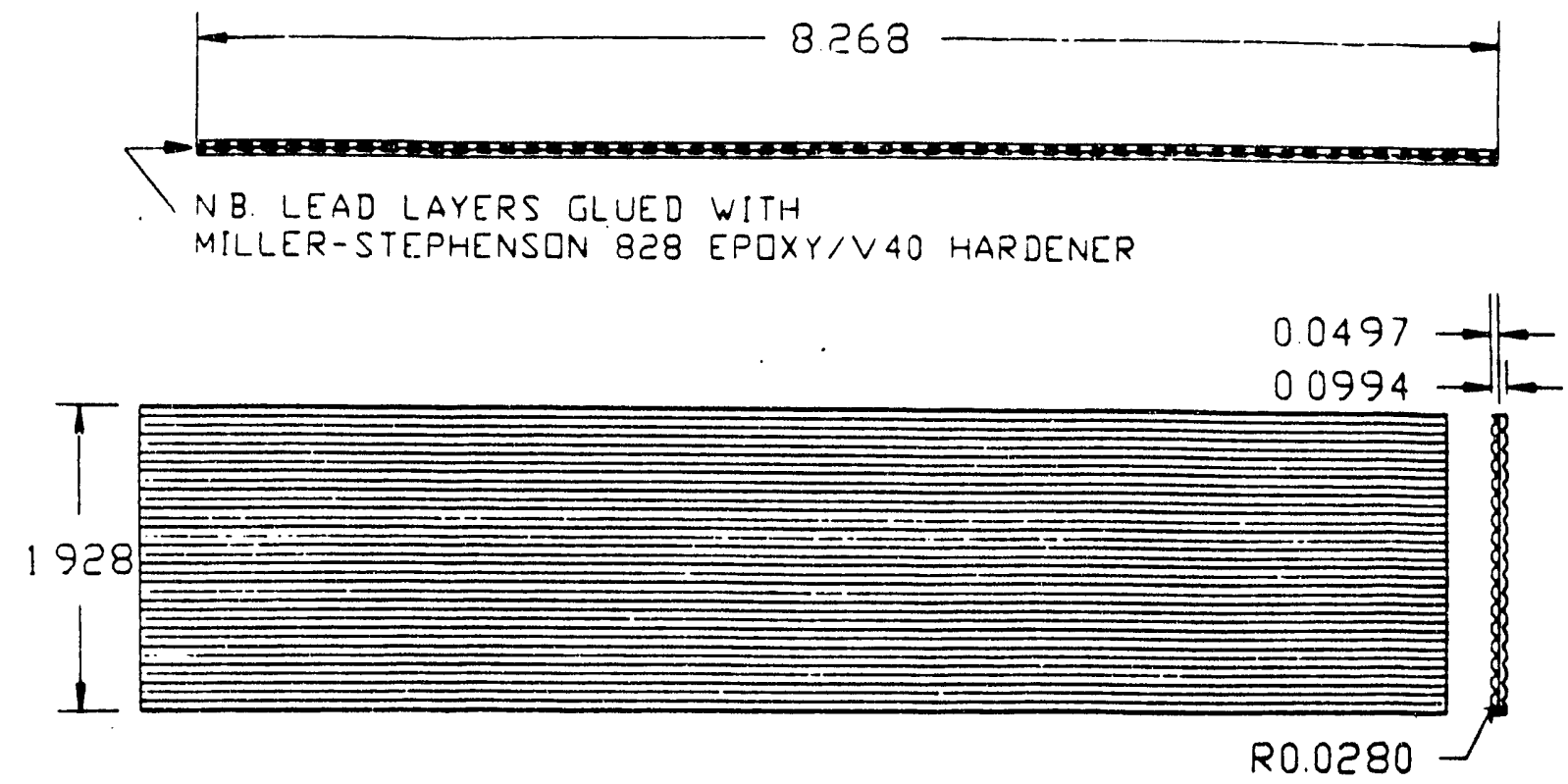

(a)

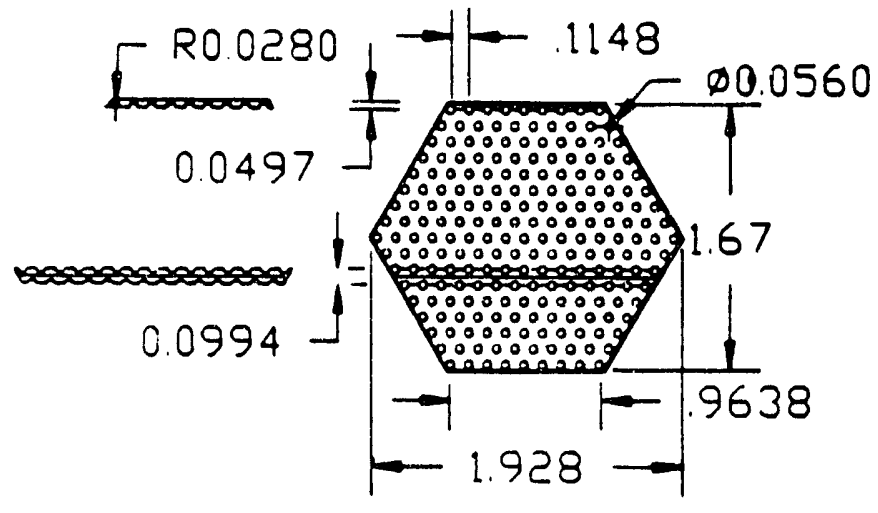

(b)

Figure 3. (a) Longitudinal \& (b) End view of modules made from grooved lead plates (all dimensions in inches). 


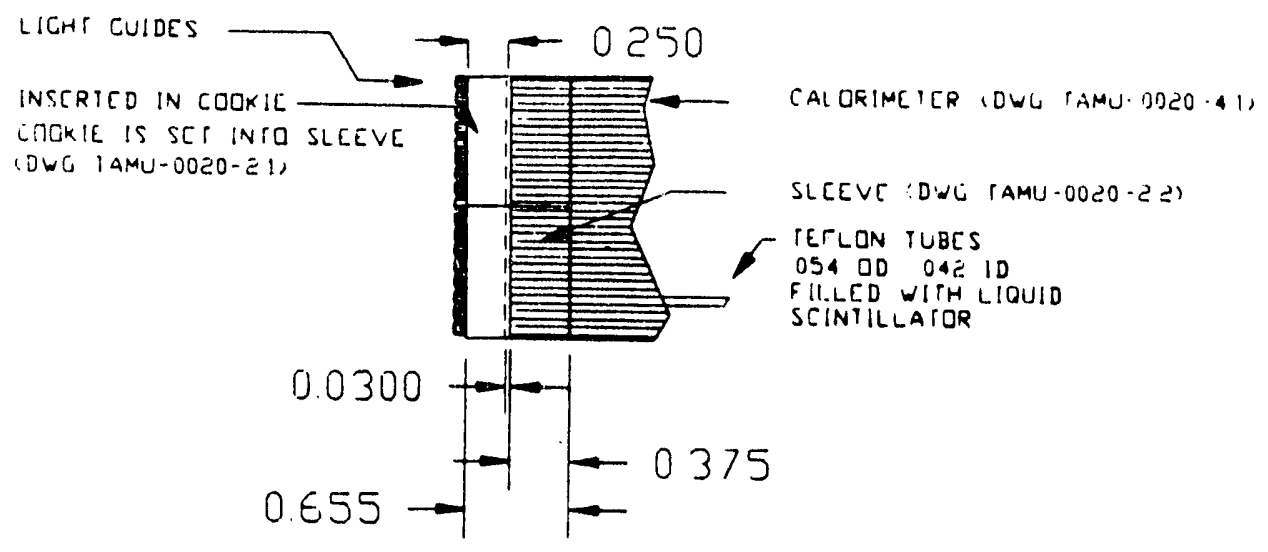

(a)

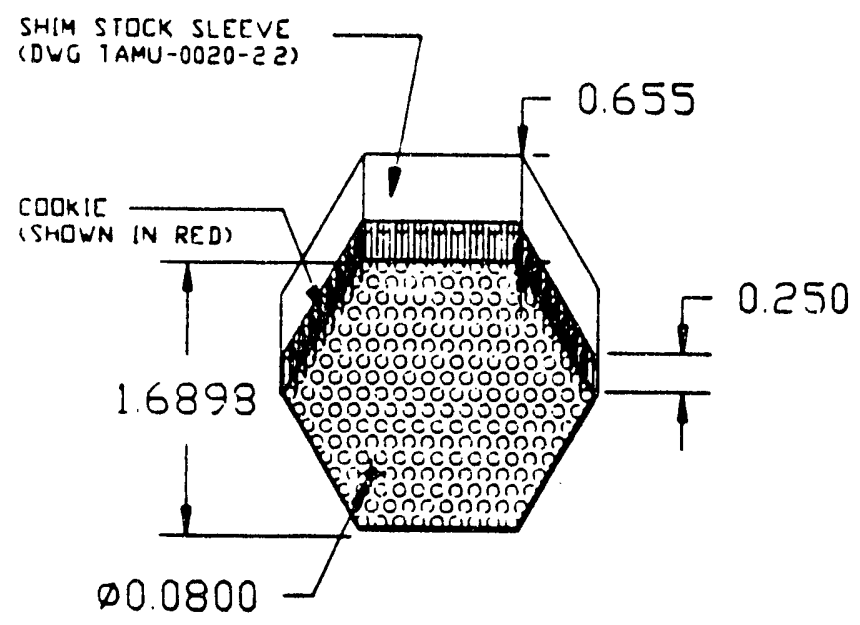

(b)

Figure 4. (a) Side \& (b) End view of hexagonal shaped acrylic disk and sleeve assembly (all dimensions in inches). 


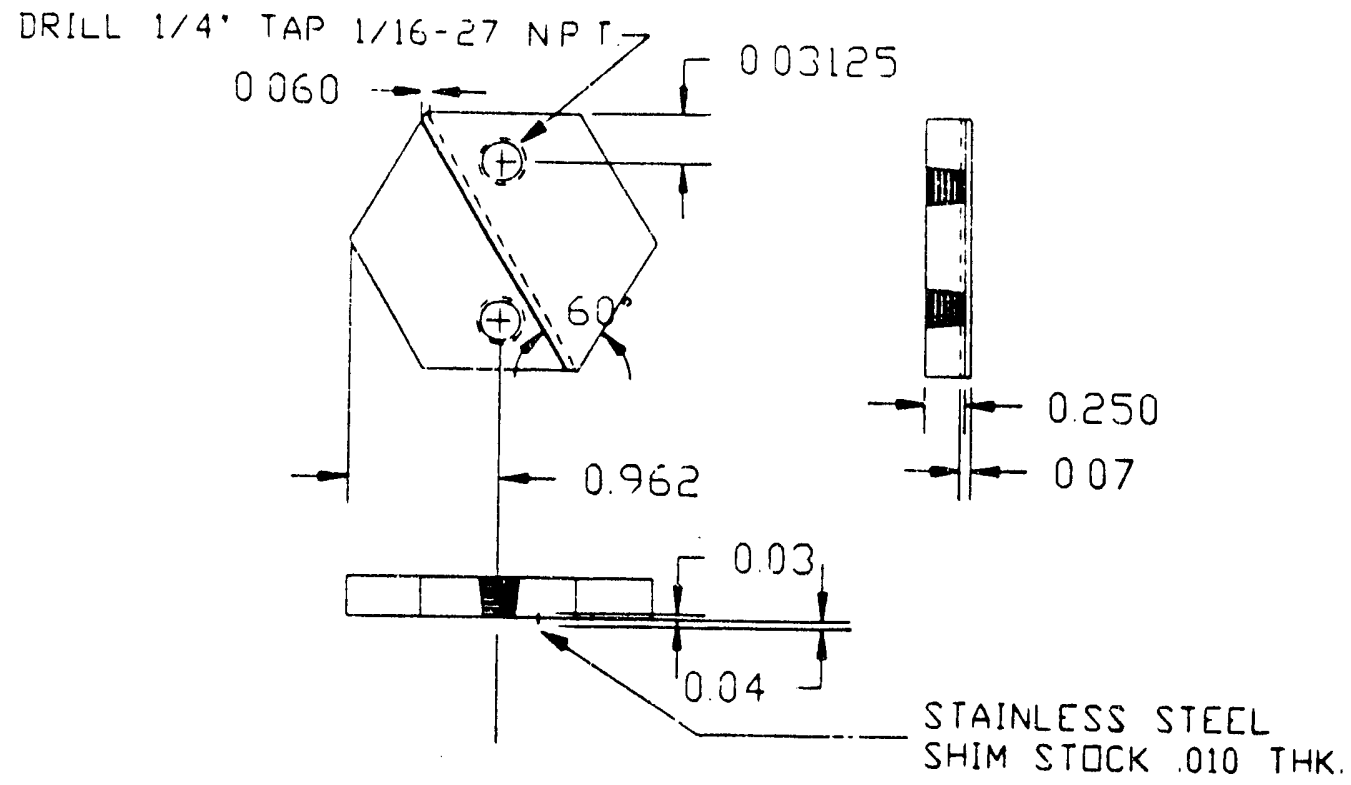

Figure 5. Hexagonal acrylic disk with partition between two tapped holes to siphon liquid scintillator through tubes (all dimensions in inches.)

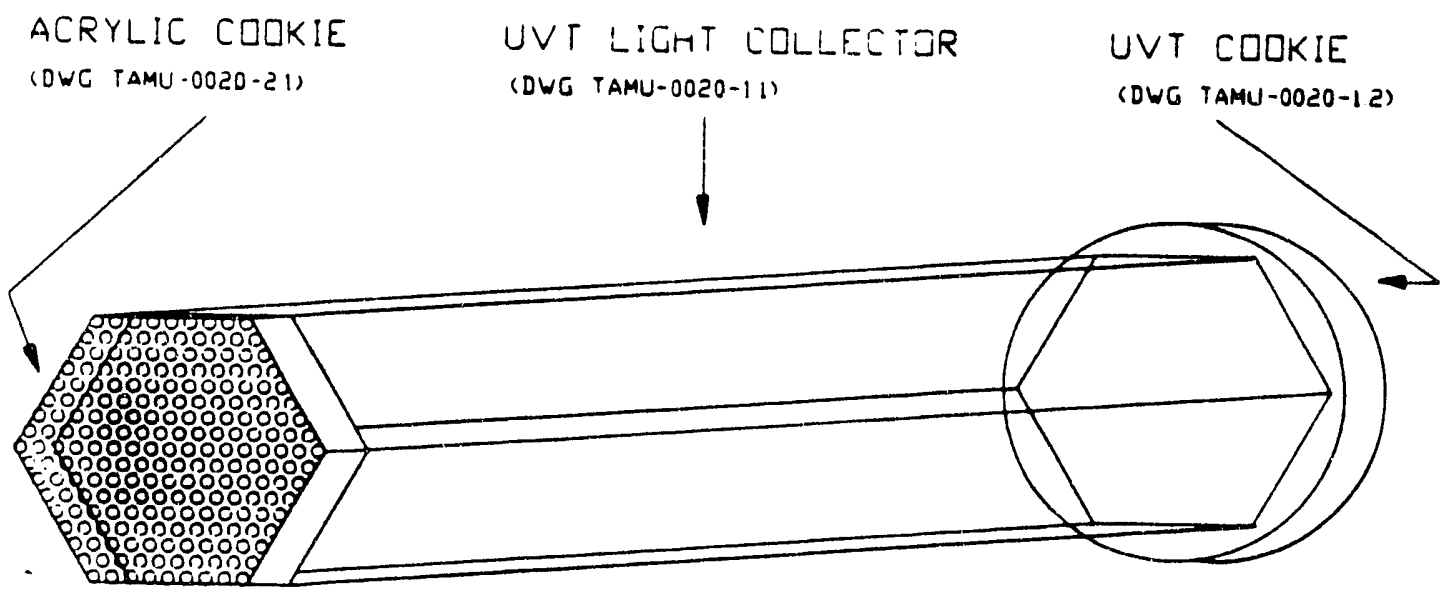

Figure 6. Assembly of light guide acrylic disk with UVT light collector and UVT transmitting circular disk. 


\section{To PMT}

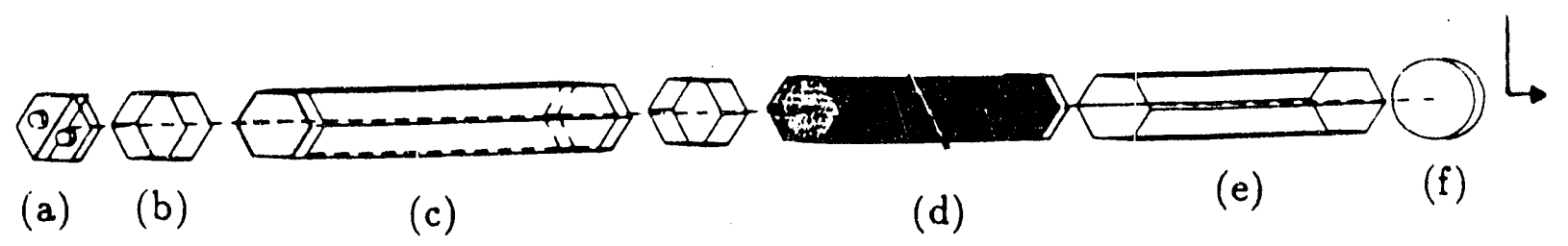

(a) (b)

(c)

(d)

(e)

(f)
(a) Hexagonal acrylic disk with tapped holes.
(b) Stainless steel shim stock sleeve.
(c) Hexagonal lead module.
(d) Light guides.
(e) Hexagonal UVT transmitting light collector.
(f) Circular UVT transmitting acrylic disk.

Figure 7. Drawing a typical assembled module. 


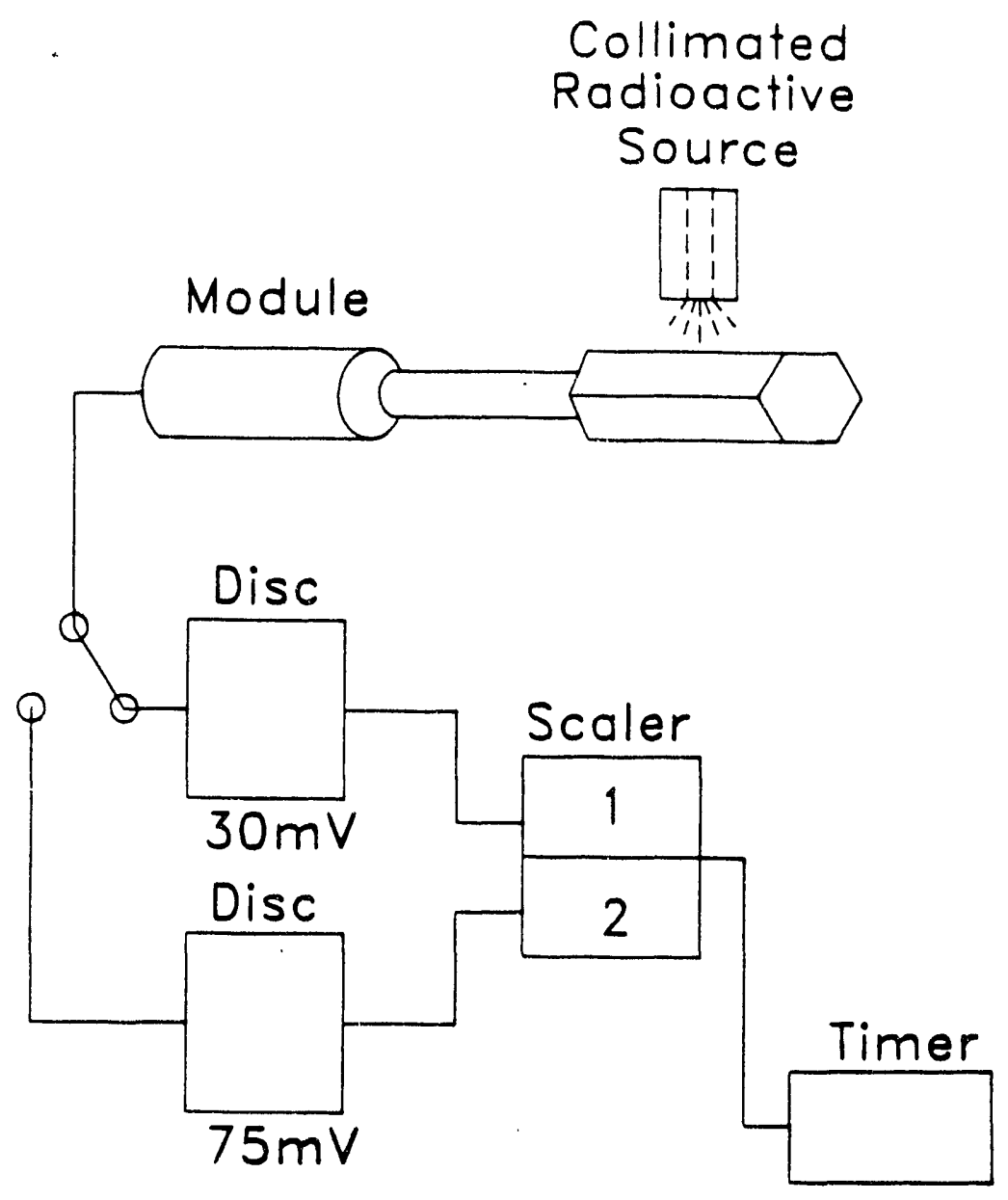

Figure 8. Setup for single module experiment with radioactive source. 


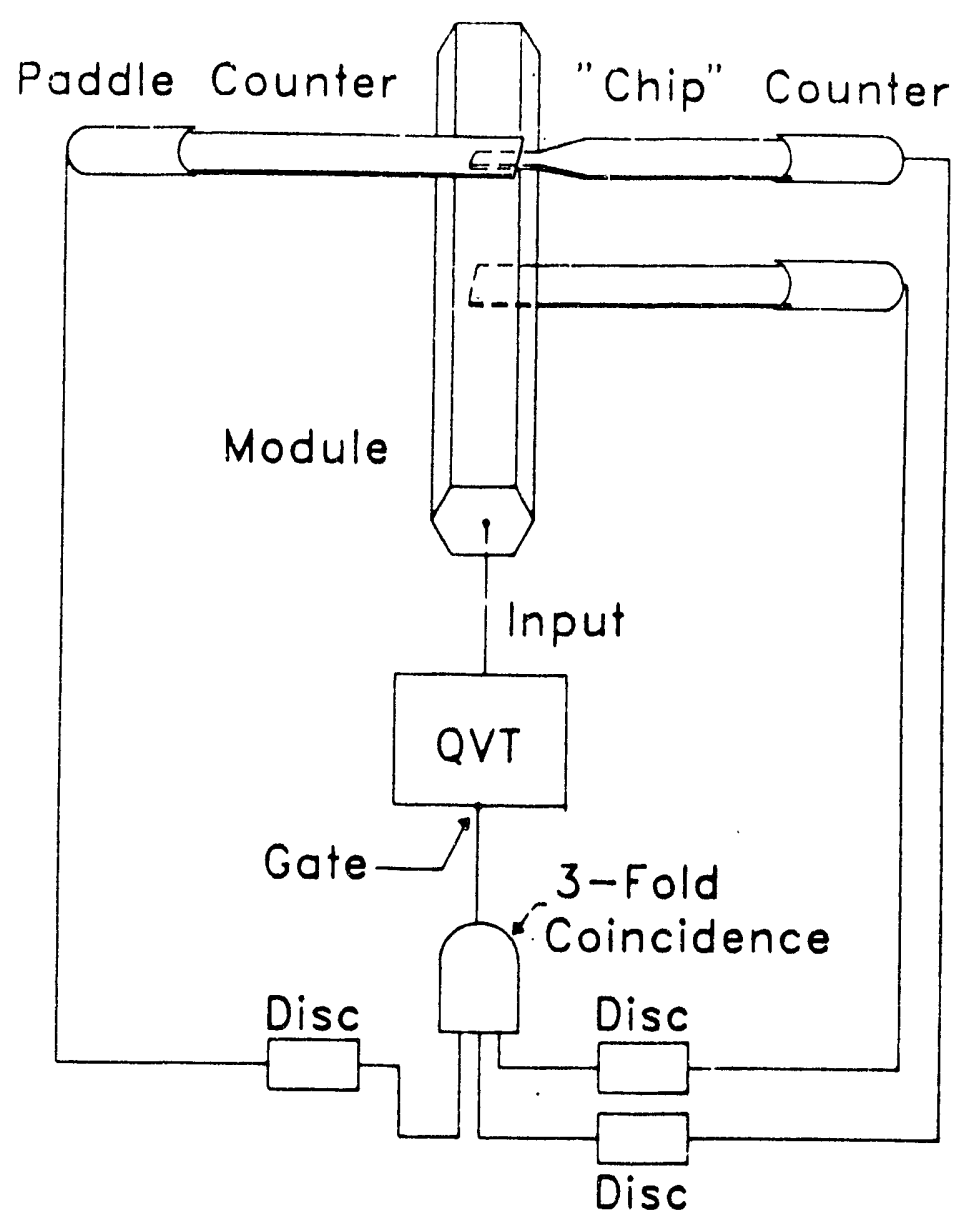

Figure 9. Setup for single module experiment with cosmic rays. 


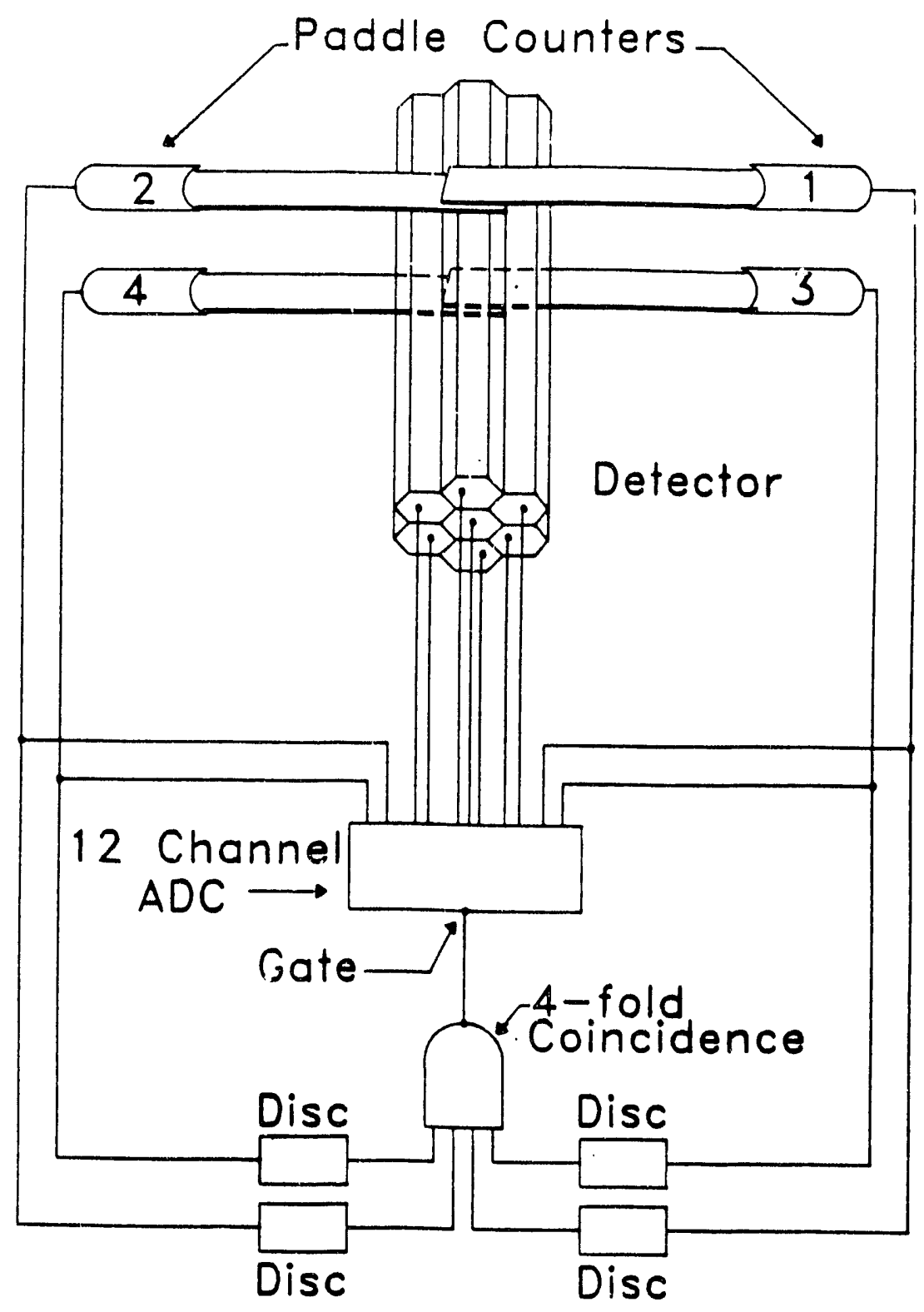

Figure 10. Experimental setup for contiguous module position measurements with cosmic rays. 


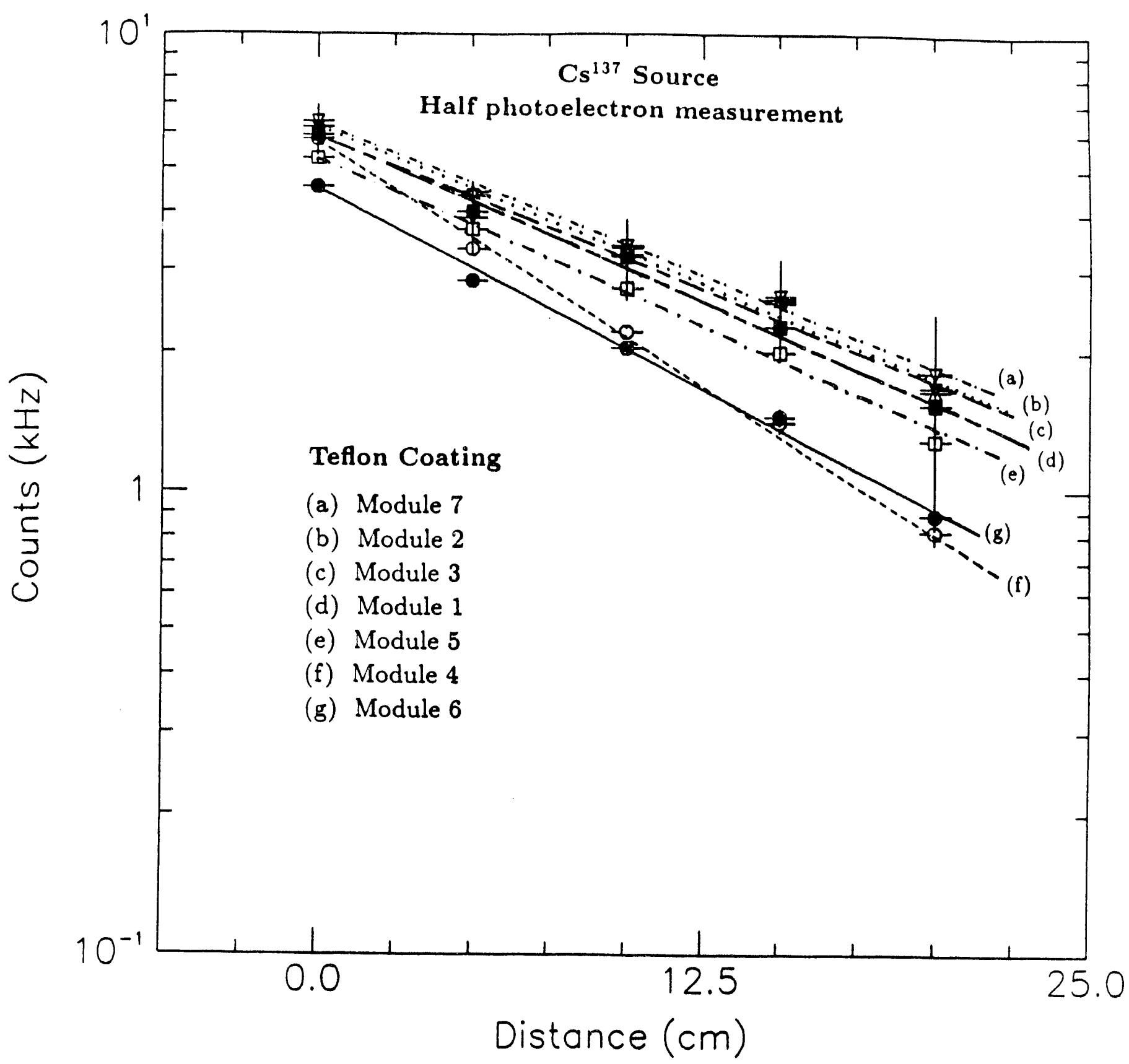

Figure 11. Half photoelectron measurement for individual Teflon tube modules using the radioactive source, $\mathrm{Cs}^{137}$. 


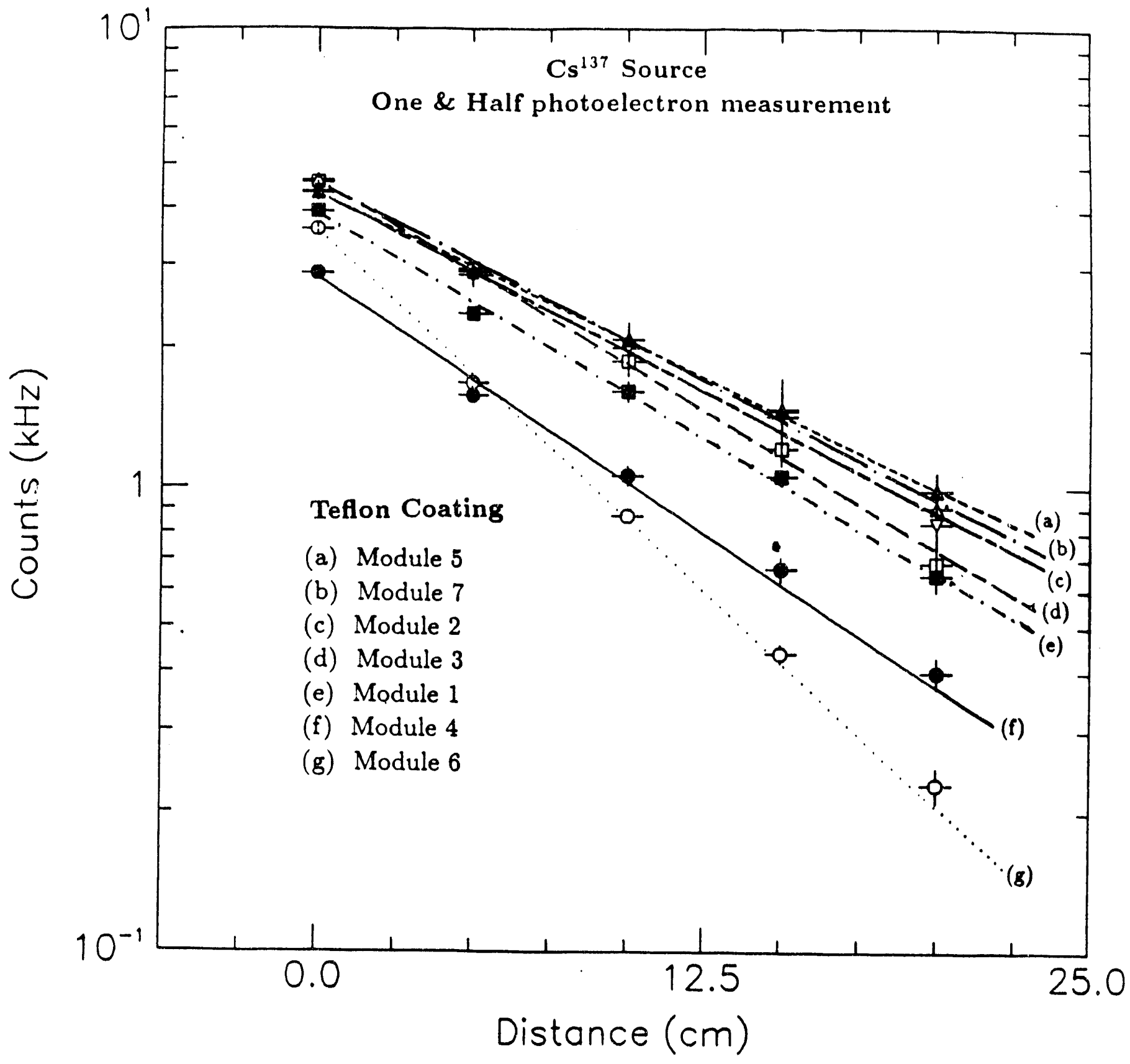

Figure 12. One \& a half photoelectron measurement for individual Teflon tube modules using the radioactive source, $\mathrm{Cs}^{137}$. 


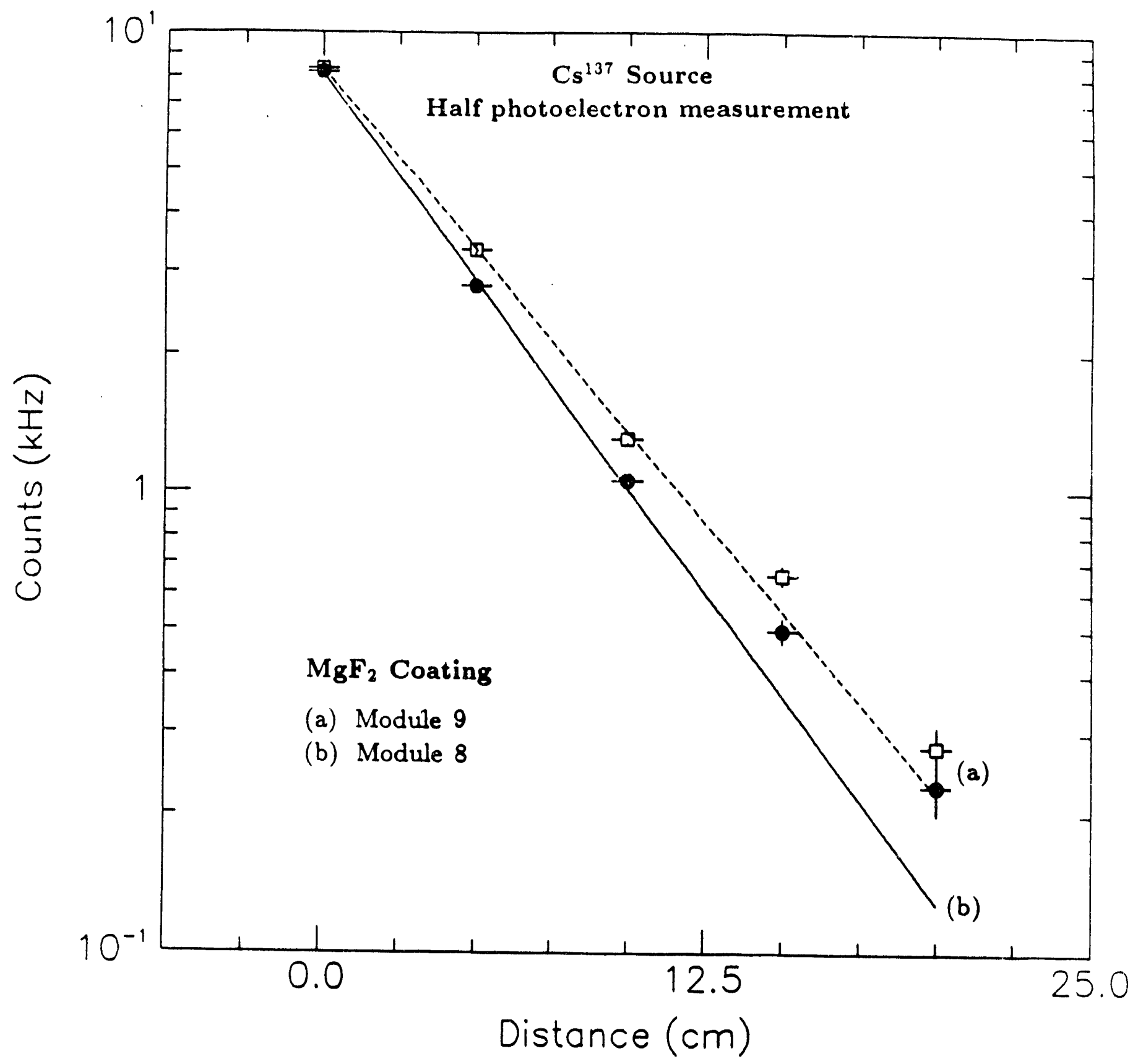

Figure 13. Half photoelectron measurement for individual $\mathrm{MgF}_{2}$ coated modules using the radioactive source, $\mathrm{Cs}^{137}$. 


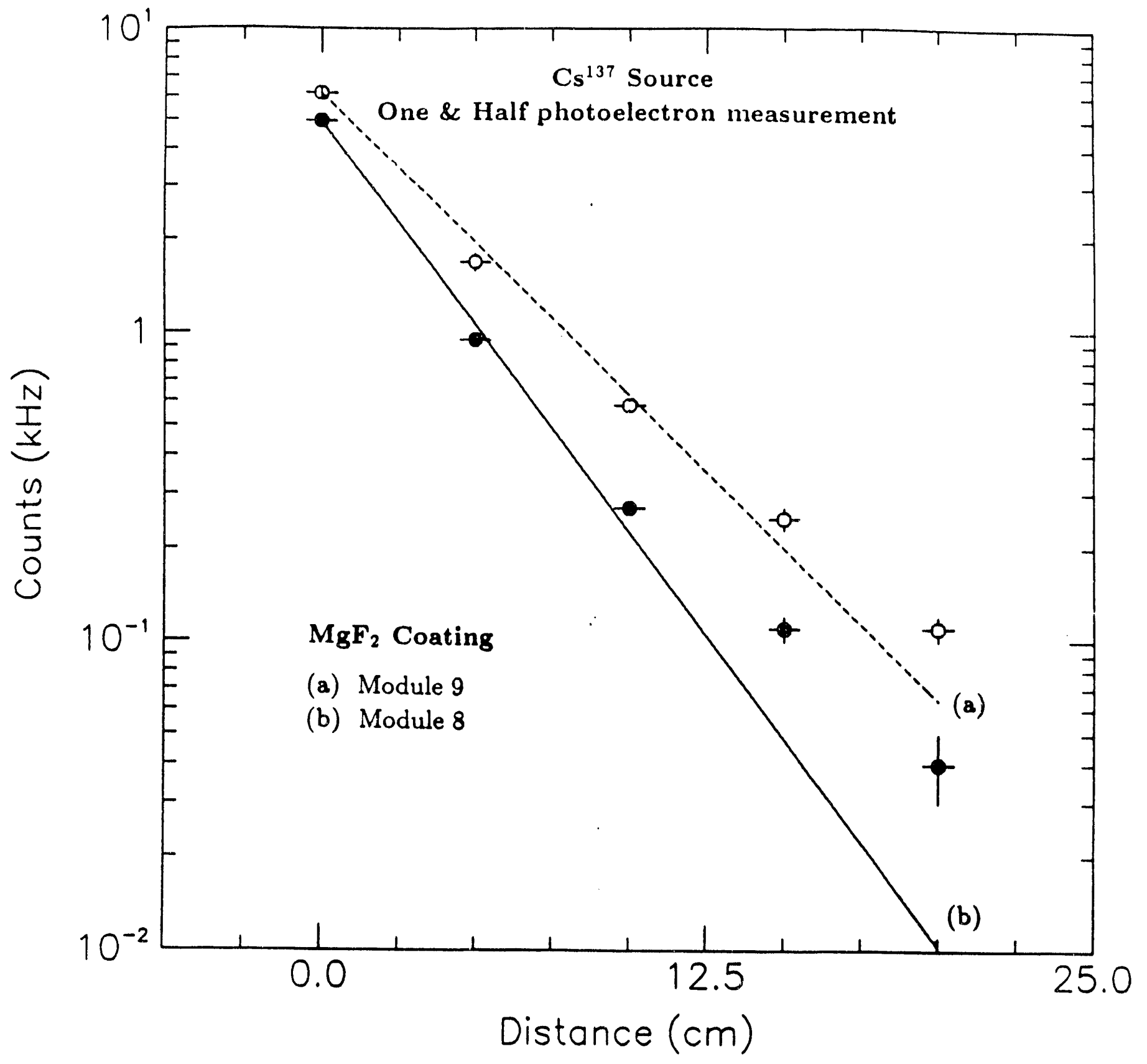

Figure 14. One \& a half photoelectron measurement for individual $\mathrm{MgF}_{2}$ coated modules using the radioactive source, $\mathrm{Cs}^{137}$. 


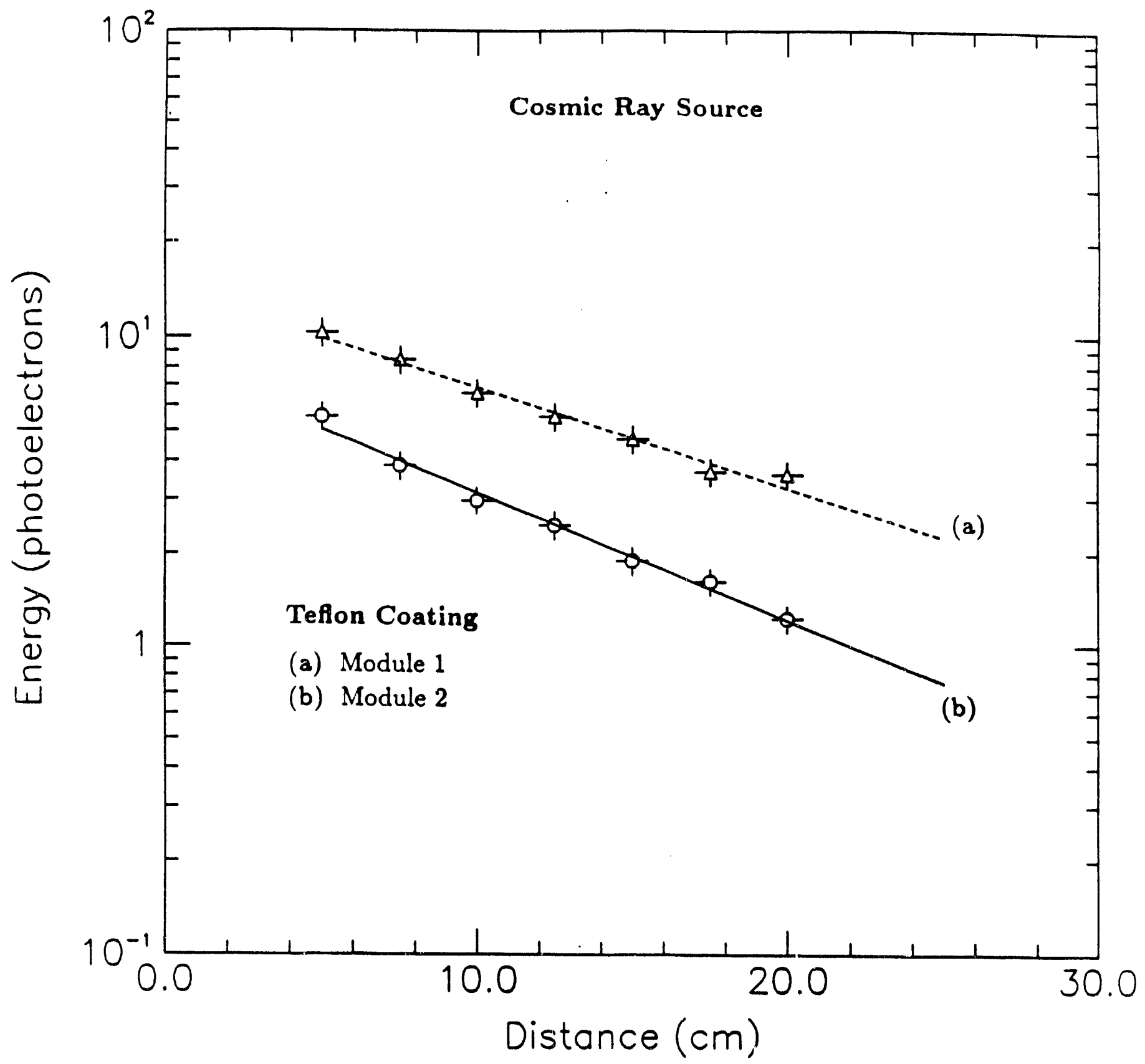

Figure 15. Individual module measurements for Teflon tube coated modules using cosmic rays (muons). 


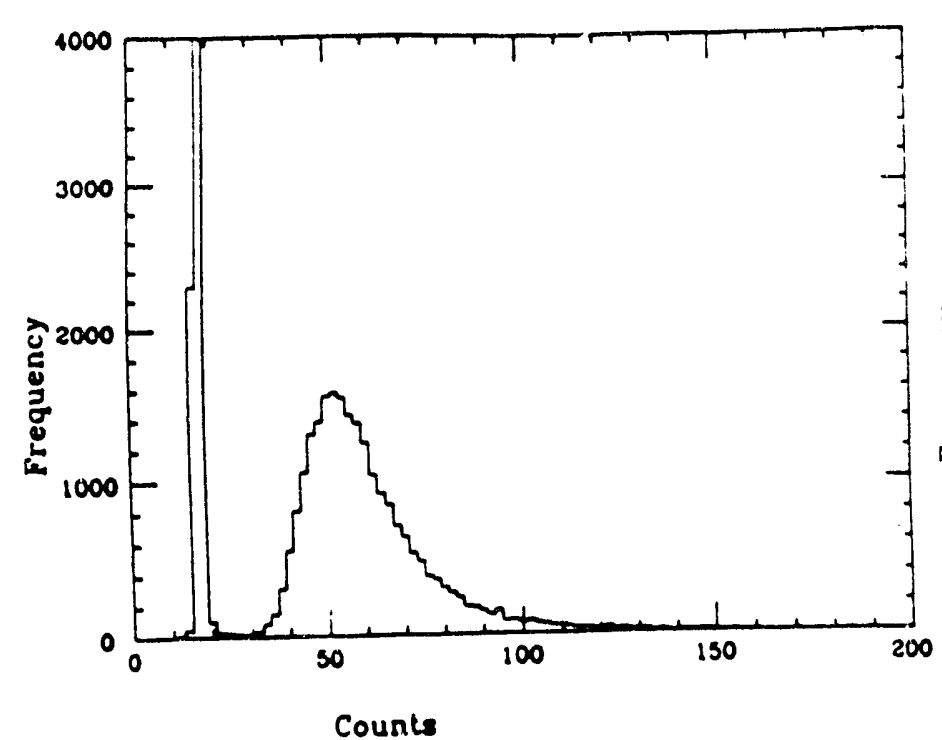

(a) Counter 1

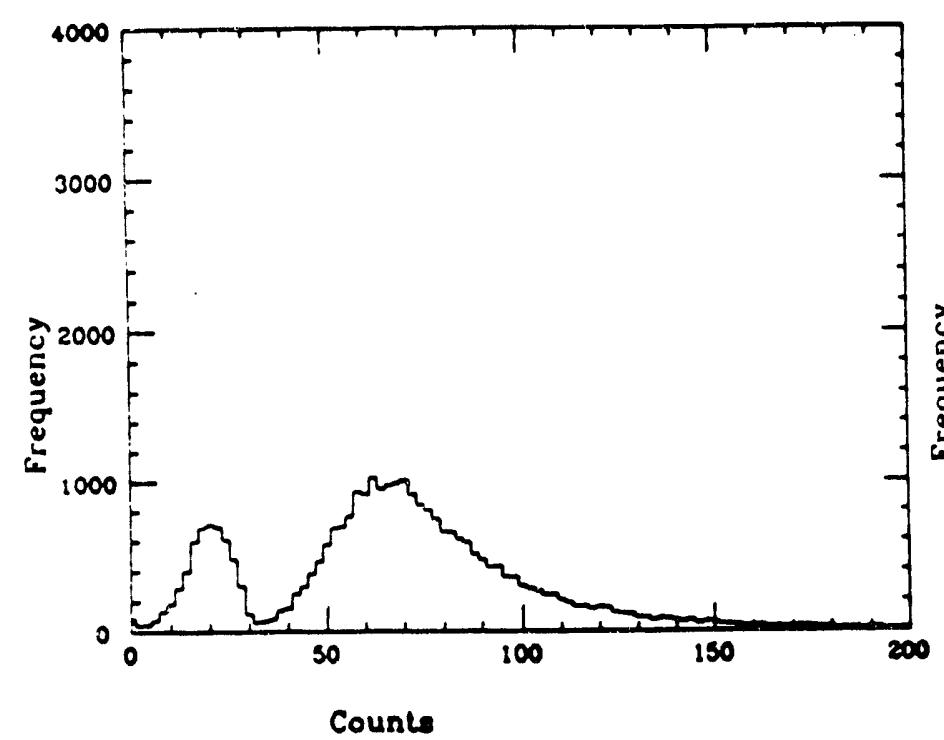

(c) Counter 3

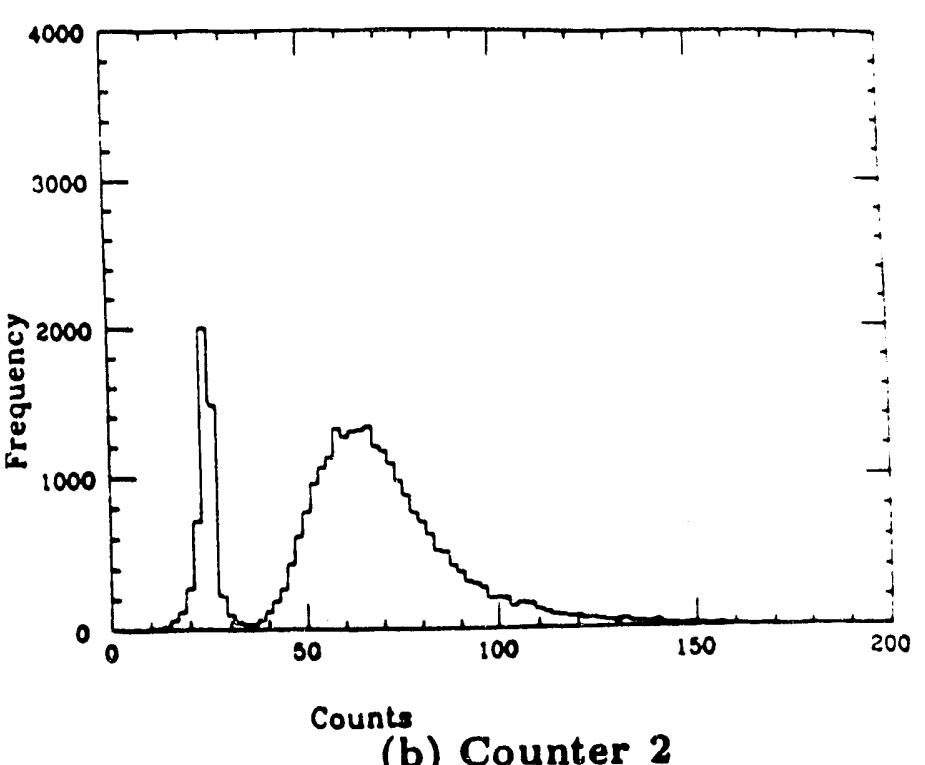

(b) Counter 2

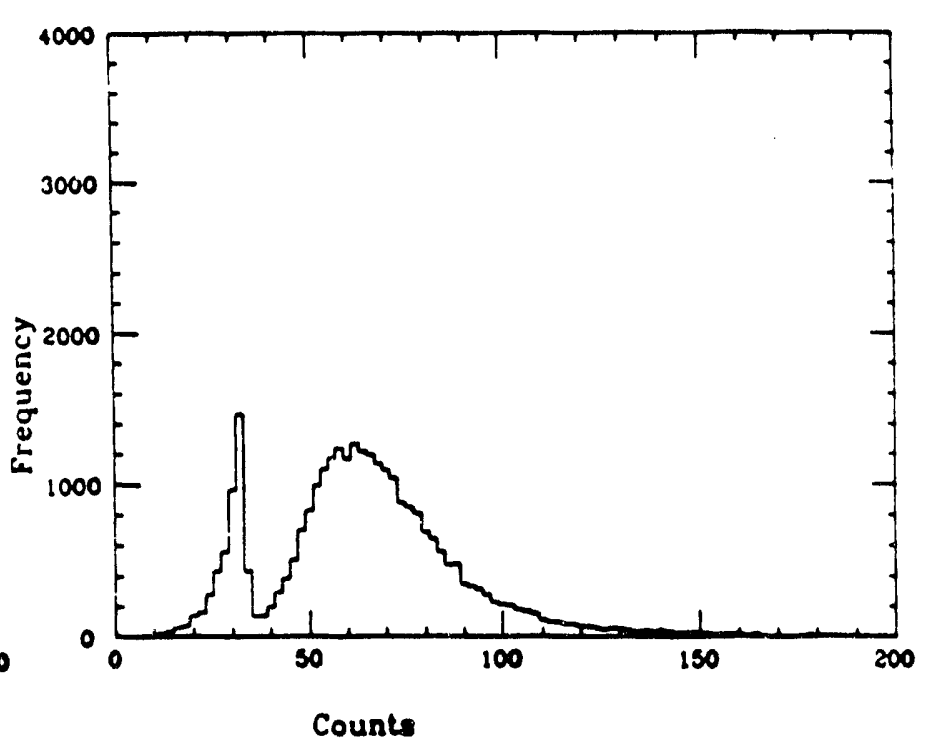

(d) Counter 4

Figure 16. Characteristic spectra of the paddle counters in the close region with cosmic rays (muons). 


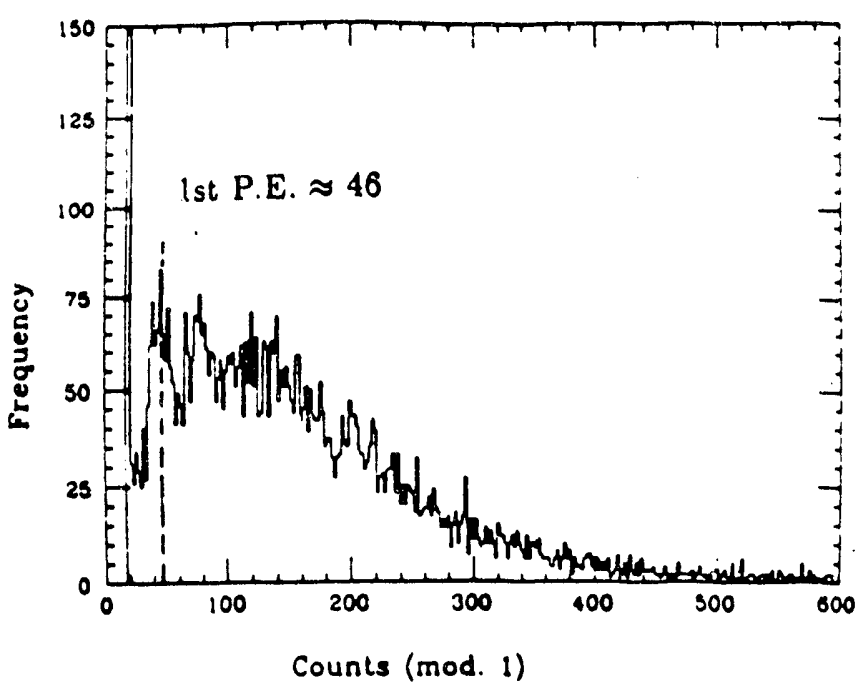

(a)

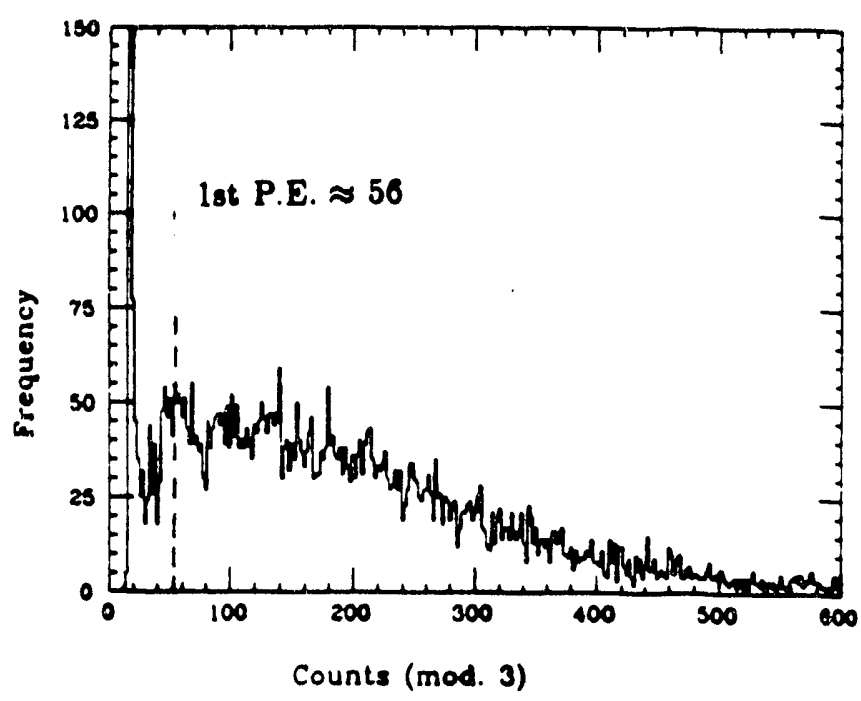

(c)

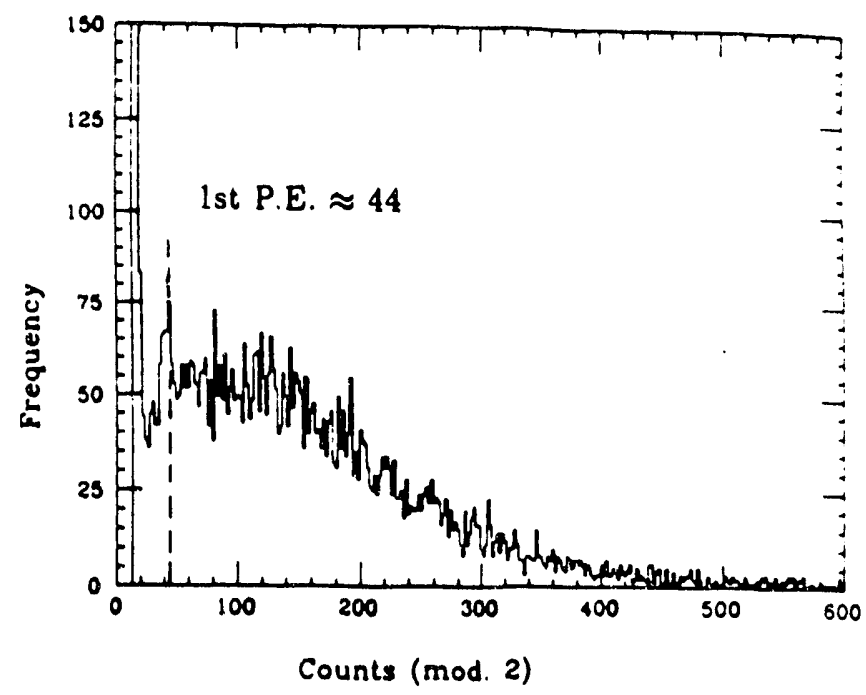

(b)

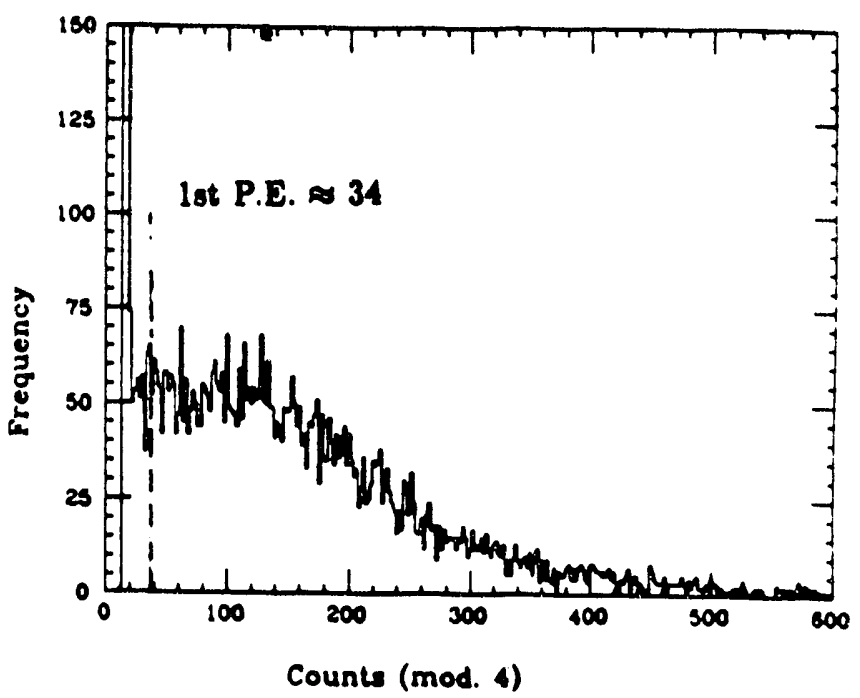

(d)

Figure 18(a)-(d). Spectra of Modules 1-4 for the close region with cosmic rays (muons). The 1st photoelectron (P.E.) position is discernible in each of the spectra. 


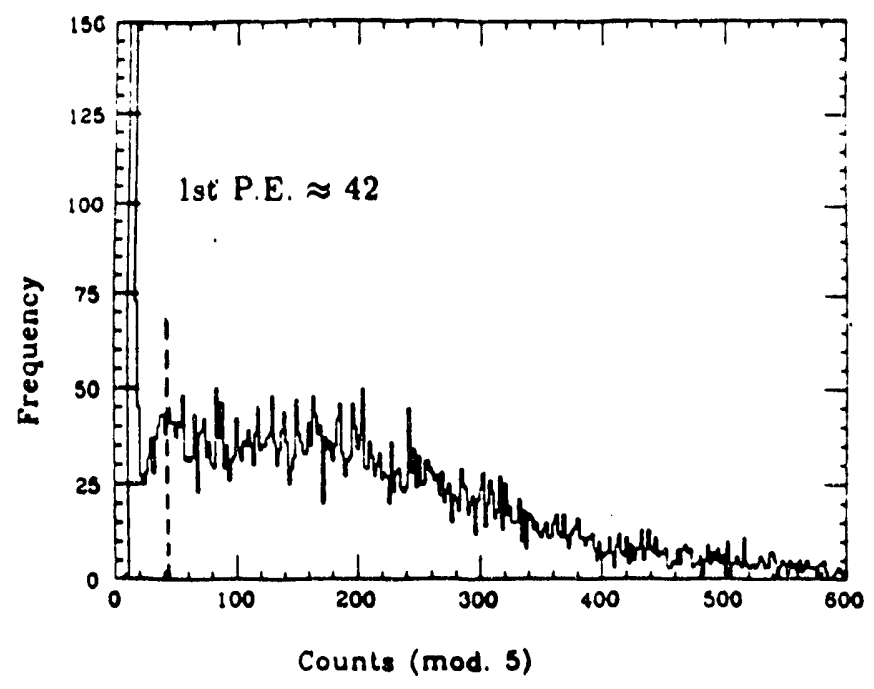

(e)

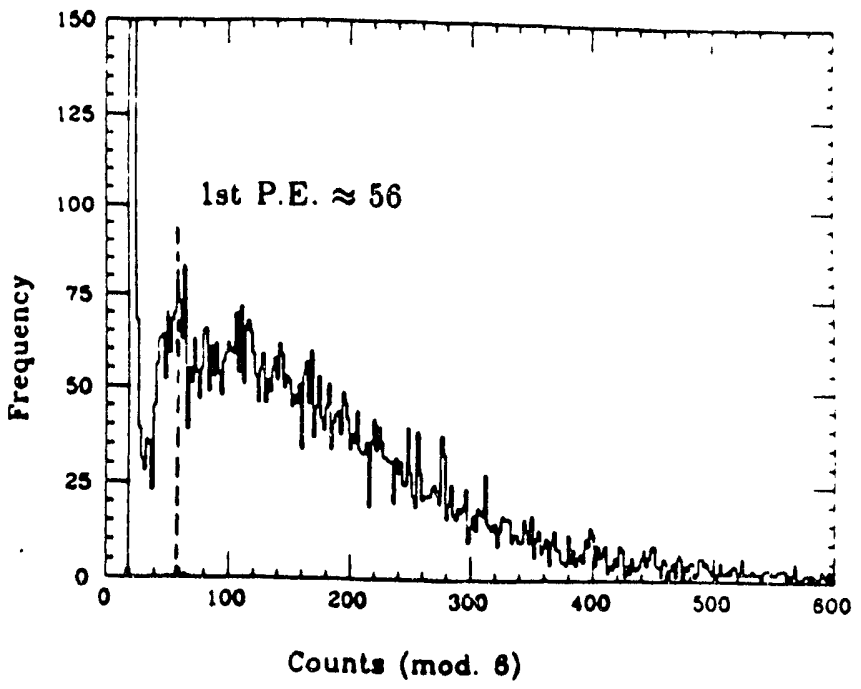

(f)

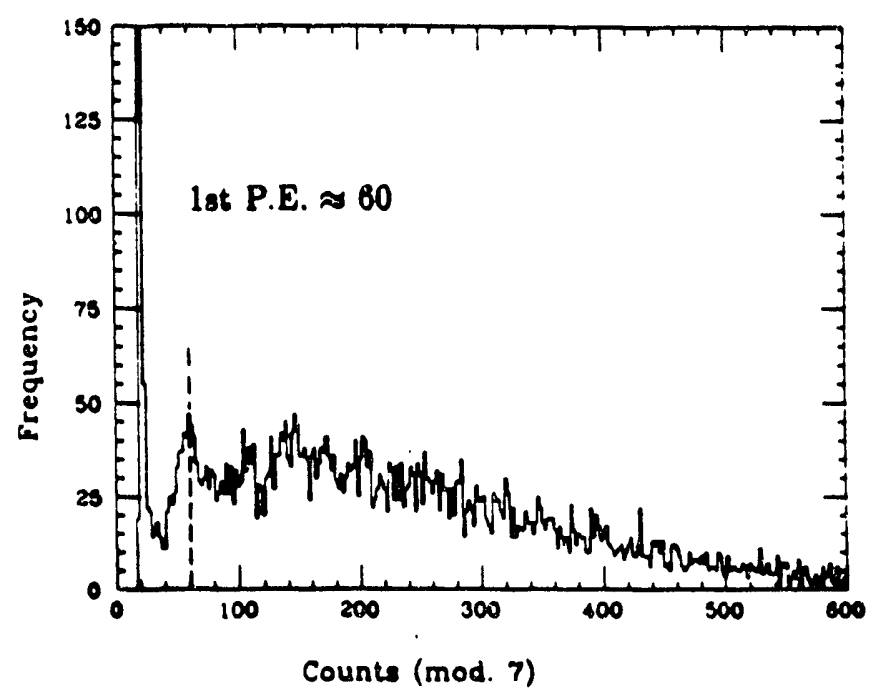

(g)

Figure 18(e)-(g). Spectra of Modules 5-7 for the close region with cosmic rays (muons). The 1st photoelectron (P.E.) position is discernible in each of the spectra. 


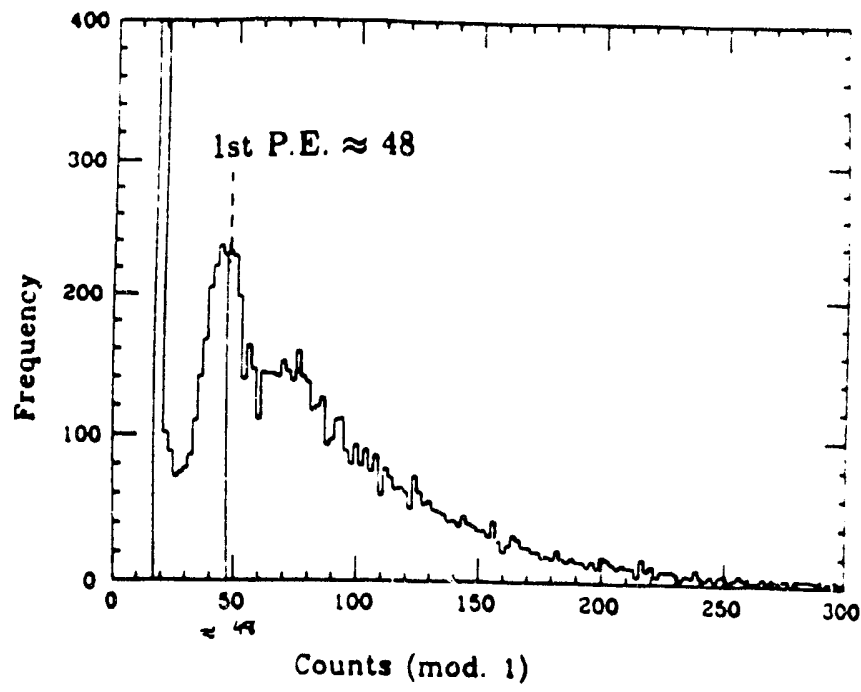

(a)

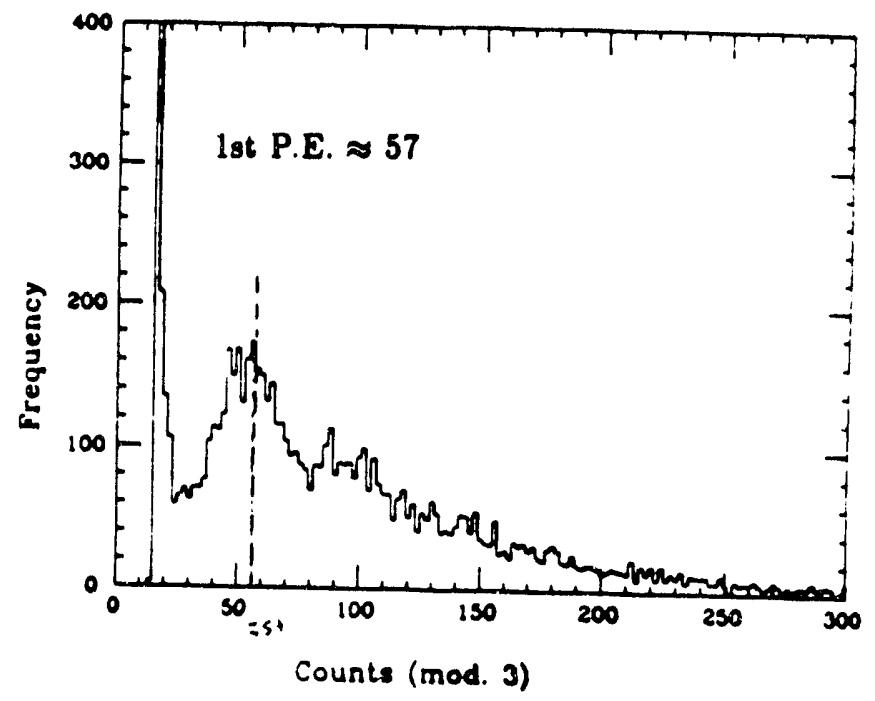

(c)

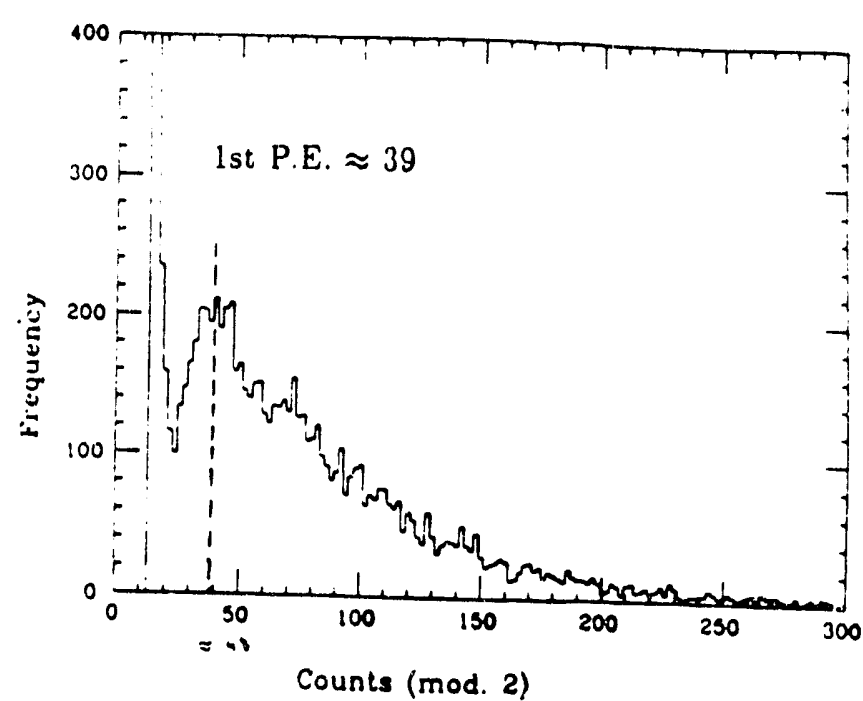

(b)

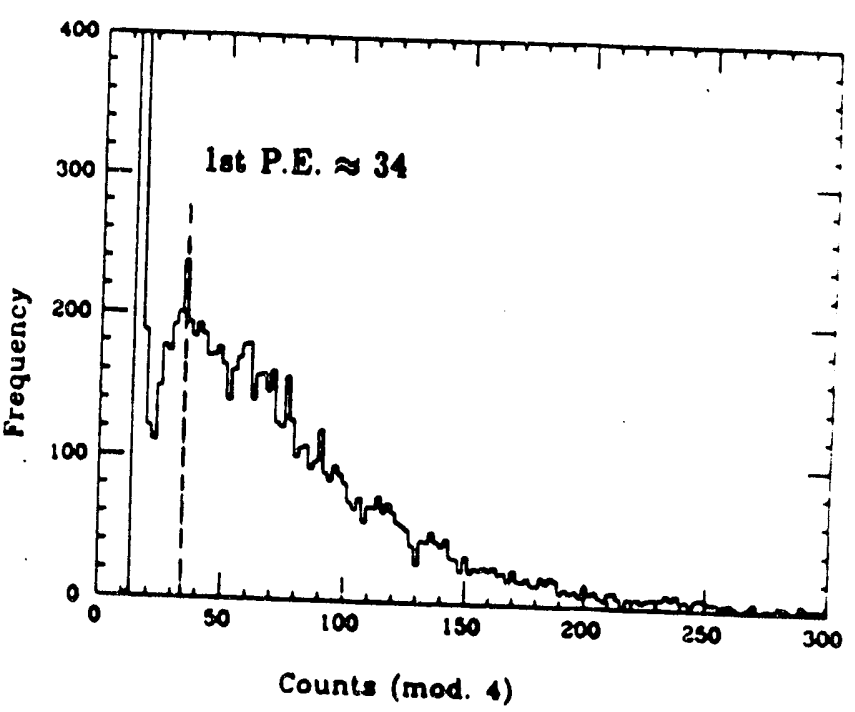

(d)

Figure 19(a)-(d). Spectra of Modules 1-4 for the far region with cosmic rays (muons). The 1st photoelectron (P.E.) position is discernible in each of the spectra. 


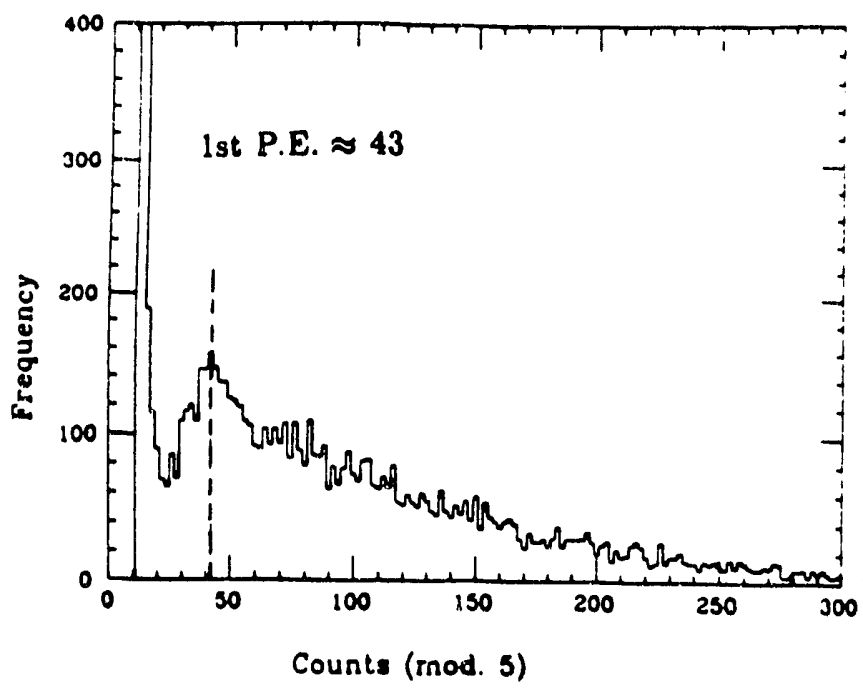

(e)

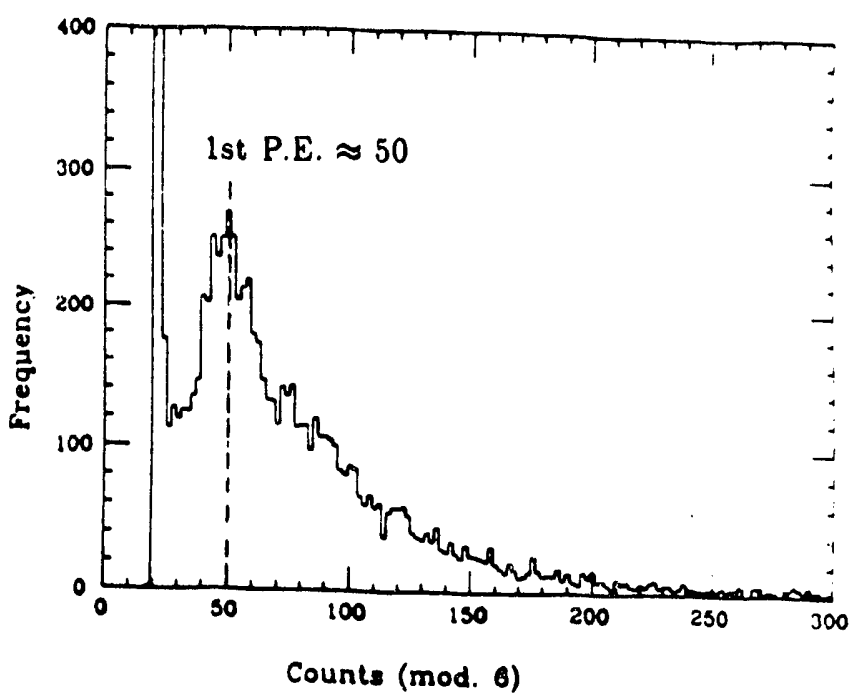

(f)

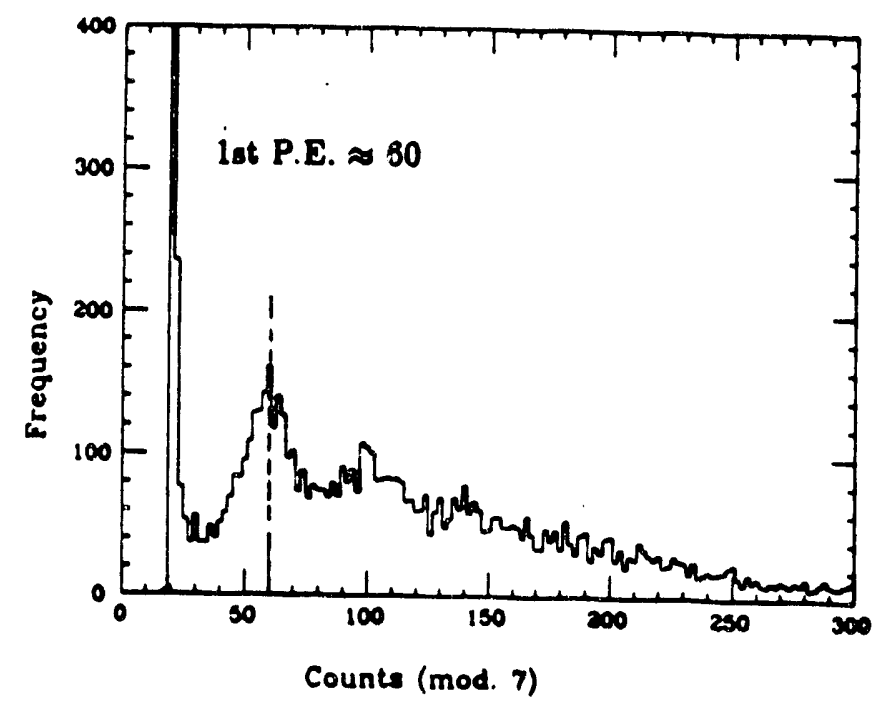

(g)

Figure 19(e)-(g). Spectra of Modules 5-7 for the far region with cosmic rays (muons). The 1st photoelectron (P.E.) position is discernible in each of the spectra. 

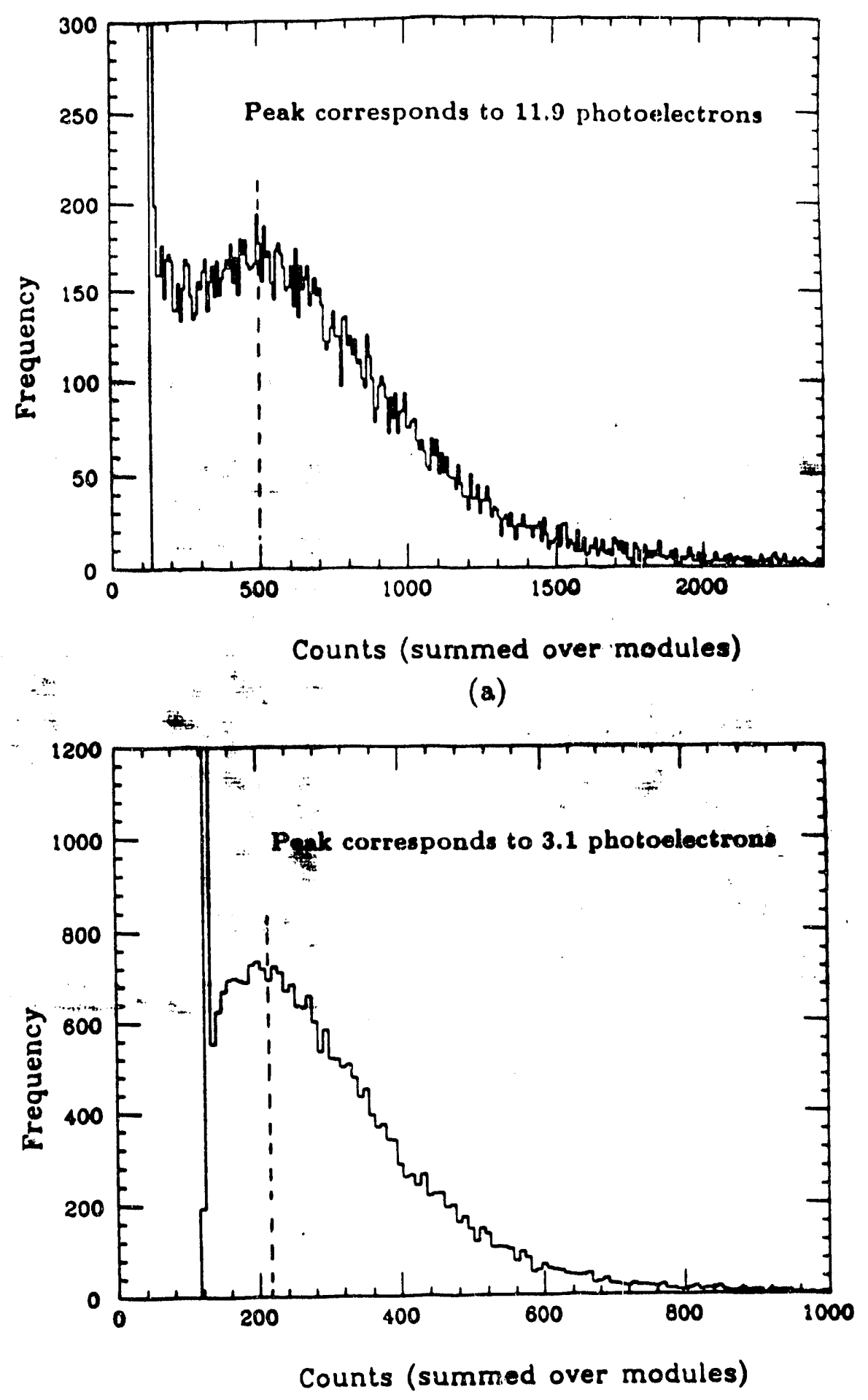

(b)

Figure 20. (a) Results of the close region summed over modules. (b) Results of the back region summed over modules.

(One photoelectron corresponds to 30 counts). 


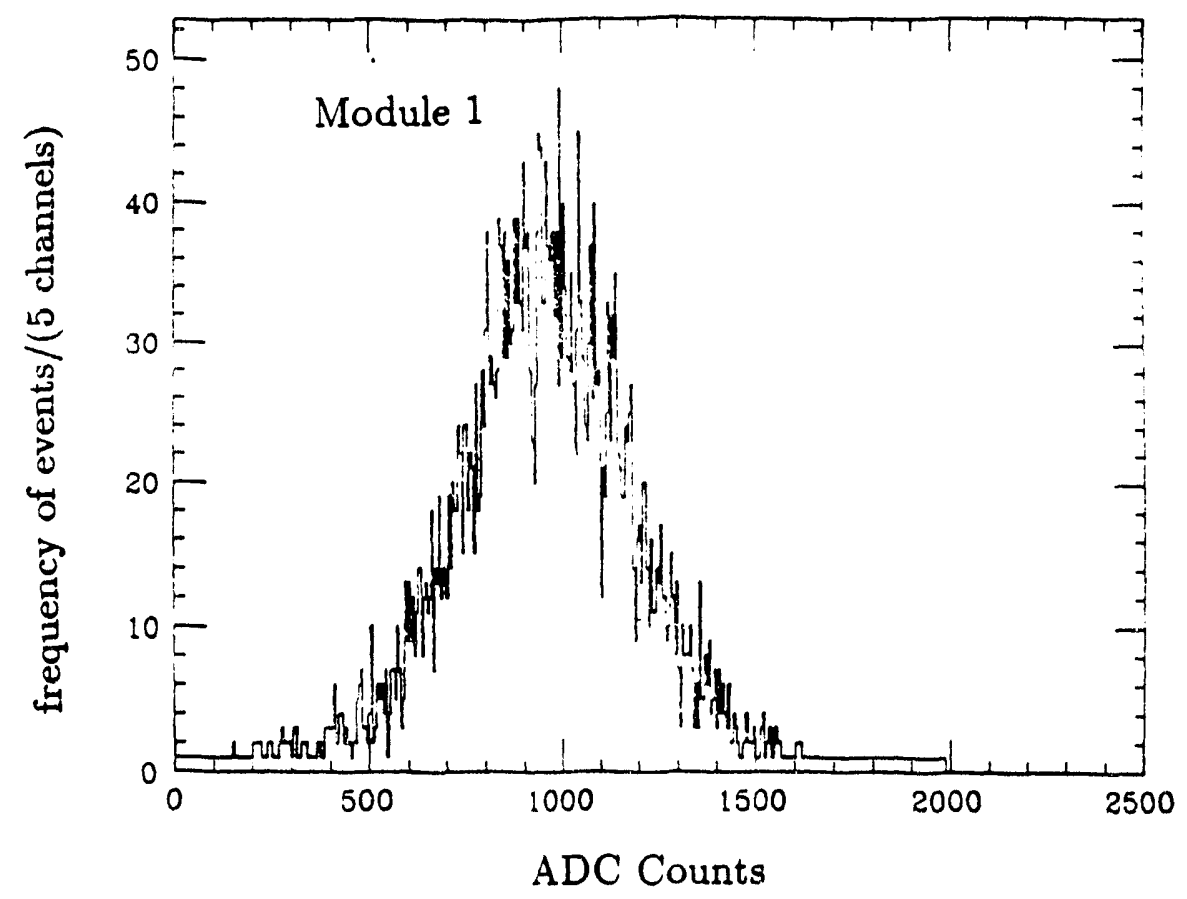

(a)

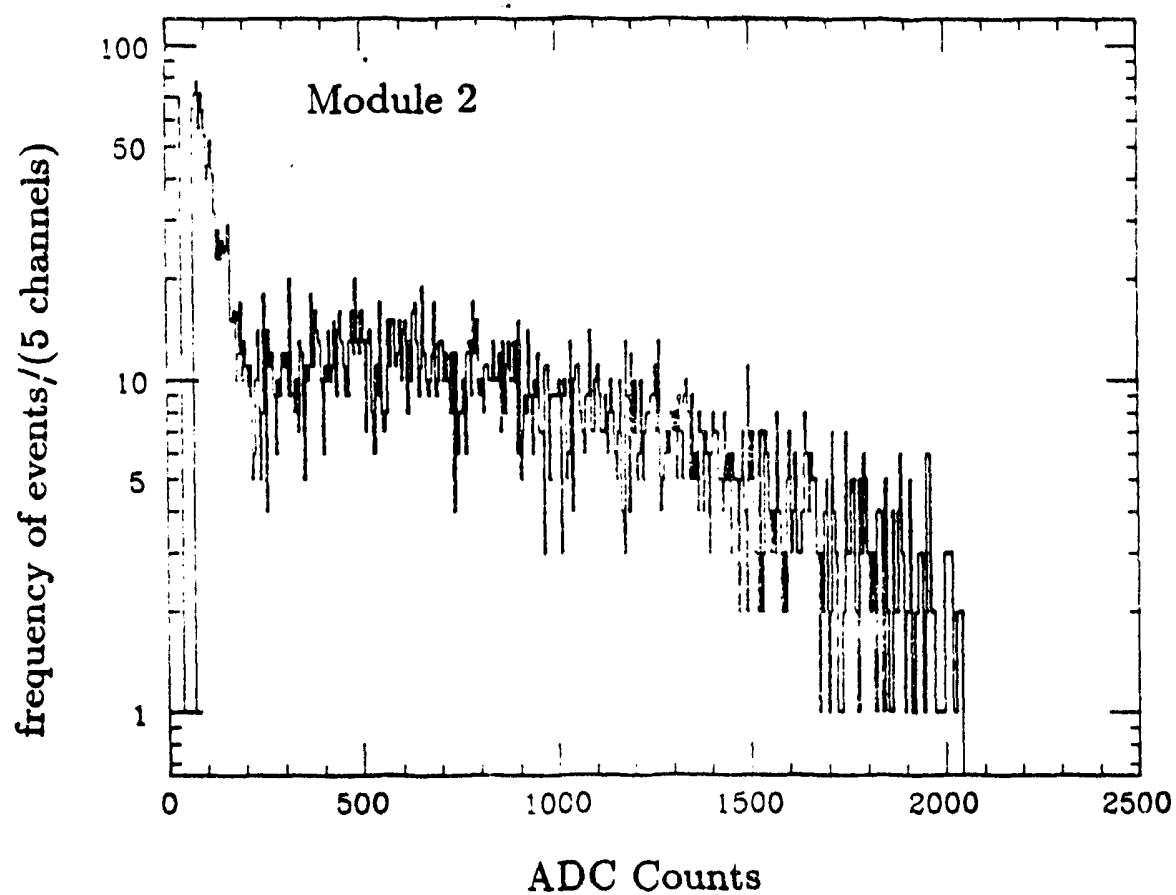

(b)

Figure 21(a),(b). Spectra of Modules 1(Middle) and 2(back) with $10 \mathrm{GeV} e^{-}$beam. 


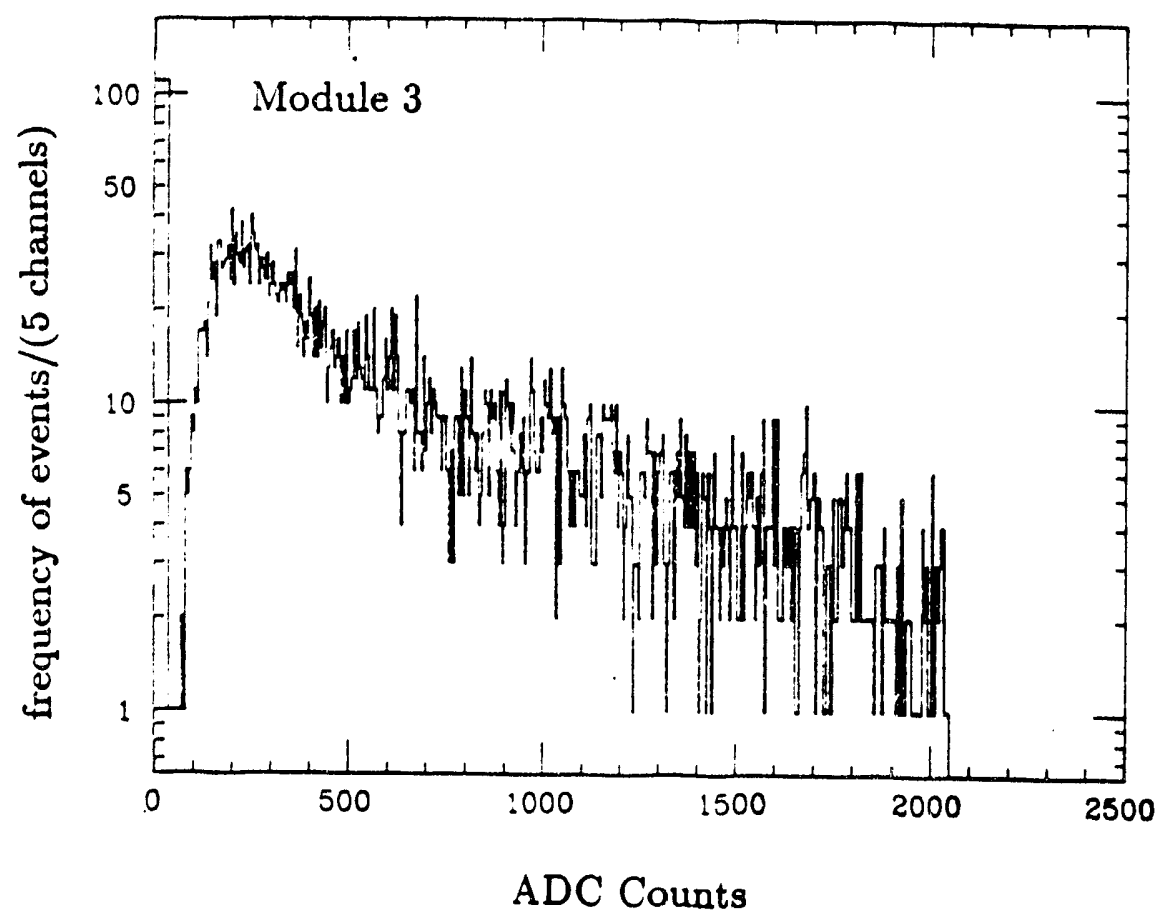

(c)

Figure 21(c). Spectra of Modules 3(front) with $10 \mathrm{GeV} e^{-}$beam. 


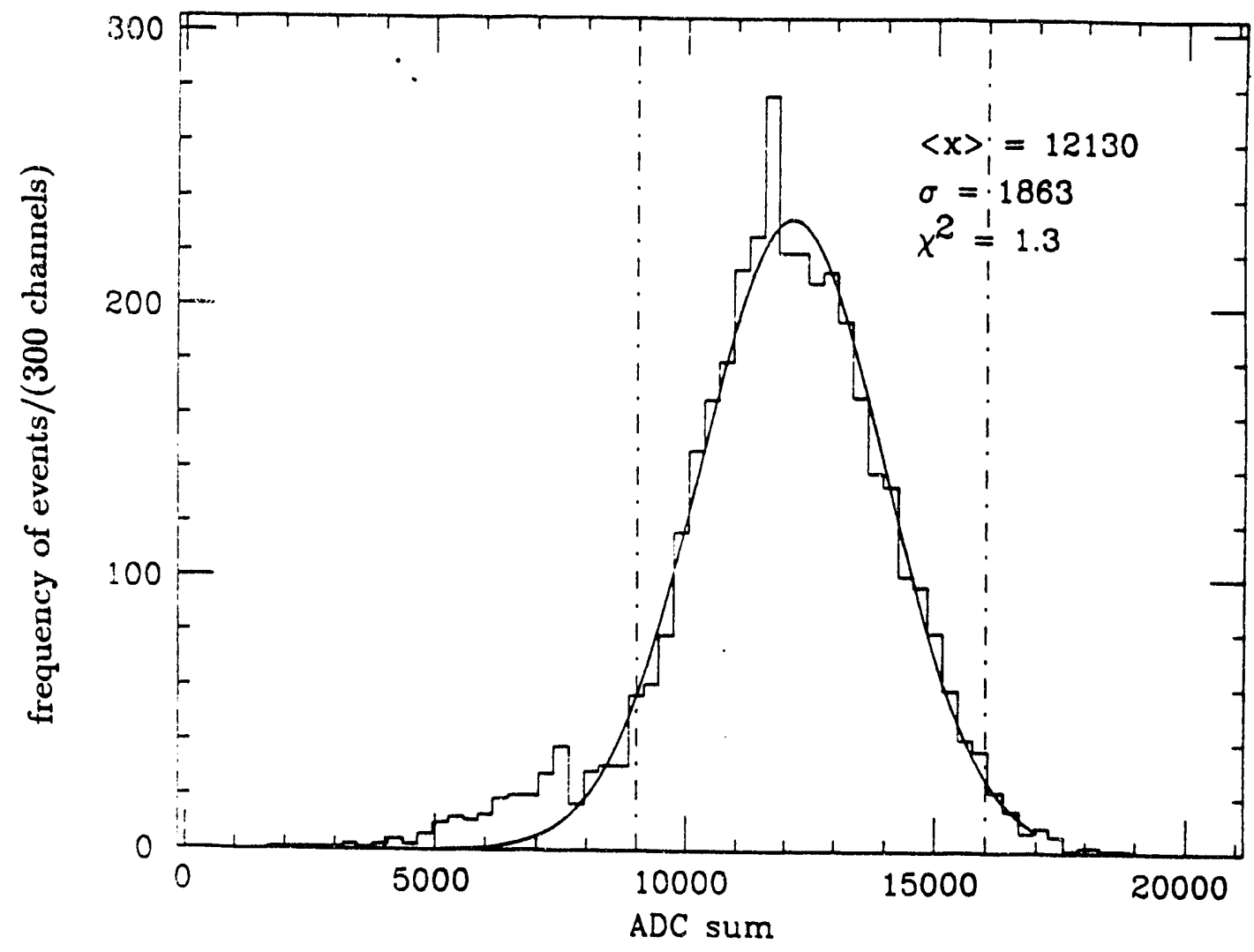

Figure 22. Spectra of sum of the ADC counts of Modules 1 - 5 with $10 \mathrm{GeV}$ $e^{-}$beam. 


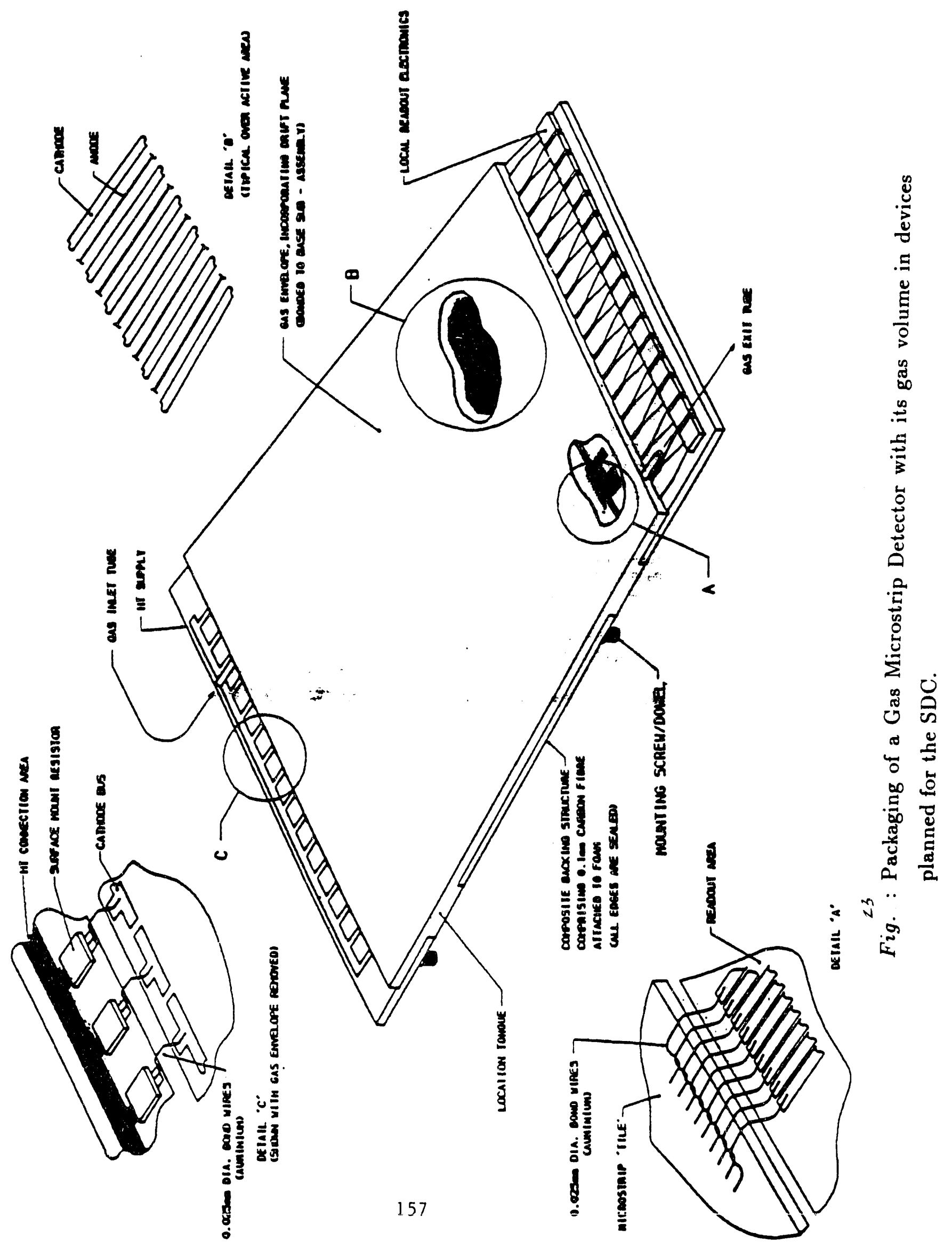



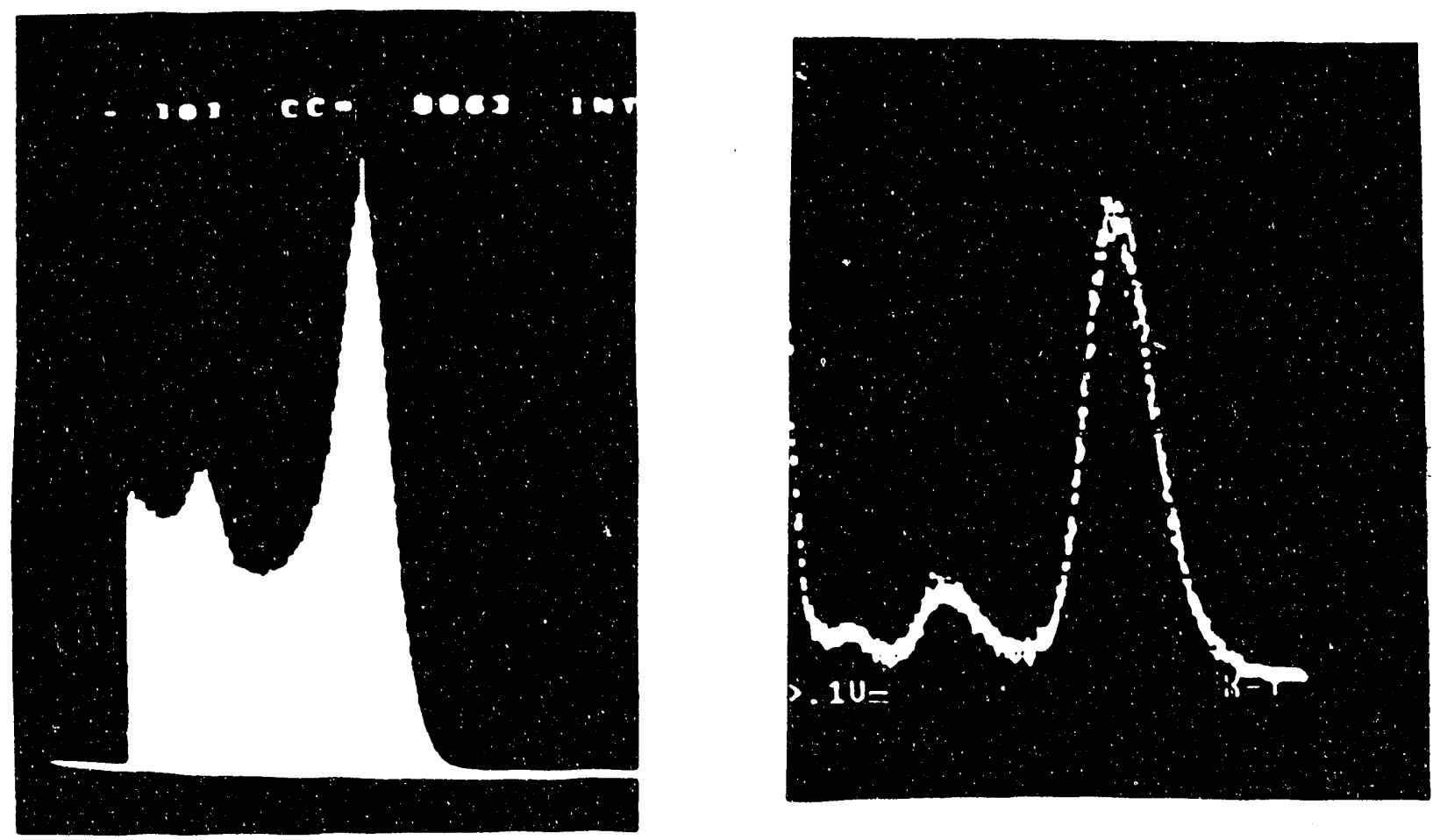

\footnotetext{
24

Fig. : Spectra obtained from ${ }^{55} \mathrm{Fe}$ on various substrates from GMD devices built at Texas A\&M and Purdue. In each of these devices, the photopeak and Argon escape peak are clearly separated. Resolution is typically $20 \%$ FWHM at $10^{3}$ gain. Left: chamber using Upilex S plastic (Texas A\&M), right: chamber using crown glass (Purdue).
} 


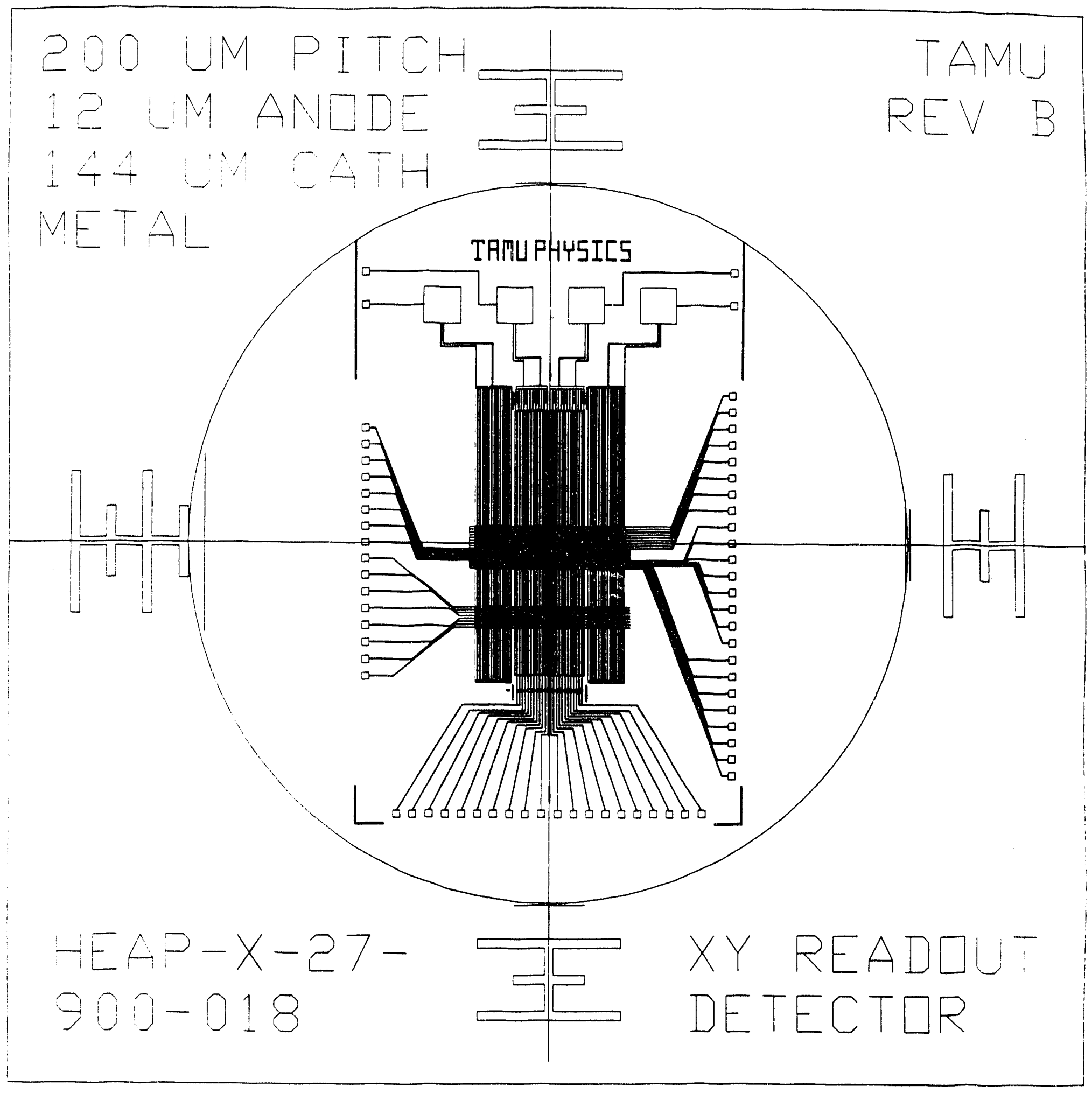

Fig $^{25}$ a : A two-dimensional mask set pattern planned for use in the manufacture of multi-layer GMD devices in the program proposed here. 

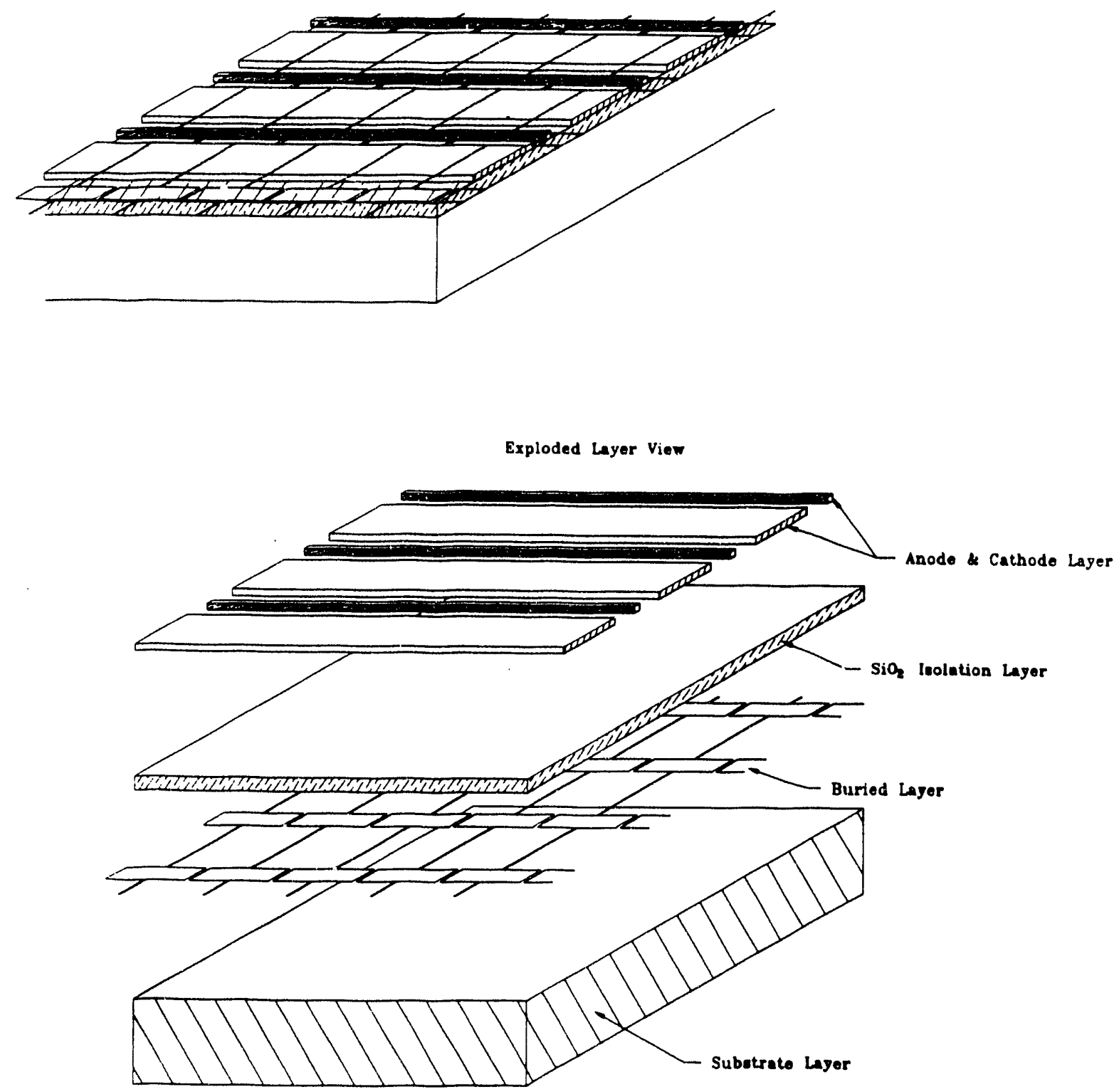

25

Fig. $\quad$ : : Perspective view showing a closeup of the trace pattern in this design. 


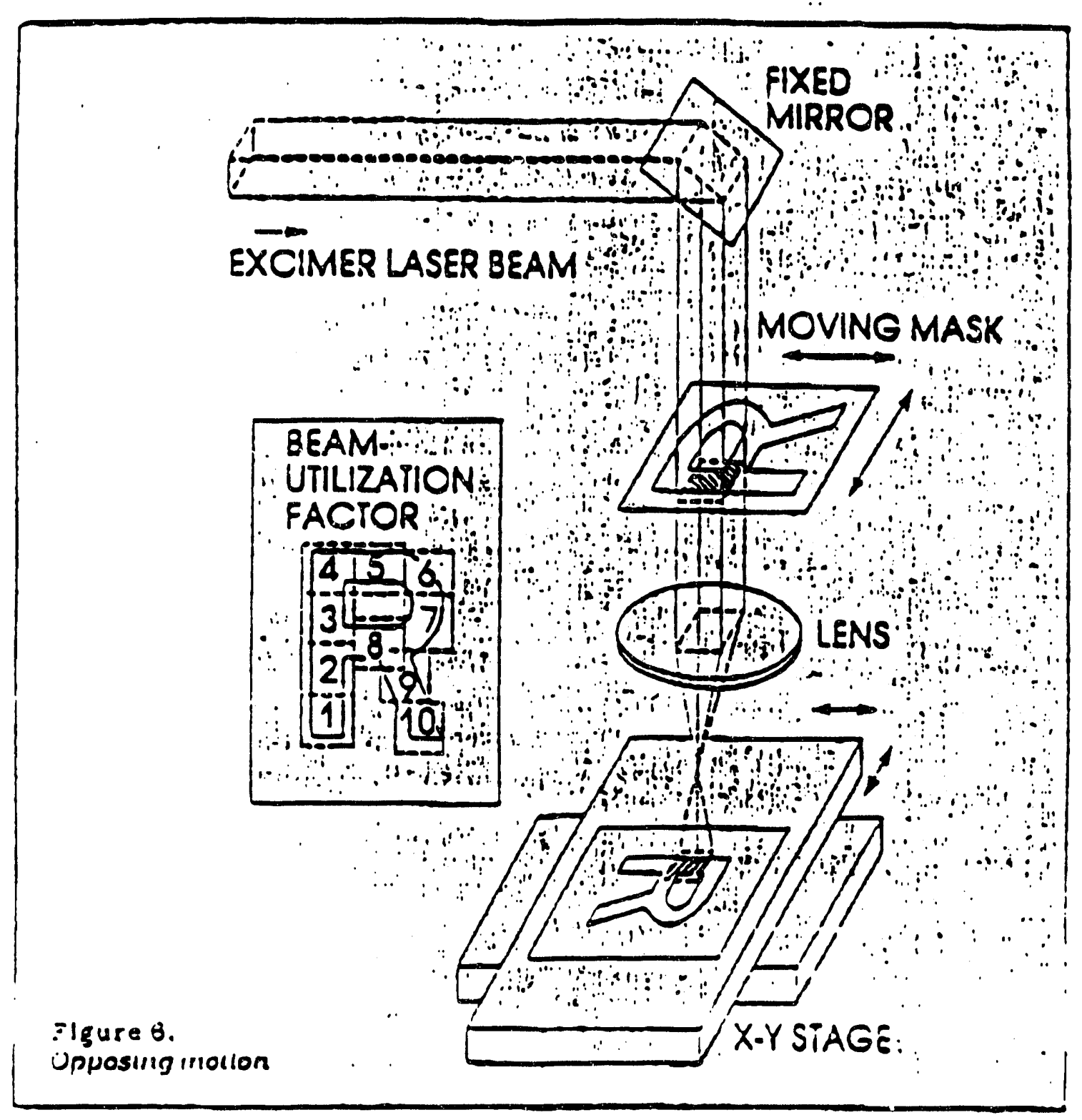

Fig. $^{26}$ : Scanned mask/image machining scheme used to pattern or directly etch a photoresist or metal by the technique of excimer lithography. 


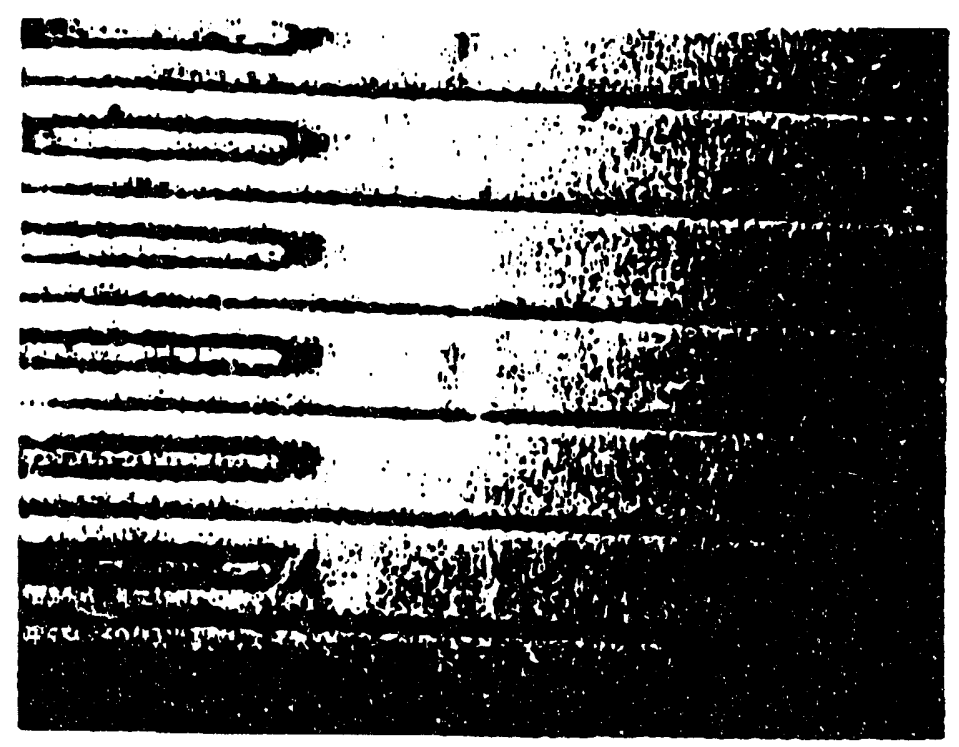

Fig. ${ }^{27}$ : A GMD pattern produced by excimer laser ablation in $0.5 \mu m$ thick photoresist. 


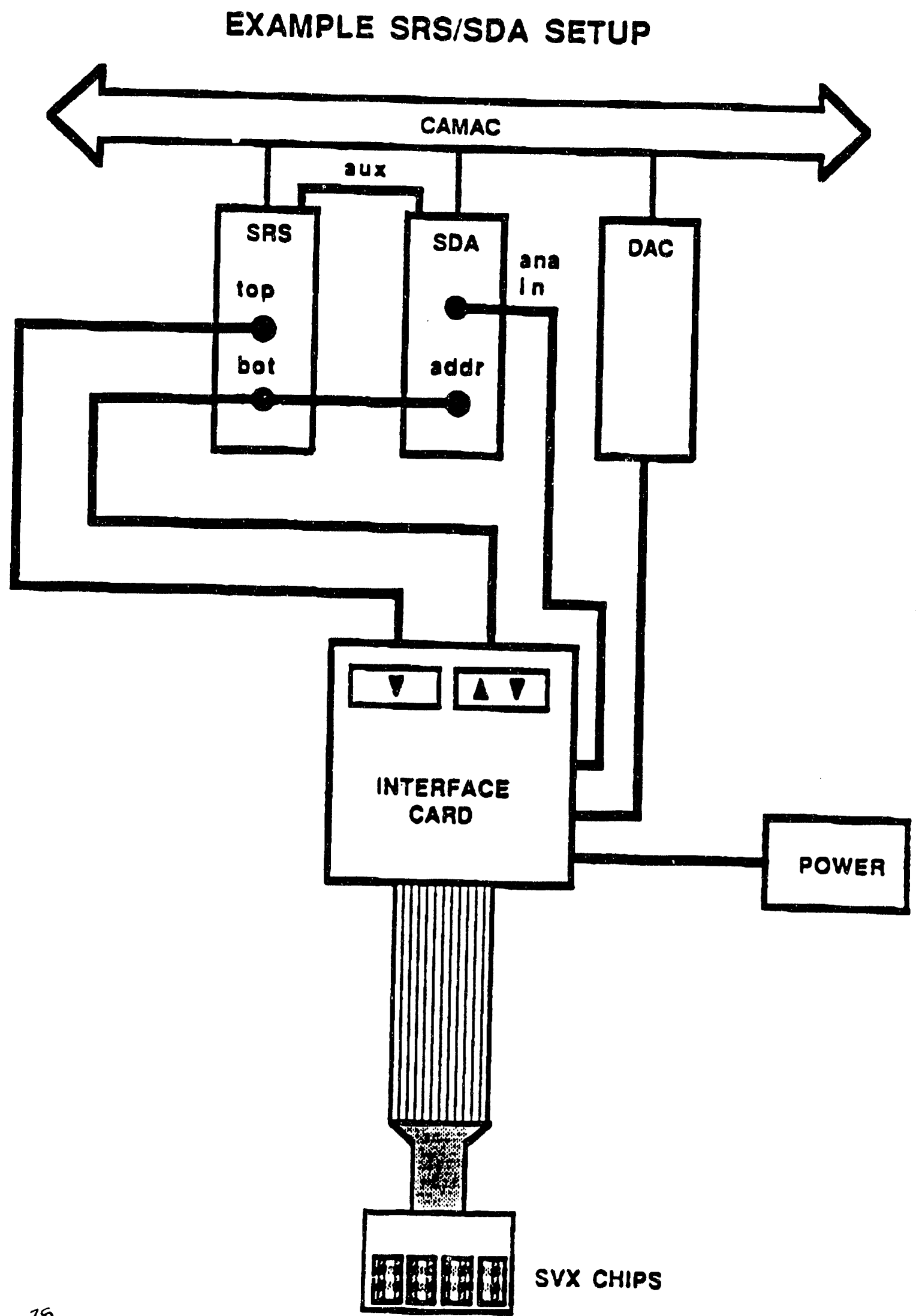

$F_{i g}{ }^{28}:$ Test setup for GMD readout using the SVX Silicon Readout System / Silicon Data Ácquisition (SRS/SDA) system. 


\section{Theoretical Physics Program}

Theoretical high-energy physics research is being carried out by Principal Investigators Professors D.V. Nanopoulos and C.N. Pope. In the section that follows we present a report of the activities that these two researchers have carried out under this program, and their plans for the coming funding cycle. Also included is a report of the research activity of the DOE-funded postdoc, Dr Heath Pois. C.N. Pope's graduate students H. Lu and X.J. Wang have also been partially funded from the grant during the last year. 
Research by D. V. Nanopoulos During 1991-92 issues:

My research during the past academic year has focused on three major

1) SUSY Phenomenology

2) Astroparticle Fhysics

3) Black Holes and the Origin of Space and Time, in String Theory

I. SUSY Phenonenology

As time goes by, Supersymmetric (SUSY) Theories seem to be the only viable, natural extension of the Standard Model (SM). Two years ago we first pointed out [1] that the precise LEP data on $\alpha_{3}$ and $\sin ^{2} \theta_{w}$ offer tantalizing evidence for the unification of strong and electroweak interactions, if and only if one supersymetrizes the SM. Since then SUSY has become again the focus of intense phenomenological investigations. With my group at Texas $A \& M$, we have developed a very comprehensive, systematic program for studying all issues related to SUSY. Working in the framework of spontaneously broken Supergravity, which corresponds to softly broken supersymmetry, we have been able to reduce immensely the available parameter space and work out interesting correlations among the plethora of supersymmetric masses. In the standard framework of supergravity theories, electroweak breaking may occur radiatively, thus providing a dynamical explanation why $M_{W}<<M_{P l a n c k}$ [2]. Furthermore, in the framework of no-scale supergravity [2], the infrared limit of the string, the arbitrary parameters are reduced to a minimal choice, although still acceptable, and testable models emerge. Our work is contained in [3-10]. As a measure of the power of our approach, let me mention the case of the minimal SU(5) supergravity, where we have proven [1C] that either [10] (i) the lightest chargino has to be below $100 \mathrm{GeV}$ (i.e. observable at Fermilab) or (ii) the lightest Higgs has to be below $50 \mathrm{GeV}$ (i.e. observable at LEP I-II) and that in either case $\mathrm{p} \rightarrow \overline{i \mathrm{~K}^{+}}$should be observed at Gran Sasso/SuperKamiokande at a fraction of their sensitivity. It look like we are going to know very soon about the fate of minimal supergravity $\mathrm{SU}(5)$.

In the model building front, variations of the flipped SU(5) have been presented [11-15], where by enlarging the low energy content of the theory by $10+\overline{10}$ multiplet(s), the unification of coupling constants naturally occurs at $10^{18} \mathrm{GeV}$, as expected in string unification $[16,17]$. 


\section{Astroparticle Physics}

As time goes by, it becom clear that the most promising candidate for Dark Matter (DM) is the neutralino (some linear combination of the superpartners of $\left.\gamma, \mathrm{Z}, \mathrm{H}^{0}, \overline{\mathrm{H}}^{0}\right)$. We have developed a program at Texas A\&M where we can calculate the cosmic neutralino relic density for SUSY models that automatically satisfy all other particle physics constraints $[18,19,20]$. Interestingly enough, we found that there are regions of the parameter space where the neutralinos can close the universe. One exception is the minimal supergravity SU(5) where only very few points are acceptable and that is the reason for the super-fine correlations mentioned in the previous section. DM detectors will tell us soon about the viability of neutralinos as a DM candidate.

On a slightly different wavelength, we have made use of the recent results from COBE and GALLEX and have concluded that a 708 cold DM (e.g. neutralinos) and a 308 hot DM (e.g. neutrinos) mixture can explain everything. We have worked out [21] a very detailed, flipped SU(5) inspired neutrino mass spectrum that fits all avaliable solar neutrino and cosmological data and predicts observable neutrino oscillations at forthcoming experiments at CERN, i.e. CHORUS and NOMAD. Let me stress that during the last year both in SUSY phenomenology and Astroparticle physics fronts, experimental data and correlations have been refined to such a point that we may see soon, and definitely before the SSC, the refutation or confirmation of SUSY, at least as it is described by a large class of models.

\section{Black Holes and the Origin of Space and Tine in String Theory}

A major part of my research time during the last year has been devoted to a better understanding of black holes and the origin of space and time in string theory. In a series of papers [22-24], we have presented a completely new picture describing black holes, space time foam, and the origin of space-time. We also have found some profound changes in quantum mechanics, as 'observed' at low energies ( $<\ll M_{P l a n c k}$ ) that may be responsible for the collapse of the wavefunction [23].

The key point of the whole picture is the existence of an infinite set of new symmetries, W-symmetries whinh characterize the string in curved space-time. This $\mathrm{W}_{1+\infty}$-symmetry contains an infinite set of Cartan sub-algebras which commute with the Hamiltonian, thus implying an infinite set of 'measurable' charges. This complete set of infinite, measurable W-charges 
characterizes string black holes and thus enables them to restore and transmit information in a coherent way. There is no transition from a pure to mixed state, i.e. there is no Hawking radiation. Black holes decay like normal massive string modes, in a coherent way. In the limit that we sum over $W$-charges, we recover the Hawking-Beckenstein entropy formula $S_{B H} \sim M_{B H}^{2}$. In string theory black holes are 'normal' massive states, and the Hawking radiation looks like the results of inadequate semiclassical approximations analogous to the Rutherford radiation in the classical aton. Furthermore, we have provided a complete description of the 'classic singularity' in terms of Witten's Topological Field Theory (TFT), and we have found that the environment near a black hole or cosmic singularity is isomorphic to a quantum Hall fluid!!! Thus, studying the Quantum Hall Effect (via table-top experiments) we can learn a lot about cosmic singularities and their environments, space-time foam and the inner workings of black holes. Already we are developing collaborations both with experimental and theoretical condensed matter physicists for future study of this amazing connection.

As a byproduct, the origin of space and time seems to be a phase transition that takes us from $W \times W$ symmetry down to the $W$-symmetry responsible for quantum coherence! Because the $W$-symmetry, responsible for quantum coherence links light with massive ( $\mathrm{M}_{\mathrm{P} 1 \text { anck }}$ ) string states, and a low-energy observer only 'sees' the light states, we may end up with an effective quantum mechanics where quantum coher ance is lost. Actually, because of the space-time foam which acts as a quantum-gravitational friction term, the "light" systems behave like "open" systems, with a naturally created cosmic arrow of time, an increase of entropy, and thus bound to collapse! We have worked out some details of this mechanism and found that the center of mass of a body with $\mathrm{N} \sim \mathrm{N}_{\text {Avogadro }}$ constituents is confined within the Bohr radius in $10^{-7}$ seconds! In other words we may have a dynamical mechanism for the collapse of the wave function. We have proposed detailed experiments in the $\mathrm{K}^{0}-\overline{\mathrm{K}}^{0}$ system [24] and SQUIDS [25] to measure these effects. All in all, this part of my research has turned out to be very exciting and seems to have far-reaching consequences in many branches of modern physics.

IV. Future Research

I have presented my most-recent activities in such a way that their future analytic continuation is apparent. I will continue working very effectively at the SUSY phenomenology and astroparticle front where we expect 
soon some imminent answers, interesting whatever they may turn out to be. One problem that I am going to devote more time to is the specific mechanism of SUSY breaking in string theory. Once we have solved this problem, the whole SUSY phenomenology will be pinned down almost completely.

On the black hole/quantum mechanics part I will continue working vig. orously on different fronts: from the connection/isomorphism between black holes and quantum Hall fluids, propose experiments in condensed matter physics to test our ideas; work out in detail a cosmological scenario and propose new tests of the validity of quantum mechanics and checking our "collapse of the wave function" mechanism. 


\section{REFERENCES}

[1] D. Nanopoulos, J. Ellis, and S. Kelley, "Precision LEP Data, Super. symmetric GUTs and String Unification," Phys. Lett. B249, 441 (1990); D. Nanopoulos, J. Ellis, and S. Kelley, "Probing the Desert Using Gauge Coupling Unification," Phys. Lett. B260, 131 (1991); D V. Nanopoulos, J. Ellis, and S. Kelley, "A Detailed Comparison of LEP Data with Predictions of the Minimal supersymmetric SU(5) GUT, "Nucl. Phys. B373, 55 (1992); D. V. Nanopoulos, J. Ellis, and S. Kelley, "Constraints from Gauge Coupling Unification on the Scale of Supersymmetry Breaking," Phys. Lett. B287, 95 (1992).

[2] D. Nanopoulos and A. B. Lahanas, "The Road to No-Scale Supergravity," Physics Reports 145, 1 (1987).

[3] D. Nanopoulos, A. Faraggi, J. Hagelin, and S. Kelley, "Sparticle Spectroscopy," Phys. Rev. D 45, 3272 (1992).

[4] D. Nanopoulos and J. Lopez, "New Theoretical Lower Bounds on the Higgs Sector of Minimal SUSY," Phys. Lett. B266, 397 (1991).

[5] D. V. Nanopoulos, S. Kelley, J. Lopez, H. Pois, and K. Yuan, "No-Scale Supergravity Confronts Experiment," Phys. Lett B272, 423 (1991).

[6] D. V. Nanopoulos, S. Kelley, J. Lopez, H. Pois, and K. Yuan, "Higgs Boson Masses in No-Scale Supergravity," Phys. Lett B285, 61 (1992).

[7] D. V. Nanopoulos, S. Kelley, J. Lopez, H. Pois, and K. Yuan, "Aspects of Radiative Electroweak Breaking in Supergravity Models," TAMU preprint CTP-TAMU-16/92, ACT-5/92, CERN-TH-6498/92.

[8] D. V. Nanopoulos, J. Lopez, and A. Zichichi, "Troubles with the Minimal SU(5) Supergravity Model," Phys. Lett. B291, 255 (1992).

[9] D. V. Nanopoulos, J. Lopez, and H. Pois, "Proton Decay and Cosmology Strongly Constain the Minimal SU(5) Supergravity Model," CTP-TAMU61/92, ACT-19/02 CERN-TH-6628/92.

[10] J. Lopez, D. V. Nanopoulos, H. Pois, and A. Zichichi, "Proposed Tests for Minimal SU(5) Supergravity at Fermilab, Gran sasso, Super Kamiokandi, and LEP," CERN-TH-6716/92, CTP-TAMU-72/92. ACT-21/92.

[11] D. V. Nanopoulos, I. Antoniadis, J. Ellis, and S. Kelley, "The Price of Deriving the Standard Model From String," Phys. Lett. B272, 31 (1991). 
[12] D. V. Nanopoulos and J. Lopez, "Flipped SU(5): Origins and Recent Developments," in the Proceedings of the 15th Johns Hopkins Workshop on Current Problems in Particle Theory, August 1991, p. 277; ed. by G. Domokos and S. Kovesi-Domokos (World Scientific, 1992).

[13] D. V. Nanopoulos, S. Kelley, and J. Lopez, "A New String-Inspired Standard Model (SISM)," Phys. Lett. B278, 140 (1992).

[14] D. V. Nanopoulos, J. Lopez and K. Yuan, "The Search for a Realistic F1ipped SU(5) String Model," TAMU preprint CTP-TAMU-11/92, ACT-1/92.

[15] D. V. Nanopoulos, J. Lopez, and.A. Zichichi, "The Simplest, String-derivable, Supergravity Model and its Experimental Predictions," TAMUpreprint CTP-TAMU-68/92, ACT-20/92, CERN-TH-6667/92.

[16] D. V. Nanopoulos, I. Antoniadis, J. Ellis and R. Lacaze, "String Threshold Corrections in Flipped SU(5)," Phys. Lett. B268, 188 (1991).

[17] D. V. Nanopoulos, S. Kalara and J. Lopez, "Threshold Corrections and Modular Invaricnce in Free Fermionic Strings," Phys. Lett. B269, 84 (1991).

[18] D. Nanopoulos, J. Lopez, and K. Yuan, "SUSY GUTs Dark Matter," Nucl. Phys. B370, 445 (1992).

[19] D. Nanopoulos, J. Lopez and K. Yuan, "Supersymmetry Breaking and Dark Matter," Phys. Lett. B267, 219 (1991).

[20] D. V. Nanopoulos, S. Kelley, J. Lopez, H. Pois, and K. Yuan, "New Constraints on Neutralino Dark Matter in the Supersymmetric standard Mode1," TAMU-preprint CTP-TAMU-56/92, ACT-16/92, CERN-TH-6584/92.

[21] D. V. Nanopoulos, J. Ellis, and J. Lopez, "The Prospects for CHORUS and NOMAD in the Light of COBE and GALLEX," Phys. Lett. B292, 189 (1992).

[22] D. V. Nanopoulos, J. Ellis and N. Mavromatos, "Quantum Coherence and Two-dimensional String Black-Holes," Phys. Lett. B267, 465 (1991); D. V. Nanopoulos, J. Ellis and N. Mavromatos, "On the Connection Between Quantum Mechanics and the Geometry of Two-dimensional Strings," Phys. Lett. B272, 262 (1991); D. V. Nanopoulos, J. E1lis, and N. Mavromatos, "On the Evaporation of Black Holes in String Theory," Phys. Lett. B276, 56 (1992); D. V. Nanopoulos, J. Ellis, and N. Mavromatos, "Quantum Mechanics and Black Holes in Four-Dimensional string Theory," Phys. Lett. B278, 246 (1992); D. V. Nanopoulos, J. E1lis, and N. Mavromatos, "Measuring the W-Hair," Phys. Lett. B284, 27 (1992); D. V. 
Nanopoulos, J. Ellis, and N. Mavromatos, "Measuring the W-Hair of String Black Holes," Phys. Lett. B284, 43 (1992); D. V. Nanopoulos, J. Ellis, and N. Mavromatos, "Quantum Coherence and String Black Holes," General Relativity essay; D. V. Nanopoulos, J. Ellis, and N. Mavromatos, "On the W-Hair of String Black Holes and the Singularity Problem," TAMU preprint CTP-TAMU-28/92, ACT-7/92 and CERN preprint CERN-TH-6476/92; D. V. Nanopoulos, J. Ellis, and N. Mavromatos, "The Origin of Space-Time as W-Symmetry Breaking in String Theory," Phys. Lett. B288, 23 (1992); D. V. Nanopoulos, J. Ellis, and N. Mavromatos, "World Sheet Duality, Space-Time Foam, and the Quantum Fate of a Stringy Black Hole," Phys. Lett. B289, 25 (1992).

[23] D. V. Nanopoulos, J. E11is, and N. Mavromatos, "String Theory Modifies Quantum Mechanics," Phys. Lett. B293, 37 (1992).

[24] D. V. Nanopoulos, J. Ellis, and N. Mavromatos, "Testing Quantum Mechanics in the Neutral Kaon System," Phys. Lett. B293, 142 (1992).

[25] D. Nanopoulos, J. Ellis, and S. Mohanty, "Quantum Gravity and the Collapse of the Wave Function," Phys. Lett. 221B, 113 (1989) and D. Nanopoulos, J. Ellis, and S. Mohanty, "Wormholes Violate Quantum Mechanics in SQUIDS," Phys. Lett. 235B, 305 (1990). 


\section{Research by C.N. Pope during 1991-1992}

My research over the last twelve months has concentrated primarily on investigating various aspects of string theories based on the extended conformal algebras. Such string theories are known as " $W$ strings."

Ordinary string theory emerges from considering the quantum theory of gravity in two dimensions. Although the metric tensor in two dimensions has no dynamics, it is still the case that it is a gauge field, which means that FadeevPopov ghosts must be introduced when integrating over metrics in the functional integral. These lead to an anomalous term, the central extension, in the Virasoro algebra, which is the symmetry algebra of the gauge-fixed classical action. This anomaly, with central charge -26 , can be cancelled by coupling the theory to conformally-invariant matter with an equal but opposite central charge. The simplest choice for the matter is to take 26 free scalar fields. This gives the starting point for the construction of the 26-dimensional bosonic string.

A similar programme can be carried through for an extended conformal algebra. The first example of an extended conformal algebra was the $W_{3}$ algebra of Zamolodchikov [1]. In addition to the usual energy-momentum tensor $T(z)$, which is the (spin-2) generator of the Virasoro algebra, there is a spin-3 current $W(z)$ as well. These currents do not generate an ordinary Lie algebra, because the terms on the right-hand side of the operator-product expansion (OPE) of $W$ with $W$ are of conformal-spin 4 . These terms can be re-interpreted as a bilinear composite : $T T$ :, a suitably normal-ordered product of two energy-momentum tensors, and so one ends up with a non-linear algebra. Nevertheless, it does satisfy the Jacobi identities.

Simple two-dimensional matter systems, such as a set of free scalar fields, not only have actions invariant under holomorphic and anti-holomorphic Virasoro symmetries, but they are also invariant under non-linear symmetries that can be thought of as classical limits of $W$ algebras. For example, the action given by $L=-\frac{1}{2} \partial \varphi \bar{\partial} \varphi$ is invariant not only under holomorphic reparametrisations $\delta \varphi=$ $k(z) \partial \varphi$, but also under the non-linear transformation $\delta \varphi=\lambda(z)(\partial \varphi)^{2}$. This nonlinear transformation is generated classically by the spin-3 current $W=\frac{1}{3}(\partial \varphi)^{3}$.

To build a $W$-string theory, one starts by gauging the above-mentioned symmetries of the two-dimensional theory. This involves the introduction of a gauge field for each current in the algebra. For $W_{3}$, therefore, one has two gauge fields in the chiral theory, one being the usual $g_{\bar{z} \bar{z}}$ component $h$ of the gauge-fixed metric tensor, and the other is a spin-3 field $B$. The gauging is achieved by adding Noether-couplings $h T+B W$ to the free Lagrangian. In work that I carried out last 
year, with L.J. Romans and K.S. Stelle, we constructed the anomaly-free theory of $W_{3}$ gravity in detail $[2,3]$. One could in principle do this by starting from the classical Lagrangian, with ghosts and gauge fixing terms, and then build up the quantum theory by introducing counterterms and renormalisations of the transformation laws order-by-order in Feynman loops. This is a very tedious process, and instead, in [2,3], we approached the problem by starting from the BRST operator, and following standard procedures to obtain the quantum theory. In practice, what this amounts to is that the original classical currents must be renormalised to give currents that generate a closed algebra-the $W$ algebra-at the full quantum level. Correspondingly, the original classical gauge-transformation rules get renormalised to those generated by the quantum currents. All that remains then is to ensure that the matter realisation of the $W$ algebra gives the appropriate central charge that cancels the contributions from the ghosts associated with the fixing of all the gauge symmetries. The ghosts for a gauge field of spin $s$ contribute $-12 s^{2}+12 s-2$ to the central charge. Thus, in the case of $W_{3}$ gravity, we require a matter system that realises the $W_{3}$ algebra with central charge $c=26+74=100$.

To obtain a $W$-string theory one needs a matter realisation of the $W$ algebra, with the appropriate critical central charge, involving a set of scalar fields that can become the "coordinates" of the target spacetime. The basic known realisations of the $W$ algebras are those that follow from the quantum Miura transformation [4]. For $W_{3}$, this gives a realisation in terms of 2 scalar fields. This is not enough for a physical $W_{3}$ string theory. Fortunately, it was found [5] that the 2-scalar realisation could be extended to a multi-scalar one in a rather straightforward manner. The key point is that one of the two scalars in the Miura realisation enters both $T$ and $W$ only via its energy-momentum tensor, and so one can replace all occurrences of this scalar by an "effective" energy-momentum tensor $T^{e f f}$ with the same central charge as that of the scalar itself. The scalar carries a background charge, and it turns out that the central charge for $T^{e f f}$ must be $c=25 \frac{1}{2}$ in order to achieve criticality for the $W_{3}$ realisation. This realisation is in terms of the effective energy-momentum tensor $T^{e f f}$ and the other of the original two scalars, say $\varphi$.

About a year ago, I had completed a preliminary study, with L.J. Romans, E. Sezgin, and K.S. Stelle, of the spectrum of physical states of the $W_{3}$ string [6]. We found that if one looks for physical states of the form

$$
|\chi\rangle=\mid \text { phys }\rangle \otimes|g h\rangle,
$$

where |phys $\rangle$ is a "tachyon" state with excitations of the matter fields, and $|\mathrm{gh}\rangle$ is the ghost vacuum, then they divide into two categories. First of all, there are 
states which have no excitations in the field $\varphi$, but only excitations in the fields in $t^{e f f}$. For all such states, the momentum $\beta$ in the $\varphi$ direction is "frozen" by the physical-state conditions

$$
\begin{aligned}
\left.\left(L_{0}-4\right) \mid \text { phys }\right\rangle & =0, & \left.W_{0} \mid \text { phys }\right\rangle & =0, \\
\left.L_{n} \mid \text { phys }\right\rangle & =0, & \left.W_{n} \mid \text { phys }\right\rangle & =0, \quad n \geq 1,
\end{aligned}
$$

to only three allowed values, namely $\beta=\frac{6}{7} i Q, i Q, \frac{8}{7} i Q$, where $Q$ is the background charge in the $\varphi$ direction. The remaining physical-state conditions then simply imply that in the remaining "spacetime" described by the fields in $T^{e f f}$, one has effective Virasoro string-like conditions, namely

$$
\begin{aligned}
\left(L_{0}^{e f f}-a^{e f f}\right)|\mathrm{phys}\rangle_{e f f} & =0, \\
L_{n}^{e f f}|\mathrm{phys}\rangle_{\text {eff }} & =0, \quad n \geq 1
\end{aligned}
$$

When $\beta=\frac{6}{7} i Q$ or $\frac{8}{7} i Q$, the value of $a^{\text {eff }}$ is 1 , and when $\beta=i Q$, the value of $a^{\text {eff }}$ is $\frac{15}{16}$.

The other category of states occurs when there are excitations also in the $\varphi$ direction. Again, the physical-state conditions imply that the momentum $\beta$ is frozen to specific values. For example, at level 1 there are such states with $\beta=\frac{10}{7} i Q, \frac{11}{7} i Q$. However, such states will always have zero norm, sincc they do not occur in momentum-conjugate pairs that can have non-zero inner products. The condition for satisfying momentum conservation in the $\varphi$ direction, in the presence of the background charge $Q$, is that $p+p^{\prime}=2 i Q$. Thus we see that the effective states of the previous paragraph satisfy this condition, but the level1 states with excitations in the $\varphi$ direction do not. It seems likely that this phenomenon will persist at all excited levels.

The conclusion of the above, which was developed further in [7], is that if one considers only "standard" states of the form (1), then the only non-null physical states in the $W_{3}$ string are described by a pair of effective Virasoro-like string theories, with $T^{e f f}$ having central charge $c=25 \frac{1}{2}$, and intercepts 1 and $\frac{15}{16}$. The first of these, with the "usual" intercept value, will describe the same massless states as in ordinary string theory, whilst the second will have a spectrum of purely massive states.

In subsequent work during the past year, I have worked on a number of developments of the subject. First of all I examined the generalisation of these results to larger extended conformal algebras. The $W_{3}$ algebra is closely related to $S U(3)$, and there is a natural generalisation to the $W_{N}$ algebra, based on $S U(N)[1,4]$. 
It has currents of each spin in the range $2 \leq s \leq N$. In [7], in collaboration with H. Lu, S. Schrans and K.W. Xu, we studied the details of the $W_{N}$ string theories based on these algebras. One potential stumbling block is that in principle one needs to know the BRST operator for the algebra. In particular, this is need in order to determine the details of the physical-state conditions analogous to (2). It is known for $W_{3}[8]$, but not for the higher $W_{N}$ algebras. Fortunately, it is possible to circumvent the necessity of knowing its explicit form if one can arrive at the physical-state conditions by other means. A conjecture for how to do this was made in [9], and in our work in [7] we were able to give much supporting evidence for its validity. We then went on to show that again, one could obtain from the basic "Miura" realisation of $W_{N}$ in terms of $(N-1)$ scalar fields a more realistic realisation by replacing one of the fields by an effective energy-momentum tensor. The remaining $(N-2)$ scalars then acquire the same "frozen" status as the $\varphi$ field of the $W_{3}$ string discussed above. Again, the only physical states of the standard form (1) that have non-zero norm are those with no excitations in these "frozen" directions, and again one is left witl. states described by effective Virasoro-type conditions (3). This time, criticality requires that $T^{e f f}$ have central charge $c=26-c_{\min }$, where $c_{\min }=1-\frac{6}{N(N+1)}$. Rather strikingly, we see a hint of a relation to the unitary Virasoro minimal models. This is reinforced by the fact that we find that the effective intercepts $a^{\text {eff }}$ in (3) have the form $a^{e f f}=1-\Delta_{k, k}$, where $\Delta_{r, s}$ are the conformal weights of the primary fields in the $(N, N+1)$ minimal model.

In subsequent work, I have extended the results described above to other extended conformal algebras. In [10], with H. Lu, S. Schrans and X.J. Wang, we examined the case of $W$ strings built from $W$ algebras based on the $B_{n}$ and $D_{n}$ Lie algebras, and the exceptional algebras $E_{6}, E_{7}$ and $E_{8}$. Again we were able to construct the necessary multi-scalar realisations of the algebras. A similar picture to that described above emerged. In the process we found also some new realisations of $W$ algebras, in [11,12]. Further extensions of the work, in $[13,14]$, involved looking at supersymmetric extended conformal algebras.

More recently, I have begun to study the structure of discrete states in the two-scalar $W_{3}$ string, and also analogous states in mult scalar $W_{3}$ strings. It was realised some time ago that such states arise in the ordinary string, if the target spacetime is two dimensional [15]. Normally, in 26 dimensions, the excited states of the string describe gauge fields that are described by polarisation tensors with only $26-2=24$ dimensional indices; in other words, two out of the 26 dimensions correspond to longitudinal modes of the gauge fields, and only the remaining 24 dimensions correspond to "transverse" states that have non-zero norm. One might think therefore that in two dimensions there would be no physical states at all. Indeed this is true at generic values of on-shell momentum, but it turns out that 
at special values of the on-shell momentum, there a. non-null physical states, known as discrete states. There existence can essentially be traced back to the fact that the usual "physical" gauges of string theory are in fact not quite physical; the two-dimensional discrete states represent the extent to which the gauge is not in fact physical. The discrete states alluded to above are still of the standard type, described by states of the form (1). There are also more general discrete states in the two-scalar string, which are not of the standard type (1) [16]. Firstly, they may arise at non-standard ghost numbers. Secondly, they may involve ghost excitations as well as matter excitations. An example is the $S L(2, C)$ vacuum in string theory, which has ghost number $G=-1$ and lives at level $\ell=1$. It takes the form $|0\rangle=b_{-1}|g h\rangle$. In [16], it was shown that there are two fundamental operators $x$ and $y$, which give rise to a pair of $G=-1$ discrete states at level $\ell=2$, from which all higher-level discrete states can be built by taking powers of $x$ and $y$. The operators $x$ and $y$ were called "ground-ring generators."

In [17], with E. Sezgin, K.S. Stelle and X.J. Wang, we examined the discrete states of the two-scalar $W_{3}$ string. Again, the $S L(2, C)$ vacuum provides an example of such a state, this time at ghost number $G=-3$ and level $\ell=4$. In terms of the $b, c$ ghost system for the spin-2 gauge field, and the $\beta, \gamma$ ghost system for the spin-3 gauge field, it takes the form $|0\rangle=b_{-1} \beta_{-1} \beta_{-2}|\mathrm{gh}\rangle$. The question then arises as to whether there are analogues of the ground-ring generators $x$ and $y$ of the two-scalar string. In [17], we were able to show that indecd such operators exist. In particular, we found two operators at that generate $G=-3$ states at level $\ell=6$. We also found further discrete states at lower levels, that have nonstandard ghost structure. One of the most important aspects of these discoveries is that they indicate that the full physical spectrum of the $W_{3}$ string is much richer than was previously appreciated. It seems that whereas in ordinary string theory it is only the discrete states of the two-dimensional string that are overlooked if one assumes the "standard" form (1) for all physical states, in the $W_{3}$ string one misses much more. It may be that there is no such thing as a "physical gauge" for the $W_{3}$ string, and so previous studies of the spectrum, based on a rather parallel treatment to that for the ordinary string, have not been general enough to reveal all the richness of the physical spectrum. Indeed, in [17], we found that some of the new "non-standard" states arise also in the multi-scalar $W_{3}$ string where the on-shell momenta are continuous. This is quite unlike the situation for the ordinary string. 


\section{Intended Research by C.N. Pope}

Recent investigations of the structure of discrete states in the $W_{3}$ string have revealed that there is probably a much richer structure of physical states than has been found up until now. They also indicate that the $W_{3}$ string exhibits totally new features that are not observed in the ordinary string; in particular, the rôle played by states with non-standard ghost structure in the multi-scalar theory. It may be that it is in this arena that the true subtleties associated with the non-linearity of the $W_{3}$ algebra emerge.

In collaboration with X.J. Wang, I have a project already under way to try to find the complete set of discrete states in the two-scalar $W_{3}$ string. In addition to the two level $\ell=6$ operators described above, we have been looking for additional operators at level $\ell=8$, which, in conjunction with those at level 6 , should provide the complete set of "ground-ring" generators. Having found these, we will then attempt to determine how one may use them to obtain the full spectrum of discrete states.

In collaboration with B.E.W. Nilsson, H. Lu, K.S. Stelle and P.C. West, I am studying further details of the spectrum of states of the $W_{3}$ string, the classification of its null states, and the consequent gauge symmetries of the physical states in the multi-scalar case. It is important to understand these in order to see just what the physical content of the theory is. After completion of this work, we shall move on to the very important issue of the existence of physical states with non-standard ghost structure in the multi-scalar $W_{3}$ string.

It was found when studying the physical spectrum of $W$ strings that it was often instructive to examine the question in the more general context of $W_{N}$ strings rather than just $W_{3}$. It is probable that further insights will emerge from extending the study of the new discrete states to the $W_{N}$ string, and this is another area that I intend to investigate. In particular, it may help to shed light on the curious relationship between $W_{N}$ strings and Virasoro minimal models, whose precise nature is still obscure.

Recently, a new BRST operator for the $W_{3}$ algebra has been found [18], which has been proposed as the basis for a non-critical $W_{3}$ string. The existence of this new BRST operator raises intriguing questions about the nature of the string theory that may be associated with it, and I intend to begin some investigations in this area also. 


\section{REFERENCES}

[1] A.B. Zamolodchikov, Teo. Math. Fiz. 65 (1985) 347.

[2] C.N. Pope, L.J. Romans and K.S. Stelle, Phys. Lett. B268 (1991) 167.

[3] C.N. Pope, L.J. Romans and K.S. Stelle, Phys. Lett. B269 (1991) 287.

[4] V.A. Fateev and A.B. Zamolodchikov, Nucl. Phys. B280 (1987) 644; V.A. Fateev and S. Lukyanov, Int. J. Mod. Phys. A3 (1988) 507.

[5] L.J. Romans, Nucl. Phys. B352 (1991) 829.

[6] C.N. Pope, L.J. Romans, E. Sezgin and K.S. Stelle, Phys. Lett. B274 (1992) 298.

[7] H. Lu, C.N. Pope, S. Schrans and K.W. Xu, The Complete Spectrum of the $W_{N}$ String, preprint, CTP TAMU-5/92, Nucl. Phys. B, to appear.

[8] J. Thierry-Mieg, Phys. Lett. B197 (1987) 368.

[9] S.R. Das, A. Dhar and S.K. Rama, Physical Properties of $W$ Gravities and $W$ Strings, preprint, TIFR/TH/91-11.

[10] H. Lu, C.N. Pope, S. Schrans and X.J. Wang, Nucl. Phys. B379 (1992) 47.

[11] H. Lu, C.N. Pope, S. Schrans and X.J. Wang, Mod. Phys. Lett. A7 (1992) 1835.

[12] H. Lu and C.N. Pope, Phys. Lett. B268 (1992) 63.

[13] H. Lu, C.N. Pope, X.J. Wang and K.W. Xu, Nucl. Phys. B379 (1992) 24.

[14] H. Lu, C.N. Pope, X.J. Wang and K.W. Xu, Aspects of $N=2$ Super-Wn Strings, preprint, CTP TAMU-29/92, to appear in Int. J. Mod. Phys.

[15] A.M. Polyakov, Mod. Phys. Lett. A6 (1991) 635.

[16] E. Witten, Nucl. Phys. B373 (1992) 187.

[17] C.N. Pope, E. Sezgin, K.S. Stelle and X.J. Wang, Discrete States in the $W_{3}$ String, preprint, CTP TAMU-64/92.

[18] M. Bershadsky, W. Lerche, D. Nemeschansky and N.P. Warner, A BRST Operator for non-critical $W$-Strings, preprint CERN-TH.6582/92. 


\section{Research by Heath Pois During 1991-92}

Over the past year, my research has explored several aspects of supersymmetric and supergravity phenomenology: (i) Aspects of EW symmetry breaking in supergravity models, both at zero and finite temperature, (ii) neutralino relic dark matter densities in the supersymmetric standard model (SSM), and (iii) proton decay and dark matter issues in the minimal supersymmetric (SUSY) SU(5) model.

The present status of supergravity can be summarized by stating that there are several realistic, candidate theories which lead to a remarkably consistent, unified picture of low energy physics that is testable. For example, supergravity theories naturally address the heirarchy problem which exists in any unified theory with a very large mass scale, there is satisfactory unification of the gauge couplings which is consistent with experiment, and the necessity of EW symmetry breaking naturally requires a large top quark mass.

Overall, our investigations of (i-iii) have led to constraints on the parameter space and to specific low energy predictions which may be testable at present and future $e^{+} e^{-}, p \bar{p}$ and $p p$ machines. My collaborators and I have explored some of these predictions in great detail over the past year.

\section{Electroweak (EW) Symmetry Breaking.}

With respect to EW symmetry breaking in minimal Supergravity (SUGRA) models, we have completed a thorough study of the low-energy content of both the supersymmetric standard model(SSM) $[1,2]$ and a class of gauge unified string models. In contrast to previous work [3], we explore the complete and bounded parameter space available to these models which arise after numerous experimental (e.g. masses of $m_{\tilde{l}}, m_{h}$ ) and consistency constraints (EW symmetry breaking, neutral LSP) have been applied 2. As a result, from a handful of initial parameters (the soft SUSY-breaking terms $m_{\frac{1}{2}}, \xi_{0}=m_{0} / m_{\frac{1}{2}}, \xi_{A}=A / m_{\frac{1}{2}}, \tan \beta, m_{t}$ ), we determine the full low-energy sparticle spectrum. We obtain the range of sparticle masses that are allowed within the bounded parameter space, and are correlated via the renormalization group (RG) scaling from $M_{G}$ to $M_{Z}$. Our results demonstrate that various higgs and sparticle masses can and should be within reach of the SSC and possibly LEPII, and distinctive production and decay signatures of these models may well be detected.

Our work updates and refines many of the previous predictions by calculating and including corrections arising from the one-loop effective Higgs potential of the theory. It has been recently shown that the one-loop treatment is essential 
in certain cases (e.g. large $m_{t}$, large tri-linear squark mixing terms) if one is to correctly calculate the physical Higgs mass spectrum and the correct ground state of the theory [4]. We have incorporated these effects completely (including the full s/particle spectrum) and consistently, and present what we believe to be 'state of the art' results which should be useful to experimentalists. The work of [2] generalizes the 'no-scale' results $\left(\xi_{0}, \xi_{A}=0\right)$ obtained in [5].

Recently we have explored the EW phase transition in supergravity models by considering symmetry breaking at finite temperature [6]. Recent work in the area of baryogenesis at the weak scale has led to a reappraisal of the EW phase transition such as the order of the phase transition and the critical temperature, $T_{c}[7]$. In order to not wash out baryon number at the weak scale in the SMI, important constraints arise in the Higgs sector which place upper bounds on $m_{H}$. We have extended the standard model analysis to include the MSSM as well as the SSM. Our preliminary results suggest that the MSSM is barely viable, and agrees with other recent work[8]; in a realistic supergravity scenario, we find that the minimal models are not viable: any unified model which contains only two Higgs doublets or does not have B-L violation at the GUT scale is suspect. This result lends support to other unified models which naturally contain B-L violation.

\section{Cosmology/Astroparticle Physics.}

Another attractive feature of supergravity models is that they contain a natural dark matter candidate, the neutralino $\tilde{\chi}^{0}$. We have recently explored new dark matter constraints on the supersymmetric standard model (SSM) arising from the demands of EW symmetry breaking [9]. This work extends earlier results by correlating the high-energy soft SUSY-breaking parameters to low energy parameters that enter into the one-loop Higgs potential. As a result, the Higgs masses as well as the bino/Higgsino composition of the LSP are specifically determined, and impact directly on the relic neutralino density. We find that large $m_{\vec{q}}$ values are overall disfavored, which translates into values of $\xi_{0} \sim 1$. In order to possibly achieve $\Omega_{\chi} \sim 1$, we also find that $m_{\tilde{l}}>100 \mathrm{GeV}$.

\section{Proton Decay.}

In the context of a specific unified model such as the minimal SUSY $S U(5)$ model, we have explored the additional constraints arising from dimension five $(d=5)$ operators which mediate proton decay via the process $p \rightarrow K^{+} \bar{\nu}$, and may provide the most direct and dramatic verification of the unification paradigm. We have combined the constraints of proton decay and cosmology to the minimal 
SUSY $S U(5)$ model using both the one loop approximation, [10] and using twoloop and sparticle threshold corrections [11]. In addition to restricting the allowed parameter space further, we find striking mass correlations that arise from large values of $\xi_{0}>6$. The addition of the cosmological constraint suggests a possible 'no-lose' prospect for LEPII: if the lightest Higgs (h) is not seen for $m_{h}<50 \mathrm{GeV}$, then the $\chi^{ \pm}<100 \mathrm{GeV}$ and may be seen at either LEPII or Fermilab. Our latest results [11] sharpen some of the previous results obtained in [12]. In addition, SuperKamiokande and Gran Sasso should explore a large fraction of the allowed parameter space. We make some simplifying assumptions regarding the superheavy spectrum; depending on the nature of the superheavy spectrum, proton decay may not be seen in the near future, thus the sparticle mass predictions and correlations that we derive become an important contribution towards a complete test of the model.

Some of my immediate future goals include (i) a more detailed phenomenological analysis of sparticle production and decay signatures relevant to LEPII, which will involve a fully general treatment of chargino and neutralino production, (iii) to explore further constraints imposed by EW precision measurements, and (iii) explore realistic supergravity models which naturally contain B-L violation at the GUT scale. 


\section{REFERENCES}

1) S. Kelley, J. L. Lopez, D. V. Nanopoulos, H. Pois, and K. Yuan, "No-Scale Supergravity Confronts Experiment”, Phys. Lett. B 273 (1991) 423

2) S. Kelley, J. L. Lopez, D. V. Nanopoulos, H. Pois and K. Yuan " $A s$ pects of Radiative Electroweak Breaking in Supergravity Models", CERNTH.6498/92, CTP-TAMU-16/92 Submitted to Nuclear Physics B

3) See J. Ellis and F. Zwirner, Nucl. Phys. B 338 (1990) 317 and references therein

4) For example, see H. E. Haber and R. Hempfling, Phys. Rev. Lett. 66 (1991) 1815; J. Ellis, G. Ridolfi, and F. Zwirner, Phys. Lett. B 257 (1991) 83; Y. Okada, M. Yamaguchi, and T. Yanagida, Prog. Theor. Phys. 85 (1991) 1 .

5) S. Kelley, J. L. Lopez, D. V. Nanopoulos, H. Pois, and K. Yuan, "Higgs Bosons Masses in No-Scale Supergravity", Phys. Lett. B 285 (1992) 61

6) D. V. Nanopoulos, H. Pois, work in progress

7) M. Dine, et al. Phys. Rev. D 46 (1992) 550

8) S. Myint, Phys. Lett. B 287 (1992) 325

9) S. Kelley, J. L. Lopez, D. V. Nanopoulos, H. Pois and K. Yuan "New Constraints on Neutralino Dark Matter in the Supersymmetric Standard Model", CERN-TH.6584/92, CTP-TAMU-56/92 Submitted to Phys. Rev. D

10) J. L. Lopez, D. V. Nanopoulos, and H. Pois, "Proton Decay and Cosmology Stronly Constrain the Minimal SU(5) Supergravity Model", CERNTH.6628/92, CTP-TAMU-61/92

11) J. L. Lopez, D. V. Nanopoulos, H. Pois and A. Zichichi, "Proposed Tests for Minimal SU(5) Supergravity at Fermilab, Gran Sasso, SuperKamiokande. LEP” CERN-TH.6716/92, CTP-TAMU-72/92 
12) R. Arnowitt and P. Nath, Phys. Rev. Lett. 69 (1992) 725; Phys. Lett. B 287 (1992) 89; Phys. Lett. B 289 (1992) 368 

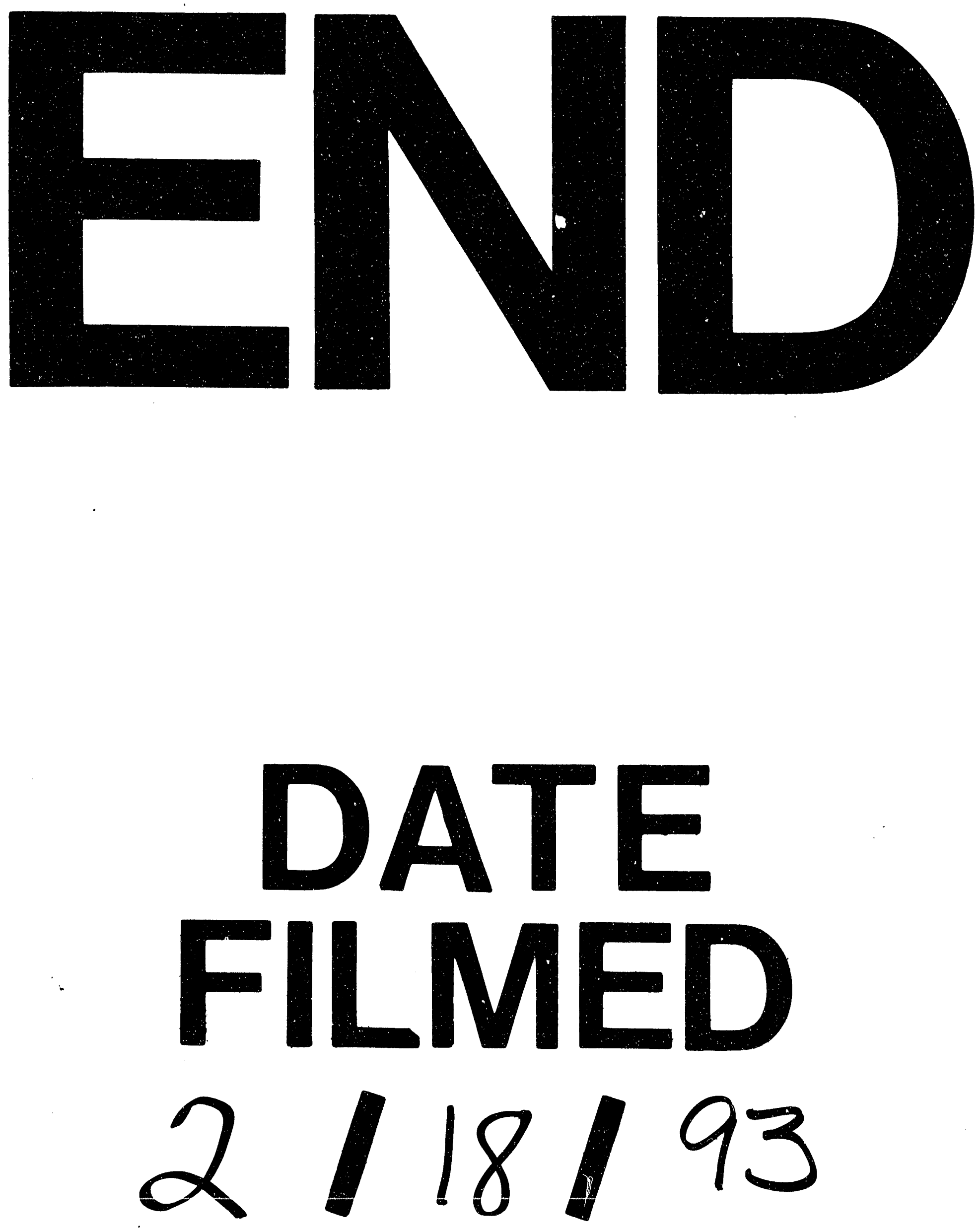
